Zur Homepage der Dissertation

\title{
ORGANIC MOLECULAR NANOTECHNOLOGY
}

\author{
Von der Fakultät für Mathematik und Naturwissenschaften \\ der Carl von Ossietzky Universität Oldenburg \\ zur Erlangung des Grades eines \\ Doktors der Naturwissenschaften (Dr. rer. nat.) \\ angenommene Dissertation
}

vorgelegt von

Dipl.-Chem. Manuela Schiek

geboren am 8. August 1978 in Wilhelmshaven

Tag der Disputation: 27. April 2007 


\section{Hoffnung}

ist nicht die Überzeugung,

dass etwas gut ausgeht, sondern die Gewissheit, dass es Sinn macht, egal wie es ausgeht.

Václav Havel

\section{betreuende Gutachter:}

Prof. Dr. Katharina Al-Shamery (Oldenburg) und Prof. Dr. Arne Lützen (Bonn) weitere Gutachter:

Prof. Dr. Horst-Günter Rubahn (Sonderburg) und Prof. Dr. Ch. Wöll (Bochum)

Die vorliegende Arbeit wurde in der Zeit von Mai 2004 bis Januar 2007 am Institut für Reine und Angewandte Chemie (IRAC) der Carl von Ossietzky Universität Oldenburg und am Institut für Physik und Chemie (IFK) der Süddänischen Universität in Odense durchgeführt.

Hiermit versichere ich, daß ich diese Arbeit selbstständig verfaßt und keine anderen als die angegebenen Quellen und Hilfsmittel benutzt habe.

Farbige Abbildungen online. Publikationen in gedruckter Version. 


\section{Abstract}

Formation of needle-like crystals from inorganic as well as from organic semiconductors such as para-phenylenes, $\alpha$-thiophenes and phenyl-thiophene co-oligomers on dielectric surfaces has attracted increasing interest within the last years. The para-hexaphenylene has proven to be a versatile building block for generating long, parallely aligned organic nanofibers by a self-assembly process on a muscovite mica substrate via sublimation in high vacuum. These nanofibers possess outstanding optical properties like strong, polarised blue fluorescence in high quantum yields after unpolarised excitation with UV light, waveguiding and lasing features. Furthermore, they are chemically and thermically stable and can be easily detached from the growth substrate to serve as key elements in next generation optoelectronic and nanophotonic devices. Owing to their typical height and width on a nanometer scale and length up to one millimeter, the fibers bridge the gap between microscopic and macroscopic dimensions. The morphology of the nanofibers (length, height, width, aspect ratio, bending into rings) is susceptable to process parameters and pretreatment of the substrate. But chemically functionalisation of the molecular building block as key step of this thesis widens the scope of possibilities: Nanoaggregates with tailored morphology and optical properties are generated from designed functionalised molecular building blocks. Functional groups have been implemented at the 1,4' ' ' para-positions of $p$-quaterphenylenes using a non-trivial Suzuki cross-coupling strategy. Symmetrically as well as non-symmetrically functionalised oligomers have been achieved in excellent yields and high purity. Additionally, the synthesis of phenyl-thiophene cooligomers and their acceptability for the nanofiber growth on muscovite mica via organic molecule deposition in high vacuum are presented. The fluorescence peak emission frequency of the nanostructures from the substituted $p$-quaterphenylenes shifts within the blue depending on the functionalisation. While dimensions are still influenced by the growth parameters, the overall shape (i.e. cross-section) of the aggregates alters significantly with the functional group. Even new properties like nonlinear optical activity are accessible. Due to intrinsic nonzero hyperpolarisability of push-pull functionalised oligomers, the respective nanofibers act as frequency doublers and emit strong second harmonic light after excitation with near infrared femtosecond laser pulses. Note that this design approach is possible while conserving the concept of highly crystalline nanofibers. Thus, a new route of bottom-up nanotechnology is presented starting from organic molecular synthesis towards generating of tailormade organic nanofibers opening new prospects for future nanotechnology. 


\section{Zusammenfassung}

Die Bildung von nadelförmigen Kristallen aus anorganischen und organischen Halbleitermolekülen wie zum Beispiel para-Phenylenen, $\alpha$-Thiophenen und Phenylthiophen Co-oligomeren fand großes Interesse in den letzten Jahren. Das para-Hexaphenylen hat sich als besonders geeignet gezeigt lange, parallel angeordnete organische Nanofasern durch einen Selbstanordnungsprozess auf einem Muskovit Glimmer Substrat bei Sublimation im Hochvakuum auszubilden. Diese Nanofasern zeigen außergewöhnliche optische Eigenschaften: Sie senden intensives, polarisiertes blaues Fluoreszenzlicht nach Anregung mit unpolarisiertem UV-Licht aus, außerdem eignen sie sich als Lichtleiter und als Nanolaser. Des weiteren sind sie chemisch und thermisch stabil und können einfach vom Substrat abgelöst werden, um in optoelektronische und nanophotonische Bauteile einer neuen Generation integriert zu werden. Wegen ihrer nanoskalierten Höhe und Breite, und ihrer Länge bis zu einem Millimeter verbinden die Fasern mikroskopische und makroskopische Einheiten miteinander. Die Morphologie der Nanofasern (Länge, Höhe, Breite, Seitenverhältnis, Verbiegen zu Ringen) wird durch die Aufwachsparameter und Vorbehandlung der Substratoberfläche beeinflußt. Eine chemische Modifikation der molekularen Bausteine als Schlüsselelement dieser Arbeit jedoch schafft weitere Möglichkeiten: Nanoaggregate mit bedarfsgerechter Gestalt und abgestimmten optischen Eigenschaften können aus maßgeschneidert funktionalisierten Molekülen generiert werden. Funktionelle Gruppen wurden über eine ausgeklügelte Suzuki Kreuzkupplungsstrategie in den 1,4' ' -para-Positionen von p-Quaterphenylenen eingeführt. Es wurden sowohl symmetrisch als auch nicht symmetrisch substituierte Oligomere in hervorragenden Aubeuten und hoher Reinheit synthetisiert. Außerdem wurden Phenylthiophen Co-oligomere hergestellt, die ebenfalls auf ihre Eignung zur Ausbildung von Nanofasern auf Muskovit Glimmer Oberflächen durch Abscheidung aus der Gasphase im Hochvakuum untersucht wurden. Das Fluoreszenz-Maximum der Nanostrukturen aus den funktionalisierten Quaterphenylenen verschiebt sich innerhalb des blauen Wellenlängenbereichs in Abhängigkeit von der funktionellen Gruppe. Die Abmessungen der Nanofasern werden nach wie vor von den Aufwachsbedingungen beeinflußt, aber die allgemeine Form insbesondere der Querschnitt verändert sich eindeutig bei der Wahl verschiedener Substituenten. Es entstehen sogar neue Eigenschaften wie zum Beispiel nicht-lineare optische Aktivität. Aufgrund der von Null verschiedenen Hyperpolarisierbarkeit, die die nicht-symmetrisch funktionalisierten Molekülbausteine besitzen, sind die jeweiligen Nanofasern Frequenzverdoppler: Sie senden intensives, frequenzverdoppeltes Licht aus, wenn sie mit femtosekunden-gepulstem Laserlicht aus dem nahen Infrarot angeregt werden. Es sei darauf hingewiesen, daß das gesamte Design auf dem Konzept von kristallinen Nanofasern basiert. Dementsprechend wird hier ein neuer Ansatz der sog. bottom-up Nanotechnologie präsentiert, angefangen bei der organischen Synthese bis hin zur Bildung maßgeschneiderter Nanostrukturen, die neue Möglichkeiten für die Nanotechnologie der Zukunft aufzeigen. 


\section{Contents}

\begin{tabular}{lll}
\hline & Introduction to Nanofibers & 7
\end{tabular}

2 Para-Hexaphenylene on Muscovite Mica - a unique combination? $\quad 11$

2.1 Growth Substrate: Muscovite Mica . . . . . . . . . . . . . . . . . 13

3 Synthetic Approach towards functionalised para-Quaterphenylenes [1, 2] 17

4 Growth - Variety of Nanoaggregates 23

4.1 General Remarks . . . . . . . . . . . . . . . . . . . . . . . . . . . . 23

4.2 Symmetrically functionalised para-Quaterphenylenes . . . . . . . . . . . . 27

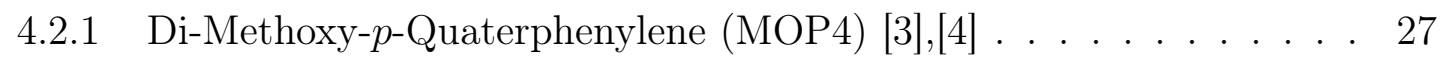

4.2 .2 Di-Chloro- $p$-Quaterphenylene (CLP4) [5, 6$] \ldots \ldots \ldots \ldots$

4.2 .3 Di-Cyano- $p$-Quaterphenylene (CNP4) . . . . . . . . . . . . . . . 37

$4.2 .4 \quad$ Di- $N, N$-Dimethyl-Amino- $p$-Quaterphenylene $(\mathrm{NMeP} 4)$. . . . . . 38

4.2 .5 Di-Amino- $p$-Quaterphenylene (NHP4) . . . . . . . . . . . . . 41

4.2 .6 Di-Nitro- $p$-Quaterphenylene (NOP4) . . . . . . . . . . . . . . . . . . . . . . . . . . . . . . 45

4.3 Mono functionalised para-Quaterphenylenes . . . . . . . . . . . . . . . 45

$4.3 .1 \quad$ 1-Methoxy-p-Quaterphenylene (MOHP4) . . . . . . . . . . . 45

4.3 .2 1-Chloro- $p$-Quaterphenylene (CLHP4) . . . . . . . . . . . . . 46

$4.3 .3 \quad 1-N, N$-Dimethyl-Amino- $p$-Quaterphenylene (NMeHP4) . . . . . . . 48

4.3 .4 1-Cyano- $p$-Quaterphenylene (CNHP4) . . . . . . . . . . . . . 50

4.4 Differently functionalised para-Quaterphenylenes . . . . . . . . . . . . . . . 54

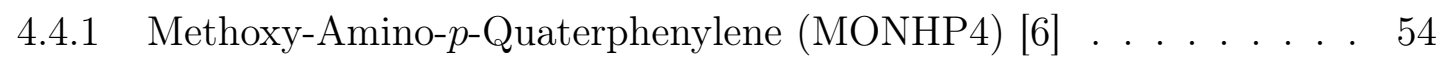

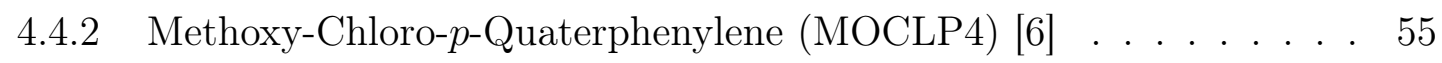

$4.4 .3 \quad$ Methoxy-Cyano- $p$-Quaterphenylene (MOCNP4) . . . . . . . . . . 57

4.5 Nanoshaping . . . . . . . . . . . . . . . . . . . . . . 60

5 Linear Optics 63

5.1 Fluorescence Spectra . . . . . . . . . . . . . . . . . . . . 63

5.1 .1 Symmetrically functionalised $p$-Quaterphenylenes . . . . . . . . . . 65

5.1 .2 Mono functionalised $p$-Quaterphenylenes . . . . . . . . . . . . 68

5.1 .3 Differently functionalised $p$-Quaterphenylenes . . . . . . . . . . . . 72

$5.2 \mathrm{UV} /$ vis Absorption Spectra $\ldots \ldots \ldots \ldots \ldots . \ldots \ldots$ 


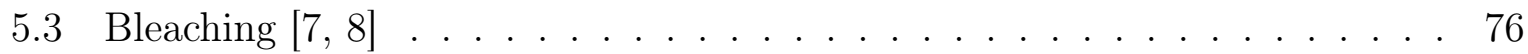

6 Non-linear Optics [6, 9] 79

\begin{tabular}{lll}
\hline 7 & Experimental & 87
\end{tabular}

7.1 Organic Molecular Beam Epitaxy (OMBE) . . . . . . . . . . . . . 87

7.2 Epifluorescence and Darkfield Microscopy . . . . . . . . . . . . . . . . . . . 88

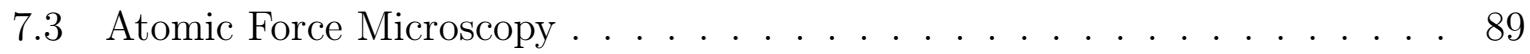

7.4 Measurement of optical properties . . . . . . . . . . . . . . . . . . . . . 91

7.5 Further Characterisation Methods . . . . . . . . . . . . . . . . . . . . . . . 92

7.6 Organic Synthesis . . . . . . . . . . . . . . . . . . . . . . . . . . . . 94

7.6 .1 General remarks . . . . . . . . . . . . . . . . . . . 94

7.6 .2 Symmetrically functionalised para-Quaterphenylenes . . . . . . . . 94

7.6 .3 Non-symmetrically functionalised para-Quaterphenylenes . . . . . . 99

7.6.3.1 $\quad$ Precursors: phenyl building blocks . . . . . . . . . . . . . 99

7.6.3.2 $\quad$ Precursors: biphenyl building blocks . . . . . . . . . . . . 101

7.6.3.3 Precursors: terphenylene building blocks . . . . . . . . . . 107

7.6.4 Symmetrically functionalised para-Hexaphenylenes . . . . . . . . . 112

7.6 .5 Phenyl-thiophene co-oligomers . . . . . . . . . . . . . . . . . . . . . 114

7.6.6 Symmetrically functionalised Phenyl-ethinylene co-oligomers . . . . 115

8 Conclusions and Outlook 119

9 Outlook: Phenyl-thiophene co-oligomers [10] 125

9.1 Synthetic Approach . . . . . . . . . . . . . . . . . . . . . 126

9.2 Growth . . . . . . . . . . . . . . . . . . . . . . . 127

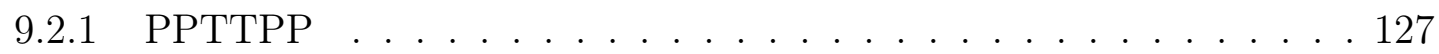

9.2 .2 TTPPTT . . . . . . . . . . . . . . . . . . . 130

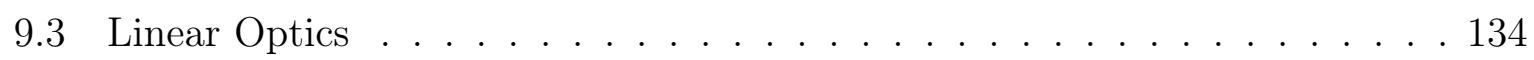

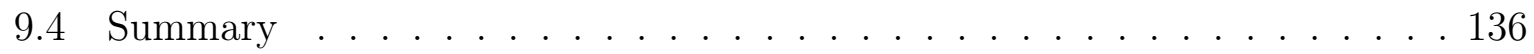

$\begin{array}{ll}10 \text { Acknowledgements } & 161\end{array}$

$\begin{array}{ll}11 \text { Curriculum Vitae } & 163\end{array}$ 


\section{Introduction to Nanofibers}

Bottom-up nanoengineering has been the desire of scientists and engineers ever since the idea of controlling materials down to the nanoscale came to life. Essentially it is the art of fabricating quantitative amounts of similar nanoshaped aggregates from designed molecules with specific properties. Whereas molecular engineering is a long lasting success story, the possibility to nanoshape aggregates via controlled bottom up growth has emerged only within the last ten years. Nanofibers as one modification of nanoaggregates have attracted quite some attention in the recent past, mainly in their form as inorganic semiconducting nanowires [11-15] such as silica nanowires [16-18] for light guiding, nanowire sensors [19, 20] and nanolasers [21], or organic polymer based nanofibers [22 24]. As an example for the latter ones, polyethylene oxide nanofibers produced by electrospinning are shown in Fig.1.1. Semiconducting nanowires promise to revolutionise the field of submicron integrated photonics due to their small sizes and extraordinary morphological and optoelectronic properties.

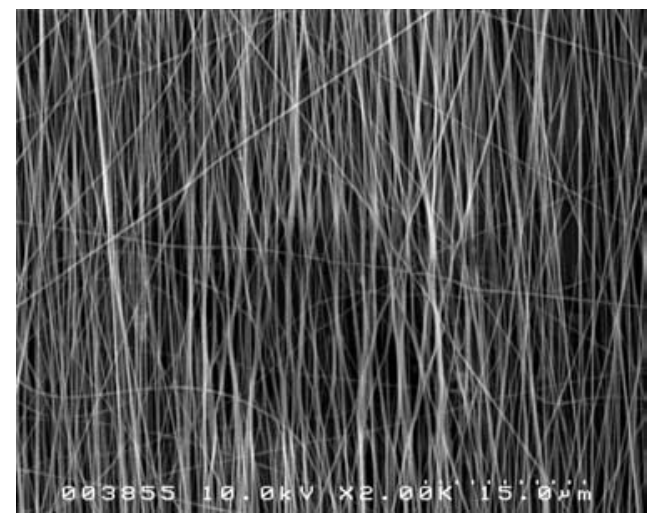

Fig. 1.1: SEM image of polyethylene oxide nanofibers made by electrospinning [25].

A more direct molecular nanotechnology would be to start with organic molecules as building blocks, whose oligomeric properties are easier to tune and to control compared to polymers. The obvious obstacles here are the controlled growth of similar nanoaggregates of predefined shapes and their transfer onto more complicated target substrates. The huge advantage, however, is a tremendously increased design flexibility, enhanced integration into devices and potentially considerably improved performance - comparable to the revolution from liquid crystal based flat screens to organic light emitting device based screens. 

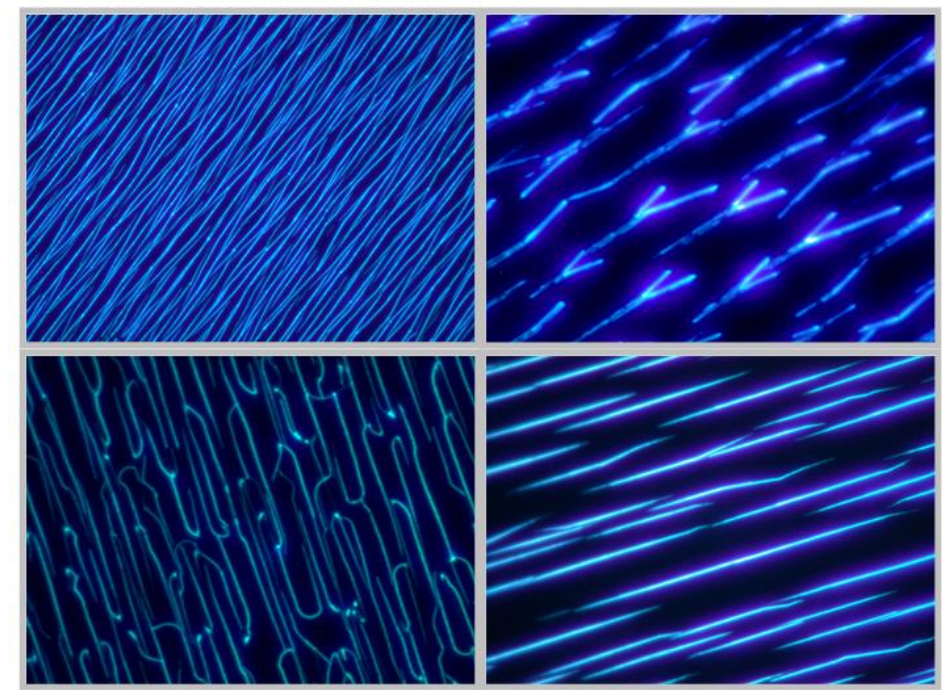

Fig. 1.2: Self-assembly of rodlike functionalised organic oligomers on a muscovite mica substrate upon evaporation in high vacuum leads to well shaped, mutually aligned nanoaggregates. Upon irradiation with UV-light the distinct nature of these aggregates becomes obvious by emission of intense, polarised blue light. The morphology of the nanostructures strongly depends on the functionalisation of the organic oligomers. Not only morphology but also optical properties can be tailored: By choice of an appropriate functionalisation the structures act as nanoscaled frequency doublers. Four fluorescence microscopy images of divers organic nanoaggregates are shown; typical dimensions are several ten nanometer in height, few hundred nanometer in width and length starting from few ten micrometer up to several hundred micrometer. Flexibility is a great advantage of organic molecules compared to inorganic compounds. The developments and exploration of these functionalised organic oligomers is crucial for future nanotechnology.

Among organic molecules oligomers built up of $\pi$-conjugated systems have recently seen a tremendous development due to their interesting optical, electrical and optoelectrical properties [26 30]. The use of organic rather than inorganic compounds to fabricate nanostructures usually has the advantage of higher luminescence efficiency at the same material density, more flexible spectroscopic properties, and in general easier and cheaper processing. Especially rod-like molecules [31, 32] like oligothiophenes [27, 33], perylenes [34, 35], pentacenes [36] and oligo-p-phenylenes [37-40] have been studied in this context [41, 42]. Among those the latter ones and especially the $p$-hexaphenylene has been found to form very interesting needle-like, parallely oriented nanoaggregates upon vapour deposition by a sophisticated high vacuum surface growth process [43, 44]. Owing to their typical height and width on a nanometer scale and length up to one millimeter, the fibers bridge the gap between microscopic and macroscopic dimensions. These nanofibers show high quantum yields being about 30\% [45] of anisotropic blue luminescence, which 
makes them promising candidates for new building blocks for optoelectronic devices like organic light emitting diodes (OLEDs). Waveguides [46 48] as well as ultraviolet light pumped organic nanolasers [49 52] are other potential applications. In addition electrical properties have been investigated [53 55] in view of potential applications of nanofibers in organic field-effect transistors (OFETs) or electroluminescent nanolasers.

Since the para-hexaphenylene has proven to be a versatile building block for generating long, parallely aligned organic nanofibers by self-assembly with outstanding optical properties, an important step in exploiting the full potential of organic nanoaggregates is to functionalise the molecular building block. Such primordial functionalisation is advantageous compared to modifying of existing nanoaggregates. Although the aggregates can be modified by adjusting the growth conditions like substrate temperature, deposition rate or varying and/or modifyfing the growth substrate before deposition of the organic material, this is only possible within rather narrow limits. But chemical functionalisation of the molecular basis widens the scope of possibilities for modifications. This allows not only tailoring and improvment of morphology and optical properties, but also to create new properties. It is challenging to explore prospects and limits of this bottom-up nanoengeneering approach: which chemically functionalised oligomers would still undergo a similar self-assembly process and allow creation of quantitative amounts of crystalline nanofibers with tailored morphologies and optical, electrical, mechanical and even new properties?!

The aim of this thesis is to give an overview and to discover possibilities for future nanotechnology. It is meant as a first step to cross borders and combine organic chemistry, surface science techniques as well as physical characterisation methods. Since the growth of p6P on muscovite mica is already well known and well characterised, it is not topic of this thesis, but used as a starting point. For a better understanding the basic features will be discussed in the following chapter. Chemical functionalisation of the nanofibers' para-phenylene building block is the key step of a promising approach for a new route to designed nanostructures with tailored properties. Note that the aim at this stage is to explore and prove the general feasibility of this approach. Seeing that in-depth interpretations of $\mathrm{p} 6 \mathrm{P}$ nanofibers' properties have been sufficient for several thesises, it would go beyond the scope of a single thesis to apply this to the respective nanostructures of the variety of functionalised para-quaterphenylenes. Thus, a novel organic molecule based bottom-up nanoengeneering concept is presented here, based on the synthesis of functionalised rod-like oligomers, which are expected to develop into an exciting new class of nanomaterials. 
1 Introduction to Nanofibers 


\section{Para-Hexaphenylene on Muscovite Mica - a unique combination?}

Since para-hexaphenylene (p6P) is insoluble in common solvents deposition on substrates has to be carried out by vacuum sublimation processes such as molecular-beam epitaxy [43] or hot-wall epitaxy [44]. Sublimation processes are advantageous compared to precipitation from solution, because the organic molecules deposited from the gas phase in high vacuum with a superior purity suitable for high performance application. In the past p6P has been grown on various solid supports such as dielectric [56 59] and metallic [60 62 surfaces, but muscovite mica emerged as the most favourable growth substrate [39, 63]. The deposition of p6P on a freshly cleaved muscovite mica substrate at elevated substrate temperatures leads to the formation of parallely aligned so called nanofibers, whereas fiberlike aggregates but without parallel alignment are observed on alkali halides [57], titan dioxide [58] and even on phlogophite [10, 63]. According to this muscovite mica turned out to be the ideal match for p6P to generate long, parallely aligned nanofibers.
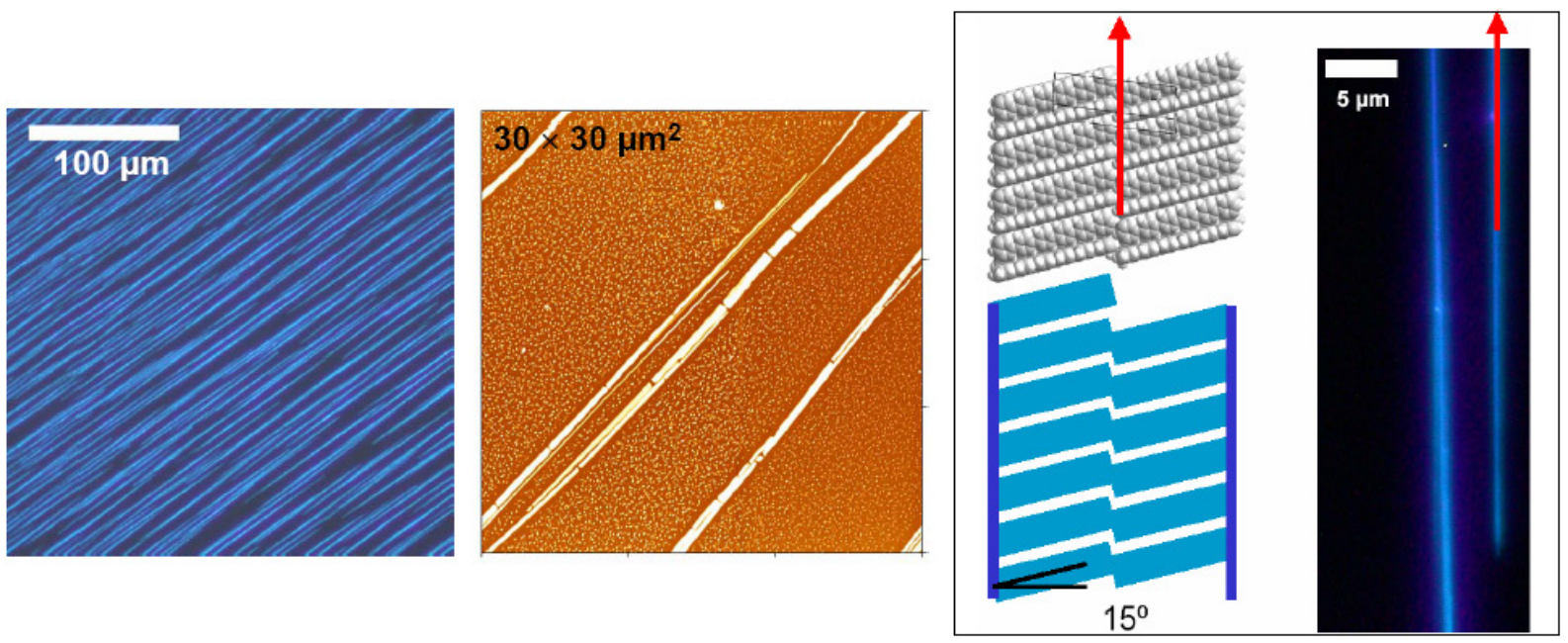

Fig. 2.1: Nanofibers of p6P on muscovite mica: fluorescence microscopy image (left), atomic force microscopy image (middle), and model of molecules' orientation within the p6P nanofibers (right). A p6P oligomer consists of six phenyl rings bonded at the parapositions to give a rod-like molecule.

Heights and widths of the p6P nanofibers are several ten and several hundred nanometer, respectively, while their length can be adjusted between a few ten nanometer and one 


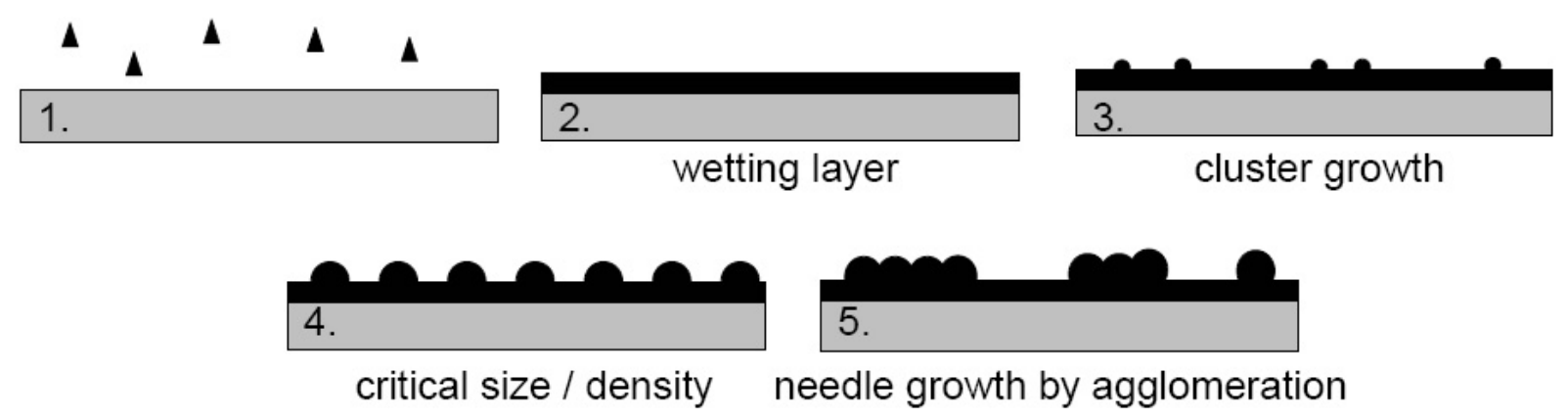

Fig. 2.2: Sketch of growth model for nanofibers via sublimation in high vacuum.

millimeter by varying the growth parameters like substrate temperature, deposition rate and amount of deposited material. In a fluorescence microscope the needles emit polarised blue fluorescence light after unpolarised excitation with UV light, Fig.2.1, owing to their high degree of crystallinity. The plane of polarisation is almost perpendicular to the long fiber axis with an angle of approximately $15^{\circ}$ included. Because of the molecule's symmetry the transition dipole for absorption of UV light and emission of fluorescence light is parallel to the long molecular axis [64]. Therefore, analysis of the nanofiber's polarisation properties gives information about the molecule's orientation within the fibers as shown right hand side in Fig.2.1. This is confirmed by XRD experiments, which reveal that the nanofibers with a herringbone bulk crystal structure are lying with the (1 $\overline{1} \overline{1})$ face parallel to the substrate [65].

Atomic force microscopy (AFM) shows morphological details, Fig.2.1, e.g. width and height, which are not obtainable from optical images. AFM finds additionally small clusters in between the fibers, which are believed to be needle precursors [66, 67] reflecting the bottom-up fashion of the growth process, Fig 2.2. It has been postulated by low energy electron diffraction pattern (LEED) [68] and thermal desorption spectroscopy [69], that a wetting layer of organic molecules is formed on the surface initially. This reduces surface energy, which leads to formation of clusters (i.e. Stransky-Krastanov growth) with the same crystallographic alignment of oligomers as in the wetting layer [65]. The nanofibers grow by agglomeration of these clusters after a critical size / density is reached. They form two different, large domains on a single muscovite mica sample with an angle of $120^{\circ}$ in between. The growth direction is always along muscovite $\langle 110\rangle$. The driving force behind the self-assembled growth is probably a combination of epitaxy and dipole assisted alignment. For a better understanding of the growth process more regard is payed to the growth substrate within the following section. 


\subsection{Growth Substrate: Muscovite Mica}

For generating nanofibers as introduced above an appropriate growth substrate is crucial. Muscovite mica turned out to be the ideal match for p6P as well as for the functionalised para-phenylenes, which will be demonstrated within the following Chapter Growth. In all cases freshly cleaved muscovite mica with the (ideal) formula $\left(\mathrm{K}_{2} \mathrm{Al}_{4}\left[\mathrm{Si}_{6} \mathrm{Al}_{2} \mathrm{O}_{20}\right](\mathrm{OH})_{4}\right)$ was used as growth substrate. It is reasonably cheap and easy to cleave, obtaining a clean and atomically flat surface. This allows preparation of well-defined samples by deposition of organic molecules in high vacuum without too much disturbance from substrate defects like steps as it is the case for e.g. the alkali halides $\mathrm{KCl}$ and $\mathrm{NaCl}$. Muscovite mica is a sheet silicate (monoclinic lattice, $\mathrm{a}=5.2 \AA, \mathrm{b}=9.0 \AA, \mathrm{c}=20.1 \AA, \gamma=95.8^{\circ}$ [70]) consisting of octahedral Al-O layers sandwiched between two tetrahedral Si-O layers. One out of four $\mathrm{Si}$ atoms in the tetrahedral layers is replaced by an $\mathrm{Al}$ atom. The resulting charge due to this substitution is compensated by an intercalation of potassium ions in between two tetrahedral sheets. The cleavage of the substrate occurs along these interlayers and is almost perfect, resulting in large atomically flat areas on the surface. Each cleavage face possesses half of the potassium cations, which leads to the formation of surface dipoles. Their existence has been postulated by low energy electron diffraction pattern (LEED) of vacuum and air-cleaved muscovite mica [71, 72]. A freshly cleaved mica surface is positively charged and hydrophilic.
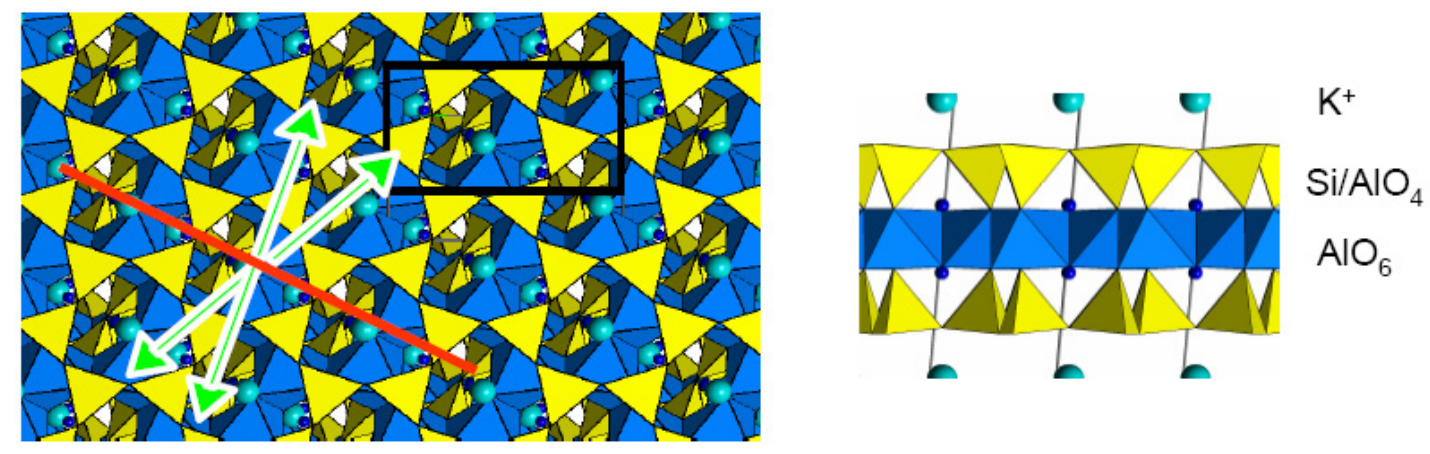

Fig. 2.3: Growth substrate muscovite mica: top view (left hand side), side view along the red line (right hand side). Red line indicates the muscovite $\langle 110\rangle$ direction, which exhibits grooves. Green arrows show surface electric fields, which are connected to the groove direction. Black rectangle indicates the surface unit cell.

Muscovite mica is a dioctahedral mica [73], which means that only two out of three octahedral sites are occupied by a cation. This leads to a tilt of the $\mathrm{Al} / \mathrm{Si}$ oxide tetrahedra and to the formation of grooves along a $\langle 110\rangle$ direction of the surface in case of the most common $2 \mathrm{M}_{1}$ polytype. They have been observed experimentally by atomic force microscopy [74]. These grooves alternate by an angle of $120^{\circ}$ between consecutive cleavage layers together with the oriented electric fields of the surface, leading to grooves along the 
two $\langle 110\rangle$ directions, not along the optical axis [1 000 . That way the surface exhibits a one-fold symmetry instead of a three-fold symmetry, Fig.2.3.

The orientation of the high symmetry directions can be obtained by a so called Schlagfigur. A pin is punched through a mica sample leading to cracks along [1 00$]$, [1 10 ] and

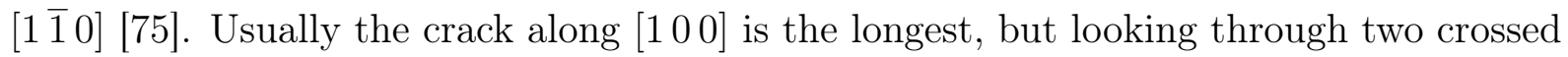
polarisers at the Schlagfigur makes it distinctive: If the light is polarised either parallel or perpendicular to [100] no light passes the polarisers. In case the pin is not sharp enough or pressed with too much force on the mica sample before punching, a Druckfigur is obtained, which is rotated by $30^{\circ}$ with respect to the Schlagfigur [76].

(a)

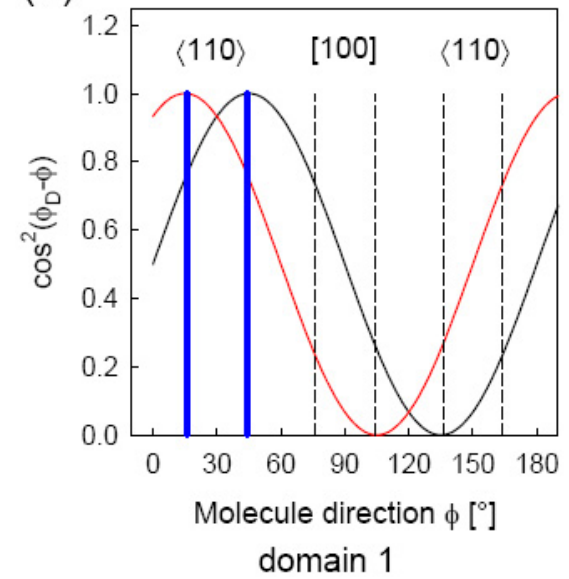

(b)
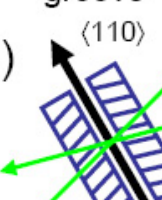

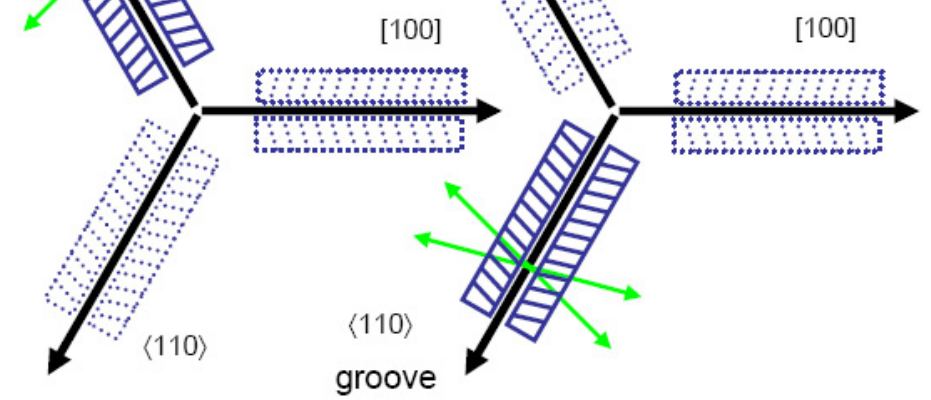

domain 1

Fig. 2.4: Growth model for p6P: (a) For the dipole interaction along $30^{\circ} \pm 15^{\circ}$ the interaction energy $-w(\phi) \propto \cos ^{2}\left(\phi_{D}-\phi\right)$ is plotted. The interaction energies reach their maximum values for needles along $\langle 110\rangle$, i.e. along the two solid vertical lines. In (b) and (c) the orientations of phenylene needles on muscovite mica are sketched, together with the orientation of the molecules within the needles. The muscovite mica directions are depicted by black arrows, the assumed dipole directions by short green arrows. Fibers not realised on the two domains (b) are drawn by dotted lines, in (a) by dashed vertical lines. The calculation in (a) corresponds to the domain in (b). From [63].

The growth direction of the para-phenylene nanofibers is always along a muscovite $\langle 110\rangle$ direction, and this is for symmetry reason the grooved $\langle 110\rangle$ direction. Surface electric fields are assumed to be in along two directions at $90 \pm 15^{\circ}$ with respect to the groove direction. These electric fields are supposed to align molecules on the surface, which was demonstrated many years ago [77, 78. For the nonpolar molecules this alignment is caused by dipole-induced dipole interaction, i.e. a dipole assisted self-assembly [43]. Whereas the long fiber axis is parallel to the high symmetry direction the molecule's long axis is approximately perpendicular to the high symmetry direction with an angle of $\phi=$ 
$\pm 14^{\circ}$. That way the molecules orientate with their long axes parallel to the surface dipoles (angle $\phi_{D}= \pm 15^{\circ}$ ). Because the induced dipole moment depends on the component of the electric field parallel to the long molecular axis, and because the interaction of the induced dipole with the surface dipole depends on the cosine of the enclosed angle, the interaction energy is:

$$
-w(\phi) \propto \cos \left(\phi_{D}-\phi\right)^{2}
$$

The two different surface dipole orientations are just reflected by two different molecule orientations within the fibers (cf. Fig.2.5(c)). In Fig.2.4(a) the azimuthal interaction energy according to Eqn 2.1 is plotted for fixed dipole orientations $\phi_{D}=30 \pm 15^{\circ}$, i.e. dipoles corresponding to the (approximately perpendicular) $\langle 110\rangle$ direction at $\phi=120^{\circ}$. The vertical lines denote molecular orientations matching possible fiber directions. Realised needle directions within a single domain are emphasised by solid vertical lines, not realised directions by dashed lines. Obviously the realised directions are the ones with highest interaction energy. That way on every dipolar domain only a single needle direction exists. This dipolar domain rotates by an angle of $120^{\circ}$ between consecutive cleavage planes. The fibers change their orientation when they cross an odd number of mica cleavage steps, and maintain their orientation for crossing an even number of steps. This results in formation of two domains on a single sample rotated by $120^{\circ}$ with respect to each other.
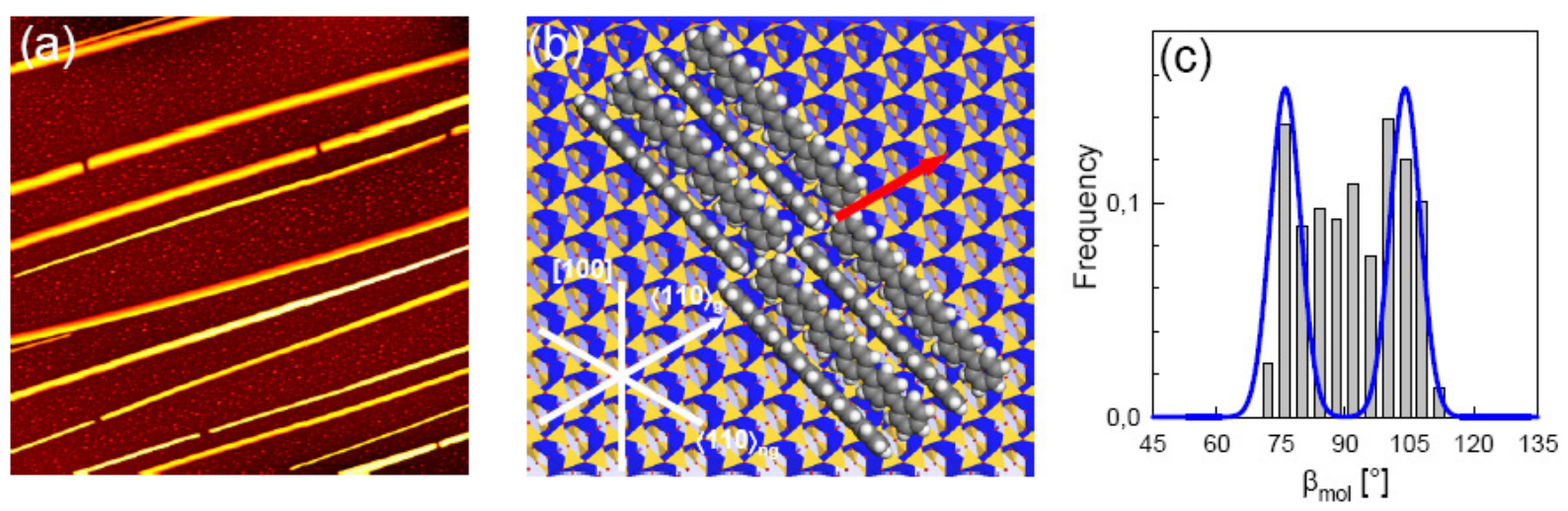

Fig. 2.5: (a) AFM image of $80 \mathrm{~nm}$ tall p6P needles on plain mica, $10 \times 10 \mu \mathrm{m}^{2}$. Needles consist of lying molecules with the $(1 \overline{1} \overline{1})$ face as contact plane, (b) the long needle axis (red arrow) being parallel to the grooved mica $\langle 110\rangle$ direction. In between the nanofibers p6P clusters are visible. In (c) the distribution of the measured angle for maximum polarisation with respect to the long needle axis is shown, together with a solid line representing a model for the optical properties of isolated, single crystalline needles. The deviation between model and measurement for $\beta_{m o l} \approx 90^{\circ}$ stems from, for instance bundled fibers. From [10].

The important role of the grooves and the corresponding electrical fields becomes ob- 
vious, if a mica substrate without these features is used: On phlogopite, a trioctahedral mica without pronounced grooves [73, 74], a single growth direction is no longer preferred, and though needles, namely p6P nanofibers grow along all the three epitaxially favoured high symmetry directions simultaneously [10, 63].

Epitaxy leads to formation of fibers along the high symmetry directions. In general, epitaxy describes the crystallographic relationship between the organic overlayer (the nanofibers) and the growth substrate. The nanofibers face with their close packed (1 $\overline{1} \overline{1})$ face of the herringbone bulk crystal structure the substrate [65], and the unit cell's short axis is oriented along the high symmetry directions [68, i.e. molecules are lying on the surface and their stacking is along muscovite $\langle 110\rangle$, Fig 2.5(b). Since epitaxy alone would result in three growth directions along the high symmetry direction, the dipole induced dipole interactions are responsible for choosing the grooved muscovite $\langle 110\rangle$ directions as the only growth direction. According to this the driving force behind the self-assembled growth process is a combination of epitaxy and dipole assisted alignment.

The question is now: Is this combination of $p$-hexaphenylene as molecular building block and freshly cleaved muscovite mica as growth substrate really unique, or will appropriate primordial functionalisation of the $p$-phenylene molecular basis allow tuning of morphology and of optical properties and even creation of new properties, while conserving the concept of crystalline nanofibers?! 


\section{Synthetic Approach towards functionalised para-Quaterphenylenes [1, 2]}

All previous attempts to realise organic nanofibers from molecules other than $\mathrm{p} 6 \mathrm{P}$ have resulted in only short needle-like structures with no or only weak mutual alignment or more complex needle-like structures [79]. The $p$-phenylene basis gives the highest probability for growth of well-shaped nanofibers on muscovite mica [39]. Comparing the growth of commercially available para-phenylenes consisting of four, five and six phenyl rings, respectively, the nanoaggregates become shorter and less oriented with decreasing chain length of the molecular building block. This is to a certain extend attributed to the decrease in polarisability of the oligomers [43, 68]. So it is tempting to use a $p$-phenylene basis and to maintain approximately the polarisability of the $\mathrm{p} 6 \mathrm{P}$ while changing optical and electronic properties of the molecule via functionalisation. A coplanar arrangement of the molecule's phenylene rings is important for ensuring optimum $\mathrm{CH}$ - $\pi$-interactions 1 between the individual molecules within the crystalline nanoaggregates to maintain the fiberlike structure. Since para-phenylenes are twisted in the gas phase, the surface mobility of the vapour deposited molecules is high enough to migrate on the surface with long diffusion length, which results in promoted epitaxial growth. It has been demonstrated for different phenyl-thiophene co-oligomers with varying degrees of torque in the gas phase, vapour deposited onto $\mathrm{KCl}$, that the rather coplanar molecules favour hit and stick growth, which results in inferior fiber growth [80]. However, the coplanar arrangement within the bulk structure is important for the nanofibers' optical properties because of a better conjugation of the $\pi$-electron systems. Exchange of a hydrogen atom at the meta-position of a phenyl ring by only a small fluorine atom leads to deviation from coplanarity of the phenyl rings within the crystal structure [81, 82. Therefore, the only positions suitable for carrying functional groups seem to be the two para-positions because any other position would lead to a significant out-of-plane orientation of the phenylene rings and probably also prevent the self-assembly for steric reasons.

One could think of two different classes of compounds, symmetrically and non-symmetrically (i.e. differently or mono) substituted oligomers. The latter are more interesting because they offer more possibilities to fine-tune the desired molecular properties and also to exhibit new properties. Due to non-centrosymmetry of the molecules, it is expected from theory that they possess non-zero second order susceptibility [83] 85], therefore the

\footnotetext{
${ }^{1}$ The p6P crystallises in the herringbone packing as typical for many organic molecular crystals.
} 
respective nanoaggregates could feature non-linear optical activity. Molecular engineering has already enabled the design of molecules with specific optical properties such as a large two-photon absorption cross section [86, 87] or large second-harmonic (SH) response [88, 89]. The challenge is now to obtain not only tailor-made oligomers but also morphologically well-defined nanofibers.

Unfortunately, it is rather difficult to modify para-phenylenes because of their low solubility, which even decreases with increasing chain length of the molecule. It is therefore reasonable to try out functionalisation of the rather short para-quaterphenylene oligomers. Since synthesis of the non-symmetrically functionalised oligomers is complex, the symmetrically functionalised oligomers are ideal candidates to prove whether the introduction of functionalities is possible and if the functional groups will be accepted within the growth process.

In the following the development of a general approach for the synthesis of symmetrically and non-symmetrically 1,4' ' -substituted $p$-quaterphenylenes by application of a reliable Suzuki cross-coupling strategy is presented. Experimental details are given as well in the Chapter Experimental. Note that organic synthesis is a key step for the bottom-up nanotechnology approach, therefore it should receive the deserved appreciation.

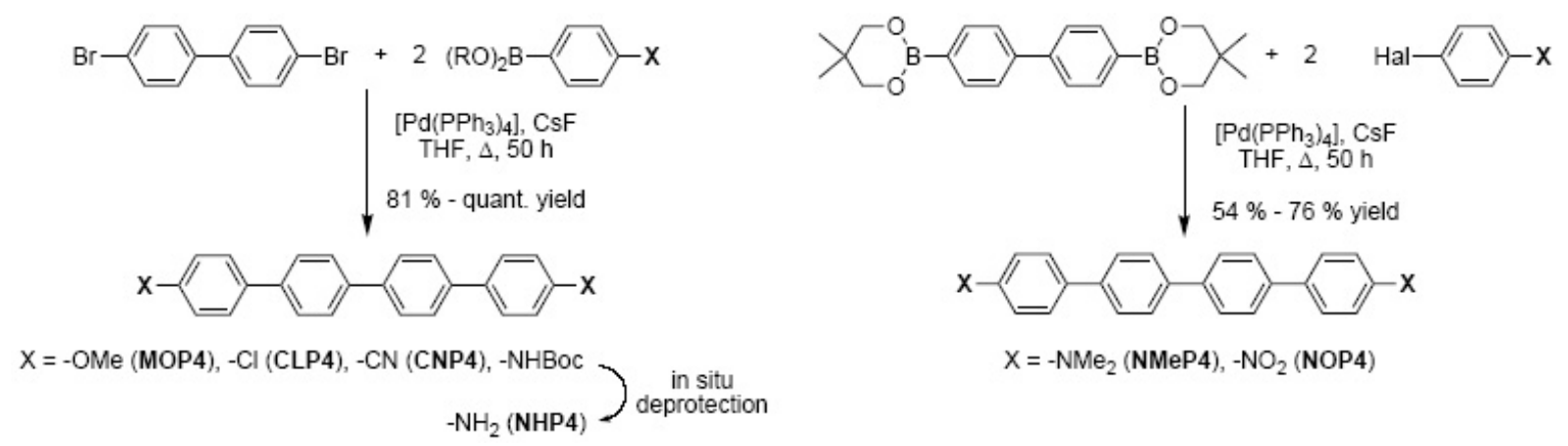

Fig. 3.1: Synthesis of symmetrically $1,4^{\prime \prime}$-disubstituted $p$-quaterphenylenes MOP4, CLP4, CNP4, NHP4, NMeP4 and NOP4 via Suzuki cross-coupling reactions $(\mathrm{R}=\mathrm{H}$ or alkyl).

Although the non-substituted $p$-quaterphenylene has been known for more than 125 years now [90] and like its higher oligomers is even commercially available, 1,4' ''-disubstituted derivatives and especially non-symmetrically functionalised compounds are still rare. This is mainly due to the notoriously low solubility of these compounds that almost prevents a (regio-)selective functionalisation [91, 92]. In order to get access to substances with a defined substitution pattern it is therefore mandatory to introduce the desired functional groups into smaller building blocks and use these precursors to establish the synthesis of the $p$-quaterphenylene scaffold. In the past this was achieved by cyclotrimerisations of acetylenes [93 97], Diels-Alder reactions of cyclopentadienones 
and subsequent aromatisation [98 104, Wittig reactions of cinnamaldehydes followed by Diels-Alder reactions with acetylenic dicarboxylates and subsequent aromatisation [105], addition of Grignard reagents to arines [106 109], Grignard reactions with $p$-quinones and subsequent dehydratisation [110, 111], or Ullmann-type coupling reactions [112].

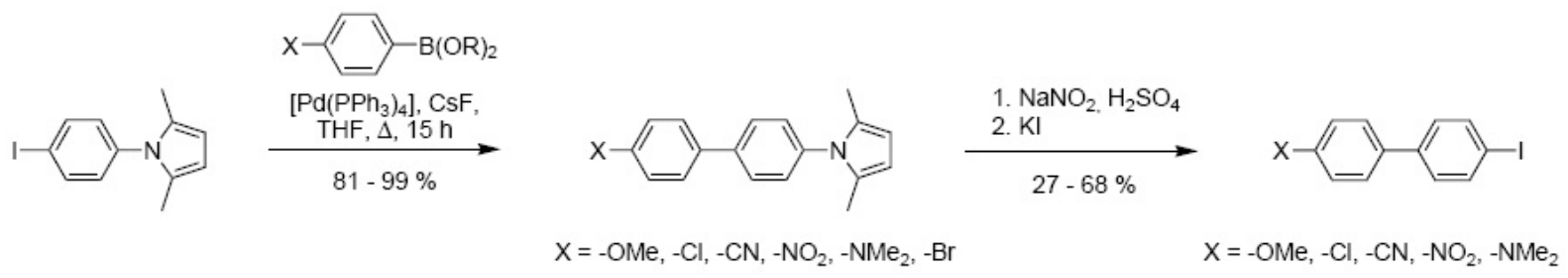

Fig. 3.2: Synthesis of non-symmetrically $1,4^{\prime \prime}$-disubstituted biphenyl building blocks $(\mathrm{R}=\mathrm{H}$ or alkyl).

Modern transition metal catalysed homo- and cross-coupling reactions have become more and more popular over the last 30 years [113 116]. They dominate the synthesis of oligophenylenes today. Especially Kharash- and Suzuki-type couplings using Grignard reagents or arylboronic acids or esters have been used very successfully in this context [117 146]. Although most of the molecules prepared in this way carry long alkyl or alkoxy groups that ensure solubility in common organic solvents a few examples of only 1,4"'disubstituted $p$-quaterphenylenes could also be prepared [118, 119, 137, 147-151].

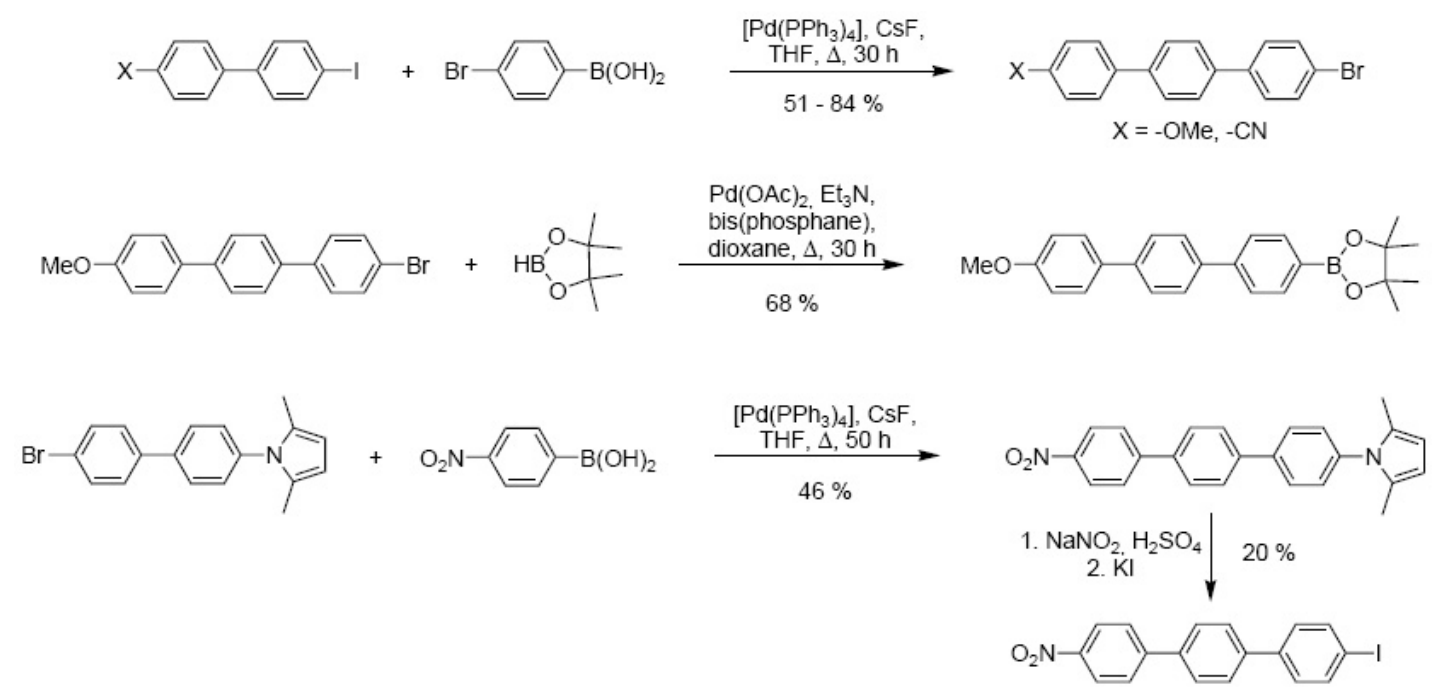

Fig. 3.3: Synthesis of non-symmetrically 1,4"-disubstituted $p$-terphenylene building blocks $(\mathrm{R}=\mathrm{H}$ or alkyl).

Thus, a similar approach for the author's purposes was followed using Suzuki crosscoupling reactions as the key steps in the synthesis of our target compounds.

Symmetrically substituted compounds were synthesised in a two-fold Suzuki crosscoupling reaction from commercially available $p$-substituted phenylboronic acids or esters 
and 4,4'dibromobiphenyl or 4,4'biphenylbisboronic acid ester and a $p$-substituted arylhalide, respectively, using tetrakis(triphenylphosphino)palladium as catalyst (5 mol\%) together with cesium fluoride as base in dry THF as shown in Fig.3.1. The desired products were obtained in 54\% (NOP4), 76\% (NMeP4), 81\% (CNP4), 92\% (CLP4 and NHP4), and quantitative yield (MOP4), respectively, after heating at reflux for $50 \mathrm{~h}$.

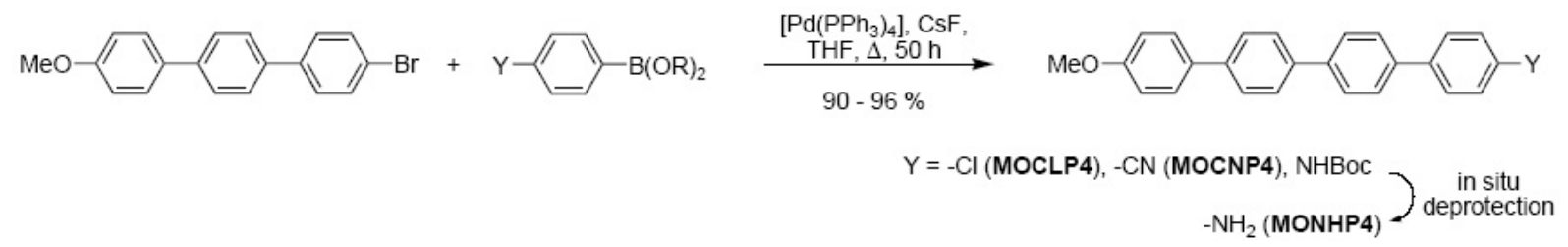

Fig. 3.4: Synthesis of non-symmetrically 1,4"'-disubstituted $p$-quaterphenylenes MOCLP4, MOCNP4 and MONHP4 from a methoxy functionalised $p$-terphenylene $(\mathrm{R}=\mathrm{H}$ or alkyl).

The preparation of non-symmetrically disubstituted derivatives was achieved in a multistep synthesis involving three Suzuki cross-coupling reactions and an iodination reaction as the key steps. It starts with a building block consisting of a single phenyl ring substituted with a protective group and a reactive group in para-positions. Further phenyl rings are added stepwise at reactive groups using Suzuki cross-coupling reactions to finally give the p-quaterphenylene core bearing functional groups at the 1,4' ''-positions (Fig.s 3.2, 3.3. 3.4. The differently functionalised target compounds have been obtained in respectable all over yields around $30 \%$.

This strategy has the advantage of allowing access to a variety of $p$-quaterphenylenes with different combinations of functional groups from the same precursors. Furthermore, it is flexible in the sense that the sequence of Suzuki coupling and iodination reactions can be changed or additional functional group manipulations like palladium catalysed borylations of halogenated compounds can be performed to synthesise other functionalised oligo-p-phenylenes. This is depicted in Fig 3.3 .

Because previous growth studies by the author gave good results for oligomer bearing methoxy groups [3, 4], and differently functionalised oligomers with a methoxy group on one side should be synthetically accessible in good yields, an assortment of those has been synthesised. The three different compounds MOCLP4, MOCNP4 and MONHP4 also carrying a methoxy group at the 1-position and a chloro, cyano or amino substituent in the 4 " '-position, respectively, have been prepared as a first set of non-symmetrically (i.e. differently) substituted $p$-quaterphenylenes in 90\% (MONHP4), 92\% (MOCLP4) and 96\% (MOCNP4) yield (Fig.3.4), respectively.

As in the case of the symmetrical analogues the final products precipitated from the reaction mixture and were washed repeatedly with water and organic solvents for purification. Residual water and organic solvents were then removed by outgassing in vacuo to 
give the desired functionalised compounds in high purity to perform the vapour deposition experiments.

Synthesis of different mono para-functionalised para-quaterphenylenes was carried out by Ivonne Wallmann, University of Bonn [2] by means of Suzuki cross coupling strategy. Four of these oligomers have been chosen for growth studies within this thesis. They are named CLHP4, CNHP4, MOHP4 and NMeHP4 bearing a chloride, cyano, methoxy or $N, N$-dimethylamino group, respectively, at one of the para-positions of the para-quaterphenylene core.

(a)

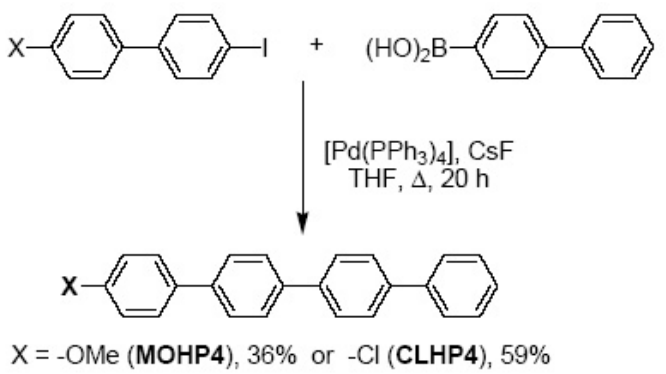

(b)

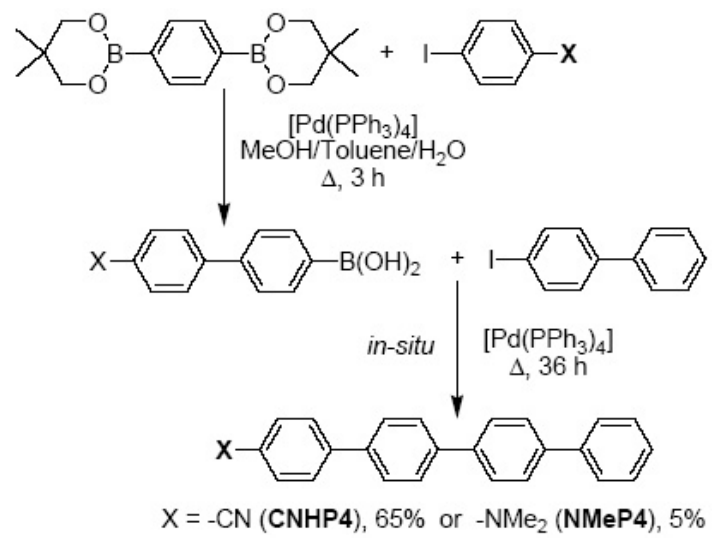

Fig. 3.5: Synthetic approach for the mono functionalised $p$-quaterphenylenes (a) step-bystep reaction for MOHP4 and CLHP4, and (b) one-pot reaction for CNHP4 and NMeHP4.

The preparation of the mono functionalised oligomers was achieved in multi-step reactions using two different approaches with diverse catalytic systems: (I) A step-by-step strategy involving two one-fold Suzuki cross-coupling reactions and a iodination reaction as key steps was applied to synthesise MOHP4 and CLHP4. Yields for the final reaction steps are $36 \%$ and 59\%, respectively, Fig 3.5(a). (II) A one-pot strategy involving two consecutive one-fold Suzuki cross-coupling reactions was utilised to obtain CNHP4 and NMeHP 4 with overall yields of $65 \%$ and 5\%, respectively, Fig. 3.5(b). For purification the poorly soluble products have been washed repeatedly with water and organic solvents. For experimental details see [2].

In conclusion a new practical approach is presented leading to versatile symmetrically, non-symmetrically 1,4' ' -disubstituted and 1-mono substituted $p$-quaterphenylene employing Suzuki cross-coupling reactions. These are promising molecular building blocks for the formation of defined nanoaggregates via vapour deposition techniques. Exploring their acceptability for crystalline nanofiber growth was the next step of this thesis and results are disclosed in the following Chapter Growth.

Additionally, the synthesis of two phenyl-thiophene co-oligomers was carried out by the author. Also their acceptability for the nanofiber growth on muscovite mica via 
organic molecule deposition in high vacuum is demonstrated. Since these co-oligomers are representatives of another interesting class of rod-like organic semiconductors, they are discussed separately in the Chapter Outlook: Phenyl-thiophene co-oligomers. 


\section{Growth - Variety of Nanoaggregates}

\subsection{General Remarks}

So far the most powerful combination for generating long, parallely aligned organic nanofibers consists of para-hexaphenylene as the molecular building block and muscovite mica as the growth substrate. Comparing the growth of commercially available paraphenylenes consisting of four, five and six phenyl rings, respectively, the nanoaggregates become shorter and less oriented with decreasing chain length of the molecular building block. This has been attributed in some degree to a decrease in the static polarisability of the organic molecules along their long molecular axes [63]. The resulting induced dipole moment decreases likewise leading to less stronger dipole induced dipole interactions for the self-assembly process. The symmetrically functionalised $p$-phenylenes are expected from theory to possess a larger polarisabilty compared to the bare $p$-quaterphenylene [83]. For non-symmetrically functionalised $p$-quaterphenylenes of course the permanent dipole moment prevails the induced one. But the value of the polarisability can only give a hint, just useful for comparing the growth of different representatives of a single class of molecules.
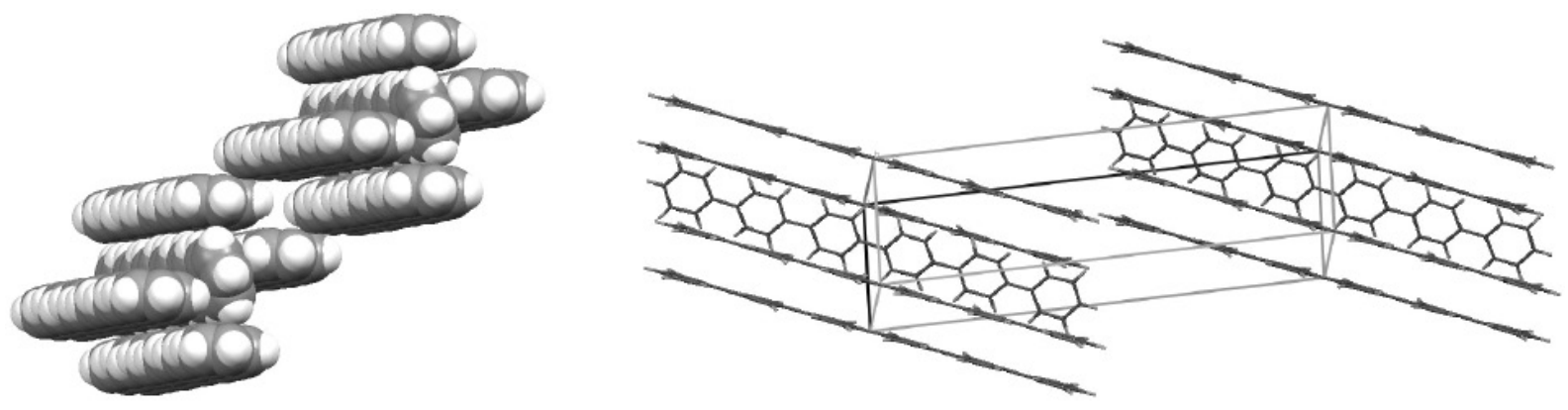

Fig. 4.1: The crystals structure of p6P shows the herringbone packing, which is typical for organic molecular crystals [152]. Solid lines indicate the unit cell.

Another factor which is important for the needle growth is the crystal structure with respect to the substrate, i.e. misfit between organic overlayer and growth substrate, and the internal structure of the unit cell. Therefore, the crystal structure of the molecules is needed for detailed epitaxial considerations. Unfortunately, the crystal structure of the newly synthesised $p$-functionalised $p$-quaterphenylenes is not known yet. The solubility of the para-phenylenes is low, which complicates the growth of single crystals for a structure 


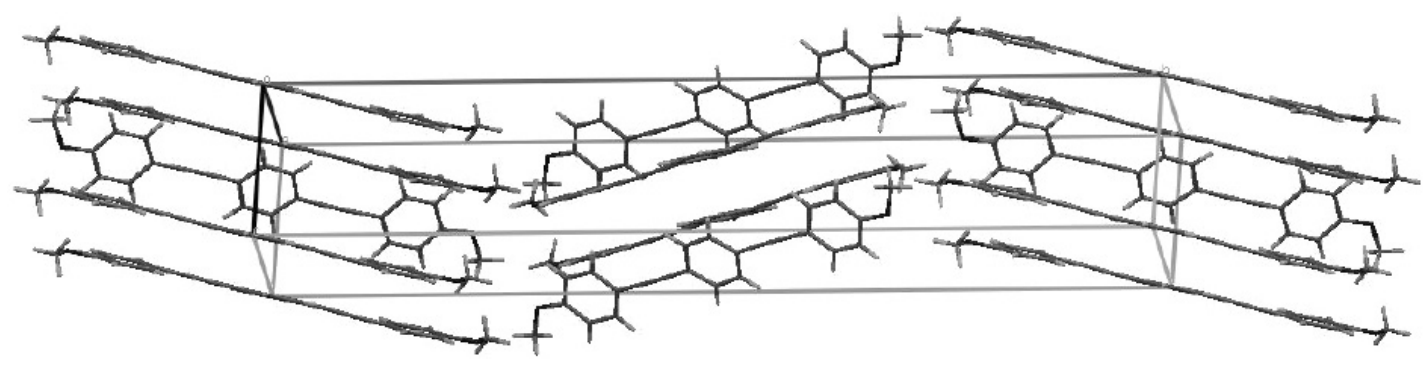

Fig. 4.2: The herringbone crystals structure of MOPE [153]. Solid lines indicate the unit cell.

analysis. The p6P crystallises as herringbone structure, Fig.4.1, which is typical for organic molecular crystals from aromatic molecules, since the herringebone packing allows most dense packing with minimum repulsion [154]. These crystals are held together by per definition weak van-der-Waals interactions, the individual molecule is retained in the crystal. Functionalisation can alter the packing of the molecules considerably. However, for symmetric exchange of the hydrogen in the para-positions with only one different atom, respectively, does not basically change the crystal structure. In case of $4,4{ }^{\prime}{ }^{\prime}$ '-difluoroquaterphenylene (FP4), a symmetric substitution with fluorine atoms at both ends, the typical herringbone structure is retained [155] and the packing does not change. Therefore also the crystal structure for the $p$-quaterphenylene symmetrically functionalised with chloride groups (CLP4) is assumed to be similar to the structure, which has been found for $\mathrm{p} 4 \mathrm{P}$ and $\mathrm{FP} 4$ (Tab.4.1).

\begin{tabular}{cccccccc}
\hline molecule & symmetry & $\mathrm{a}[\AA]$ & $\mathrm{b}[\AA]$ & $\mathrm{c}[\AA]$ & $\beta\left[^{\circ}\right]$ & $Z$ & reference \\
\hline $\mathrm{p} 4 \mathrm{P}$ & $P 2_{1} / a$ & 8.11 & 5.61 & 17.91 & 95.8 & 2 & {$[156]$} \\
$\mathrm{FP} 4$ & $P 2_{1} / a$ & 7.91 & 5.693 & 18.395 & 96.59 & 2 & {$[157$} \\
$\mathrm{p} 6 \mathrm{P}$ & $P 2_{1} / c$ & 26.24 & 5.57 & 8.09 & 98.2 & 2 & {$[156$} \\
$\mathrm{MOPE}$ & $P b c a$ & 6.03 & 40.03 & 7.42 & 90 & 4 & {$[153$} \\
\hline
\end{tabular}

Tab. 4.1: Lattice constants of p4P, FP4, p6P and MOPE crystals.

This might also be the case for bulkier functional groups like methoxy groups at the para-positions of the quaterphenylene basis. In fact the XRD spectrum of MOP4 looks very similar to the spectra of bare phenylenes, with the unit cell slightly enlarged along the moleculas axes [158]. Furthermore, it has been found for phenyl-ethinylene co-oligomers substituted with methoxy groups at both para-positions (MOPE), that these functional groups do not prevent the herringbone structure, Fig.4.2, Tab.4.1. But the packing is different compared to $\mathrm{p} 6 \mathrm{P}, \mathrm{p} 4 \mathrm{P}$ and FP4: In case of MOPE four molecules instead of two belong to a single unit cell, and the molecules are packed in a zig-zag course instead of strictly parallel ordering of the long molecular axes. Note that this changes might 
not be due to the methoxy group but because of the altered molecular backbone, i.e. phenyl-ethinylene instead of para-phenylene backbone. Indeed it has been demonstrated by the author within first trial experiments (cf. [153]), that at this stage the phenyl ethinylenes in general show inferior fiber growth compared to the para-phenylenes. This argues for different packing of phenyl ethinylenes compared to para-phenylenes with a superior match of molecular crystal's and mica substrate's lattice constants for the latter ones. However, the MOPE's crystal structure shows that functional groups in general can be close together, whichs allows intermolecular interactions for appropriate functional groups. The influence of these kinds of interactions on the optical properties in case of mono functionalised para-quaterpheylenes is demonstrated in Chapter Linear Optics.

In the following it will be demonstrated that the deposition of functionalised $p$-quaterphenylenes on muscovite mica via sublimation in high vacuum (for details see Chapter Experimental - OMBE) at elevated substrate temperatures leads in general to fiberlike, parallely orientated nanostructures. Typical deposition rates were $0.1-0.2 \AA /$ s. Since a rather narrow temperature window for generating nanofibers exists [159], the inspected substrate temperatures are ranging from room temperature to about $400 \mathrm{~K}$. The optimum temperatures for fiber growth vary for the individual oligomers, being usually in an individual range of $\pm 15 \mathrm{~K}$ around the respective optimum temperature. In all cases freshly cleaved muscovite mica was used as growth substrate. For generating tailor-made nanofibers not only the design of molecular building blocks is crucial but also an appropriate growth substrate is needed. Luckily muscovite mica turned out to be the ideal match not only for p6P (cf. Chapter A Unique Combination?) but also for functionalised para-phenylenes. Different types of functionalisation have been investigated: (I) symmetrically functionalised oligomers and (II) non-symmetrically functionalised oligomers, namely (II)a) differently di- and (II)b) mono-functionalised oligomers.

The dipole-assisted self-assembly growth process combined with epitaxy (cf. Chapter $A$ Unique Combination?) is not prevented by the functional groups but even improved for some functionalisations. The nanostructures form two domains on a single sample rotated by $120^{\circ}$, and growth is usually along muscovite $\langle 110\rangle$ if not otherwise indicated. The morphology and the optical properties of the resulting nanostructures are determined by the functional groups attached to the molecular building block. Most of the nanostructures show strong, polarised blue luminescence after excitation with unpolarised UV-light under normal incidence, which indicates lying molecules on the surface as light emitters [160, 161 ordered with a high degree of crystallinity. The plane of polarisation is always approximately perpendicular to muscovite $\langle 110\rangle$, i.e. almost perpendicular to the long fiber axes. Because of the assumed herringbone structure with parallely packing of the molecules, and because of the main transition dipole being approximately parallel to the long molecular axis, analysing of the polarised emission gives a clue about the molecules' 
orientations within the nanofibers. These polarisation properties are of course optical properties, but they have to be viewed together with the structure of the respective nanoaggregates, therefore they are presented within this Chapter Growth.

The exact fluorescence wavelength is tunable within the blue by the functional groups attached to the molecular building block to a certain extend (see Chapter Linear Optics). Also non-fluorescent nanofibers can be generated, which is due to fluorescence quenching of some certain functional groups. The detailed morphology, i.e. dimensions, crosssection shape, aggregate density are determinded by the functional groups as well (see this Chapter and Nanoshaping). The non-symmetric functionalisation allows new optical properties: Since the oligomers are expected from theory to possess non-zero second order susceptibility [83 85], the respective nanofibers show non-linear optical activity and act as nanoscaled frequency doublers (see Chapter Non-linear Optics). The noncentrosymmetry of the oligomers must be retained for the bulk structure to allow this optical second harmonic generation. Therefore, a herringbone packing with a head-to-tail orientation of the oligomers with respect to their functional groups is assumed for the non-symmetrically substituted $p$-quaterphenylenes. In case of the mono functionalised oligomers pronounced intermolecular interactions via hydrogen bondings can be deduced from the fluorescence spectra (see Chapter Linear Optics).

In the following sections, apart from a few exemplarily statistical analyses, in general an overview of the individual structural features of the nanoaggregates is given, i.e. rough specifications on features such as dimensions and aggregate number density, aiming at qualitative and comparing impressions, focussing on representative features. 


\subsection{Symmetrically functionalised para-Quaterphenylenes}

\subsubsection{Di-Methoxy-p-Quaterphenylene (MOP4) [3], [4]}

The brightly blue fluorescent nanofibers from MOP4 are almost parallely aligned with a mean width and height of several hundred nanometers and several ten nanometers, respectively, and a length of several hundred micrometers. For the optimum substrate temperature $\mathrm{T}_{S} \approx 340 \mathrm{~K}$, fibers with length up to $800 \mu \mathrm{m}$ are nearly as long as the prominent $\mathrm{p} 6 \mathrm{P}$ fibers. Some fibers possess strongly asymmetric borders. This phenomen is known for p6P fibers aswell, but the asymmetry is is much less pronounced. If a critical width of about $700 \mathrm{~nm}$ is reached for MOP4 fibers, a lateral instability leads to sawtoothlike aggregates with one rugged and one straight side (Fig.4.3(a)). No preference for the rugged sides being either on the left-hand or on the right-hand side of a needle is observed. The fibers are in general wider and flatter compared to p6P fibers for similar growth conditions. The top surface of MOP4 aggregates is exceedingly flat, the crosssection is slablike.
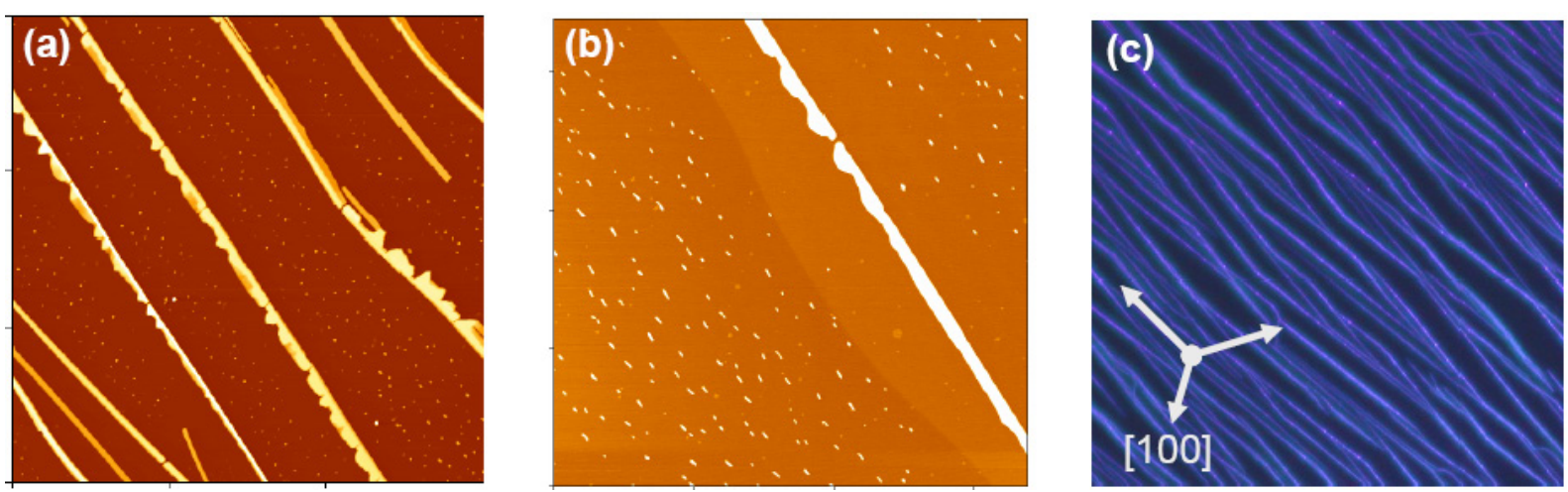

Fig. 4.3: AFM images (a) $30 \times 30 \mu \mathrm{m}^{2}$, height scale $110 \mathrm{~nm}$; (b) $17 \times 17 \mu \mathrm{m}^{2}$, height scale $38 \mathrm{~nm}$ and fluorescence microscope image (c) $250 \times 250 \mu \mathrm{m}^{2}$ of a single domain of MOP4fibers grown on mica at a substrate temperature of $T_{S}=335 \mathrm{~K}$. (a) demonstrates the occurrence of a lateral instability, which occurs if the needles are wider than approximately $700 \mathrm{~nm}$. In (b) a subnanometer step (stepheight $0.8 \mathrm{~nm}$ ) on the surface is clearly visible, attributed to two steps within a wetting layer of lying molecules. The nominal thickness for all images is $4 \mathrm{~nm}$. In (c) the muscovite crystallographic directions [1 000 and $\langle 110\rangle$ are emphasised. From [4].

Small clusters are located in between the needles, which are belived to be needle precursors [66, 67]. Around the fibers a depletion zone of several hundred nanometers up to a few micrometers exists, which is larger than the depletion zone around p6P fibers. This indicates a higher mobility of molecules and clusters on the surface in case of MOP4. The 
effect of surface mobility becomes more evident if one compares the growth for different substrate temperatures. For lower surface temperatures such as $\mathrm{T}_{S}=300 \mathrm{~K}$ the fibers are more densely packed and the mean height decreases to about $40 \mathrm{~nm}$ instead of being around $60 \mathrm{~nm}$ high for $T_{S}=335 \mathrm{~K}$. Also the mean length decreases significantly down to few ten nanometers. The lateral instability for wider needles (width $>700 \mathrm{~nm}$ ) is retained for the lower surface temperatures. Fig 4.4 shows needle growth for an increasing amount of organic material deposited at $\mathrm{T}_{S}=300 \mathrm{~K}$. Initially short needles are formed, followed by a closely-packed film of short fibers.
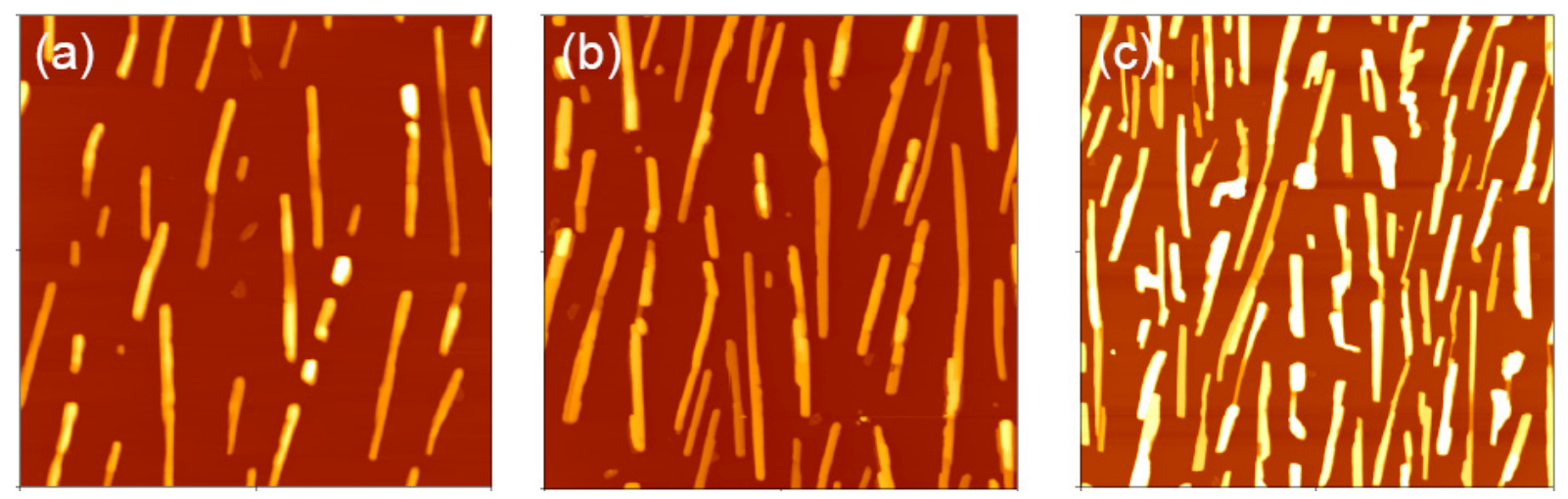

Fig. 4.4: AFM images $\left(10 \times 10 \mu \mathrm{m}^{2}\right.$, height scales $\left.75 \mathrm{~nm}\right)$ of MOP4 grown on muscovite mica at $\mathrm{T}_{S}=300 \mathrm{~K}$. From (a) to (c) the nominal thickness of deposited organic material increases from $1 \mathrm{~nm}$, over $2 \mathrm{~nm}$, to about $6 \mathrm{~nm}$. From [4].

The fibers form two domains on a single mica sample, which are mutually rotated by $120^{\circ}$. The orientation of the needles with respect to the structurally different muscovite high symmetry directions $\left[\begin{array}{ll}1 & 0\end{array}\right]$ and $\langle 110\rangle$ can be obtained with an uncertainty of $\pm 3^{\circ}$ from a Schlagfigur. In this manner it is found out that the mean growth direction of the fibers is always along muscovite $\langle 110\rangle$ and never along [1 00$]$ (Fig.4.3(c)). So again the surface electric fields allow one out of three otherwise equivalent crystallographic orientations to be distinguished from the others in favour of dipole induced dipole aligned growth. This is typically not only for p6P (see previous chapter) and MOP4, but for all other functionalised $p$-quaterphenylenes and will be assumed as a known feature in the following sections. A special feature for MOP4 is that within each domain two orientations with an angle of about $14^{\circ}$ in between are visible: The nanofibers are regularly bent every few ten micrometers to form kinks. They are oriented at $\pm 7^{\circ}$ (experimental error $\pm 3^{\circ}$ ) with respect to muscovite $\langle 110\rangle$. A possible explanation for the two orientations within a single domain considers an epitaxial relationship with the substrate: Needles with the same crystallographic orientation can be mirrored along the high symmetry direction. Or two types of needles exist with different crystal faces being parallel to the substrate.

The dichroic ratio of the polarised light is close to one and even higher than in case of $\mathrm{p} 6 \mathrm{P}$ nanofibers, therfore the fluorescence is used to elucidate the two different needle 

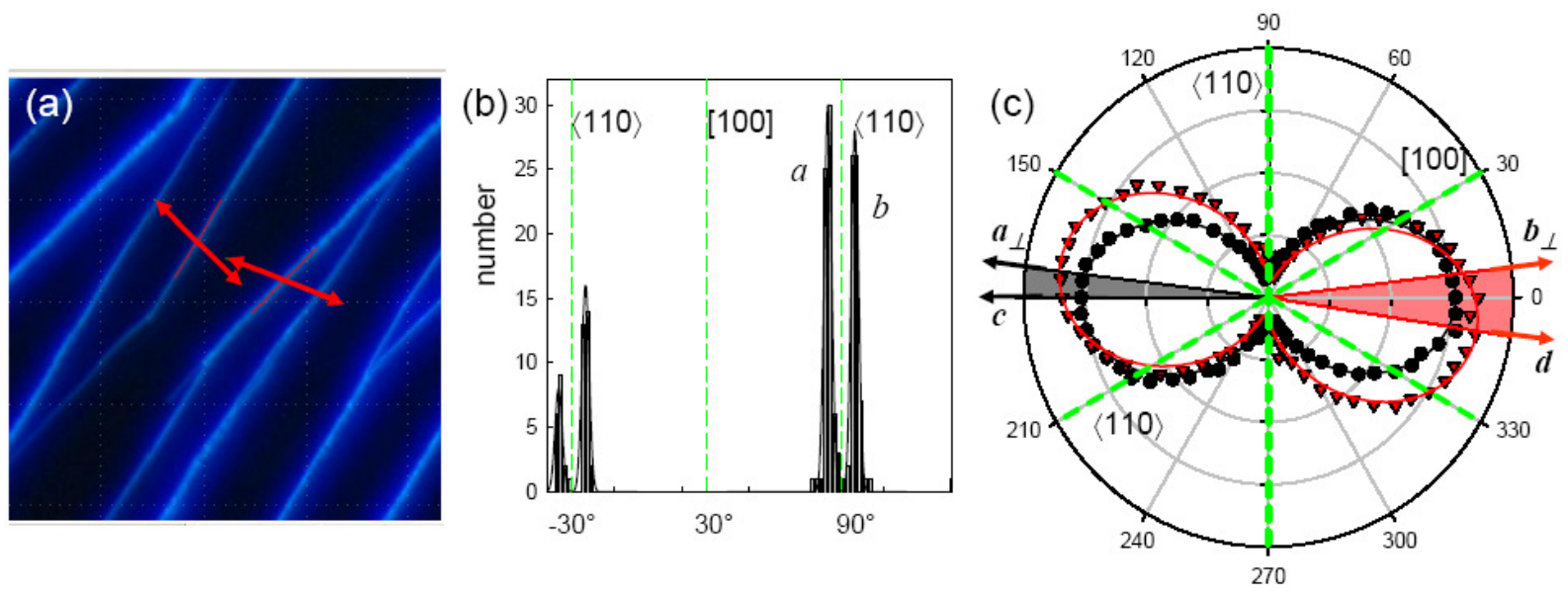

Fig. 4.5: (a) Fluorescence microscopy image $\left(70 \times 70 \mu \mathrm{m}^{2}\right)$ of MOP4 needles shows the two different needle orientations within one domain. The red arrows mark the polarisation direction, i.e. the transition dipoles of the molecules. (b) Angular distribution of MOP4 needles on muscovite mica. The mica crystallographic directions $\left\langle\begin{array}{llll}1 & 1 & 0\end{array}\right\rangle$ and $\left[\begin{array}{lll}1 & 0 & 0\end{array}\right]$ are depicted by dashed vertical lines. (c) For the two needle directions around $90^{\circ}$ the polarised fluorescence intensity after unpolarised UV excitation is plotted. Circles correspond to a needle from distribution $a$, triangles to a needle from distribution $b$; the solid lines are $\cos ^{2}$ fits. Luminescence maxima $c$ and $d$ show an asymmetric offset with respect to the directions perpendicular to the long fiber axes, $a_{\perp}$ and $b_{\perp}$, emphasised by the coloured areas. From [4].

orientations. The molecular arrangement determines the optical properties. The lowest lying transition dipole of the molecules is oriented along their long molecular axis, i.e. blue fluorescence light is polarised along this long molecular axis. The polarisation is almost perpendicular to the long fiber axes. This indicates that the molecules are lying with their long axes almost perpendicular to the long fiber axes. Each fiber direction has its own polarisation direction, i.e. two polarisation directions exist. On the left-hand side of Fig. 4.5 a fluorescence microscopy image of MOP4 fibers is shown and the polarisation directions for needle representatives of the two orientations within one domain are marked by red arrows. On the right-hand side of Fig 4.5 the angular distributions of the fluorescence intensity after unpolarised UV excitation for needles from orientations $a$ and $b$ are depicted by circles and triangles, respectively, together with $\cos ^{2}$ fits representing Malus law (see Chapter Experimental). In the middle of Fig 4.5 the orientational distribution of needles is presented. The two orientations $a$ and $b$ of MOP4 needles on muscovite mica are shown with respect to the high symmetry directions. The dashed lines picture the mica crystallographic directions. The $a_{\perp}$ and $b_{\perp}$ directions are perpendicular to $a$ and $b$, repectively. For orientation $a$ the angle between its fluorescence maximum $c$ and $a_{\perp}$ is $\angle\left(a_{\perp}, c\right)=(8 \pm 4)^{\circ}$, whereas for orientation $b$ the angle between the fluorescence maximum 
$d$ and $b_{\perp}$ is $\angle\left(b_{\perp}, d\right)=(15 \pm 4)^{\circ}$. This means that the polarisation direction of the light for the needle orientation $a$ is along the mica high symmetry direction $\langle 110\rangle$. For the needle direction $b$ the polarisation direction is rotated by an angle of $(8 \pm 4)^{\circ}$ with respect to $\langle 110\rangle$. Thus, although the fiber orientations within a single domain are symmetrically distributed around $\langle 110\rangle$, their optical polarisation properties are slightly different. Due to this asymmetry it can be proposed that the two needle directions reflect two crystallographically different needles either two different contact faces or two different polymorphs.

A wetting layer has been postulated for $\mathrm{p} 6 \mathrm{P}$ on muscovite from LEED patterns [63] as well as from thermal desorption spectroscopy [69]. In case of MOP4 a direct evidence for its existence is shown by means of atomic force microscopy for the first time. In Fig 4.3(b) a $0.8 \mathrm{~nm}$ tall step running almost along the fiber direction is clearly imaged. Steps with a height of $0.4 \mathrm{~nm}$ can be found as well. On the plain mica surface steps are $1 \mathrm{~nm}$ tall. Also subnanometer-steps have been reported, but attributed to potassium cations [162]. These steps vanish within minutes after cleavage.
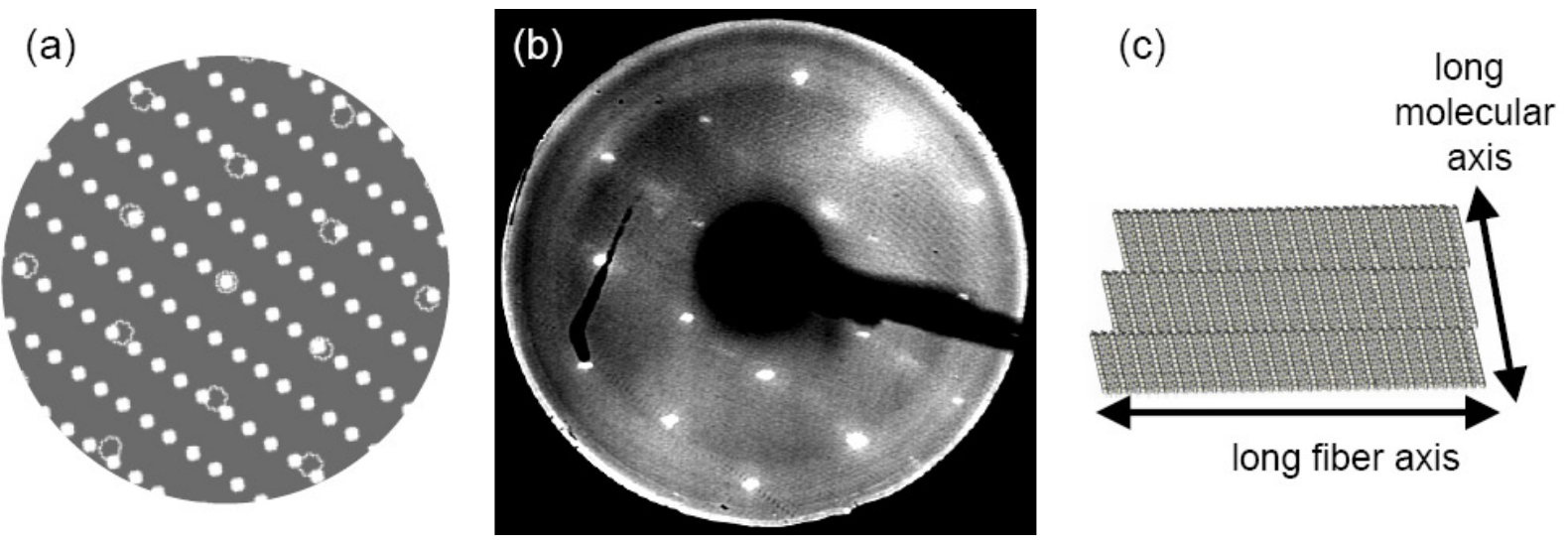

Fig. 4.6: (a) Simulated LEED pattern [163] of muscovite mica (hollow cicles) with organic overlayer (filled circles). (b) LEED pattern (electron energy $E=111 \mathrm{eV}$ ) of MOP4 deposited on muscovite mica at $T_{S}=335 \mathrm{~K}$. Bright spots stem from the muscovite substrate, whereas the weak stripes, running from top left to bottom right through the spots, are due to the organic overlayer. (c) Model for the orientation of molecules within a fiber.

Optical microscopy as well as atomic force microscopy are real space methods for characterisation of nanofiber-samples ex situ under ambient air conditions. Diffraction methods provide additional information about order and symmetry of the sample. Here the diffraction of low energy electrons (LEED) is used to characterise the organic nanoaggregates in situ immediatly after growth. In the LEED diffraction pattern (Fig.4.6(b)) both the hexagonal pattern from the mica substrate as well as a weak superstructure from the organic molecules are visible. This superstructure consists of weak parallel stripes running 
from top left to bottom right through the bright diffration spots from the mica substrate. A simulated LEED pattern of a single domain of muscovite mica with lying p4P molecules as organic overlayer is shown in Fig.4.6(a), which is similar to the measured pattern of MOP4 reproducing the main features. Because almost identical LEED patterns have been described for the case of $\mathrm{p} 4 \mathrm{P}$ on muscovite mica [68], this pattern is attributed to lying molecules on the surface with their long molecular axes approximately perpendicular to the long fiber axes. Only a small fraction of the surface area is covered by nanoaggregates, therefore the observed LEED patterns are accredited to a wetting layer of lying MOP4-molecules on the surface. The lateral orientation of these molecules is similar to the orientation of the molecules within the nanoaggregates, i.e. perpendicular to the needle direction. This is revealed by a careful comparison of the LEED pattern with optical microscope images from the same sample, and it is in accordance with the results from analysing the fiber's polarisation properties as discussed above. The relatively diffuse diffraction features might be related to different molecule orientations within the wetting layer.

\subsubsection{Di-Chloro-p-Quaterphenylene (CLP4) [5, 6]}

Vapour deposition of CLP4 on muscovite mica leads to formation of mutually aligned nanostructures. In a fluorescence microscope they emit polarised blue light after excitation with UV light from a high-pressure mercury lamp (Fig 4.7).
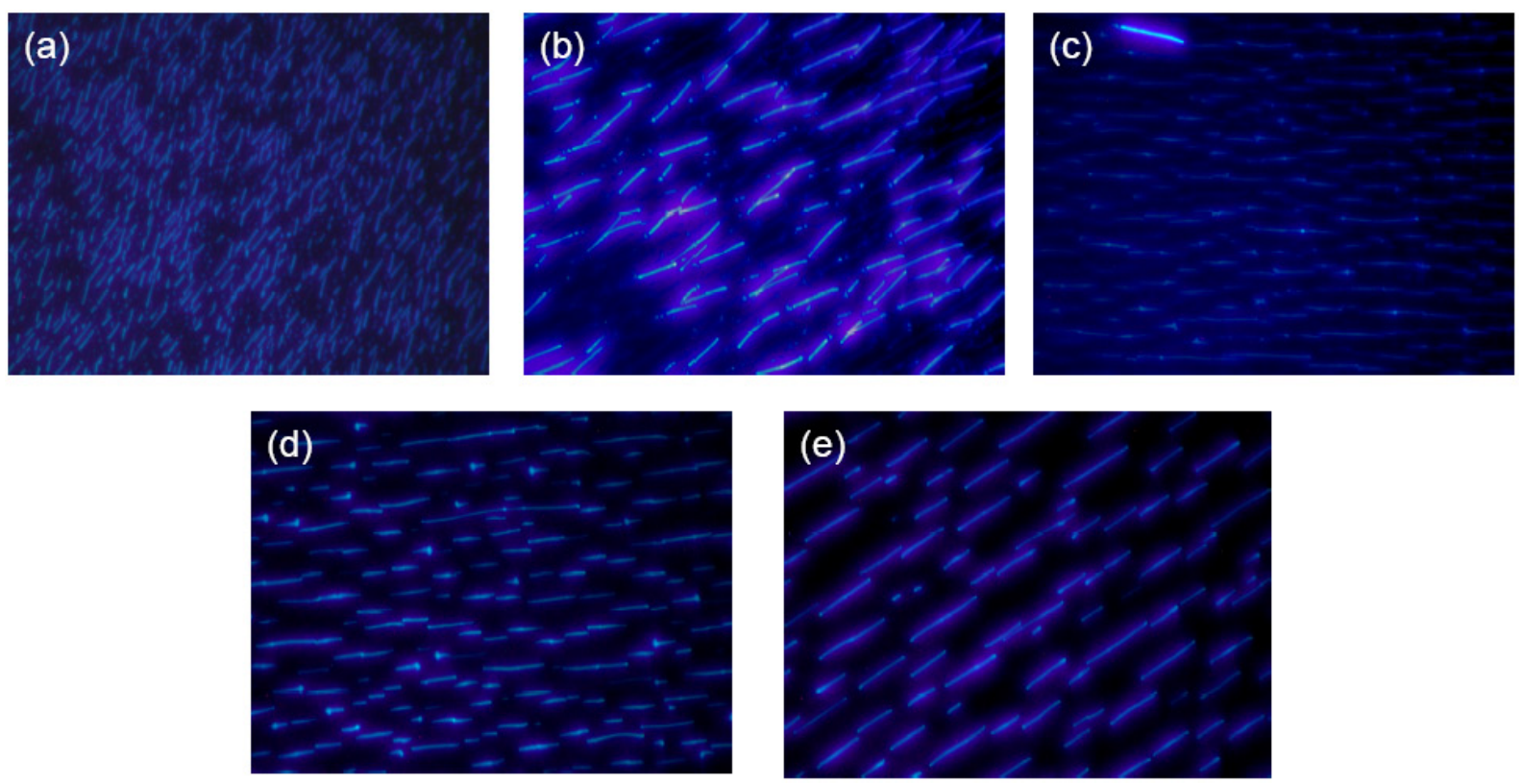

Fig. 4.7: Fluorescence microscopy images $\left(85 \times 113 \mu \mathrm{m}^{2}, \lambda_{e x c}=365 \mathrm{~nm}\right)$ of CLP4 aggregates, film thickness $9 \mathrm{~nm}$, on muscovite mica for increasing substrate temperatures: (a) $300 \mathrm{~K}$, (b) $320 \mathrm{~K}$, (c) $340 \mathrm{~K}$, (d) $360 \mathrm{~K}$, (e) $370 \mathrm{~K}$. 
Again length, width and height depend on the substrate temperature during deposition as found for $\mathrm{p} 6 \mathrm{P}$ and MOP4, too. But the temperature additionally has a pronounced influence on the aggregates' shape. For substrate temperatures close to room temperature the nanostructures consist of straight needles and up to $500 \mathrm{~nm}$ tall aggregates of $\mu \mathrm{m}^{2}$ area (low temperature case). These are usually situated at the end of fibers and form star-shaped entities. The mean height of the needles increases with substrate temperature from $80 \mathrm{~nm}$ to $150 \mathrm{~nm}$, but the number of star-shaped entities decreases with temperature, until from $T_{S} \sim 360 \mathrm{~K}$ on mostly parallely oriented needles with homogeneous heights form (high temperature case). For somewhat higher substrate temperatures $\left(\mathrm{T}_{S}=400\right.$ K) no nanofibers are obsorved at all. Fig 4.7 shows fluorescence microscopy images from five samples grown at different substrate temperatures and Fig 4.8 corresponding atomic force microscopy images.
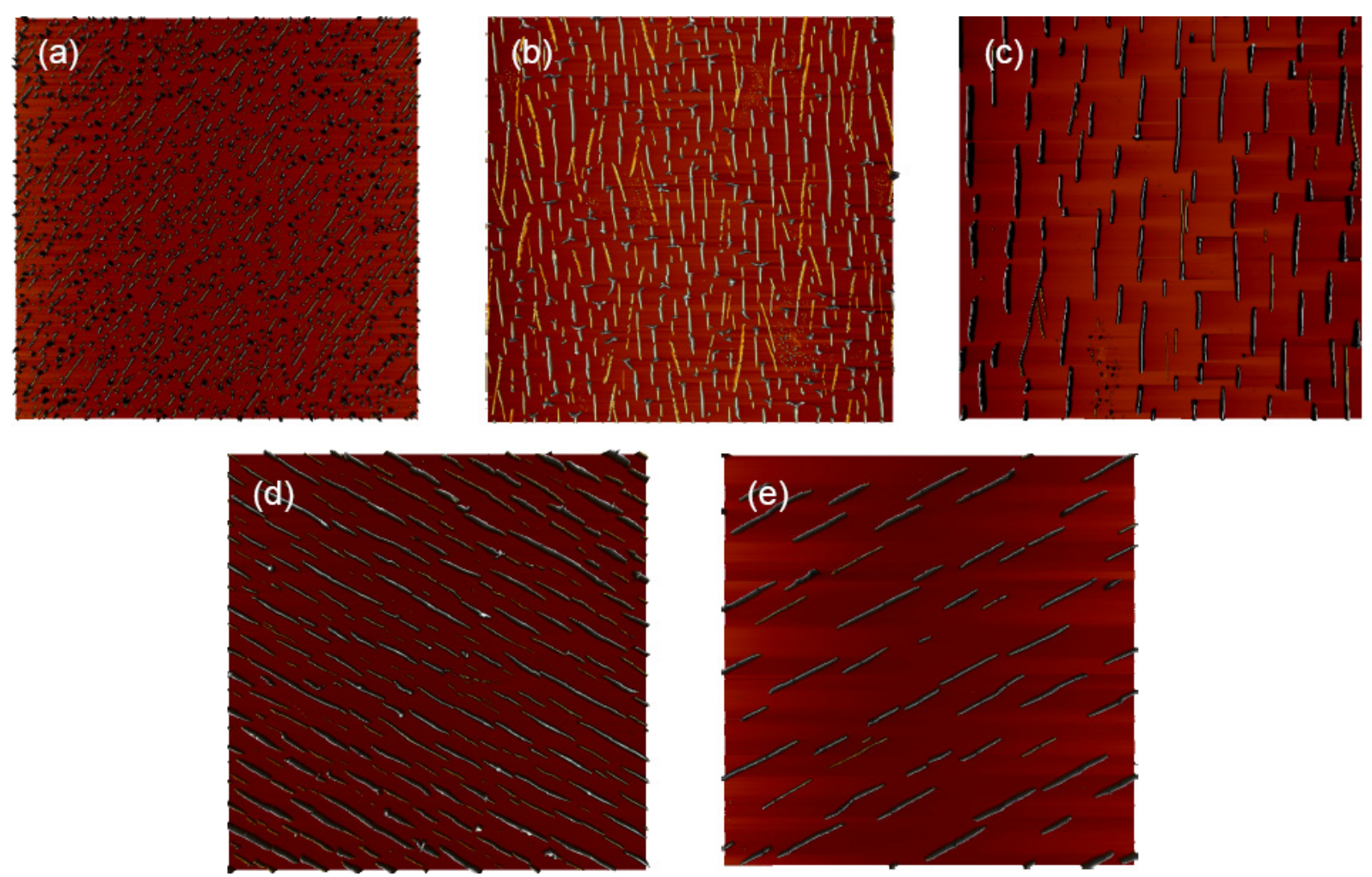

Fig. 4.8: Atomic microscopy images $\left(80 \times 80 \mu \mathrm{m}^{2}\right.$, height scales $\left.150 \mathrm{~nm}\right)$ of CLP4 aggregates on muscovite mica, film thickness $9 \mathrm{~nm}$, for increasing substrate temperatures: (a) $300 \mathrm{~K}$, (b) $320 \mathrm{~K}$, (c) $340 \mathrm{~K}$, (d) $360 \mathrm{~K}$, (e) $370 \mathrm{~K}$.

The number density $N$ of the aggregates decreases with increasing substrate temperatures for constant nominal film thicknesses from $N=3.3 \cdot 10^{7} \mathrm{~cm}^{-2}$ for a substrate temperature close to room temperature down to $N=1.2 \cdot 10^{6} \mathrm{~cm}^{-2}$ for $T_{S}=370 \mathrm{~K}$, whereas the mean length of individual aggregates increases from about $1 \mu \mathrm{m}$ to $10 \mu \mathrm{m}$ with single fibers up to $30 \mu \mathrm{m}$, Fig 4.9 . 

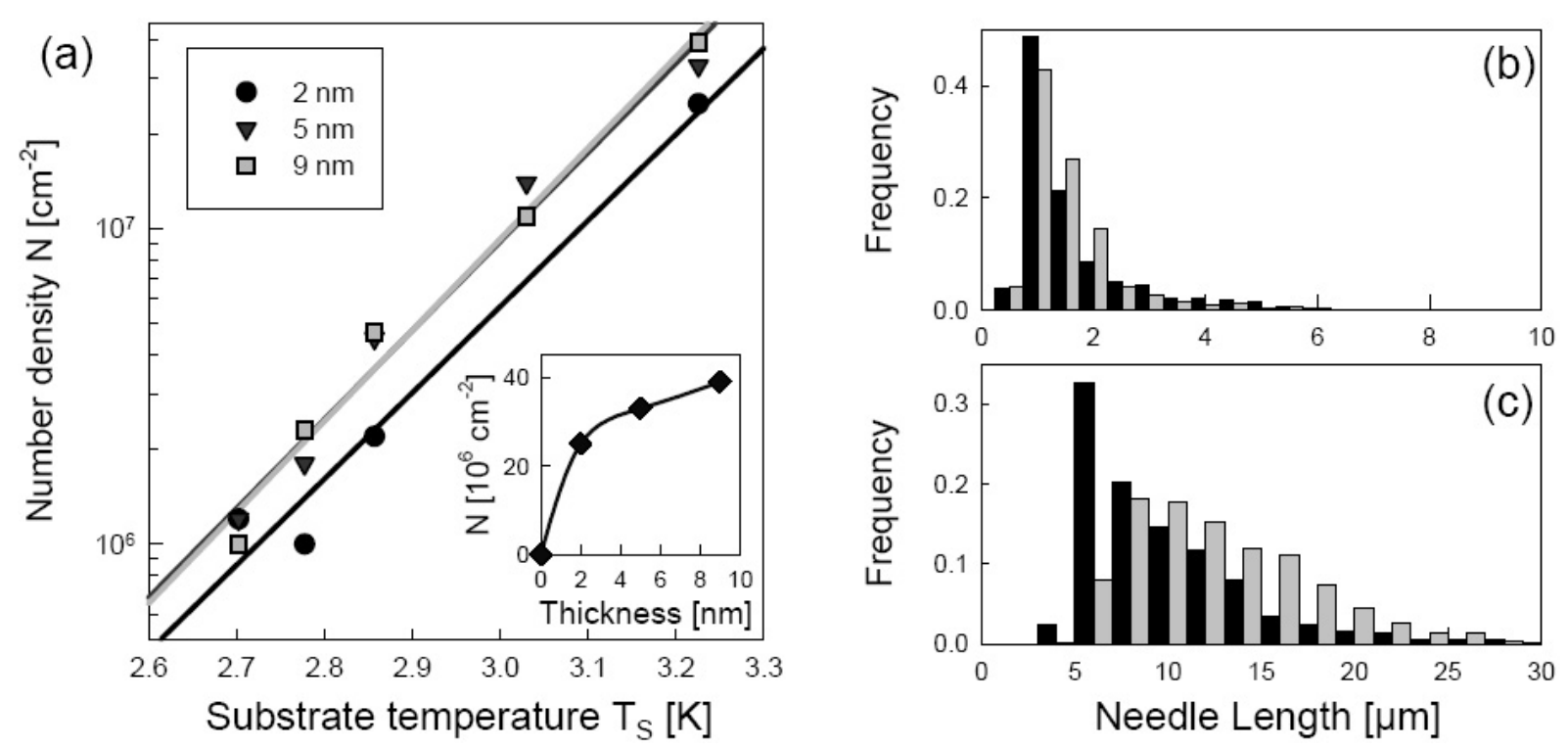

Fig. 4.9: (a) Number density $N$ of CLP4 aggregates on mica as a function of the reciprocal substrate temperature $1 / \mathrm{T}_{S}$ for samples with film thicknesses of $2 \mathrm{~nm}$ (circles), 5 $\mathrm{nm}$ (triangles), and $9 \mathrm{~nm}$ (squares). Straight lines depict linear regression for the different sample thicknessess. The inset shows the thickness dependence of $N$ for $\mathrm{T}_{S}=310 \mathrm{~K}$. The length distribution of needles for deposition (b) at room temperature, and (c) at $\mathrm{T}_{S}=370$ $\mathrm{K}$ demonstrates a strong increase of needle length with $\mathrm{T}_{S}$. The black bars correspond to a nominal film thickness of $2 \mathrm{~nm}$, the grey bars to $9 \mathrm{~nm}$ of deposited CLP4. From [5].

As a function of substrate temperature $T_{S}$ during deposition an Arrhenius type behaviour for the aggregate number density $N$ has been observed, $N \propto \exp \left(E_{N} / k T_{S}\right)$ with an activation energy $E_{N} \approx 0.56 \pm 0.1 \mathrm{eV}$, cf. the linear regression in Fig.4.9(a). A similar value being in order of few hundred $\mathrm{meV}$ has been found e.g. for sexithiophene islands on mica [164]. For homogeneous nucleation such a behavior has been predicted in the literature, with the activation energy $E_{N}$ being a function of characteristic energies like the energies for adsorption and diffusion and of the critical nucleus size of the system [165]. Similar to the case of $\mathrm{p} 6 \mathrm{P}$ the needles are presumably not the initial aggregates formed. Three dimensional clusters in between the needles have been observed in AFM images for the CLP4 samples grown at elevated substrate temperatures. Needles probably grow by agglomeration of such clusters [166]. However, for a reasonably homogeneous size distribution of the needles the needle density should still somewhat reflect the distribution of the primary islands.

It can clearly be distinguished between a low temperature and a high temperature case. For both cases the number density of aggregates increases with increasing film thicknesses up to a nominal thickness of $5 \mathrm{~nm}$, beginning to saturate afterwards. For temperatures close to room temperature, the low temperature case, the dominating effect is a strong 

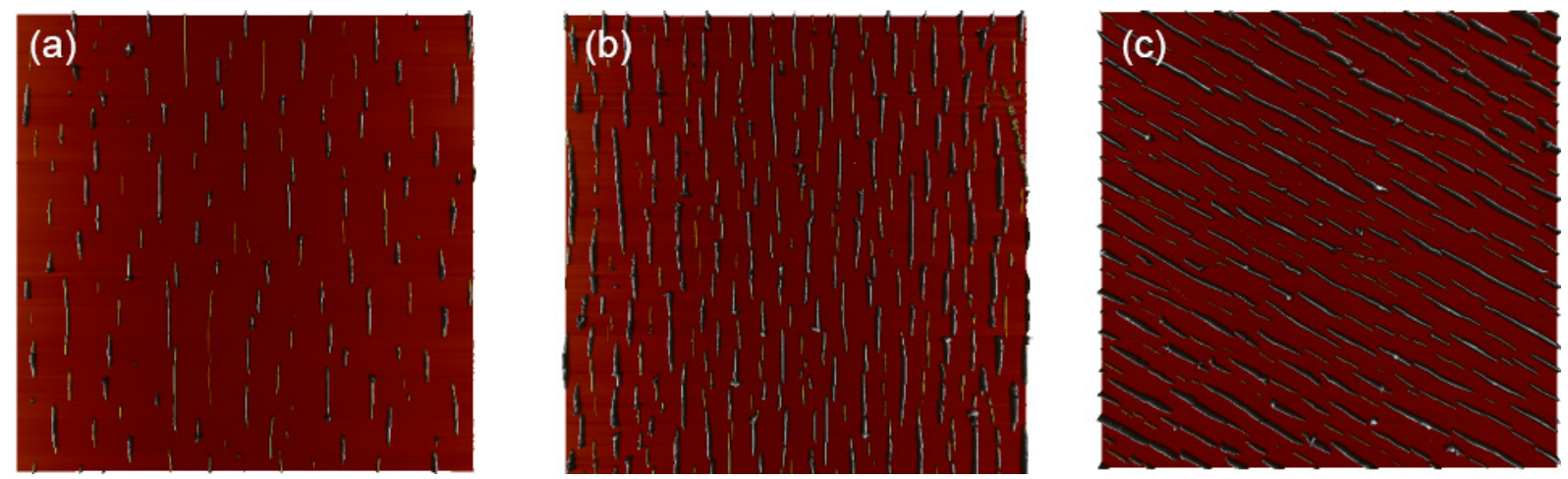

Fig. 4.10: Atomic microscopy images $\left(80 \times 80 \mu \mathrm{m}^{2}\right.$, height scales $\left.120 \mathrm{~nm}\right)$ of CLP4 aggregates on muscovite mica for increasing nominal film thicknesses: (a) $2 \mathrm{~nm}$, (b) $5 \mathrm{~nm}$, (c) $9 \mathrm{~nm}$. Substrate temperature during deposition was 340 K. From [5].
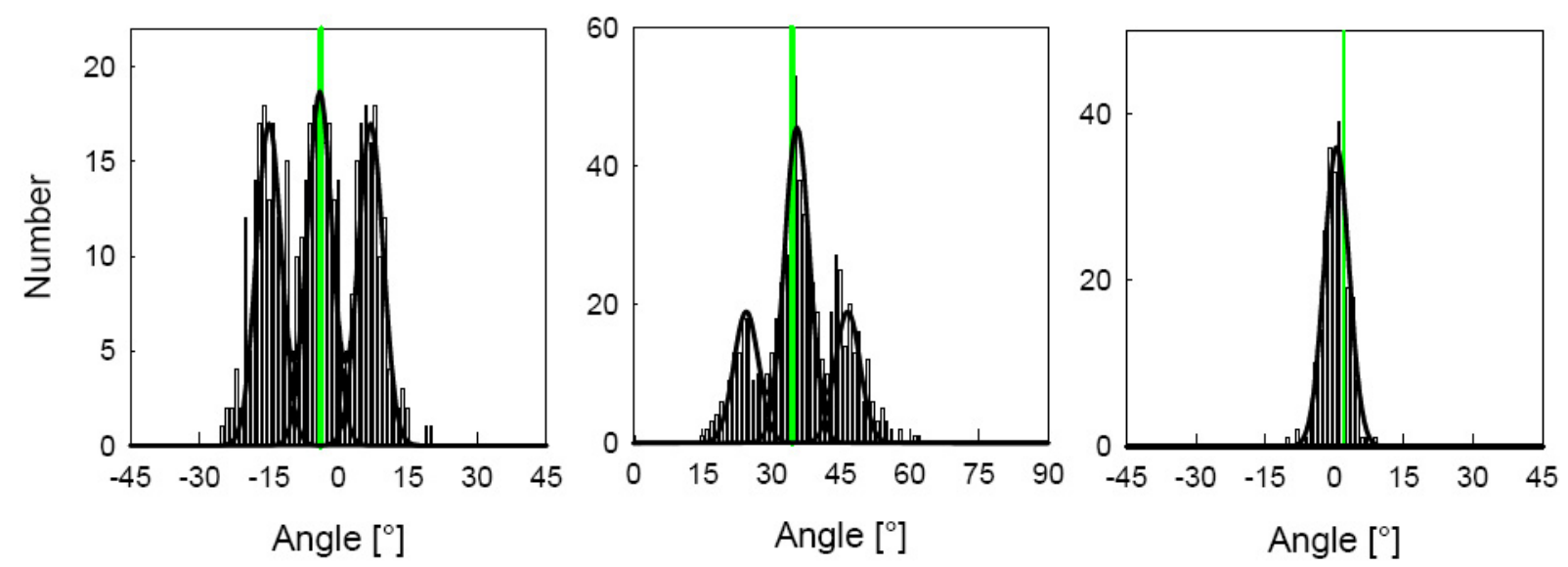

Fig. 4.11: For three different substrate temperatures, left $T_{S}=300 \mathrm{~K}$, middle $340 \mathrm{~K}$ and right $360 \mathrm{~K}$, distributions of needle orientations obtained from AFM and fluorescence microscope images are shown. The vertical lines at $\Delta \phi=0^{\circ}$ indicate the $\langle 110\rangle$ mica orientation. Within the experimental error of $\pm 3^{\circ}$ the main needle direction is along muscovite $\langle 110\rangle$, with two additional directions at $\Delta \phi= \pm 11^{\circ}$ at low deposition temperatures. The solid lines are Gaussian fits to the experimental distributions $\left(\mathrm{FWHM}=6^{\circ}\right)$. From [5].

increase in height of the star-shaped entities reaching heights up to $500 \mu \mathrm{m}$ for $9 \mathrm{~nm}$ nominal film thickness. Fibers increase only slightly in lengtg with a mean length below $2 \mu \mathrm{m}$. Also for higher temperatures the increase in length with increasing nominal film thicknesses is marginal. Newly deposited organic material mainly augments the number density and the height of the needles, Fig 4.10 and inset Fig 4.9(a). Obviously an appropiate substrate temperature is more efficient in producing long fibers than the overall film thickness. The high temperature case is favourable if one aims at straight, parallelly aligned nanoneddles. 
Not only the shape, but also the mutual alignment of the CLP4 fibers strongly depends on the growth temperature. For the low surface temperature case the needles exhibit three orientations within one domain (Fig.4.7(a),(b) and Fig.4.8(a),(b)). Needles grow along $\langle 110\rangle$ and along two directions about $\Delta \phi= \pm 11^{\circ}$ off $\langle 110\rangle$, the off-needles. For increased substrate temperature during deposition the number of off-needles decreases (Fig 4.7(c) and Fig $4.8(\mathrm{c})$ ), until almost all needles form along $\langle 110\rangle$ at $T_{S} \geq 350 \mathrm{~K}$ (Fig.4.7(d),(e) and Fig. $4.8(\mathrm{~d}),(\mathrm{e}))$.
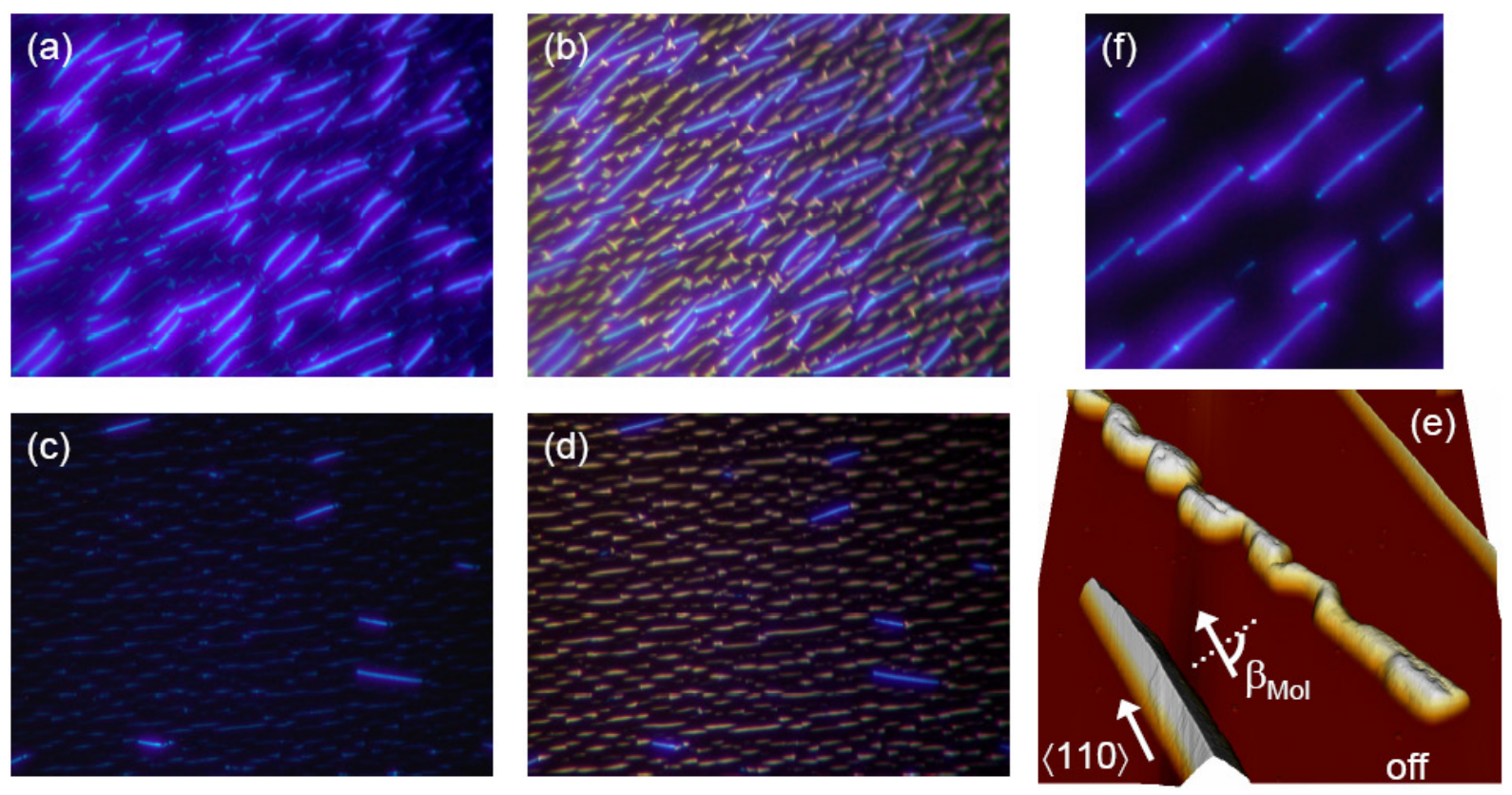

Fig. 4.12: CLP4 on muscovite mica: substrate temperature during deposition $T_{S}=320$ $\mathrm{K}$, image sizes $113 \times 85 \mu^{2}$ (a) fluorescence microscopy image and (b) combination of darkfield and fluorescence microscopy image; substrate temperature during deposition $T_{S}$ $=340 \mathrm{~K}$, image sizes $160 \times 120 \mu \mathrm{m}^{2}$ (c) fluorescence microscopy image and (d) combination of darkfield and fluorescence microscopy image. The AFM image (e) $\left(5 \times 5 \mu \mathrm{m}^{2}\right.$, height scale $100 \mathrm{~nm} ; T_{S}=340 \mathrm{~K}$ ) shows the detailed morphology of the brighter fluorescent off-needles and the $\langle 110\rangle$-needles. The angle $\beta_{\text {Mol }}$ is the relative angle between plane of polarisation and needle direction (see text for details). The fluorescence microscopy image (f) $\left(55 \times 55 \mu^{2}\right)$ shows CLP4-fibers from the high surface temperature case, $T_{S}$ $=370 \mathrm{~K}$.

The overall fluorescence intensity for normal incidence observation is different for the $\langle 110\rangle$-needles and for the off-needles. Fibers exactly along $\langle 110\rangle$ emit three to four times less fluorescence per unit length than the off-direction fibers. Combining fluorescence and darkfield imaging visualises clearly both needle types. For a substrate temperature during deposition of $320 \mathrm{~K}$, the low surface temperature case, the off-fibers dominate the 
fluorescence microscopy image (Fig 4.12(a)). The combined fluorescence and darkfield image (b) shows, that the less fluorescent aggregates consist of comparatively short $\langle 110\rangle$ fibers ending partially in star-shaped entities. For a slightly higher substrate temperature, namely $T_{S}=340 \mathrm{~K}$, the number of off-fibers decrease. The $\langle 110\rangle$-fibers dominate the sample with increased length compared to the ones for the low temperature case. Hardly any star-shaped entities are visible (Fig.4.12(c),(d)).

However, the polarisation dependence of the emitted light after excitation under normal incidence and under normal observation shows no difference for the two needle types. Both for the $\langle 110\rangle$-needles as well as for the off-needles the angular distribution of fluorescence can be fitted by Malus law (see Chapter Experimental) and shows a maximum in intensity for light polarised perpendicular to $\langle 110\rangle \pm 3^{\circ}$, i.e. perpendicular to the needle direction for the $\langle 110\rangle$-needles $\left(\beta_{\mathrm{Mol}}=90^{\circ}\right)$ and at $\beta_{\mathrm{Mol}}=77^{\circ} \pm 5^{\circ}$ for the off-needles (Fig 4.12 . Assuming a herringbone packing of the molecules, different angles $\beta_{\text {Mol }}$ can easily be explained by different contact faces of the bulk like needles with the substrate. For lying molecules with the $\left(\begin{array}{lll}0 & 0\end{array}\right)$ contact plane (or similar planes with just different tilt angles of the molecules with respect to the substrate) a value of $\beta_{\mathrm{Mol}}=90^{\circ}$ would result, for the (110)-type of planes $\beta_{\mathrm{Mol}}=76^{\circ}$, i.e. the observed values. For p6P these two needle types have indeed been observed [38, 58].

An AFM image of an off-needle and of two $\langle 110\rangle$-needles (Fig $4.12(\mathrm{e})$ ), discloses that the two fiber types have quite different morphologies. The $\langle 110\rangle$-needles are straight and show clear facets, whereas the off-needles have a rugged morphology. Heights and widths, however, are similar to the straight fibers. The reason for the higher luminescence efficiency therefore is not due to more material within the needle, but might result from a different crystal structure with the same lateral orientation of the molecules with respect to the substrate. This would, e.g., imply that the molecules are more parallel to the substrate for the rugged than for the straight fibers.

Another possible explanation are different waveguiding and light scattering properties for the two needle-types. The $\langle 110\rangle$-fibers from the high surface temperature case exhibit brightly fluorescent spots within the fibers and at the endings (Fig $4.12(f))$. The bright spots within a fiber originate from breaks, which can be clearly seen by atomic force microscopy. In case of $\mathrm{p} 6 \mathrm{P}$ it has been demonstrated, that the fibers act as waveguides [46 48] and the light is scattered at breaks within the fiber. According to this the $\langle 110\rangle$ needles from the CLP4 are believed to show pronounced waveguiding phenomena. The generated fluorescence light upon UV-excitation can be kept inside the well shaped fiber and waveguided to breaks and endings before it is scattered. Therefore only spots appear very bright whereas the main body of the fibers seem to be less fluorescent. The off-needles may not function as waveguides due to inhomogenity of the fiber's shape. Generated fluorescence light can be scattered at every unevenness, light is not kept within the fiber. So rugged off-needles exhibit a homogeneously fluorescence intensity along the whole fiber. 


\subsubsection{Di-Cyano- $p$-Quaterphenylene (CNP4)}

Deposition of di-cyano functionalised p-quaterphenylene CNP4 onto a freshly cleaved muscovite mica substrate leads to formation of diverse structures within a single domain. Beside mutually aligned fiber-like structures aggregates with increased heights are visible with a shape that reminds of swallow wings. According to the here shown images for a substrate temperature $T_{S}=300 \mathrm{~K}$ and film thickness $8 \mathrm{~nm}$, the fibers have a mean length of about $8 \mu \mathrm{m}$ as well as a typical width of several $100 \mathrm{~nm}$, and height up to 100 nm. A single swallow wing exhibits a mean length of $4 \mu \mathrm{m}$ and a width in the same dimension as the fiber-like structures but the height is up to $200 \mathrm{~nm}$, i.e. about twice as high compared to the fibers. The fibers grow along the $\langle 110\rangle$ muscovite direction whereas the swallow wings grow along $\langle 110\rangle$ (but rotated by $120^{\circ}$ with respect to the fibers) as well as along $\left[\begin{array}{lll}1 & 0 & 0\end{array}\right]$. CNP4 is the only one among the functionalised $p$-quaterphenylenes tested so far, which shows not only growth along both muscovite $\langle 110\rangle$ directions within a single domain but also along muscovite $\left[\begin{array}{lll}1 & 0 & 0\end{array}\right]$.
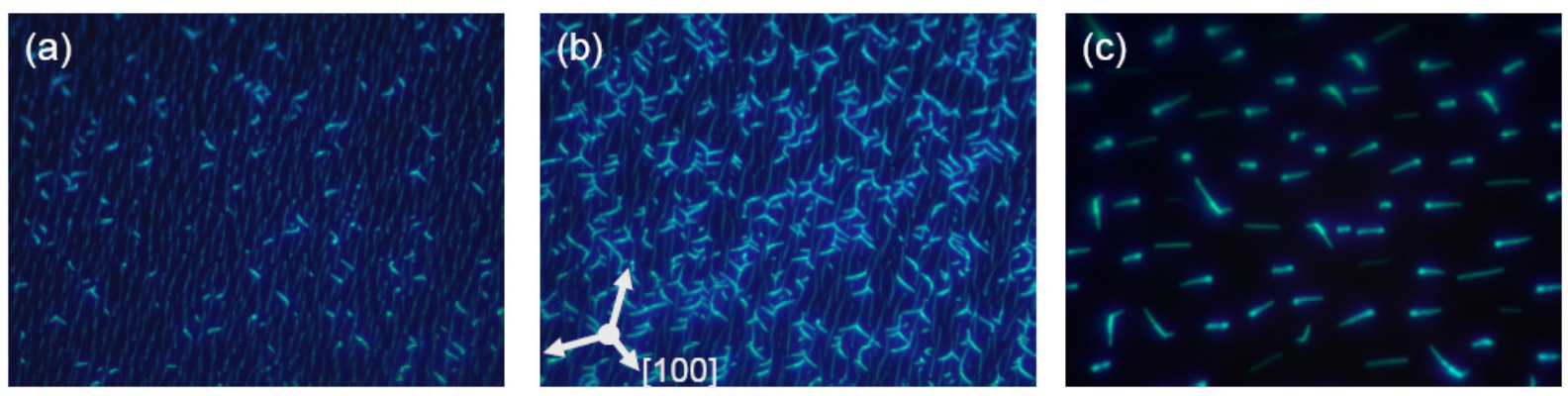

Fig. 4.13: Fluorescence microscopy images $\left(\lambda_{(e x c)}=365 \mathrm{~nm}\right)$ of CNP4 on muscovite mica for different nominal film thicknesses and substrate temperatures: (a) $4 \mathrm{~nm}$, (b) 8 $\mathrm{nm}$, both $T_{S}=300 \mathrm{~K}, 113 \times 85 \mu \mathrm{m}^{2}$, and (c) $T_{S}=360 \mathrm{~K}, 56 \times 42 \mu \mathrm{m}^{2}, 8 \mathrm{~nm}$.

Both nanofiber types emit polarised blue light in the fluorescence microscope with the polarisation vector pointing in the same direction, i.e. almost perpendicular to the long axes of the $\langle 110\rangle$ fibers. Since the molecular orientation determines the polarisation properties the oligomers are orientated in the same direction for the fibers and the swallow wings. That way the different aggregates reflect different crystallographic properties, either varying crystal structures or varying crystal faces being parallel to the substrate. However, the fluorescence light is always polarised almost perpendicular to the grooved muscovite $\langle 110\rangle$, which means that the oligomers orientate with their long molecular axes along the surface electric fields. Two different domains are visible on a single sample, mutually rotated by $120^{\circ}$.

The shape of the nanostructures is not strongly dependent on the growth temperature, i.e. over the whole tested substrate temperature range fibers and swallow wings are visible. First for lower coverages the fibers along grooved muscovite $\langle 110\rangle$ form, only 

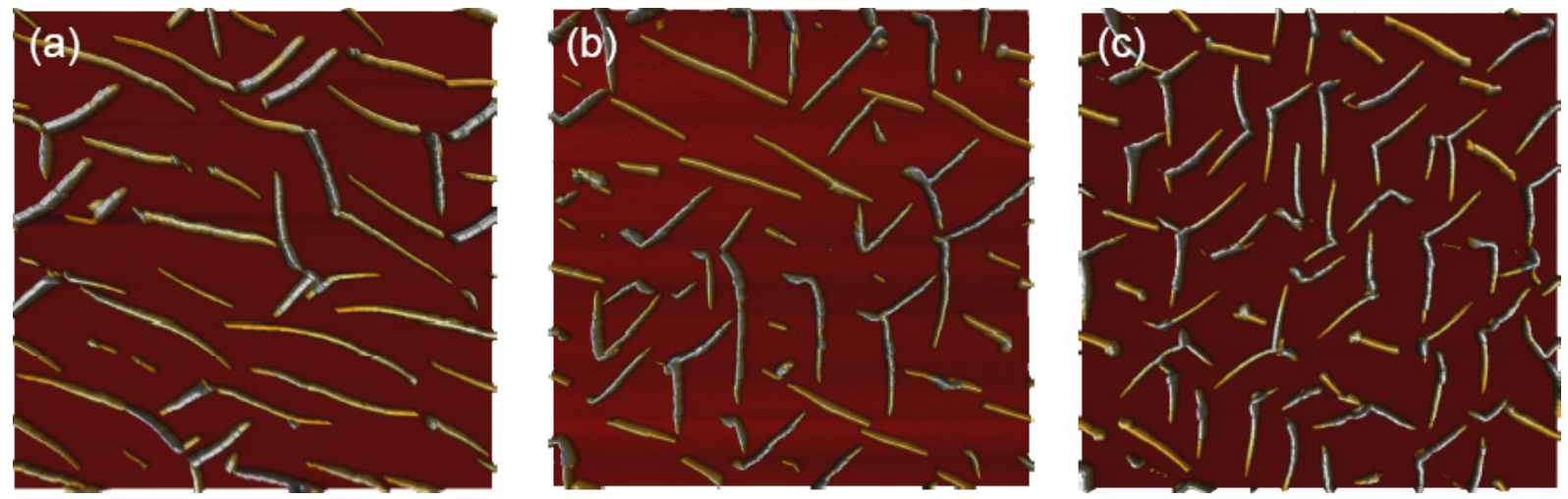

Fig. 4.14: Atomic force microscopy images of CNP4 on muscovite mica for different substrate temperatures: (a) $T_{S}=300 \mathrm{~K}$, (b) $T_{S}=310 \mathrm{~K}, 5 \mathrm{~nm}$, and (c) $T_{S}=320 \mathrm{~K}, 8$ $\mathrm{nm}$ film thickness; all $20 \times 20 \mu \mathrm{m}^{2}$, height scales $160 \mathrm{~nm}$.

a few swallow wings are visible. For increasing film thicknessess the number of swallow wings increases (Fig 4.13). The swallow wings show brighter fluorescence due to more organic material since they are significantly higher than the fibers.

For the same nominal film thickness but higher substrate temperature the nanostructure of both types are significantly shorter. The decrease in length is more pronounced for the $\langle 110\rangle$ fibers. According to only the AFM images Fig 4.14 the mean length decreases from about $8 \mu \mathrm{m}\left(T_{S}=300 \mathrm{~K}\right)$, over about $5 \mu \mathrm{m}\left(T_{S}=310 \mathrm{~K}\right)$, to about $3 \mu \mathrm{m}\left(T_{S}=320 \mathrm{~K}\right)$. Interestingly in cases of p6P, MOP4 and CLP4 an increase of substrate temperature (up to a certain maximun) leads to longer nanofibers!

Due to the reduced mean length the same number of nanoaggragates (fibers and swallow wings) is on the same area, i.e. the aggegate number density $N$ is the same for Fig. 4.14(a), $T_{S}=300 \mathrm{~K}$ and Fig $4.14(\mathrm{~b}), T_{S}=310 \mathrm{~K}$, namely $1.1 \times 10^{7} \mathrm{~cm}^{-2}$ (both $5 \mathrm{~nm}$ film thickness). $N$ increases slightly for Fig 4.14 (c), $T_{S}=320 \mathrm{~K}$ to $1.3 \times 10^{7} \mathrm{~cm}^{-2}$ because of more deposited organic material ( $8 \mathrm{~nm}$ film thickness). Experimental error is $\pm 0.2 \times 10^{7}$ $\mathrm{cm}^{-2}$.

\subsubsection{Di-N,N-Dimethyl-Amino- $p$-Quaterphenylene (NMeP4)}

Vapour deposition of NMeP4 on muscovite mica at elevated substrate temperatures results in the formation of well shaped nanoaggregates. Again their shape is strongly dependent on the subtrate temperature during deposition. In this case, however, the low temperature case, $T_{S}=340 \mathrm{~K}$, leads to the needle-like aggregates in opposition to CLP4, which needs the high substrate temperature case for generating parallely orientated nanofibers.

The low temperature nanofibers from NMeP4 look slightly bent like worms, Fig.4.15(a). They posses a mean length of about $10 \mu \mathrm{m}$ as well as a typical width of a few $100 \mathrm{~nm}$ and height of several $10 \mathrm{~nm}$, respectively. The growth direction is along muscovite $\langle 110\rangle$ as 

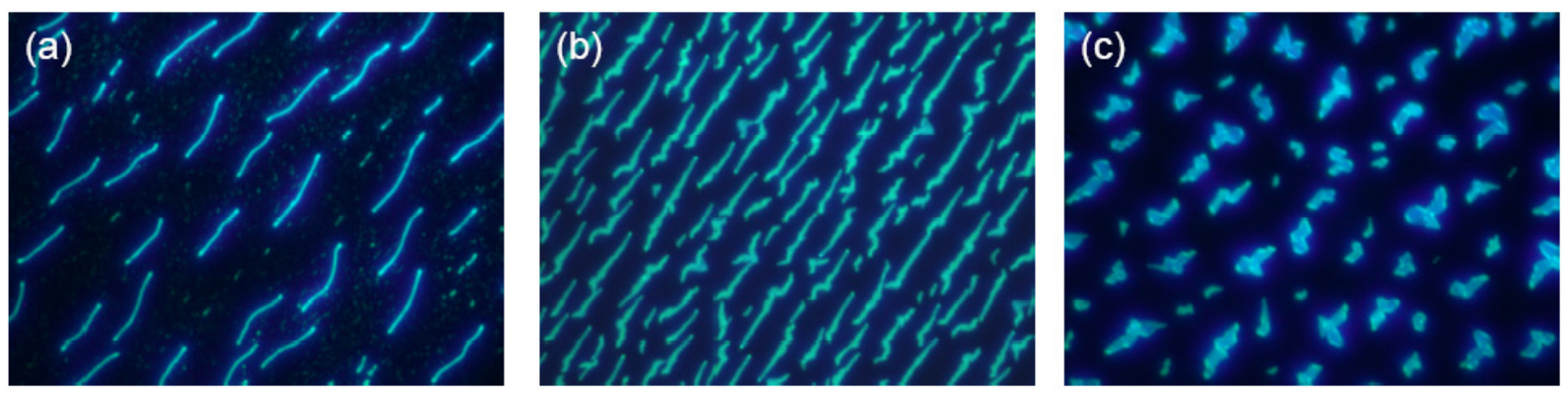

Fig. 4.15: Fluorescence microscopy images of NMeP4 on muscovite mica for different substrate temperatures: (a) $T_{S}=340 \mathrm{~K}, 7 \mathrm{~nm}$ (b) $T_{S}=380 \mathrm{~K}, 10 \mathrm{~nm}$, and (c) $T_{S}=400$ $\mathrm{K}, 10 \mathrm{~nm}$; all $56 \times 42 \mu \mathrm{m}^{2}$.

usual. In between the nanoworms small poorly fluorescent aggregates are visible. Atomic force microscopy images reveal (Fig 4.16(a)) that the space between the nanoworms is densely filled with small, elongated clusters. These hardly fluoresce due to their littleness compared to the nanoworms.
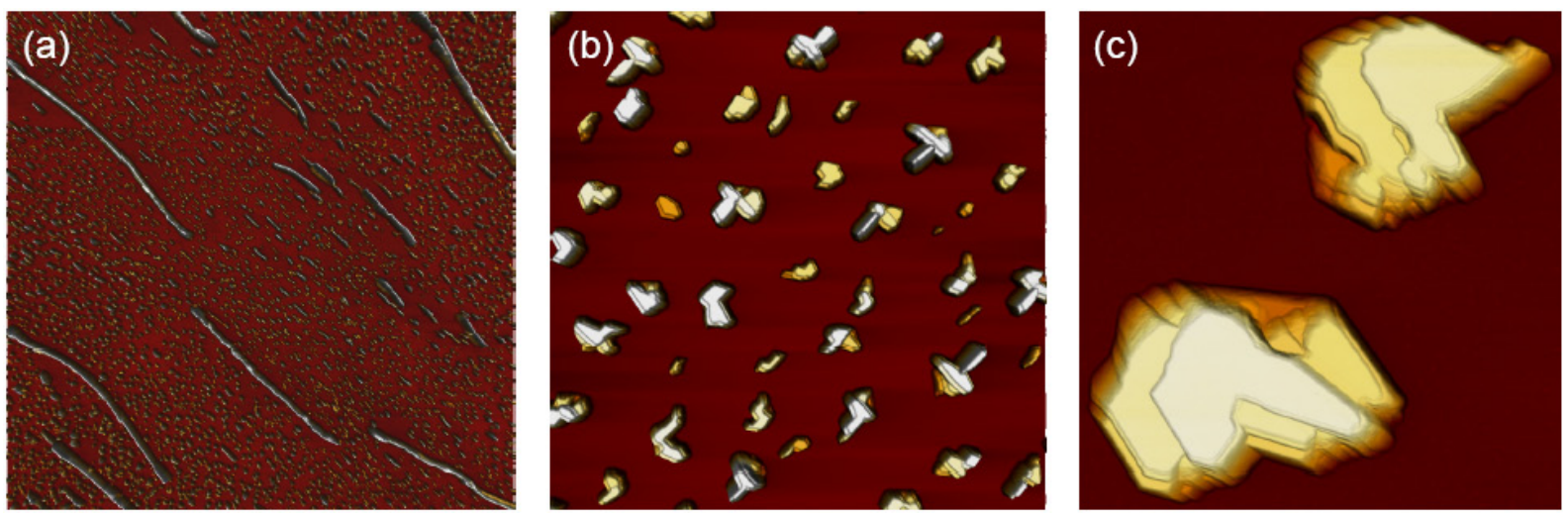

Fig. 4.16: Atomic force microscopy images of $\mathrm{NMeP} 4$ on muscovite mica for different substrate temperatures: (a) $T_{S}=340 \mathrm{~K}, 7 \mathrm{~nm}, 15 \times 15 \mu \mathrm{m}^{2}$, height scale $120 \mathrm{~nm}$ (b) 35 $\times 35 \mu \mathrm{m}^{2}$, and (c) $5 \times 5 \mu \mathrm{m}^{2}$, both $T_{S}=400 \mathrm{~K}, 10 \mathrm{~nm}$, height scale $220 \mathrm{~nm}$.

For higher substrate temperatures, $T_{S}=380 \mathrm{~K}$, the medium temperature case, the nanoaggregates look similar to pollywogs, Fig 4.15(b), oriented along muscovite $\langle 110\rangle$. For increasing nominal film thicknesses the pollywogs gain clearly in length, height and width according to Fig.4.17. The mean length starting from about $2 \mu \mathrm{m}$ (film thickness $2 \mathrm{~nm}$ ) increases over $4 \mu \mathrm{m}$ (film thickness $4 \mathrm{~nm}$ ) to about $8 \mu \mathrm{m}$ (film thickness $10 \mathrm{~nm}$ ), Fig 4.18(a). The nanostructures possess heights of about $100 \mathrm{~nm}$ (film thickness $2 \mathrm{~nm}$ ), $120 \mathrm{~nm}$ (film thickness $4 \mathrm{~nm}$ ) and $140 \mathrm{~nm}$ (film thickness $10 \mathrm{~nm}$ ) respectively, Fig 4.18(b). The width can be more than $1.5 \mu \mathrm{m}$. For nanostructures wider than about $800 \mathrm{~nm}$, the top surface is flat and even.

For substrate temperatures around $400 \mathrm{~K}$, the high temperature case, the nanoaggre- 

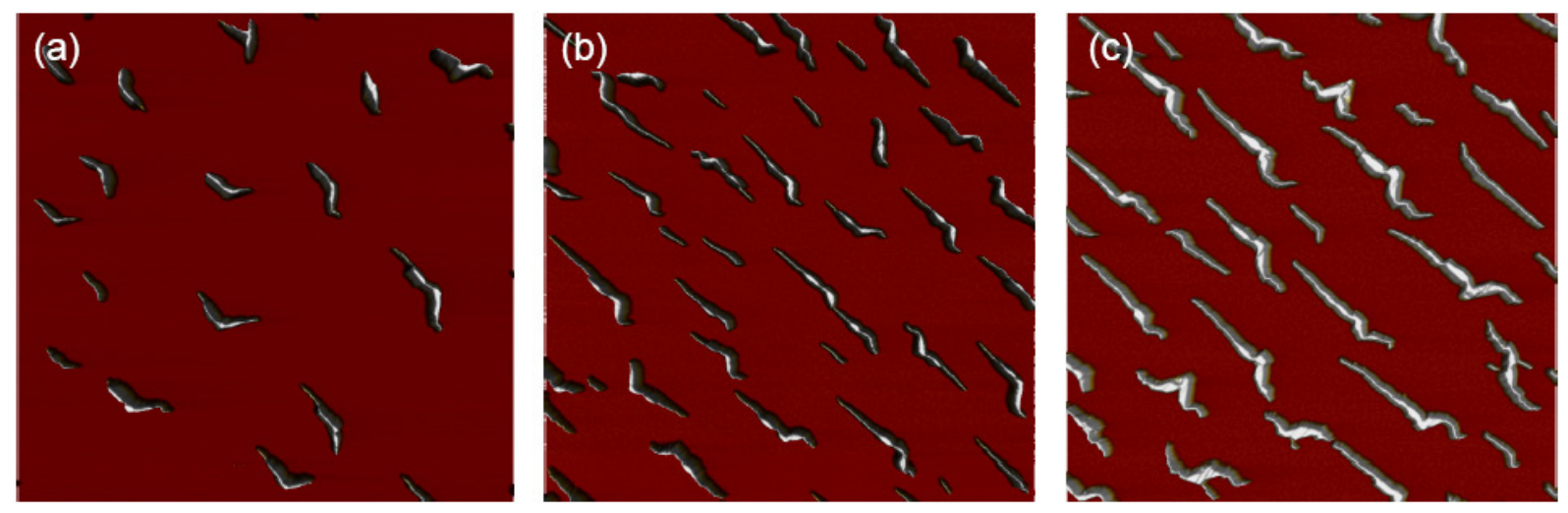

Fig. 4.17: Atomic force microscopy images of NMeP4 on muscovite mica $\left(T_{S}=380 \mathrm{~K}\right)$ for different nominal film thicknesses: (a) $2 \mathrm{~nm}, 20 \times 20 \mu \mathrm{m}^{2}$ (b) $4 \mathrm{~nm}, 25 \times 25 \mu \mathrm{m}^{2}$ and (c) $10 \mathrm{~nm}, 25 \times 25 \mu \mathrm{m}^{2}$; height scales $100 \mathrm{~nm}$.
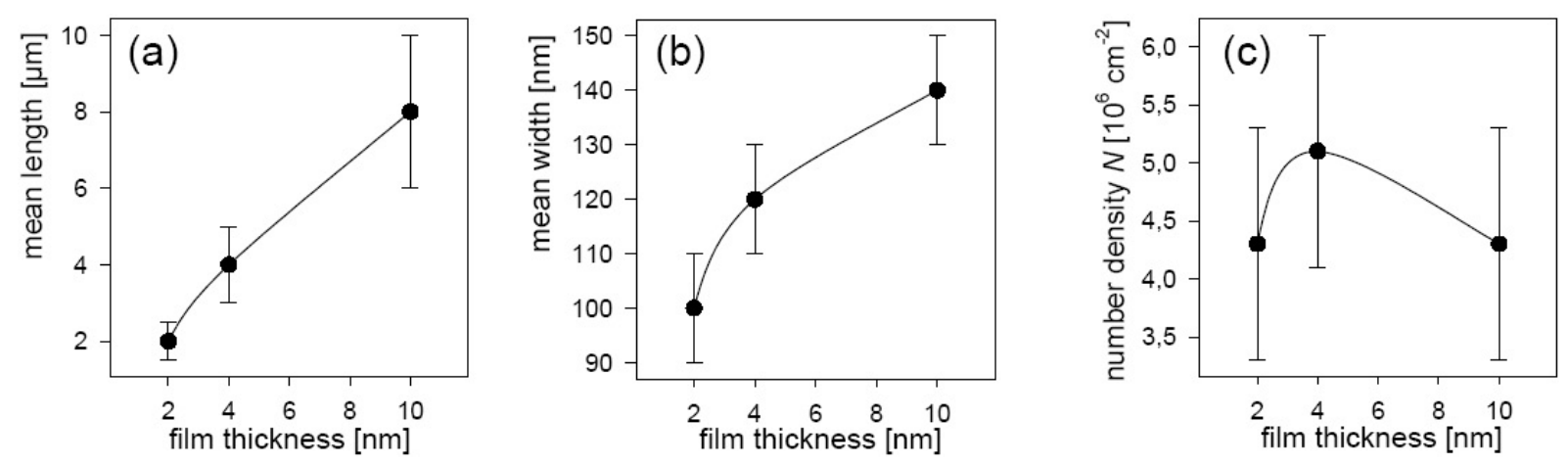

Fig. 4.18: Statistics on the medium temperature case, $T_{S}=380 \mathrm{~K}$, of NMeP4's nanoaggregates: (a) mean length, (b) mean width, and (c) aggregate number density $N$ according to Fig. 4.17 .

gates look like flakes. They are well separeted and hardly prefer any growth direction. But the flakes show the same polarisation properties as the fiberlike nanostructures indicating a highly crystalline structure. The flakes reach size of about $3 \times 3 \mu \mathrm{m}^{2}$ and about $200 \mathrm{~nm}$ in height. The top-surface is exceedingly flat and even exhibiting few well shaped steps with a height of a few ten nanometer.

Only for the low temperature case, $T_{S}=340 \mathrm{~K}$, elongated clusters are visible between the nanoworms. For higher substrate temperatures these clusters seem to be eaten up by the nanoaggregates due to increased surface mobility at elevated temperatures. The high temperature case shows regular patterns of flake-like islands with a diameter of several ten nanometer (not visible in the AFM-images shown here). A step-height of $2.5 \mathrm{~nm}$ indicates upright standing molecules on the surface. In contrast the elongated clusters are built up of lying molecules because they show fluorescence.

However, the generation of well defined nanostructures from NMeP4 demonstrates, that even the comparatively bulky and highly polar $N, N$-dimethyl amino group is accepted 
within the growth process. A strong dependence of the nanostructures ${ }^{6}$ morphology on the substrate temperature during deposition is observed similar to CLP4. Interestingly the low temperature case leads to formation of elongated nanofibers (i.e. nanoworms) in case of NMeP4 opposite to CLP4 with the high temperature case being favourable for generating parallely aligned nanofibers. Since also p6P and MOP4 form longer nanofibers for increasing substrate temperatures (up to a certain limit), the behaviour of NMeP4 can be described as inverted temperature dependence. Interestingly this has also been observed for CNP4's nanostructures. Such behaviour is contrary to basic nucleation theory [167], which predicts larger and more separated crystallites for higher substrate temperatures, as long as no degradations of the entire aggregates takes place, as described in detail for p6P on muscovite mica [63, 69].

\subsubsection{Di-Amino- $p$-Quaterphenylene (NHP4)}

The di-amino functionalised $p$-quaterphenylene NHP4 self-assembles into mutually aligned fiberlike nanostructures.
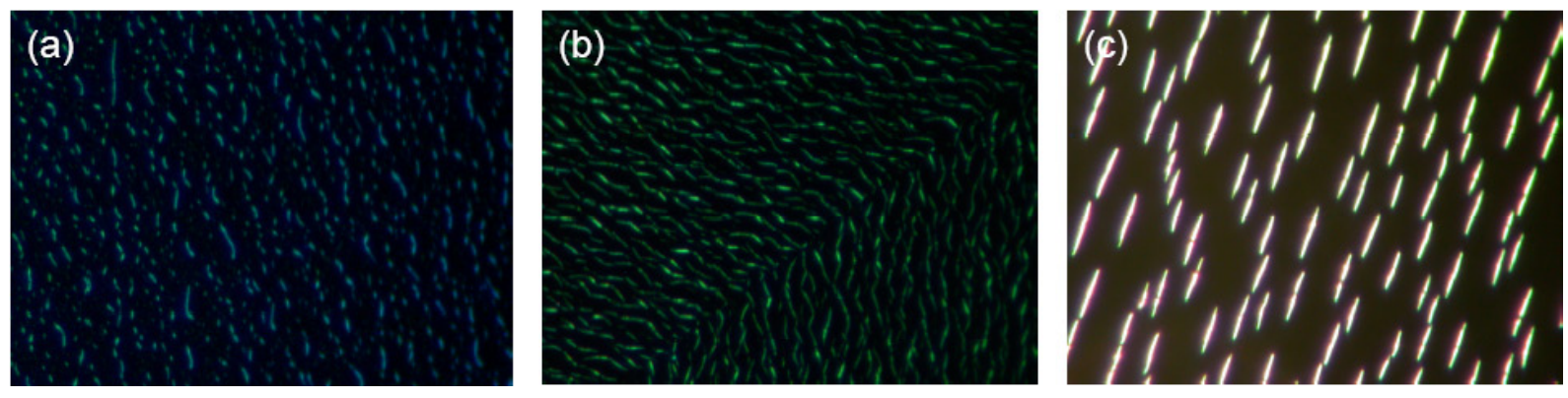

Fig. 4.19: Fluorescence microscopy images of NHP4 on muscovite mica for different substrate temperatures and film thicknesses: (a) $T_{S}=380 \mathrm{~K}, 4 \mathrm{~nm}$, (b) $T_{S}=400 \mathrm{~K}, 10$ $\mathrm{nm}$, and a darkfield microscopy image (c) $T_{S}=430 \mathrm{~K}, 6 \mathrm{~nm}$; all images $113 \times 85 \mu \mathrm{m}^{2}$.

For relativly low substrate temperatures, $\mathrm{T}_{S}=380 \mathrm{~K}$ short, wormlike aggregates with a length of only a few micrometer form with clusters in between (Fig.4.19(a)). These NHP4 nanoworms look very similar to the low temperature NMeP4 nanoworms (see above). The clusters in between vanish at elevated substrate temperatures, $\mathrm{T}_{S}=400 \mathrm{~K}$ in favour of the wormed, fiberlike structures (Fig.4.19(b)). Further increase in substrate temperature leads to well separated parallely aligned nanofibers with a length up to $10 \mu \mathrm{m}$ (Fig.4.19(c)).

Compared to NMeP4, which possesses an amino group with the hydrogen substituted by methyl groups, the temperature dependence of the growth of NHP4 (unsubstituted amino group) is completely different: The shape of the nanostructures of NHP4 is not strongly depending on the growth temperature, i.e. no different temperature cases are visible. Only the normal temperature dependence is existent, i.e. the nanostructures show better mutual alignment and enhanced fiber growth for increasing substrate temparatures up to 
a certain limit. The fiberlike nanostructures from both differently amino functionalised oligomers look rather similar for relatively low substrate temperatures: nanoworms are formed with clusters in between.

Another pronounced discrepancy for the two differently amino functionalised oligomers is the fluorescence behaviour. The nanostructures from NHP4 show hardly fluorescence in contrast to all previously discussed nanoaggregates. The fluorescence after excitation with UV light from a high pressure mercury lamp $\left(\lambda_{\text {exc }}=365 \mathrm{~nm}\right)$ vanishes within seconds and in doing so the fluorescence colour changes from blue to green (cf. Fig. 4.19). This phenomenon of fluorescence quenching could be due to intramolecular interactions between the hydrogen of the amino group and the aromatic system of the phenyl rings, or due to intermolecular hydrogen bonding of adjacent amino groups. This has to be clarified in the future. Quenching might not be the proper description, because this quick bleaching of the fluorescence is not reversible. Therefore structure changes on the molecular level are supposed to happen. However, this problem of fluorescence quenching is well known for naphthalene bisimide dyes core-substituted with arylamines: Formation of intramolecular hydrogen bonding to a neighbouring carbonyl group is considered to be the pathway for radiationless deactivation of the excited state. To avoid this problem alkyl amines [168] and amino-linked benzyl groups [169], respectively, have been attached sucessfully to the naphthalene core leading to a strong, tunable fluorescence.
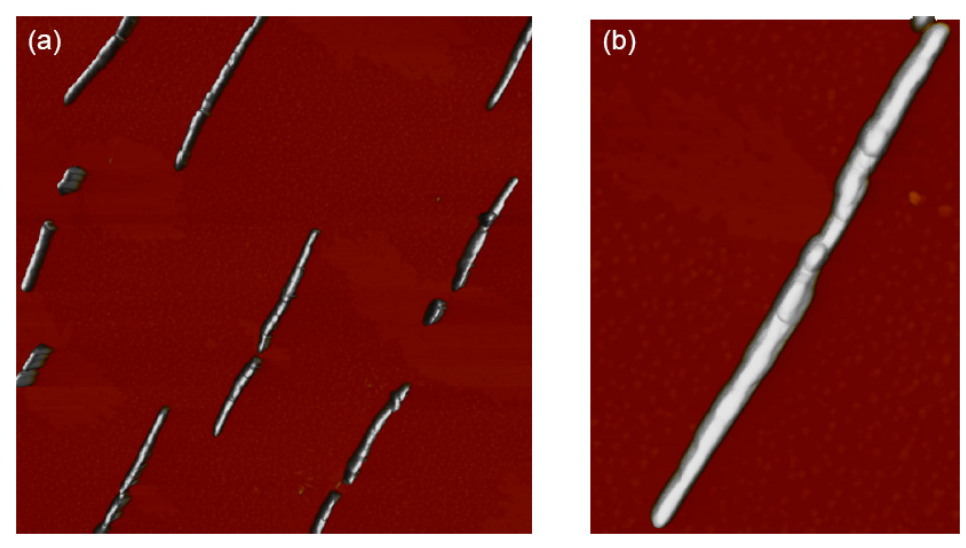

Fig. 4.20: Atomic force microscopy images of NHP4 on muscovite mica $\left(T_{S}=430 \mathrm{~K}, 6\right.$ nm film): (a) $20 \times 20 \mu \mathrm{m}^{2}$ (b) $5 \times 7.5 \mu \mathrm{m}^{2}$; height scales $150 \mathrm{~nm}$.

The morphology of the fiberlike nanostructures can be seen in more detail by means of atomic force microscopy in Fig 4.20. They are rounded in shape possessing a width of a few hundred nanometer, height up to $200 \mathrm{~nm}$, and length up to $10 \mu \mathrm{m}$. The space between the well separated fibers for high substrate temperatures, $\mathrm{T}_{S}=430 \mathrm{~K}$, is covered by a large number of islands with diameters of several ten nanometers or a few micrometer, respectively. The height of these islands is $2.5 \mathrm{~nm}$ or sometimes $5 \mathrm{~nm}$ for the less expanded islands indicating upright standing molecules on the surface. 


\subsubsection{Di-Nitro- $p$-Quaterphenylene (NOP4)}

The yellow coloured nitro functionalised $p$-quaterphenylene oligomer NOP4 forms well shaped nanostructures upon vapour deposition. For relatively high substrate temperatures $\left(T_{S}=370 \mathrm{~K}\right)$ nanofibers with length of several ten micrometer as well as elongated clusters are visible, oriented along muscovite $\langle 110\rangle$ as usual.
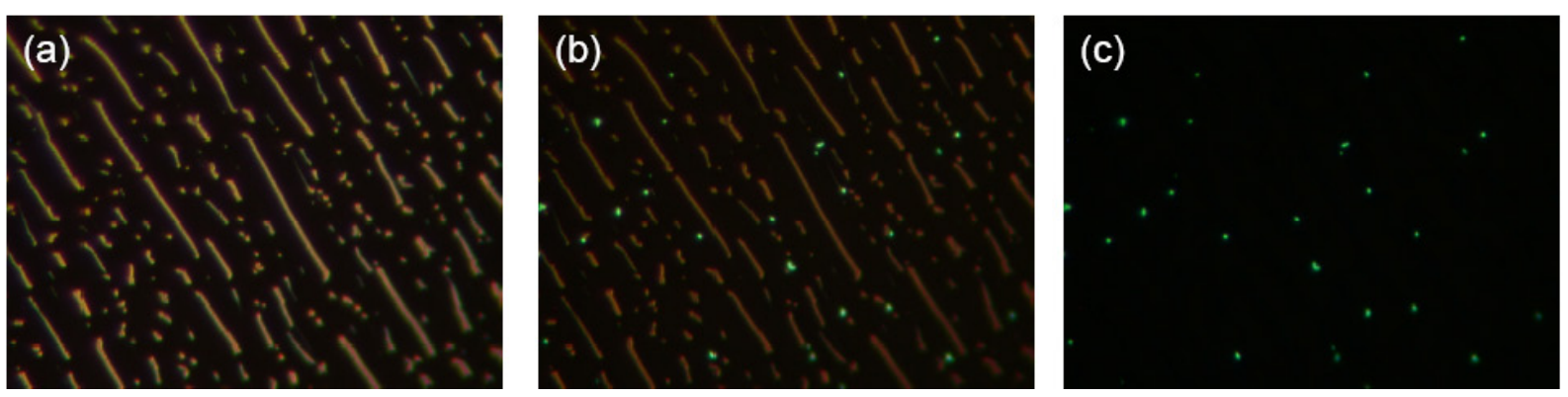

Fig. 4.21: NOP4 on muscovite mica, $T_{S}=370 \mathrm{~K}$, film thickness $10 \mathrm{~nm}$ : (a) darkfield microscopy image, (b) combined darkfield and fluorescene microscopy image, and (c) fluorescence microscopy image; all images $113 \times 85 \mu^{2}$.

The nanostructures are non-fluorescent due to the nitro group. In general, fluorescence of aromatic hydrocarbons possessing a nitro substituent is not detectable [170]. For the nitro group the $n \rightarrow \pi$ transition is the lowest lying. Such transitions have dominant non-radiative de-excitation processes, which lead to low fluorescence efficiency or even quenching. Because of the existence of a low-lying $n \rightarrow \pi$ transition the efficiency for intersytem crossing processes is increased. Therfore many nitro-aromatics, e.g. 2nitronaphthalene, are phosphorescent. But this is not the case for NOP4. Interestingly some small clusters are green fluorescent.
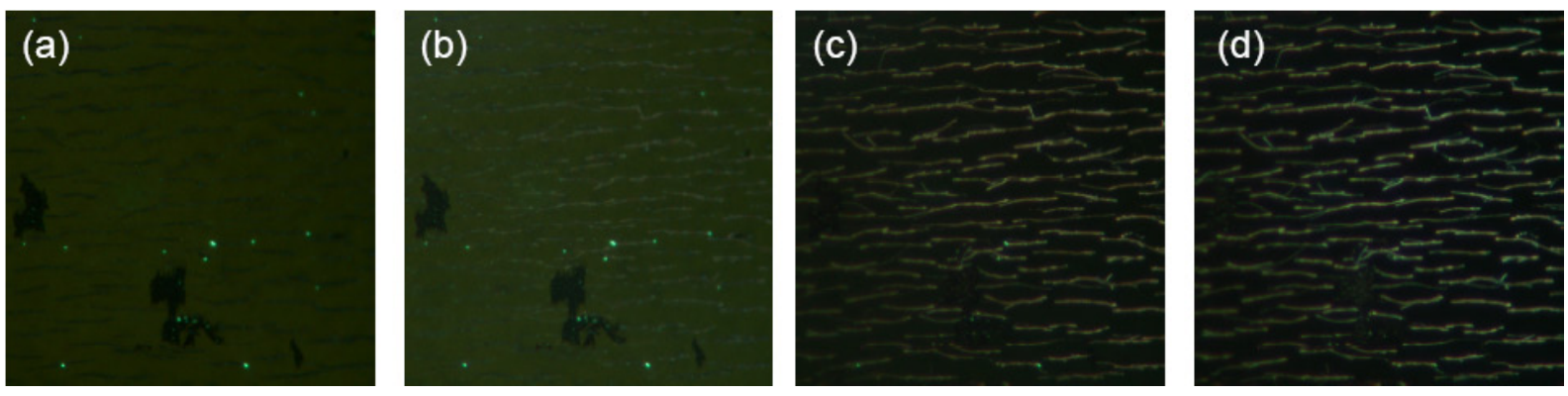

Fig. 4.22: Fluorescence microscopy images of NOP4 on muscovite mica, $T_{S}=330 \mathrm{~K}$, $6 \mathrm{~nm}$ film, of the same area on the sample for increasing illumination times $\left(\lambda_{\text {exc }}=365\right.$ $\mathrm{nm}$ ): (a) $0 \mathrm{sec}$, (b) $20 \mathrm{sec}$, (c) $40 \mathrm{sec}$, and (d) $60 \mathrm{sec}$; all images $95 \times 95 \mu \mathrm{m}^{2}$.

For relatively low substrate temperatures $\left(T_{S}=330 \mathrm{~K}\right)$ nanofibers look irregular and slightly shorter. In between the fibers a few clusters and an almost closed, weakly green 
fluorescent film are visible. Sometimes the film is interrupted by islands of upright standing oligomers on the surface, which appear black in the fluorescence microscopy images (Fig.4.22). Upon irridiation with UV light from a high pressure mercury lamp $\left(\lambda_{\text {exc }}\right.$ $=365 \mathrm{~nm}$ ), the weak green fluorescence of the film and the clusters vanishes within a minute. Only the nanofibers remain weakly yellow fluorescent. This quick bleaching can be due to changes in the chemical constitution of the molecules. It is known that nitroaromatics undergo photodegradation. For instance, 9-nitroanthracene is transformed into anthrachinone upon irradiation [170].

However, even this highly polar nitro substituent is accepted within the growth process leading to fiberlike nanostructures. The fluorescence properties are dominated by the influence of the nitro group. 


\subsection{Mono functionalised para-Quaterphenylenes}

\subsubsection{1-Methoxy-p-Quaterphenylene (MOHP4)}

Since the methoxy group emerged as a well suited functional group in the previous growth studies, MOHP4 was considered as a promising oligomer, too. But the resulting nanoaggregates turned out to be comparatively short and less well aligned, but anyhow they show blue luminescence after excitation with UV-light $\left(\lambda_{\text {exc }} \approx 365 \mathrm{~nm}\right)$.
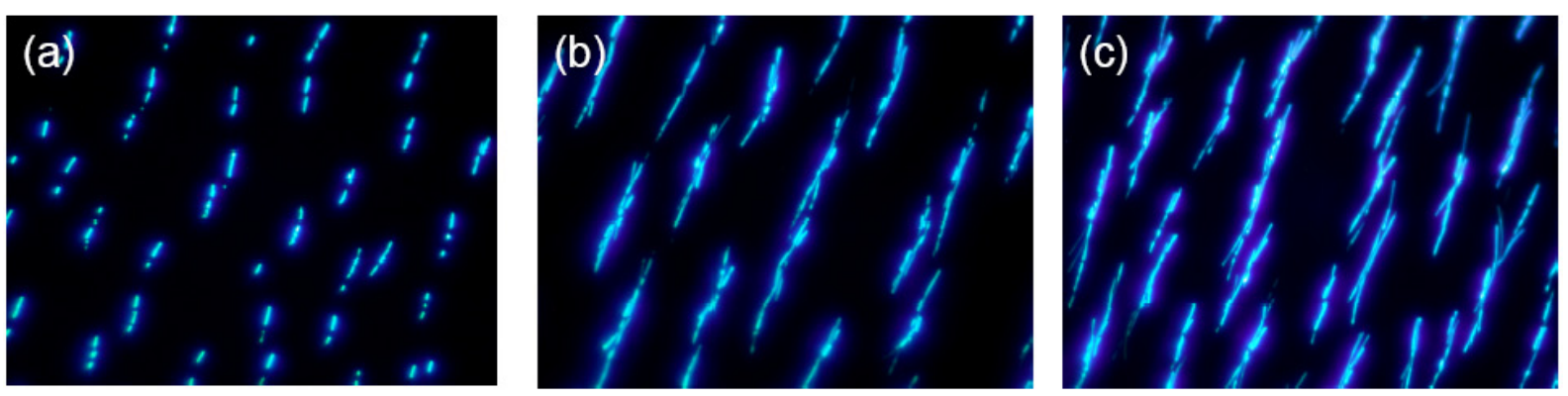

Fig. 4.23: Fluorescence microscopy images $\left(\lambda_{\text {exc }} \approx 365 \mathrm{~nm}\right)$ of MOHP4 on muscovite mica: (a) $113 \times 85 \mu \mathrm{m}^{2}, 2 \mathrm{~nm}$, (b) $80 \times 60 \mu \mathrm{m}^{2}, 4 \mathrm{~nm}$, and (c) $97 \times 73 \mu \mathrm{m}^{2}, 6 \mathrm{~nm}$.

Fig 4.23 shows fluorescence microscopy images for a fixed substrate temperature $\left(T_{S}=\right.$ $340 \mathrm{~K}$ ) and increasing nominal thicknesses of MOHP4: $2 \mathrm{~nm}, 4 \mathrm{~nm}$ and $6 \mathrm{~nm}$. The nanostructures gain length up to a few ten micrometer, Fig.4.24(a), and width for increasing coverages. The aggregate number density does not change significantly, Fig.4.24(b).
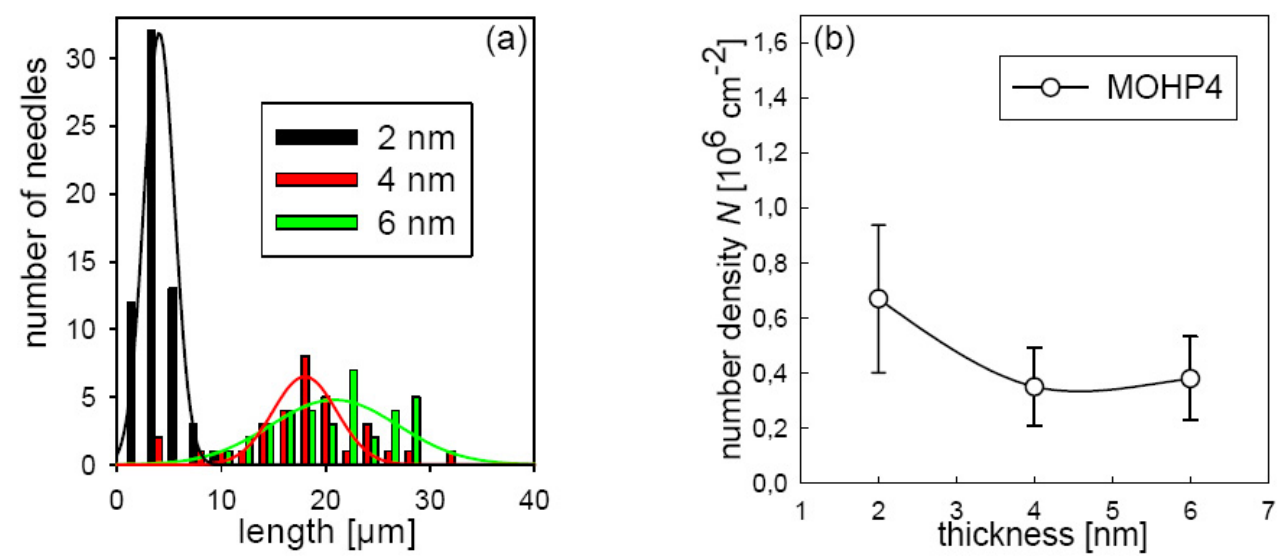

Fig. 4.24: (a) Length distribution and (b) aggregate number density for deposition at $T_{S}=340 \mathrm{~K}$ and for different film thicknesses of MOHP4. Solid lines in (a) Gaussian fits, in (b) to guide the eye.

As far as one can conclude from the fluorescence microscopy images, the nanofibers exhibit an unsteady shape. AFM-images, Fig 4.25 show that the structures are built up of smaller elongated aggregates, which assemble to form a fiberlike structure, the nano-lego 
phenomenon. Sometimes nano-branches are visible similar to the low surface temperature case for the MOCLP4 (see next section).
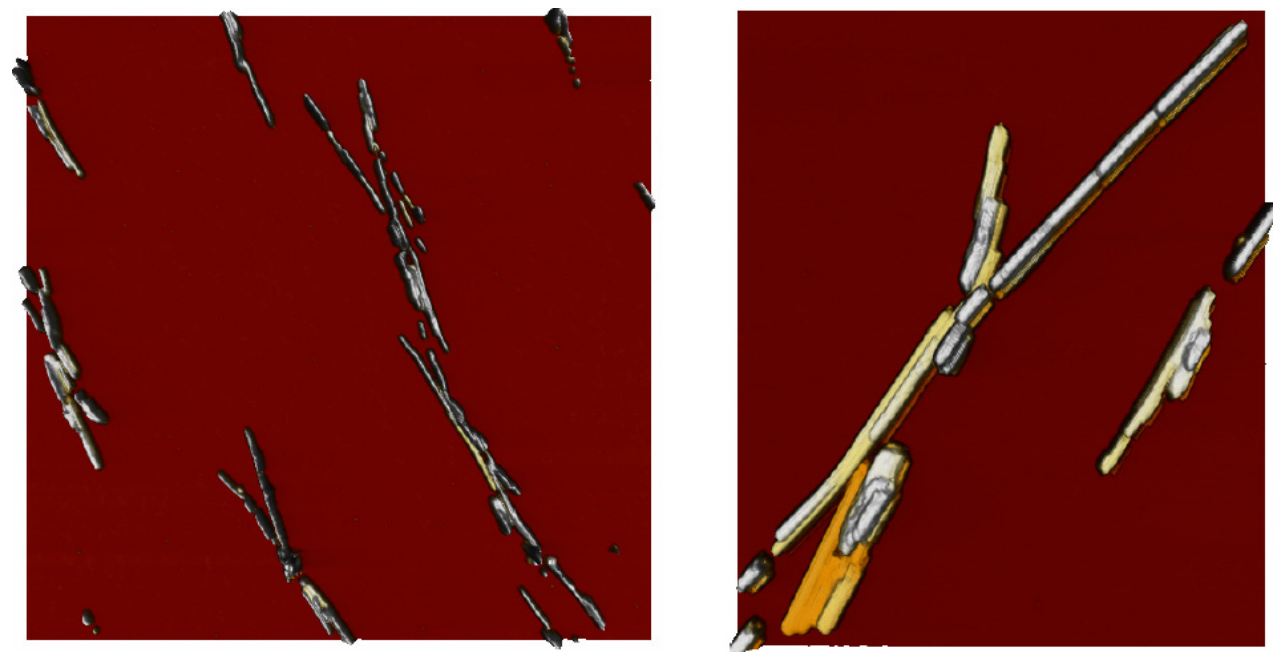

Fig. 4.25: AFM-images of MOHP4 on muscovite mica, $T_{S}=340 \mathrm{~K}$, nominal film thickness $6 \mathrm{~nm}$. left: $35 \times 35 \mu \mathrm{m}^{2}$, height scale $118 \mathrm{~nm}$, right: $12 \times 15 \mu \mathrm{m}^{2}$, height scale 155 $\mathrm{nm}$.

\subsubsection{1-Chloro-p-Quaterphenylene (CLHP4)}

Next CLHP4 has been vapour deposited, while the substrate temperature was kept at $360 \mathrm{~K}$.
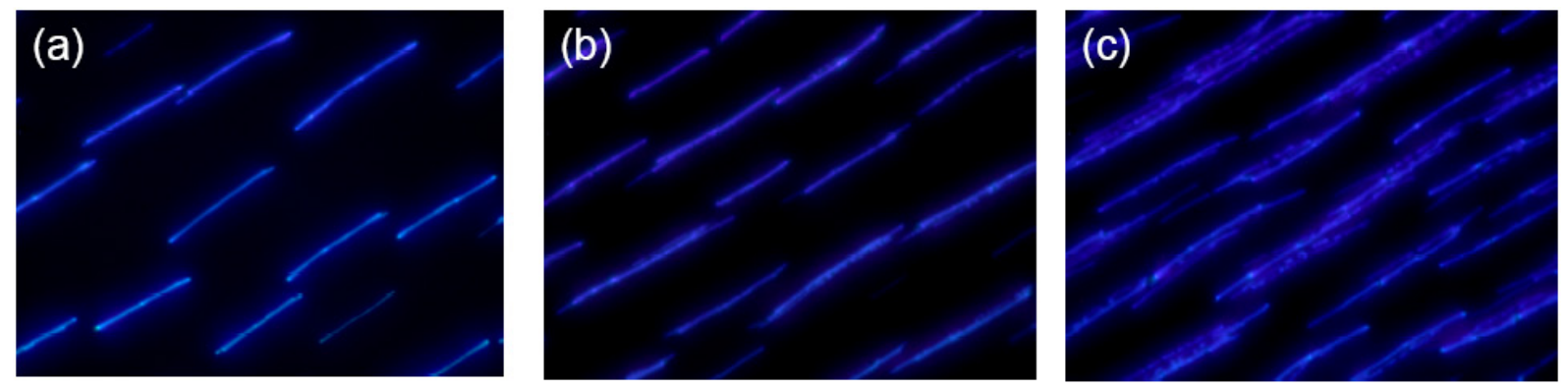

Fig. 4.26: Fluorescence microscopy images $\left(\lambda_{e x c} \approx 365 \mathrm{~nm}\right)$ of CLHP 4 on muscovite mica for different nominal film thicknesses $\left(T_{S}=360 \mathrm{~K}\right)$ : (a) $60 \times 45 \mu \mathrm{m}^{2}, 4 \mathrm{~nm}$, (b) 60 $\times 45 \mu \mathrm{m}^{2}, 8 \mathrm{~nm}$, and (c) $56 \times 42 \mu \mathrm{m}^{2}, 12 \mathrm{~nm}$.

The fluorescence microscopy images, Fig 4.26, show the resulting parallel aligned nanofibers for increasing nominal film thicknesses of CLHP4: $4 \mathrm{~nm}, 8 \mathrm{~nm}$ and $12 \mathrm{~nm}$. The length of the fibers is about the same for all thicknesses namely a few ten micrometer, Fig. 4.27(a). The aggregate number density $N$ is about the same for all film thicknesses, too, Fig 4.27(b), whereas in case of the symmetrically functionalised oligomer with two 
chloride groups for the same substrate temperature $\left(\mathrm{T}_{S}=360 \mathrm{~K}\right) N$ increases significantly and saturates for high coverages.
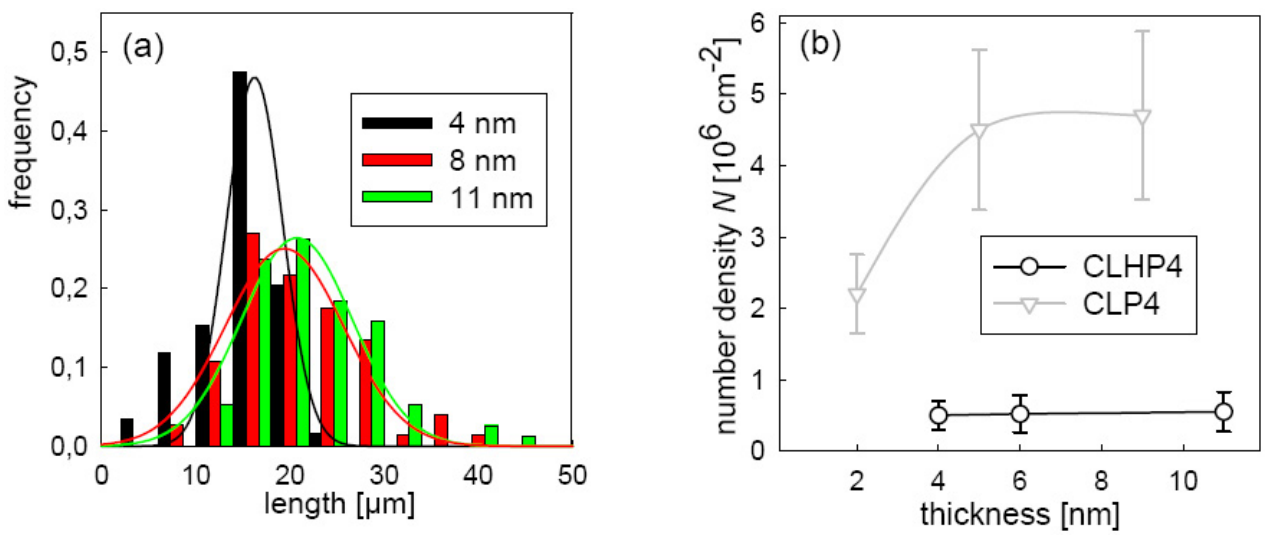

Fig. 4.27: (a) Length distribution and (b) aggregate number density for deposition at $T_{S}=340 \mathrm{~K}$ and for different film thicknesses of CLHP4. Solid lines in (a) Gaussian fits, in (b) to guide the eye.

The width of the CLHP4 nanofibers increases with increasing coverage and the well separated fibers get more densely packed. Widening of the fibers associates with getting more inhomogeneous in shape. This can be seen in more detail by means of atomic force microscopy (Fig 4.28, Fig.4.29).
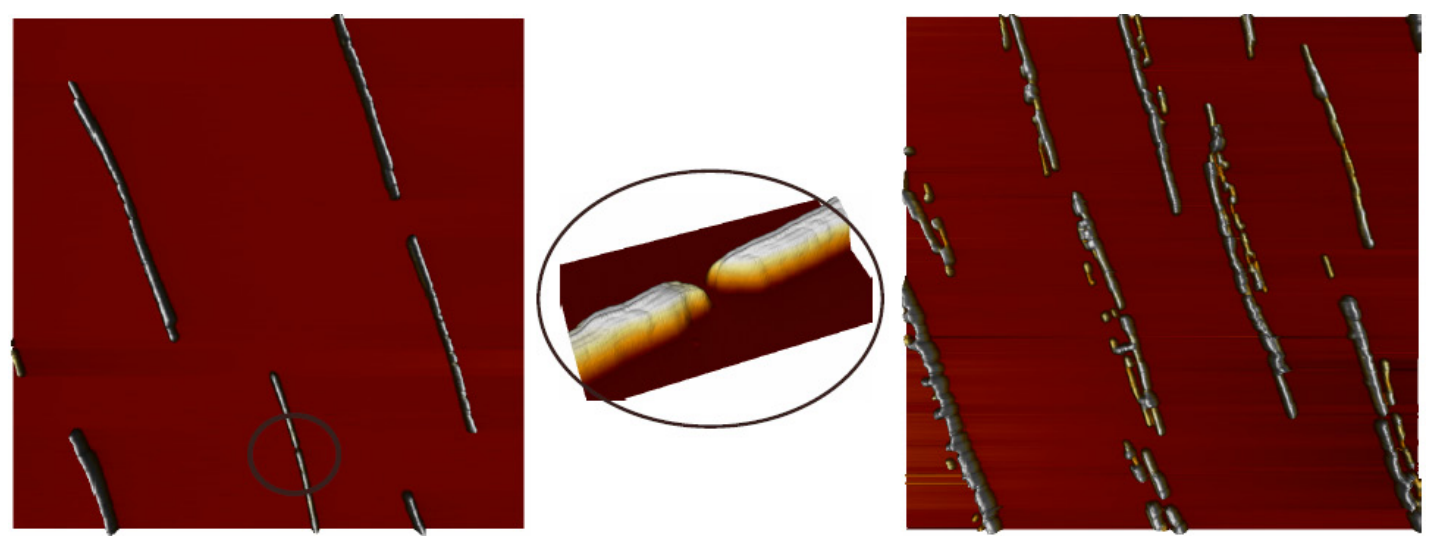

Fig. 4.28: AFM-images of CLHP4 on muscovite mica, substrate temperature $T_{S}=360$ K. left: $30 \times 30 \mu \mathrm{m}^{2}$, height scale $110 \mathrm{~nm}$, nominal thickness $4 \mathrm{~nm}$; enlarged extract: $3 \times$ $1.7 \mu \mathrm{m}^{2}$, height scale $160 \mathrm{~nm}$; right: $30 \times 30 \mu \mathrm{m}^{2}$, height scale $200 \mathrm{~nm}$, nominal thickness $12 \mathrm{~nm}$.

For low coverages (film thickness $4 \mathrm{~nm}$ ) the fibers are well separated, parallel aligned and rounded in shape. The height is about $100 \mathrm{~nm}$ and width about $600 \mathrm{~nm}$. Sometimes breaks within a fiber are visible, which appear as bright spots in the fluorescence microscopy images. As for p6P the fibers act as distinct waveguides [46-48] and the light is scattered at breaks within the fiber. 

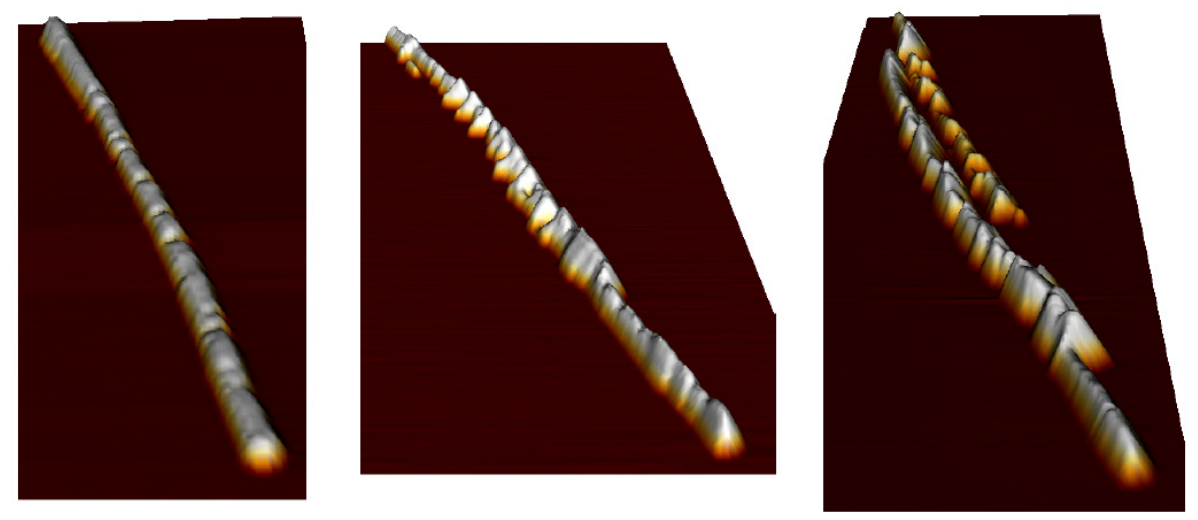

Fig. 4.29: AFM-images of single fibers from CLHP4 on muscovite mica $\left(T_{S}=360 \mathrm{~K}\right)$ for increasing nominal film thicknesses: left: height scale $110 \mathrm{~nm}, 4 \mathrm{~nm}$ (fiber length 12 $\mu \mathrm{m})$; middle: height scale $160 \mathrm{~nm}, 8 \mathrm{~nm}$ (fiber length $22 \mu \mathrm{m}$ ); right: height scale $210 \mathrm{~nm}$, $12 \mathrm{~nm}$ (fiber length $18 \mu \mathrm{m}$ ).

For higher coverages (nominal thickness $8 \mathrm{~nm}$ ) the fibers gain height and width and become more peaked and irregular reminding one of carnivore teeth. The height is now up to $250 \mathrm{~nm}$ and the width up to $1 \mu \mathrm{m}$. Going to higher coverages (film thickness 12 $\mathrm{nm}$ ) does not increase height and width of a single fiber, but new fibers grow in very close distance (few nanometer) to the former fibers. Under the fluorescence microscope this looks like kind of unsteady broadening of the nanoaggregates. This can also be called nano-lego phenomenon similar to MOHP4 (see above), but the CLHP4 aggregates are superiorly shaped and aligned.

Unfortunately, no temperature dependence of the nanostructures's shape have been studied, yet. This will be part of future experiments, since the oligomers functionalised with chloride groups - CLP4 see section above, and MOCLP4 see section below - assemble to morphologically different structures strongly depending on the growth temperature.

\subsubsection{1-N,N-Dimethyl-Amino- $p$-Quaterphenylene (NMeHP4)}

NMeHP4 has been deposited on a freshly cleaved as well as on a non-cleaved muscovite mica substrate. The substrate temperature was kept at $340 \mathrm{~K}$ during deposition for both samples. Fig 4.30 shows fluorescence microscopy images of NMeHP4 on the freshly cleaved muscovite mica sample for three different nominal thicknesses: $2 \mathrm{~nm}, 4 \mathrm{~nm}$ and $6 \mathrm{~nm}$.

The fiberlike nanostructures are slightly bent (wormlike) but still mutually aligned, similar to the di-functionalised oligomer NMeP4. The length of the nanofibers increases with increasing coverages from several micrometer up to a few ten micrometer, Fig.4.31(a). The aggregate number density is similar for the three coverages at $\mathrm{T}_{S}=340 \mathrm{~K}$, Fig, 4.31 (b).

The nanofibers taper off at the endings. As far as one can conclude out of the increase of fluorescence intensity the fibers gain in height as well. This is proven by atomic force 

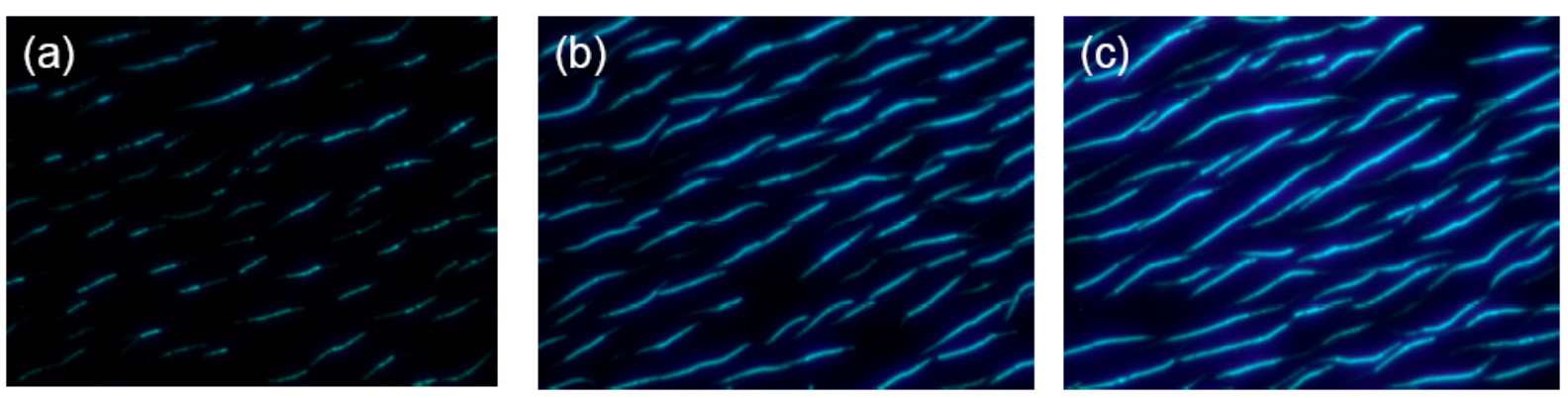

Fig. 4.30: Fluorescence microscopy images $\left(\lambda_{\text {exc }} \approx 365 \mathrm{~nm}\right)$ of NMeHP4 on muscovite mica (freshly cleaved), $T_{S}=340 \mathrm{~K}$ nominal film thicknesses: (a) $2 \mathrm{~nm}$, (b) $4 \mathrm{~nm}$, and (c) $6 \mathrm{~nm}$; image sizes $56 \times 42 \mu \mathrm{m}^{2}$.
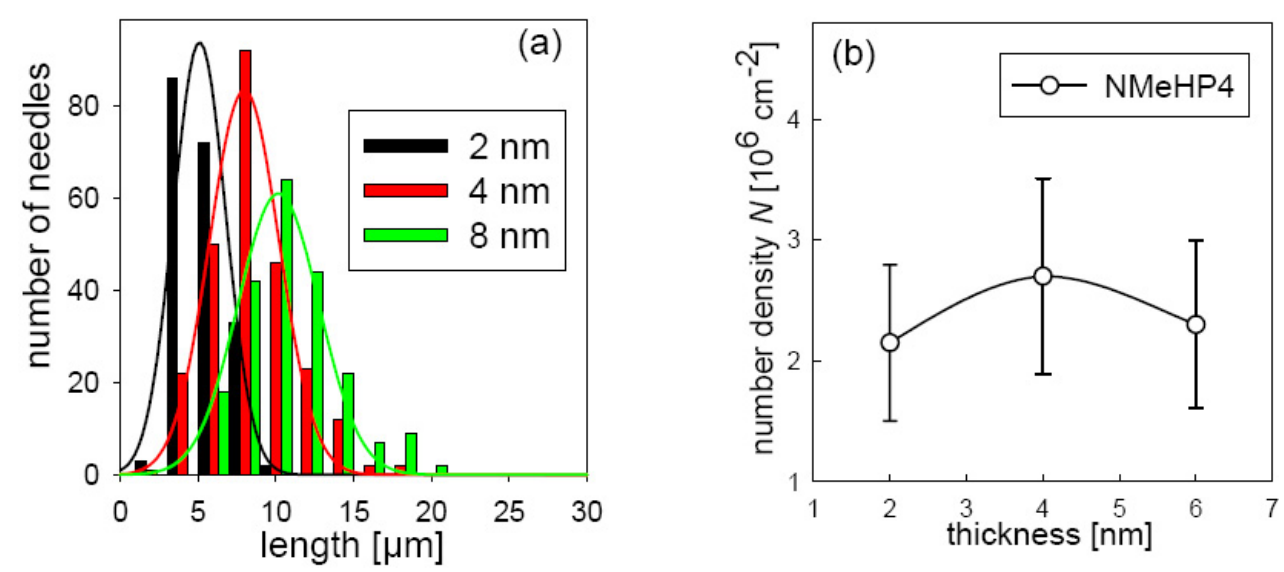

Fig. 4.31: (a) Length distribution and (b) aggregate number density for deposition at $T_{S}=340 \mathrm{~K}$ and for different film thicknesses of NMeHP4. Solid lines in (a) Gaussian fits, in (b) to guide the eye.

microscopy (Fig, 4.32): For low coverage (nominal thickness $2 \mathrm{~nm}$ ) the nanofibers possess heights and widths of about $80 \mathrm{~nm}$ and $400 \mathrm{~nm}$, respectively, and they increase in height to about $160 \mathrm{~nm}$ and in width to $500 \mathrm{~nm}$ for higher coverage (nominal thickness $6 \mathrm{~nm}$ ). The shape of the cross-section is rounded.

The importance of using a freshly cleaved muscovite mica substrate for generating nanofibers can be deduced from Fig.4.33. If NMeHP4 is deposited on a non-cleaved muscovite mica substrate the nanostructures look different. Only a freshly cleaved muscovite mica substrate has a polar, hydrophilic surface with a high surface energy [171]. For older substrates the surface changes to hydrophobic and looses surface energy. Deposition of p6P onto a water-treated muscovite mica substrate leads to formation of microrings instead of nanofibers and to layers of upright standing molecules [172]. Similar to those micro-rings, NMeHP4 forms bent nanostructures on the non-cleaved substrate, Fig 4.33(a), and additionally poorly fluorescent islands can be seen in the background. In other regions on the sample mutually aligned fluorescent fibers are visible interrupted 

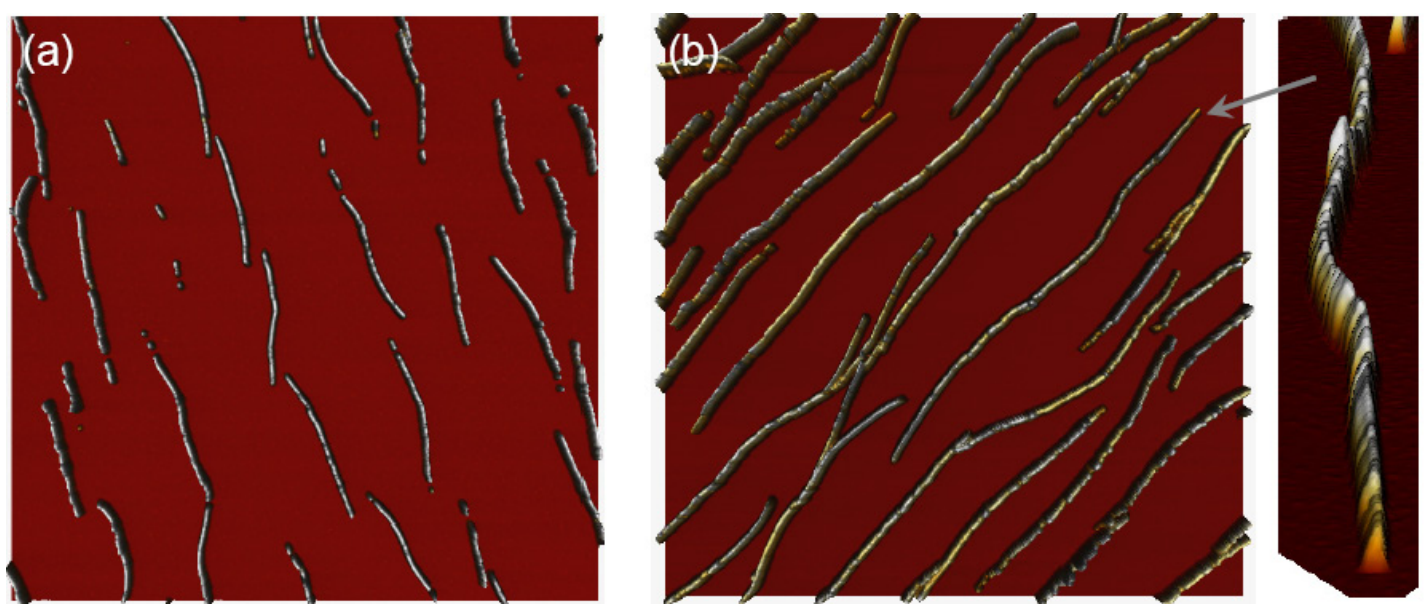

Fig. 4.32: AFM-images of NMeHP4 on muscovite mica (freshly cleaved) substrate temperature $340 \mathrm{~K}$ (a) $24 \times 24 \mu \mathrm{m}^{2}$, height scale $72 \mathrm{~nm}$, film thickness $2 \mathrm{~nm}$; (b) $23 \times 23 \mu \mathrm{m}^{2}$, height scale $204 \mathrm{~nm}$, film thickness $6 \mathrm{~nm}$. On the right hand side an enlarged 3D-extract of the indicated fiber is shown.

by black areas. These stem from upright standing molecules on the surface, which show no fluorescence under normal incidence. It has been reported for $\mathrm{p} 6 \mathrm{P}$ on other inorganic substrates, that defects or contaminations lead to the thermodynamically more stable phase with upright standing molecules on the surface [173]. This has to be verified here by means of atomic force microscopy in the future.
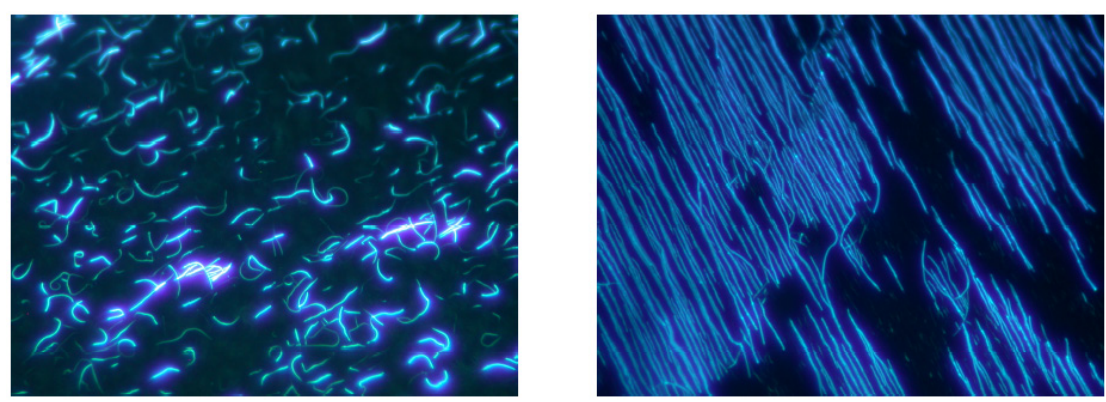

Fig. 4.33: Fluorescence microscopy images $\left(\lambda_{\text {exc }} \approx 365 \mathrm{~nm}\right)$ of NMeHP4 on muscovite mica (non-cleaved), $T_{S}=340 \mathrm{~K}$; nominal thicknesses: $8 \mathrm{~nm}$; left: $160 \times 120 \mu \mathrm{m}^{2}$, right: $110 \times 82 \mu \mathrm{m}^{2}$.

\subsubsection{1-Cyano- $p$-Quaterphenylene (CNHP4)}

Last but not least among the mono functionalised oligomers CNHP4 has been deposited on a freshly cleaved muscovite mica substrate at elevated substrate temperatures. Since MOHP4 shows clearly inferior fiber growth compared to the prominent di-functionalised oligomer MOP4, it is obvious, that not only the functional group itself decides on improved 
growth but also the counterpart functionality has to be taken in account. In this case of the cyano group the CNHP4 shows superior growth properties compared to the difunctionalised CNP4.
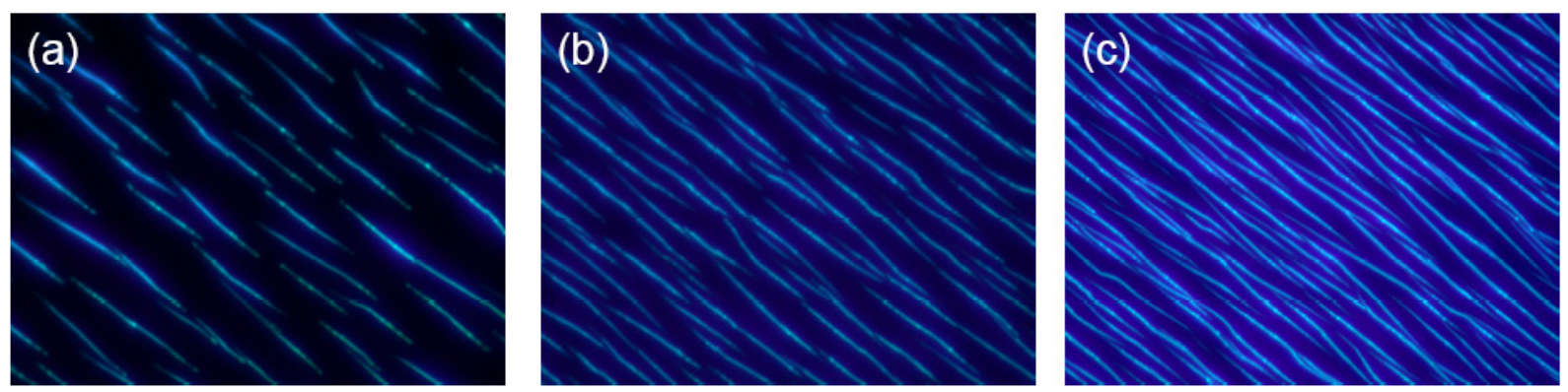

Fig. 4.34: Fluorescence microscopy images of CNHP4 on muscovite mica for increasing film thicknesses $\left(T_{S}=340 \mathrm{~K}\right)$ : (a) $56 \times 42 \mu \mathrm{m}^{2}$, film thickness $4 \mathrm{~nm}$; (b) $56 \times 42 \mu \mathrm{m}^{2}$, film thickness $8 \mathrm{~nm}$; (c) $88 \times 65 \mu \mathrm{m}^{2}$, film thickness $12 \mathrm{~nm}$.
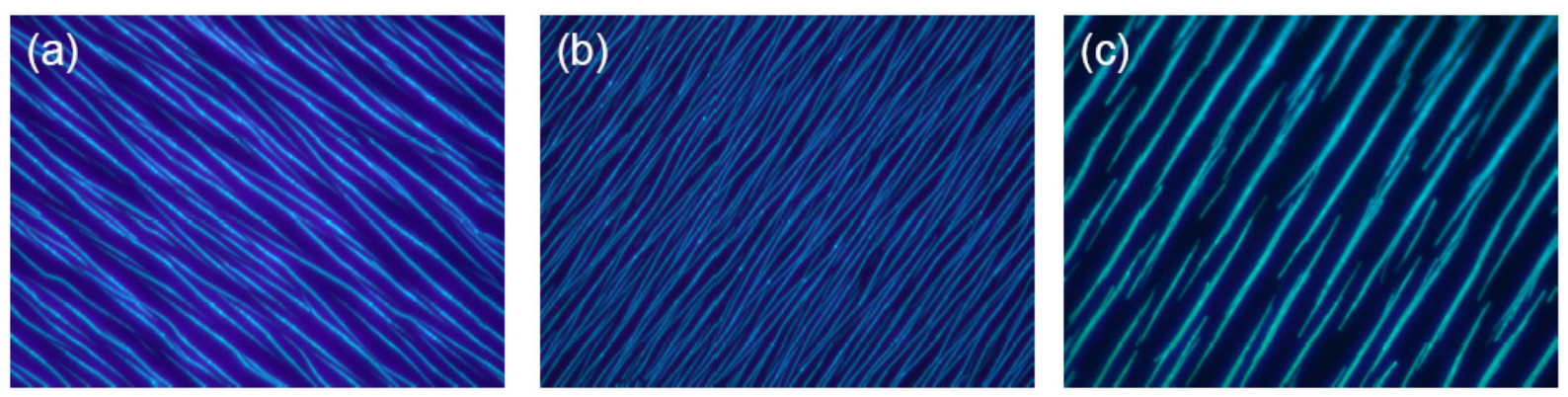

Fig. 4.35: Fluorescence microscopy images of CNHP4 on muscovite mica for different substrate temperatures, film thicknesses $12 \mathrm{~nm}$ : (a) $88 \times 65 \mu m^{2}, T_{S}=340 \mathrm{~K}$; (b) 113 $\times 85 \mu \mathrm{m}^{2}, T_{S}=360 \mathrm{~K} ;(\mathrm{c}) 83 \times 63 \mu \mathrm{m}^{2}, T_{S}=380 \mathrm{~K}$.

The resulting parallel aligned CNHP4 nanofibers are shown in fluorescence microscopy images, Fig 4.34 for increasing nominal film thicknesses: $4 \mathrm{~nm}, 8 \mathrm{~nm}$ and $12 \mathrm{~nm}$, deposited at $\mathrm{T}_{S}=340 \mathrm{~K}$. For increasing coverages the fibers clearly gain in length starting from a few ten micrometer up to several ten micrometer, Fig 4.37(a). Initially short aggregates form with certain height and width, and additionally deposited organic material augments the number of aggregates. For further deposition of material the dominating effect is an increase in length of the fibers, which is indicated by the peaked course of the aggregate number density, Fig 4.37(b).

For generating long, mutually aligned nanofibers not only an appropriate amount of organic material is needed but also an optimal substrate temperature. Fig.4.35 shows fluorescence microscopy images of CNHP4 on muscovite mica for different substrate temperatures and similar nominal film thicknesses, namely $12 \mathrm{~nm}$. Obviously the growth conditions comprising a film thickness of $12 \mathrm{~nm}$ and a substrate temperature of $360 \mathrm{~K}$ are 

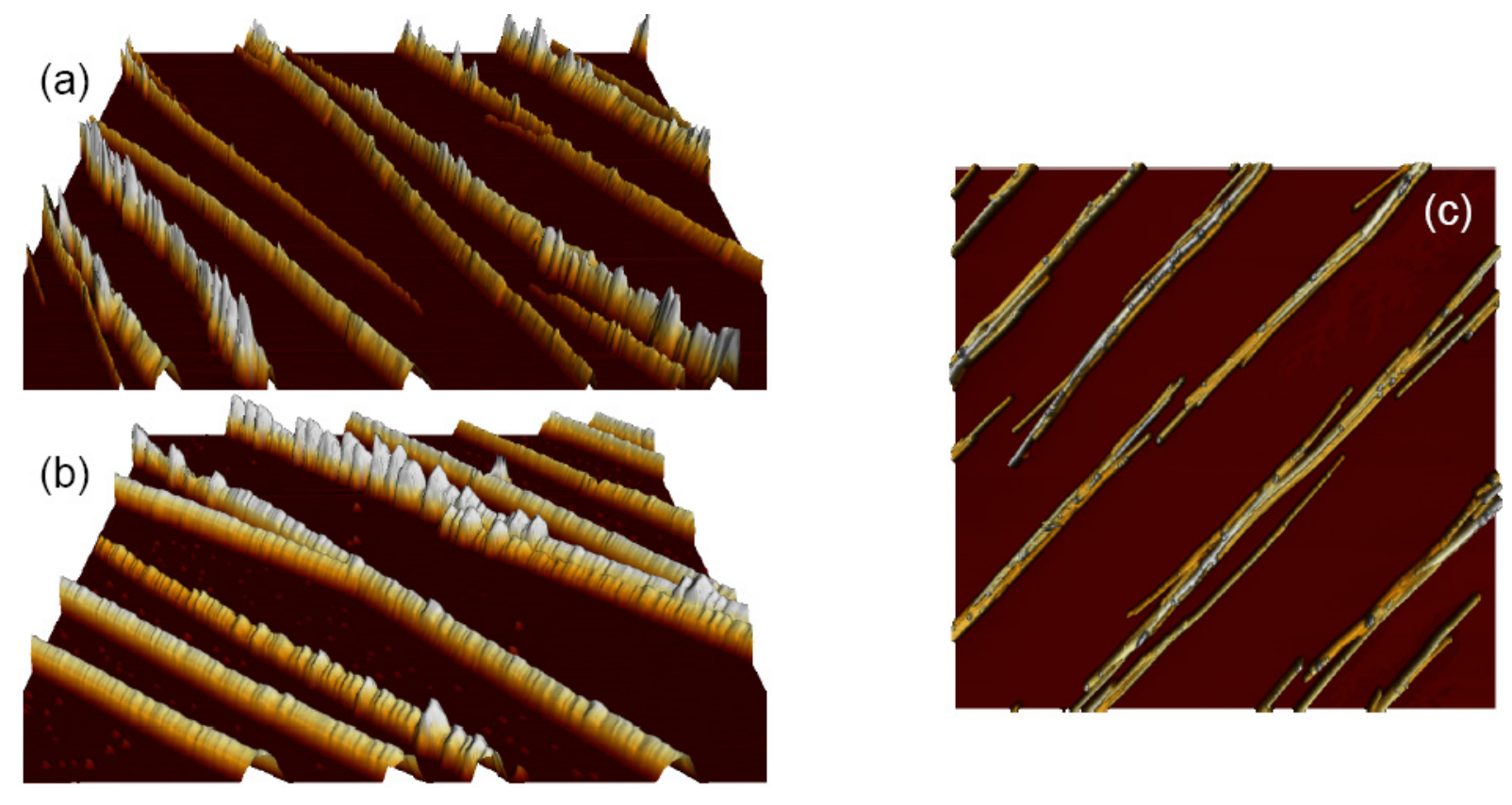

Fig. 4.36: AFM images of CNHP4 on muscovite mica for different substrate temperatures, nominal film thicknesses $12 \mathrm{~nm}$ : (a) $T_{S}=340 \mathrm{~K}, 15 \times 15 \mu \mathrm{m}^{2}$; (b) $T_{S}=360 \mathrm{~K}, 25$ $\times 25 \mu \mathrm{m}^{2}$, height scales $300 \mathrm{~nm}$ both; (c) $T_{S}=380 \mathrm{~K}, 30 \times 30 \mu \mathrm{m}^{2}$, height scale $80 \mathrm{~nm}$.

the most favourable among the given samples for generating nanofibers with length up to several hundred micrometers (Fig 4.35(b)).

The fibers are mutually aligned but not strictly parallel. Within each domain three growth direction can be observed: along muscovite $\langle 110\rangle$ and along $\langle 110\rangle \pm 7^{\circ}$ with an experimental error of $\pm 3^{\circ}$. The nanofibers branch out forming kinks and switch between growth directions.

The morphology of the nanoaggregates can be seen in more detail in AFM images. The nanofibers look like an earthworm with combat-crown. For substrate temperature $T_{S}=$ $340 \mathrm{~K}$ (Fig 4.36(a)) the fibers grow to a height of about $200 \mathrm{~nm}$ and a width of about $500 \mathrm{~nm}$, then they increase in length up to several ten micrometers. The fibers taper off at the endings and elongate in this way. For high coverages (12 nm film thickness) the nanofibers reach with their peaked top parts heights of about $300 \mathrm{~nm}$ or even $400 \mathrm{~nm}$. These peaks are visible in the fluorescence microscopy images as bright spots on top of the nanofibers. This bright spots can be attributed to increased fluorescence intensity due to more material within the peaks. According to this they differ from the bright spots e.g. in case of CLHP4 (see above), which originate from scattered light at breaks within the fiber.

For a slightly higher substrate temperature, $T_{S}=360 \mathrm{~K}$ (Fig 4.36(b)), the fibers become bigger without changing their shape: They exhibit a mean width of $700 \mathrm{~nm}$ and a mean height of $250 \mathrm{~nm}$, including peaks the height is even up to $500 \mathrm{~nm}$. The length increases 

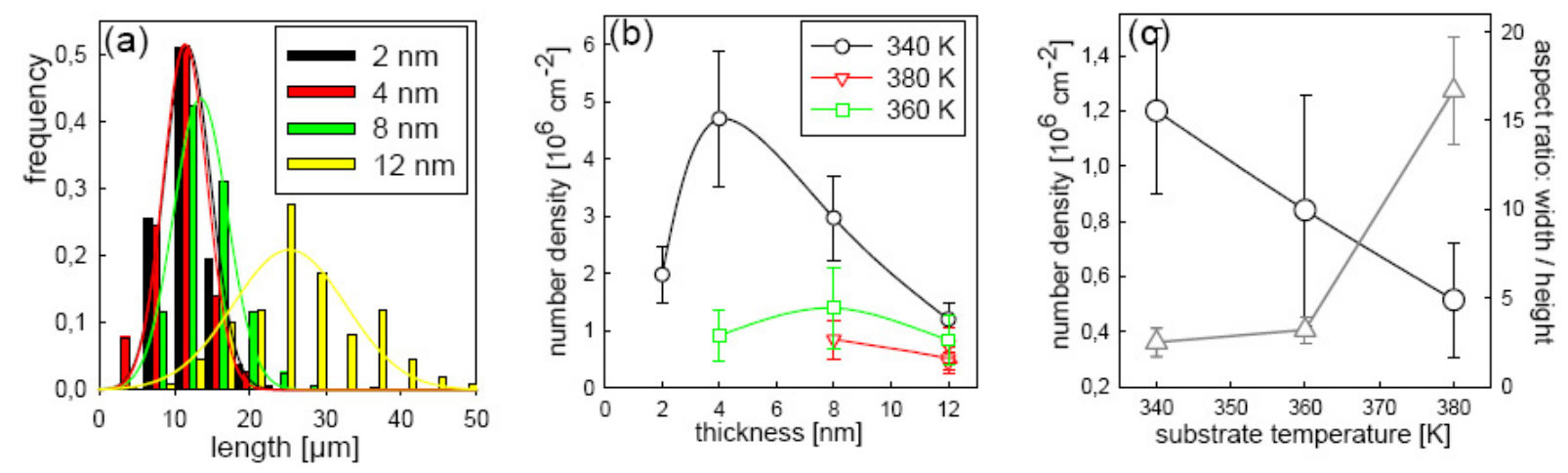

Fig. 4.37: (a) Length distribution for deposition at $T_{S}=360 \mathrm{~K}$, (b) aggregate number density for different substrate temperatures and film thicknesses, and (c) aggregate number density (circles) and aspect ratio: width/height (triangles) for different substrate temperatures and for $12 \mathrm{~nm}$ film thickness of CNHP4. Solid lines in (a) Gaussian fits, in (b) and (c) to guide the eye.

to a several hundred micrometers. Small clusters of a few ten nanometer are visible in between the needles, which are believed to be needle precursors [66, 67]. For a further increase in substrate temperature, $T_{S}=380 \mathrm{~K}$ (Fig 4.36(c)), the fibers possess a modified shape and aspect ratio. The fibers are wider and flatter with a mean height around 60 $\mathrm{nm}$ and width up to $1 \mu \mathrm{m}$, length is decreased to several ten micrometers. The aggregates look fibrous and frayed and have a larger depletion zone compared to lower substrate temperatures. This reflects a higher surface mobility of the aggregates. Expanded flat and dendritic islands are visible in between the fibers with a height of $2.0 \mathrm{~nm}$ indicating upright standing molecules on the surface.

Aggregate number densities and aspect ratios for the different substrate temperatures just discussed and a constant film thickness $(12 \mathrm{~nm})$ are shown for comparison in Fig. 4.37(c).

Compared to aggregates from the difunctionalised CNP4 the mono functionalised CNHP4 exhibits superior properties for generating nanofibers. This may be attributed to enhanced intermolecular interactions, which lead to improved packing of the molecules within the nanofibers. It can be concluded that not only the functional group itself determines the growth behaviour but also the counterpart functionalisation has to be taken into account, leading to adjusted possibilities for intermolecular interactions. Seeing that only two positions are considered to be functionalised at all, demonstrates the complexity of effects caused by functionalisation. 


\subsection{Differently functionalised para-Quaterphenylenes}

\subsubsection{Methoxy-Amino-p-Quaterphenylene (MONHP4) [6]}

The non-symmetrically substituted $p$-quaterphenylene with a methoxy group and an amino group MONHP4 has been vapour deposited onto a freshly cleaved muscovite mica substrate. This results in well aligned fiberlike nanostructures, which are non-fluorescent in a fluorescence microscope due to intermolecular hydrogen bondings between amino and methoxy groups (cf. NHP4 and see Chapter Linear Optics for details).
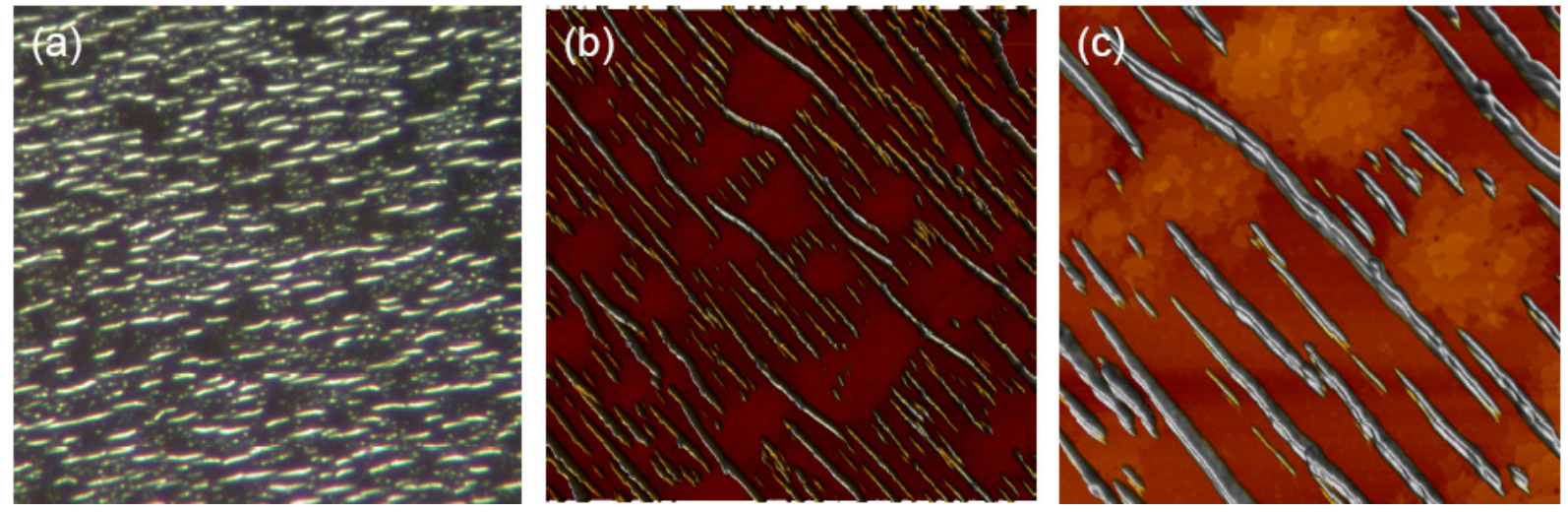

Fig. 4.38: MONHP4 on muscovite mica, $T_{S}=380 \mathrm{~K}$, film thickness $7 \mathrm{~nm}$, (a) darkfield microscopy images $85 \times 85 \mu \mathrm{m}^{2}$, and atomic force microscopy images (b) $15 \times 15 \mu \mathrm{m}^{2}$, height scale $130 \mathrm{~nm},\left(\right.$ c) $5 \times 5 \mu \mathrm{m}^{2}$, height scale $50 \mathrm{~nm}$.
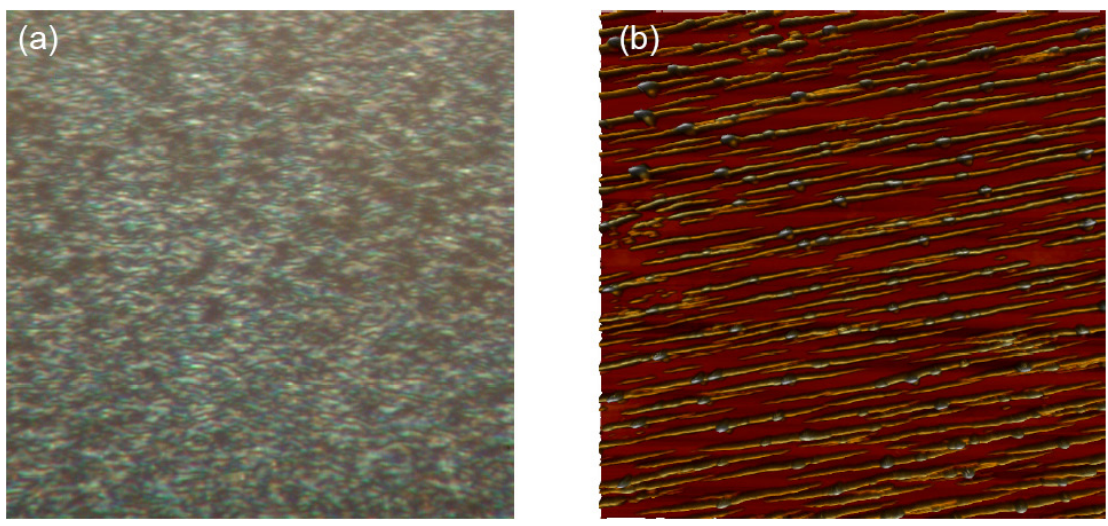

Fig. 4.39: MONHP4 on muscovite mica, $T_{S}=360 \mathrm{~K}$, film thickness $7 \mathrm{~nm}$, (a) darkfield microscopy images $85 \times 85 \mu \mathrm{m}^{2}$, and (b) atomic force microscopy images $8 \times 8 \mu \mathrm{m}^{2}$, height scale $130 \mathrm{~nm}$.

The nanofibers possess typical widths of a few hundred nanometer, heights up to 120 $\mathrm{nm}$, and they reach several micrometer in length for $T_{S}=380 \mathrm{~K}$, Fig. 4.38 . In between those fibers elongated clusters are visible as well as islands, whose step heights of $2.5 \mathrm{~nm}$ 
indicate upright standing molecules on the surface (Fig, $4.38(\mathrm{c}))$. Fibers taper off at the endings.

This is more pronounced for a slightly lower substrate temperature, $\mathrm{T}_{S}=360 \mathrm{~K}$, Fig 4.39(b): The fibers possess something like a knob with a height up to $120 \mathrm{~nm}$ in the middle, and they run out at the endings reaching lengths of only a few micrometer. The nanofibers are very close to each other, they are packed too densely to obtain a well resolved darkfield microscopy image, Fig.4.39(a).

\subsubsection{Methoxy-Chloro-p-Quaterphenylene (MOCLP4) [6]}

Vapour deposition of MOCLP4 results in well defined nanostructures, which show a strong dependence on the growth temperature: clearly a high surface temperature case and a low surface temperature case can be distinguished. Thus it seems that this strong morphologically temperature dependence is an intrinsic property of functionalisation with chloride groups for $p$-quaterphenylenes. The possibilty to grow different structures from a single molecule just by changing the substrate temperature adds another degree of freedom for the design of nanoscaled structures.
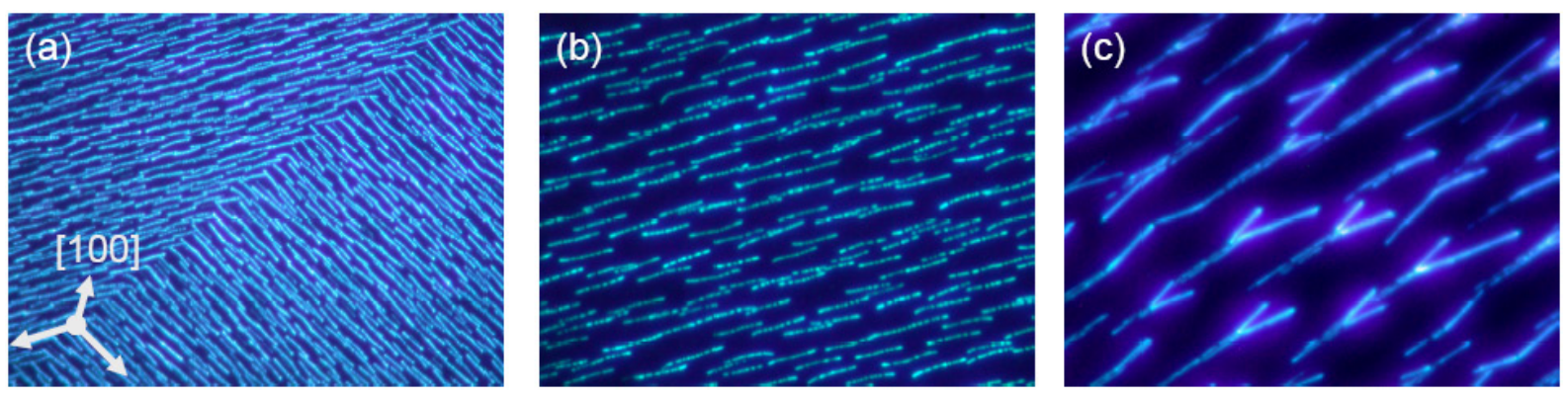

Fig. 4.40: Fluorescence microscopy images of MOCLP4 on muscovite mica (a) $135 \times$ $100 \mu \mathrm{m}^{2}$, (b) $56 \times 42 \mu \mathrm{m}^{2}$, both $T_{S}=400 \mathrm{~K}$ high surface temperature case, film thickness $10 \mathrm{~nm}$, and (c) $56 \times 42 \mu \mathrm{m}^{2}, T_{S}=360 \mathrm{~K}$ low surface temperature case, film thickness 4 $\mathrm{nm}$.

If the substrate temperature is kept at $400 \mathrm{~K}$ (high surface temperature case) mutually aligned fibers are formed possessing lengths of several micrometers or sometimes several ten micrometer, widths of a few hundred nanometer and heights of a few ten nanometer. Width and height are typical for para-phenylene nanofibers, whereas the length is more individual. Here the length is comparable to the high temperature CLP4 fibers. The aggregates from MOCLP4 always grow along the $\langle 110\rangle$ muscovite direction and form two different domains within a single sample, which are rotated by $120^{\circ}$ to each other such as usual (Fig $4.40(\mathrm{a})$ ).

More details of the morphology were obtained by means of atomic force microscopy (Fig 4.41(a)). As deduced from those morphologically well resolved studies, a single fiber 
is made up of short, elongated aggregates, which are well assembled in an alignment to form a fiber. In between the fibers small clusters are visible which cannot be seen in a light microscope. Since they are believed to be needle precursors [66, 67] they reflect the bottom-up fashion of the growth process. The fibers form kinks with an angle of about $15^{\circ}$, which have already been observed in the case of MOP4, the symmetrically functionalised oligomer with two methoxy groups. Thus it seems that the trend to form kinks is an intrinsic property due to the substitution with methoxy groups.
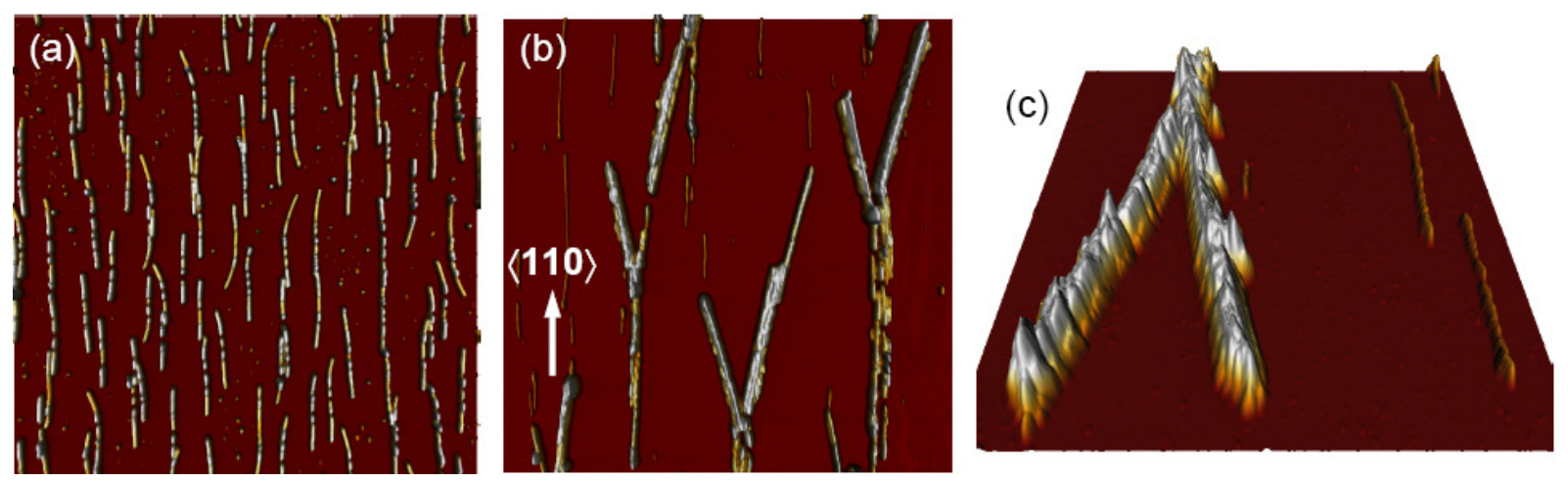

Fig. 4.41: Atomic force microscopy images of MOCLP4 on muscovite mica (a) $25 \times 25$ $\mu \mathrm{m}^{2}$, height scale $80 \mathrm{~nm}, T_{S}=400 \mathrm{~K}$ high surface temperature case, film thickness $10 \mathrm{~nm}$, (b) $18 \times 18 \mu \mathrm{m}^{2}$, and (c) $8 \times 6.5 \mu \mathrm{m}^{2}$, both height scales $130 \mathrm{~nm}$ and $T_{S}=360 \mathrm{~K}$ low surface temperature case, film thickness $4 \mathrm{~nm}$.

Keeping the subtrate temperature at $360 \mathrm{~K}$ during deposition (low surface temperature case) leads to mutually aligned nanobranches. The growth direction is again the $\langle 110\rangle$ muscovite direction before the fiber splits. Then the $\langle 110\rangle$ muscovite direction is the bisecting line of the angle between the branches, which is about $30^{\circ}$ with an experimental error of $\pm 3^{\circ}$. But they exhibit no bilateral symmetry, for most nanobranches one branch is significantly longer, sometimes more than double as long. Similar nanobranches have been found in case of the mono functionalised oligomer with a methoxy group MOHP4. The nanobranches possess overall lengths up to $30 \mu \mathrm{m}$, and with heights up to $150 \mathrm{~nm}$ they are almost double as high as the nanofibers for the high temperature case. In between the nanobranches elongated clusters are located, which cannot be seen in a light microscope, Fig 4.41 (c). Strikingly these grow strictly along muscovite $\langle 110\rangle$, so that they only reflect the straight parts of the nanobranches. These straight parts look more rough in shape than the branches itself. They are more pronounced built up of elongated aggregates, which are very close together and assemble that way to a fiberlike structure, Fig 4.41(a). This nano-lego phenomenon can also be seen for the high temperature fibers and for MOHP4's nanostructures. The straight parts of the low temperature nanobranches are sometimes made up of two very close rows of elongated aggregates, Fig 4.41(b) branch on the right side. 


\subsubsection{Methoxy-Cyano- $p$-Quaterphenylene (MOCNP4)}

The differently functionalised $p$-quaterphenylene with a methoxy and a cyano group MOCNP4 forms either well defined mutually aligned nanostructures, which remind of walking sticks, or parallely aligned straight nanofibers on a muscovite mica substrate.
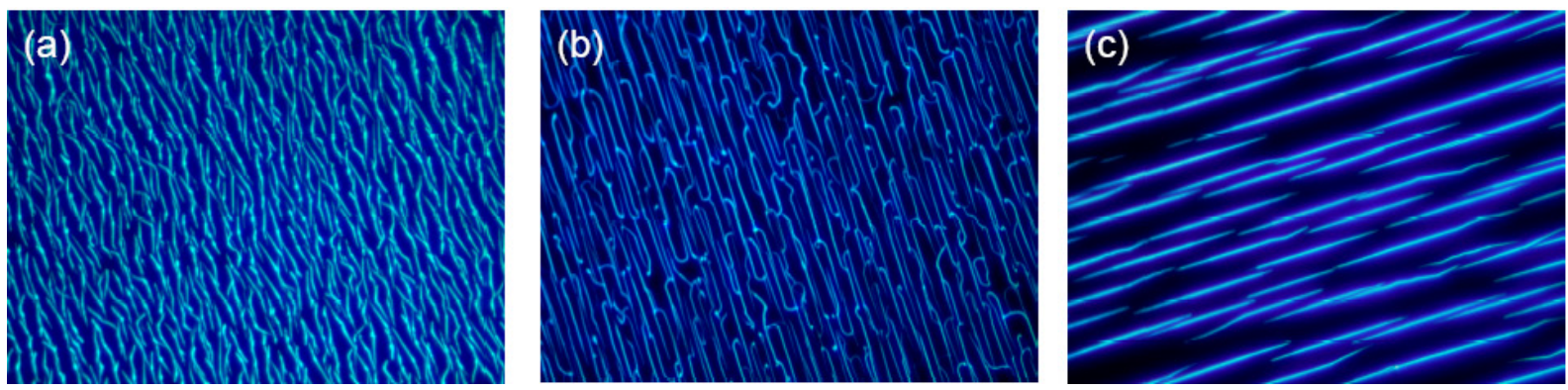

Fig. 4.42: Fluorescence microscopy images of MOCNP4 on muscovite mica (a) $T_{S}=320$ $\mathrm{K}$, film thickness $10 \mathrm{~nm}, 56 \times 42 \mu \mathrm{m}^{2}$, (b) $T_{S}=380 \mathrm{~K}$, film thickness $8 \mathrm{~nm}$, and (c) $T_{S}$ $=390 \mathrm{~K}$, film thickness $10 \mathrm{~nm}$, both $135 \times 100 \mu \mathrm{m}^{2}$.

The main growth direction is along muscovite $\langle 110\rangle$ as usual, and the nanoaggregates show bright, polarised blue fluorescence after excitation with UV light from a high pressure mercury lamp. For comparatively low substrate temperatures $\mathrm{T}_{S}=320 \mathrm{~K}$, Fig 4.42(a), the nanostructures are relatively short, densely packed and less well aligned. Increase of the substrate temperature to $\mathrm{T}_{S}=380 \mathrm{~K}$ during deposition leads to well shaped walking sticks, Fig 4.42 (b). The sticks possess lengths of several ten micrometer. Thus, MOCNP4 nanostructures show a normal temperature dependence in opposition to the symmetrically functionalised oligomer with two cyano groups CNP4.
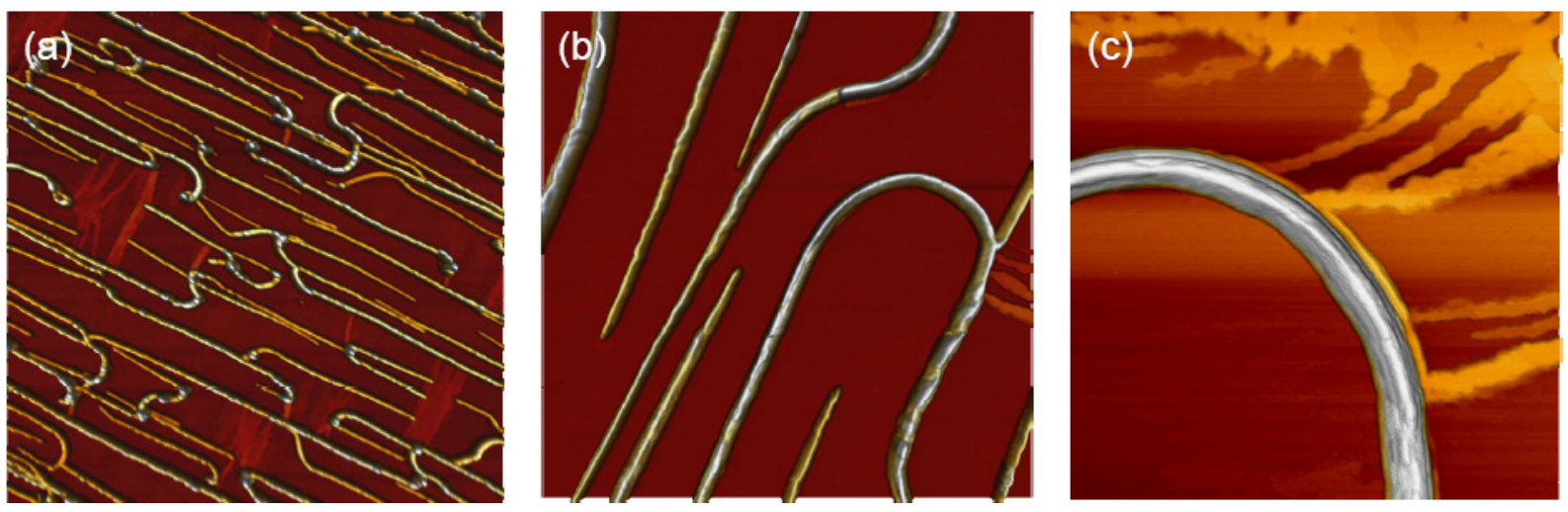

Fig. 4.43: Atomic force microscopy images of MOCNP 4 on muscovite mica (a) $42 \times 42$ $\mu \mathrm{m}^{2}$, height scale $260 \mathrm{~nm}$, (b) $10 \times 10 \mu \mathrm{m}^{2}$, height scale $200 \mathrm{~nm}$, and (c) $5 \times 5 \mu \mathrm{m}^{2}$, height scale $110 \mathrm{~nm}$, all $T_{S}=380 \mathrm{~K}$, film thickness $8 \mathrm{~nm}$.

For a slightly higher substrate temperature, $\mathrm{T}_{S}=390 \mathrm{~K}$, straight, well separeted nanofibers form, Fig 4.42 (c), with length of several ten micrometer or even a few hundred 
micrometer. Only sometimes the nanofibers show kinks. This temperature behaviour is in agreement with basic nucleation theory [167], which predicts larger and more separated crystallites for higher substrate temperatures. But it turned out that more aspects than the substrate temperature are decisive factors, whether walking sticks or straight fibers are formed. The assembly of the MOCNP4 oligomers is clearly more sensitive towards aspects such as substrate quality.
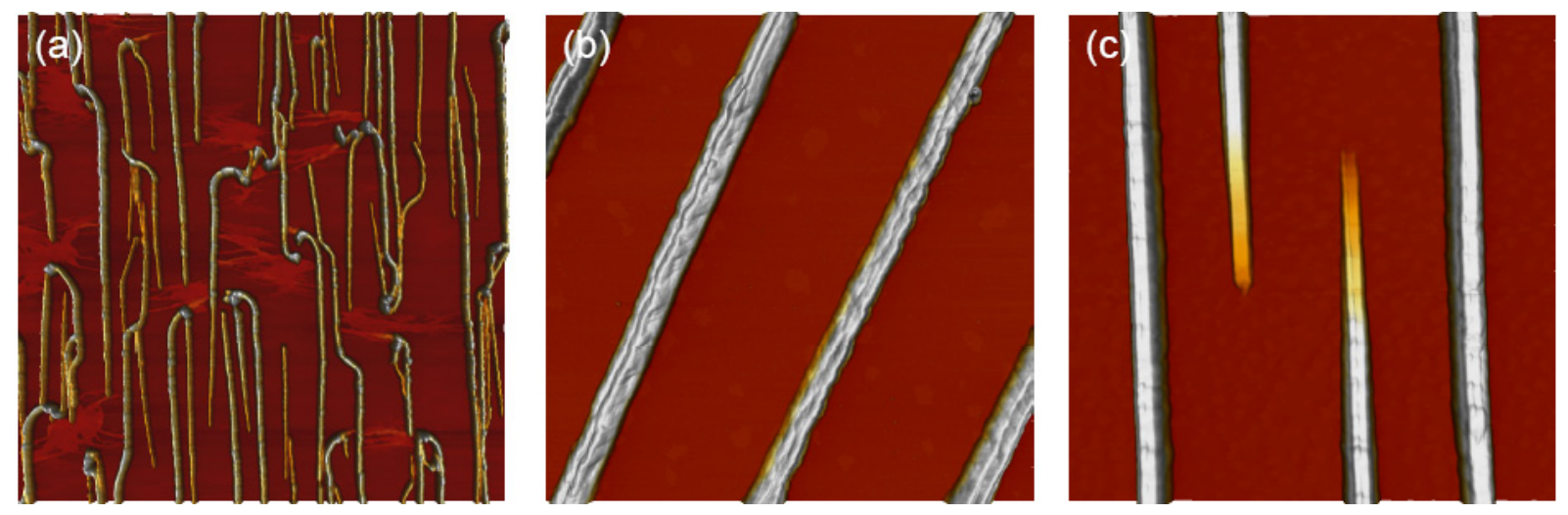

Fig. 4.44: Atomic force microscopy images of MOCNP4 on muscovite mica (a) $42 \times 42$ $\mu \mathrm{m}^{2}$, height scale $250 \mathrm{~nm}$, film thickness $4 \mathrm{~nm}$, (b) $7 \times 7 \mu \mathrm{m}^{2}$, height scale $120 \mathrm{~nm}$, film thickness $8 \mathrm{~nm}$, both $T_{S}=380 \mathrm{~K}$, and (c) $15 \times 15 \mu \mathrm{m}^{2}$, height scale $110 \mathrm{~nm}, T_{S}=390$ $\mathrm{K}$, film thickness $10 \mathrm{~nm}$.

A reasonable explanation can be found by means of atomic force microscopy images. The sticks bend invariably, when large dendritic islands occur on the surface (Fig 4.43). These islands cover several square micrometer and possess heights of several ten nanometer. Step heights of $2.5 \mathrm{~nm}$ indicates, that the islands are formed by upright standing oligomers on the surface. This is in accordance with the fact, that these islands are nonfluorescent in a fluorescence microscope. Since a decrease in substrate quality leads to augmented occurance of upright standing oligomers [172, 173], the number of the islands will increase in turn and all the more bending of the sticks will arise. Islands built up of upright standing oligomers can be found in between the straight fibers as well, but they cover a smaller area and consist only of one or two layers of upright molecules.

That the handhold of the walking sticks consists of a bent fiber piece, has been found out by polarisation measurements of the fluorescence: the polarisation vector follows the bending. This in turn means that the molecules within the bending change their alignment with respect to the substrate and its surface electric fields, similar to the p6P microrings. This is oppositional to the CNP4 swallow wings, whose molecular alignment is strongly given by the substrate unaffected by the nanostructures' shape. However, the fluorescence light emitted from MOCNP4's straight fibers and walking sticks is polarised nearly exactly perpendicular to their growth direction with a polarisation ratio close to 
one. The handhold ends up in a knob, which is about double as high as the stick growing up to $300 \mathrm{~nm}$.

The height of the stick is around $150 \mathrm{~nm}$, and the width is in the order of several hundred nanometer. The straight nanofibers are significantly wider and flatter (Fig.4.44) compared to the sticks, exhibiting heights around $120 \mathrm{~nm}$ an width up to one micrometer. This arises from increased mobility of oligomers and clusters on the surface due to elevated substrate temperature. Both the walking sticks and the straight nanofibers taper off at the endings and elongate in that way. However, this new structure motif of bent fibers with a long straight part opens up more possibilities of implementing this organic nanoaggregates in future integrated optical circuits. 


\subsection{Nanoshaping}

It will be demonstrated in the following chapter, that the fluorescence colour of the nanostructures is determined by the functional group attached to the molecular building block. The fluorescence colour is tunable within the blue. Additionally non-fluorescent nanofibers are available. But this is not all: The detailed morphology, i.e. dimensions, cross-sectional shape, aggregate density, etc. are determinded by the functional groups as well. Atomic force microscopy has been used to visualise the diversity in morphology. The cross-section shape varies from rounded (p6P) over slab-like (MOP4) to peaked like a pitched roof (CLP4), Fig 4.45.
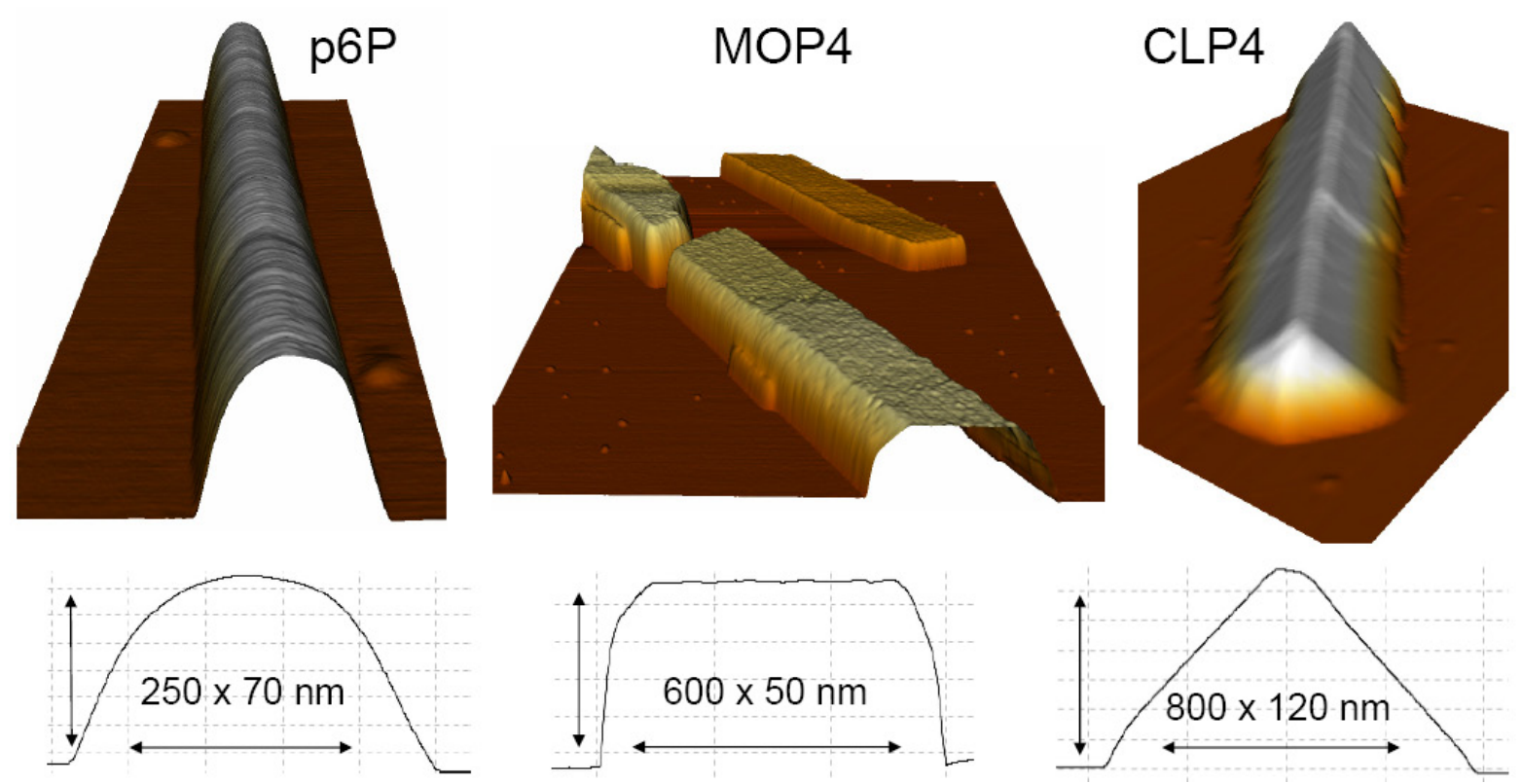

Fig. 4.45: Atomic force microscopy images of nanofibers from p6P, MOP4 and CLP4. Cross-sections (width $\times$ height) show the respective dimensions.

The change in shape occurs for nanofibers, that are still parallely aligned, emit intense blue fluorescence light, and show waveguiding. Hence the nanoshaping opens up various new application possibilities such as slab-based nanophotonic architectures or surface enhanced Raman scattering (SERS) nanosensors, after coating with ultrathin metal films.

The new nanofibers from MOP4 and CLP4 are the most striking candidates for the nanoshaping aspect. Of course other functionalities generate similar shape motifs (Fig 4.46. Introduction of amino groups to the $p$-quaterphenylene core (NHP4) results in rather short, non-fluorescent nanoworms, which possess a rounded cross-sectional shape, while $N, N$-dimethyl amino groups (NMeP4) can lead to exceedingly flat nanoflakes, that emit bright blue fluorescence light. Functionalisation with cyano groups (CNP4) creates rather short and straight nanofibers with a prismatic shaped cross section, among other structure 


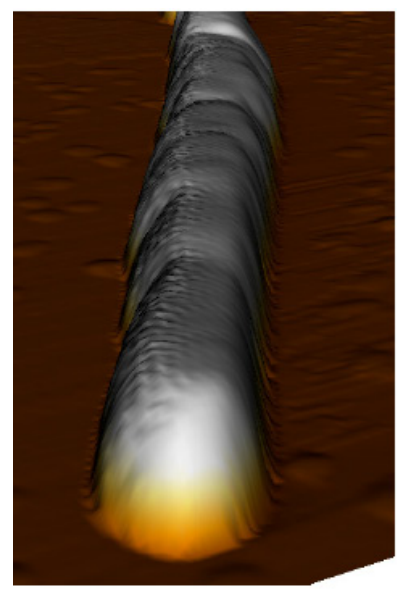

\section{NHP4}
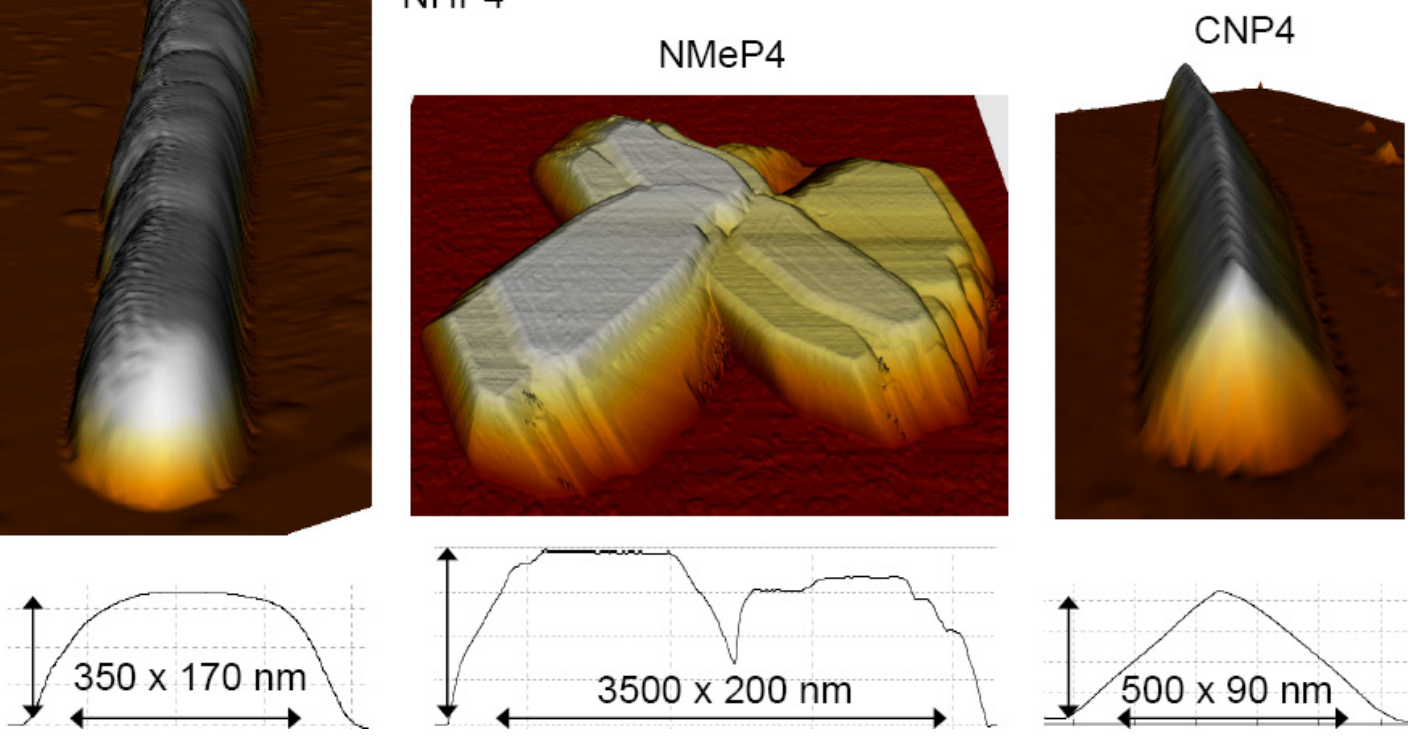

Fig. 4.46: Atomic force microscopy images of nanofibers from NHP4, NMeP4 and CNP4. Height scales $150 \mathrm{~nm}, 250 \mathrm{~nm}, 100 \mathrm{~nm}$, respectively. Cross-sections (width $\times$ height) show the respective dimensions.

types.

Additionally dimesions and aggregate density can be varied according to the growth parameters, e.g. different structure types are generated depending on the substrate temperature during deposition (see above). 
4 Growth - Variety of Nanoaggregates 


\section{Linear Optics}

\subsection{Fluorescence Spectra}

Most nanoaggregates exhibit polarised fluorescence after excitation with unpolarised UVlight $\lambda_{\text {exc }} \approx 365 \mathrm{~nm}$ ) under normal incidence, owing to their high degree of crystallinity. Fluorescence spectra of the para-phenylenes are all within the blue, but the fine-tuning of the emission colour is determined by the functional groups attached to the molecular building block. Functionalisation allows a shift of the peak emission frequency of the nanoaggregates from $383 \mathrm{~nm}$ (CLP4) to $452 \mathrm{~nm}$ (NMeP4). This is due to inductive and mesomeric effects of the functional groups on the conjugated $\pi$-electron system, which is responsible for the fluorescence. While a change in the peak emission frequency via substitution of polymeric or molecular compounds is a well known method in organic thin films, it is demonstrated here, that this is possible while conserving the concept of crystalline nanofibers. The emitted spectra are well resolved and narrow (order of 50 $\mathrm{nm}$ ), and they exhibit no green defect emission due to the crystalline perfection of the nanoaggregates.

The projection of the molecular orientation within the nanostructures on the mica substrate can be extracted by analysing the polarisation properties of the fluorescence after unpolarised UV excitation. This structure determination approach has been discussed in the previous Chapter Growth. Within this section the fluorescence spectra and their shifts due to functional groups is discussed. The spectra have been recorded after continuous wave (cw) UV excitation of the nanoaggregates on the mica substrate at $325 \mathrm{~nm}$. The effect of substituents on the fluorescence properties of aromatic hydrocarbons is complex and additional solid state effects in general are hard to predict. Therefore explanations denoted in the following sections should be viewed cautiously. First some general information on fluorescence and organic molecular crystals are given.

Organic molecules and their molecular crystals, which posess a conjugated $\pi$-electron system exhibit low excitation energies, therefore they absorb in the UV and fluoresce in the visible spectral regime. The $\pi$-electrons are located in the highest energy occupied molecular orbitals (HOMO) and are easily excitable, thus for studying electronic properties of aromatic organic compounds it is sufficient to focus on the properties of the $\pi$-electrons. 


\section{Linear Optics}

Mesomeric effects (M-effects) influence directly the $\pi$-electron system by $\pi$-electrons or lone pairs of the substituent. For inductive effects (I-effects) the electronegativity of the substituent is decisive and those effects cause polarisation of the $\sigma$-bonding between substituent and the corresponding phenyl carbon atom. Mesomeric effects often overcome inductive effects [174]. In general a shift to longer wavelengths of the fluorescence occurs when the degree of conjugation of the $\pi$-electron system is extended or enhanced, i.e. when the conjugated system is elongated or when it becomes more electron-rich. The lowest lying transition of aromatic hydrocarbons are of $\pi \rightarrow \pi^{*}$ type, which are characterised by high absorption coefficients and fluorescence quantum yields. When a heteroatom is involved in the $\pi$-electron system, an $n \rightarrow \pi^{*}$ transition may be the lowest-lying. Promotion of a non-bonding electron of a heteroatom to an antibonding $\pi$-orbital is denoted by $n \rightarrow \pi^{*}$. For such transitions non-radiative de-excitation processes are dominant, which lead to low fluorescence efficiency or even quenching. Fluorescence relevant transitions often possess a significant intramolecular charge transfer character when a heteroatom is involved. Thus, ground states and excited states exhibit different dipole moments. This results in broadened and structureless fluorescence spectra [170].

(a)

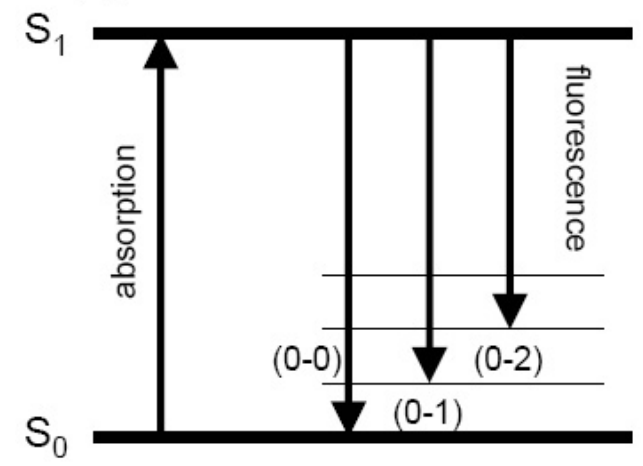

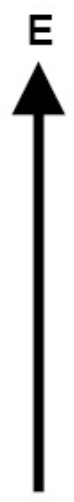

(b)

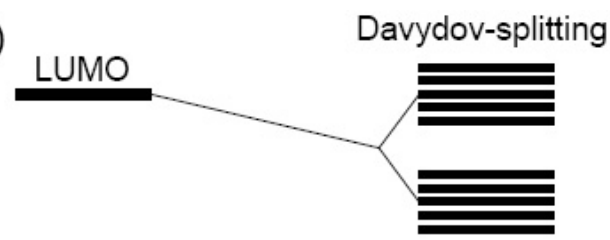

$\mathrm{HOMO}$

single molecule

crystal

Fig. 5.1: (a) Energy terms of molecules and molecular crystals. (b) Molecular crystal with two molecules per unit cell show Davydov-splitting.

The exemplifications given so far are valid for single organic molecules in gasphase or solution. The intermolecular forces, which hold the molecules together in the solid state are weak compared to intramolecular forces. Molecular crystals are named like this, because the molecules with their individual properties are retained in the crystal phase. First analysis of molecular crystal's optical spectra led to the model of the oriented gas [175]. This is the basic difference of organic molecular crystals compared to most anorganic crystals. In the latter ones the individual molecular compounds are not observable. Later the model of Frenkel-excitons [176] and characteristic splitting of spectral lines, the Davydov-splitting [177] have been introduced to explain the influence of intermolecular interactions on the optical spectra of molecular crystals, because they show certain dif- 
ferences compared to the free molecule. For instance the (0-0)-transition (cf. Fig 5.1 (a)) is forbidden for single molecules due to symmetry reasons, whereas it is allowed for the crystal because now the crystal's symmetry is deciding.

Molecular crystals are held together by electrostatic forces, as for instance weak van-derWaals interactions or coulombic forces. Because of repulsion aromatic organic molecules favour the herringbone packing, which allows most densly packing with minimum repulsion [154]. Absoption and fluorescence is characterised by transitions between singletstates $S_{0} \rightarrow S_{1}$ and $S_{1} \rightarrow S_{0}$, respectively, Fig.5.1.(a). The singlet-states consist of different vibronic levels. This model is valid for free molecules and for their crystals. The transitions happen among the $\pi$-electrons.

In general a redshift of absorption and fluorescence occurs when free molecules arrange to a crystal due to intermolecular interactions of all molecules within the crystal. Not only a single molecule is excited but a collective excitation of the bulk of molecules happens. Upon absorption of light an electron-hole pair is formed, the Frenkel-exciton. This is located to a single molecule. Due to intermolecular interactions (coulombic interactions) the exciton can jump to a neighbouring molecule ('hopping'), which results in a delocalised excitation.

Additionally a splitting, the Davydov-splitting, of the excited state can be noticed due to resonance interactions between translationally inequivalent molecules, Fig 5.1(b). The lowest unoccupied molecular orbit (LUMO) splits into two lines for a crystal with two molecules per unit cell such as for p6P. The HOMO does not split due to the weak intermolecular interactions within molecular crystals. For the organic molecule and its crystals in a simple approximation the LUMO refers to the $\pi^{*}$-orbits, and the HOMO to the $\pi$-orbit and sometimes in case existance of functional groups to lone pairs $n$.

For further details on molecular crystals and their excited states see for instance [154, 178 181. However, since properties of single molecules are retained in the molecular crystal, explainations of the nanofibers' optical properties must start with considerations of the oligomers' properties.

\subsubsection{Symmetrically functionalised $p$-Quaterphenylenes}

Fluorescence spectra of the symmetrically functionalised p4Ps' nanoaggregates, all within the blue, can be seen in Fig 5.2. Most of the spectra, apart from CLP4, are redshifted compared to the $\mathrm{p} 4 \mathrm{P}$. The spectra of CLP4 and MOP4 show well resolved vibronic structures, whereas the spectrum of CNP4 is broadened but still shows vibronic structures, and the spectra of the NHP4 and NMeP4 are broad as well as structureless. The vertical line representing the maximum emission, i.e. the (0-1)-transition of the non-functionalised para-quaterphenylene ( $\mathrm{p} 4 \mathrm{P})$, is given for comparison.

The chlorine atom is an electron pulling substituent with a strong-I-effect overcoming a 


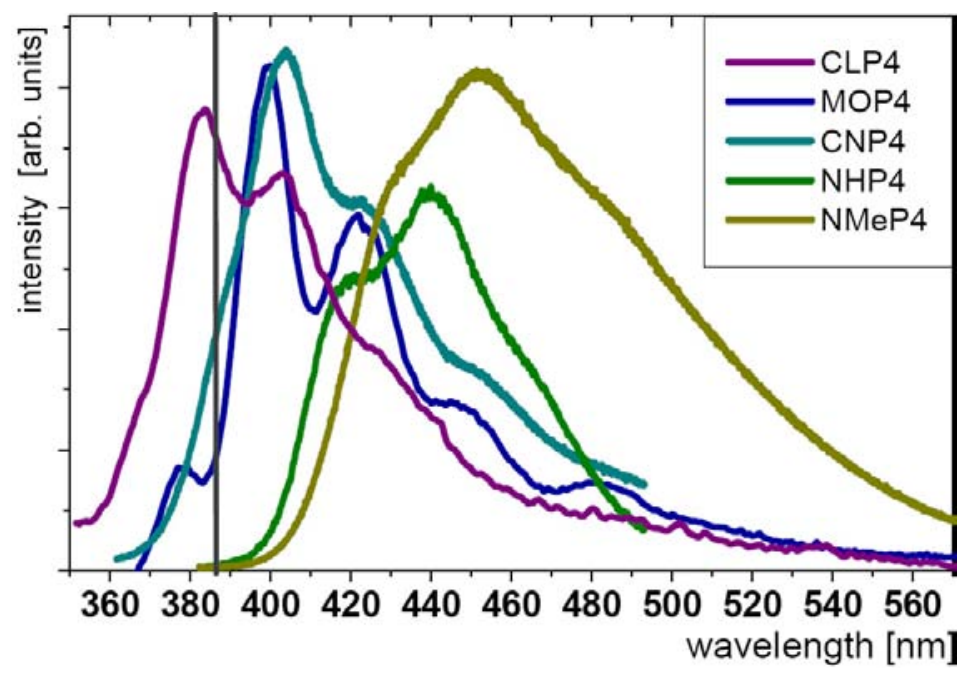

Fig. 5.2: Fluorescence spectra of nanoaggregates from CLP4, MOP4, CNP4, NHP4 and $\mathrm{NMeP} 4$ after $\mathrm{cw}$ excitation at $325 \mathrm{~nm}$. The vertical line represents the $(0-1)$-transition of the p4P. Note that relative intensities are shown, the fluorescence of NHP4's nanofibers is about 100 times weaker compared to the others.

weak + M-effect, therefore electron density of the aromatic system is reduced. This results in a blueshifted fluorescence compared to the parent $\mathrm{p} 4 \mathrm{P}$. The fact that the spectrum of CLP4 shows well resolved vibronic structures indicates that the lone pairs of the chlorine atoms are not directly involved in the aromatic system, i.e. the fluorescence relevant transition has no charge transfer character. The blueshift is clearly due to an inductive effect, since the positive mesomeric effect of the chlorine substituents would result in a contrary shift.

The methoxy group is an electron-pushing functional group, i.e. it has a strong $+\mathrm{M}-$ effect as well as a moderate -I-effect. So the electron density of the conjugated system is increased. This leads to the red-shifted fluorescence of the nanofibers of MOP4 compared to those of the $\mathrm{p} 4 \mathrm{P}$. The spectrum shows well resolved vibronic structures, although spectra of phenols $s^{1}$ are often broad and structureless compared to the parent aromatic hydrocarbon [170]. This loss of structure happens when the lone pairs of the oxygen are directly involved in the $\pi$ bonding of the aromatic system due to significant intramolecular charge transfer character of the fluorescene relevant transitions. This is only possible for the nearly coplanar arrangement of the aromatic ring and the - $\mathrm{OH}$ substituent. However, replacing the hydrogen by alkyl chains, e.g. an -OMe substituent, leads to departure from coplanarity of the aromatic ring and the functional group. Thus, the lone pairs of the oxygen atoms of MOP4 cannot conjugate with the $\pi$-electron system to full extent. The degree of conjugation of the lone pairs is decreased, which reduces the charge transfer character of the fluorescence relevant transitions, so that the vibronic structures are re-

1_-OH functionalised phenyls 
tained on the one hand. But on the other hand the positive mesomeric effect, i.e. the electron pushing effect is still pronounced enough to cause a redshift in the fluorescence.

The cyano group is an electron-pulling substituent with a strong -M- and -I-effect. But it contains a triple bond, which elongates the $\pi$-conjugated system. This overcomes the electron reducing effect, no matter if it is based on negative inductive or on a negative mesomeric effects. Therefore a redshifted fluorescence can be observed compared to the parent $\mathrm{p} 4 \mathrm{P}$. Since a heteroatom in involved in the fluorescence relevant transitions, they possess a certain charge transfer character, because the triple bond of the cyano group is directly involved in the $\pi$-electron system. This results in a broadened spectrum, which still shows vibronic structures. Due to the symmetric functionalisation the charge transfer character is only less pronounced.

The nanostructures of NHP4 have been described as almost non-fluorescent, but still a fluorescene spectrum can be obtained. Note that in Fig 5.2 only relative intensities are given. The fluorescence intensity of NHP4 is about 100 times weaker compared to the other spectra. However, the amino group posseses a strong $+\mathrm{M}$ - and a weak -I-effect: the lone pair of the nitrogen conjugates into the aromatic system, since the N-H-bondings are arranged coplanarly to the phenyl ring. This increases the electron density as well as the conjugation length, which is reflected in an obvious redshift in the fluorescence. This strong positive mesomeric effect overcomes the negative inductive effect by far. Since the lone pairs of the heteroatom are directly involved in the aromatic system, the spectrum is broadened and structureless due to pronounced charge transfer character of the transition momentum. That way no $n \rightarrow \pi^{*}$ transitions are present, which can result in fluorescence quenching as in case of functionalisation with a nitro group (cf. Chapter Growth). Quantum chemical calculations could shed light on this, i.e. orbital energies and most probable electronic transitions could be calculated. However, for NHP4's nanostructures other pathways in fluorescence quenching have to be considered, like for instance interor intramolecular hydrogen bonding of the amino group's hydrogen (cf. Chapter Growth above). That formation of hydrogen bonding is a reasonable explanation is confirmed by the fact, that nanostructures of NMeP4 show bright blue fluorescence.

The spectrum of the nanostructures of the $N, N$-dimethyl amino functionalised oligomer $\mathrm{NMeP} 4$ exhibits the longest wavelength among all investigated functionalised $p$-quaterphenylenes. Similar to the amino group, the lone pair of the nitrogen conjugates into the aromatic system, which results in a redshifted, broadened and structureless fluorescence spectrum. The electron donating effect is more pronounced as in case of the normal amino group due to the positive inductive effect of the methyl groups.

The values of the shifts in the fluorescence spectra are given in Tab 5.1 for a quantitaive overview. 


\begin{tabular}{|c||c|c|c|c|c|c|}
\hline & CLP4 & p4P & MOP4 & CNP4 & NHP4 & NMeP4 \\
\hline \hline$(0-1)[\mathrm{nm}]$ & 383 & 386 & 400 & 404 & 439 & 452 \\
\hline \hline$\Delta \lambda[\mathrm{nm}]$ & -3 & 0 & +14 & +18 & +53 & +66 \\
\hline
\end{tabular}

Tab. 5.1: Fluorescence shifts of symmetrically functionalised $p$-quaterphenylenes with respect to the unfunctionalised $\mathrm{p} 4 \mathrm{P}$. Differences $\Delta \lambda$ are given in $\mathrm{nm}$; minus and plus indicate blue- and redshift, respectively. The wavelength of the (0-1) transitions are given in $\mathrm{nm}$ as well.

\subsubsection{Mono functionalised $p$-Quaterphenylenes}

For the mono functionalised oligomers intermolecular interactions are more pronounced than for $p$-quaterphenylenes with functional groups at both para-positions due to the possiblity of intermolecular interaction between hydrogen and functional group. These kind of hydrogen bondings have been recognised as important interactions in supramolecular chemistry [182]. Unfortunately, no crystal structures have been obtained yet for the functionalised $p$-quaterphenylenes to verify these interactions. However, the fluorescence spectra reflect increased intermolecular interactions, and the nanofibers' capability of frequency doubling (cf. Chapter Non-linear Optics) gives an evidence for head-to-tail orientation of the oligomers within the nanofibers' assumed herringbone packing. General aspects of the respective functional groups on the fluorescence properties have already been discussed in the previous section. The spectra of the mono functionalised oligomers' nanofibers, all within the blue and redshifted compared to the $\mathrm{p} 4 \mathrm{P}$, can be seen in Fig 5.3 . again the spectra of CLHP4 and MOHP4 show well resolved vibronic structures, whereas the other spectra are broad and structureless. The vertical line represents the maximum emission, i.e. the (0-1)-transition of the unfunctionalised para-quaterphenylene (p4P).

\begin{tabular}{|c||c|c|c|c|c|c|c|c|}
\hline$[\mathrm{nm}]$ & CLP4 & CLHP4 & MOHP4 & MOP4 & CNP4 & CNHP4 & NMeHP4 & NMeP4 \\
\hline \hline$(0-1)$ & 383 & 390 & 397 & 400 & 404 & 417 & 440 & 452 \\
\hline \hline X-P4-X & -3 & & & +14 & +18 & & & +66 \\
\hline X-P4-H & & +4 & +11 & & & +31 & +54 & \\
\hline
\end{tabular}

Tab. 5.2: Fluorescence shifts of symmetrically and mono functionalised $p$ quaterphenylenes with respect to the unfunctionalised p4P. Differences $\Delta \lambda$ are given in $\mathrm{nm}$; minus and plus indicate blue- and redshift, respectively, with respect to $\mathrm{p} 4 \mathrm{P}$. The wavelength of the (0-1) transitions are given in $\mathrm{nm}$ as well. 

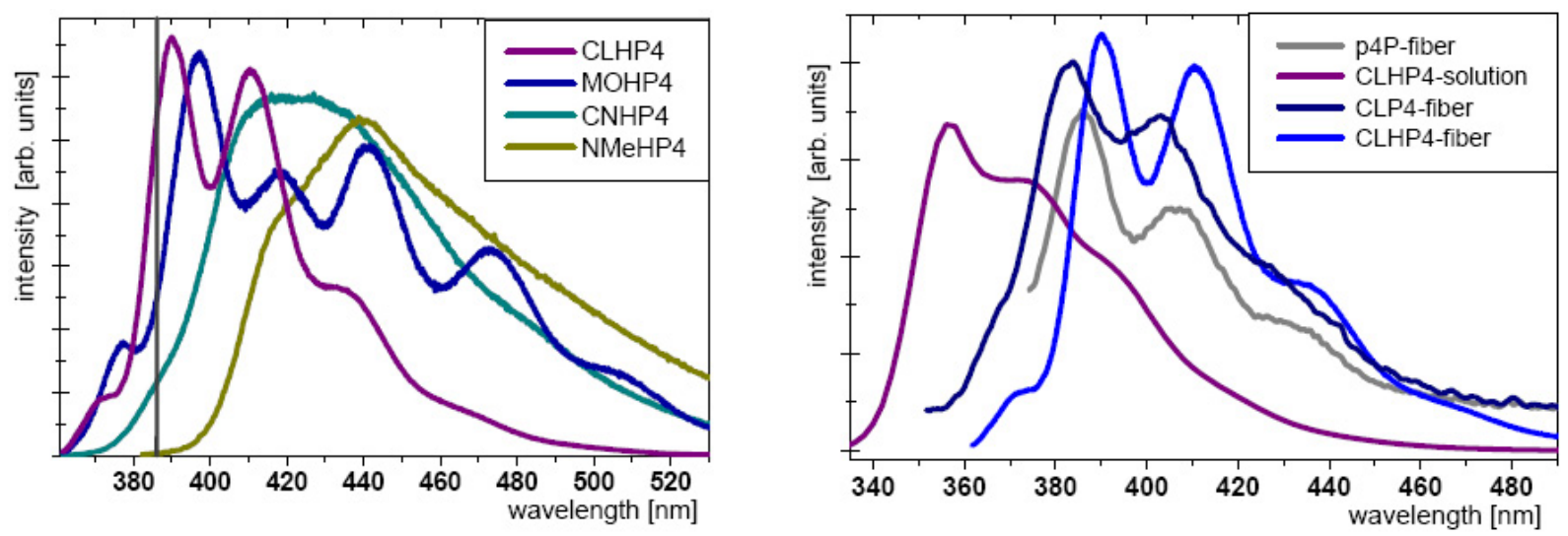

Fig. 5.3: Fluorescence spectra of nanoaggregates from CLHP4, CNHP4, MOHP4 and NMeHP4 after $\mathrm{cw}$ excitation at $325 \mathrm{~nm}$. The vertical line represents the (0-1)-transition of the p4P (left hand side). Exemplarily fluorescence spectra of p4P-fibers, CLP4-fibers, CLHP4-fibers and CLHP4 in solution are shown together in one plot (right hand side).

The mono functionalised oligomer MOHP4 leads to nanofibers with a red-shifted fluorescence compared to the parent $\mathrm{p} 4 \mathrm{P}$. This redshift is also found in case of the disubstituted oligomer MOP4, but marginally more pronounced. This is in accordance with the simple fact, that the electron donating effects of the methoxy groups will add up in case of di-functionalisation.

The chlorine atom as an electron-pulling substituent reduces electron density of the aromatic system on the one hand, but on the other hand the lone pairs of the chlor atom can undergo intermolecular interactions: The lone pair can interact with a hydrogen of the adjacent molecule forming hydrogen bondings, if the molecules align head-to-tail within the nanoaggregates' assumed herringbone packing2 ${ }^{2}$. Hydrogen bondings to heteroatoms are known to affect fluorescence properties [170]. The $\pi-\pi^{*}$ state can shift to lower energy, whichs results in a red-shift of the fluorescence. Additionally the $n-\pi^{*}$ state can shift to higher energy, which can increase fluorescence quantum yield because emission from $n-\pi^{*}$ states is known to be less efficient than from $\pi-\pi^{*}$ states. This is in agreement with the extraordinary bright fluorescene of the CLHP4's nanofibers (see below). In case of the difunctionalised oligomer with chloride groups (CLP4) the fluorescence of the nanofibers is blueshifted compared to the fluorescence of the $\mathrm{p} 4 \mathrm{P}$. This is due to the reduction of electron density because of the negative inductive effect of the chloride groups. No intermolecular interactions as believed for the case of the CLHP4 can occur. The fact that the spectra of both chloride functionalised oligomers show well resolved vibronic structures indicates that the lone pairs of the chlor atoms are not directly involved in the aromatic system,

\footnotetext{
${ }^{2}$ This orientation leads to a certain non-symmetry of the nanofibers, which is crucial for non-linear optical activity such as frequency doubling. Appearance of these features are disclosed in the Chapter Non-linear Optics, which give an indirect evidence for the here quoted intermolecular interactions.
} 


\section{Linear Optics}

i.e. the fluorescence relevant transition has no charge transfer character.

The CNHP4's nanofibers fluorescence shows a pronounced redshift with respect to the parent $\mathrm{p} 4 \mathrm{P}$ as well as to the respective symmetrically functionalised oligomer, although the di-functionalised oligomer CNP4 should have a more elongated conjugated system because of the second cyano group. This could be explained by the electron-pulling effect due to a negative inductive as well as a negative mesomeric effect of the cyano group. The second cyano groups then decreases the electron density of the phenylene core more than it increases the length of the conjugated system. In addition the cyano group also contains a lone pair, which can interact with hydrogen of a neighbouring oligomer in case of head-totail orientation of the CNHP4 oligomers. The formation of such intermolecular hydrogen bondings should result in a red-shifted fluorescence as discussed above. The spectrum of CNHP4 is broad and structureless due to the charge tranfer character of the fluorescence relevant transitions, because the triple bond of the cyano group is directly involved in the aromatic system.

The strong positive mesomeric effect of the $N, N$-dimethyl amino group, which easily overcomes the negative inductive effect is reflected in an obviously redshifted fluorescence of the NMeHP4's nanofibers. This redshift is clearly more pronounced for the di-substituted oligomer $\mathrm{NMeP} 4$ due to the second $N, N$-dimethyl amino group. Intermolecular interactions as believed for CLHP4 and CNHP4 can be neglected because of the low basicity of aromatic amines and because of steric reason due to the methyl groups. The spectrum of the NMeHP4's nanostructures is broadened and structureless due to the known mesomeric effects of lone pairs of amino groups.

The table Tab. 5.2 gives a quantitative overview over the fluorescence shifts of both, the mono and the symmetrically functionalised oligomers's spectra.

\begin{tabular}{|c||c|c|c|c|c|c|c|c|}
\hline$[\mathrm{nm}]$ & $\mathrm{CLH}_{s}$ & $\mathrm{CLH}_{f}$ & $\mathrm{MOH}_{s}$ & $\mathrm{MOH}_{f}$ & $\mathrm{CNH}_{s}$ & $\mathrm{CNH}_{f}$ & $\mathrm{NMeH}_{s}$ & $\mathrm{NMeH}_{f}$ \\
\hline \hline$(0-1)$ & 357 & 390 & 385 & 397 & 390 & 417 & 447 & 440 \\
\hline \hline solution & -29 & & -1 & & +4 & & +61 & \\
\hline fiber & & +4 & & +11 & & +31 & & +54 \\
\hline
\end{tabular}

Tab. 5.3: Fluorescence shifts of mono functionalised $p$-quaterphenylenes in solution (dichloromethane) and of the respective nanofibers with respect to the unfunctionalised p4P's nanoaggregates. Subscript $s$ indicates solution and subscript $f$ indicates fiber. Differences $\Delta \lambda$ are given in nm; minus and plus indicate blue- and redshift, respectively, with respect to $\mathrm{p} 4 \mathrm{P}$. The wavelength of the (0-1) transitions are given in $\mathrm{nm}$ as well.

Additionally fluorescence spectra of the mono functionalised p4Ps have been recorded in solution by Ivonne Wallmann, University of Bonn [2], see Tab.5.3. The oligomers were dissolved in dichloromethane in high dilution (concentration $<1 \mu \mathrm{M}$ ), therefore in- 
termolecular interactions of the molecules such as considered for the nanofibers can be neglected. Exemplary all available fluorescence spectra of the mono and symmetrically chloride functionalised p4P are shown together in one plot, Fig 5.3 . The differences between the maximum emission of all oligomers in solution and of the respective nanofibers are found as following:

\begin{tabular}{c|cccc} 
oligomer & CLHP4 & MOHP4 & CNHP4 & NMeHP4 \\
\hline$\Delta[\mathrm{nm}]$ & 33 & 12 & 27 & 7
\end{tabular}

Obviously the nanofibers of CLHP4 and CNHP4 show a more pronounced redshifted fluorescence in contrast to the oligomers in solution. This is in accordance with the believed intermolecular hydrogen bondings of CLHP4 and CNHP4 within the nanofibers, which are responsible for a fluorescence shift to higher wavelength. The difference between the maximum emission of the oligomers in solution and the respective nanofibers in cases of MOHP4 and NMeHP4 is about three times lower than for the other two oligomers.

For NMeHP4 in solution the maximum fluorescence is even located at a higher wavelength than the maximum of the NMeHP4's nanofibers. This can be explained by a solvent effect since dichloromethane is a rather polar solvent. Because of the pronounced charge transfer character of the fluorescence relevant transitions the increase in the dipole moment for the excited state is quite large. Consequently the excited state is not in equilibrium with the surrounding solvent molecules. The solvent molecules rotate during the lifetime of the excited state until the solvation shell is in thermodynamic equilibrium with the fluorophore. Such a solvent relaxation explains the increased redshift of the fluorescence for increasing polarity of the solvent [170].

The three other oligomers in solution have a blueshifted fluorescence compared to the respective nanostructures. This reflects not only the missing intermolecular interactions but also the departure from coplanaity of the adjacent phenyl rings. The phenyl rings are twisted with respect to each other for para-phenylenes in solutions due to repulsion of the hydrogens bond to the aromatic ring. The assumed herringbone structure of the molecules within the nanoaggregates forces the phenyl rings into a nearly coplanar arrangement, which provides a better conjugation across the molecules and hence results in a redshifted fluorescence.

Also a qualitative order of the fluorescence intensities is given:

$$
\text { ClHP4 > CNHP4 > MOHP4 > NMeHP4 }
$$

The intensities are strongly influenced by the morphology of the aggregates and the nominal film thicknesses. So only a cautious qualitative comparison is made. The nanoaggregates of CLHP4 show much more intense fluorescence about ten times more intense under similar conditions than the fluorescence of the nanofibers from CNHP4. But this 


\section{Linear Optics}

luminescence is itself still about ten times stronger than the fluorescence of the nanostructures from MOHP4. The luminescence of the nanofibers from NMeHP4 is about half as intense compared to those of MOHP4.

\subsubsection{Differently functionalised $p$-Quaterphenylenes}

Fluorescence spectra of the differently functionalised $p$-quaterphenylenes' nanofibers are shown in Fig.5.4. The spectrum of the parent p4P oligomer is given for comparison. All spectra are clearly redshifted with respect to the parent $\mathrm{p} 4 \mathrm{P}$ 's fluorescence. Effects of two different functional groups on both para-positions are complex. It seems that the effect of introducing auxochromic groups is more pronounced than the individual properties of the substituent: The respective peak emissions of all three different nanofibers are close together and clearly red-shifted.

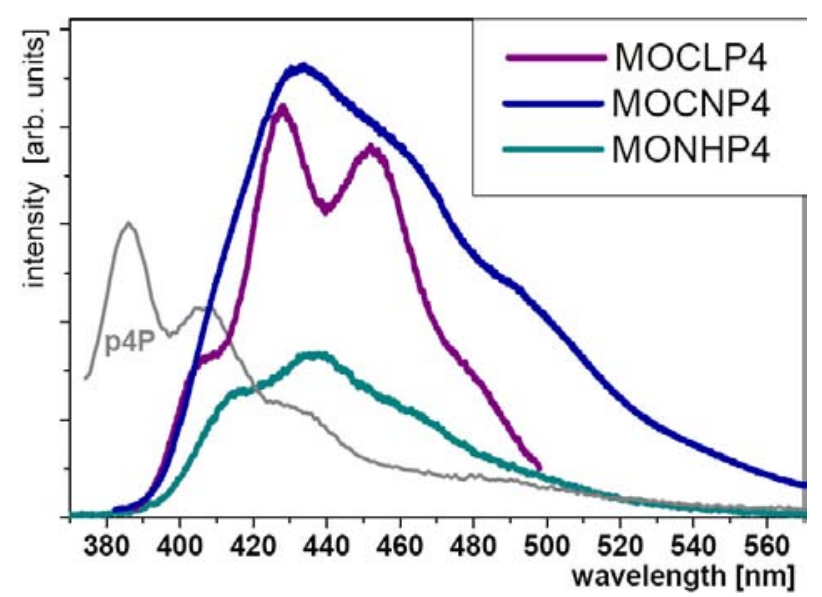

Fig. 5.4: Fluorescence spectra of nanoaggregates from MOCLP4, MOCNP4 and MONHP4 after cw UV excitation at $325 \mathrm{~nm}$. A spectrum of $\mathrm{p} 4 \mathrm{P}$ is given for comparison. Note that relative intensities are shown, the fluorescence of MONHP4's nanofibers are about 100 times weaker compared to the others.

\begin{tabular}{|c||c|c|c|c|}
\hline & p4P & MOCLP4 & MOCNP4 & MONHP4 \\
\hline \hline$(0-1)[\mathrm{nm}]$ & 386 & 428 & 433 & 438 \\
\hline \hline$\Delta \lambda[\mathrm{nm}]$ & 0 & +42 & +47 & +52 \\
\hline
\end{tabular}

Tab. 5.4: Fluorescence shifts of differently functionalised $p$-quaterphenylenes with respect to the unfunctionalised p4P. Differences $\Delta \lambda$ are given in $\mathrm{nm}$; minus and plus indicate blue- and redshift, respectively. The wavelength of the (0-1) transitions are given in $\mathrm{nm}$ as well.

Since one of the functional groups is the same for all three oligomers, namely the 
methoxy group, the respective influence of the second substituent can be discussed. The MOCLP4 nanofibers show the most blue fluorescence due to the electron pulling chlorine atom. Substitution with a cyano group causes elongation of the $\pi$-electron system on the one hand, but reduces electron density due to electron reducing effects. Therefore the MOCNP4 nanofibers have the middle position here, whereas the MONHP4 nanofibers exhibit the most redshifted fluorescence because of the pronounced positive mesomeric effect of the amino group. The amino group arranges coplanarly to the phenyl rings so that the lone pair of the amino group is directly involved in the aromatic system. An overview over the shifts in the fluorescence spectra of the differently functionalised oligomers' nanofibers is given in Tab 5.4 .

Only the spectrum of MOCLP4 nanofibers shows well resolved vibronic structures. This is in good agreement with the results for the symmetrically and mono functionalised compounds. The fluorescence relevant transitions exhibit a significantly charge transfer character, if a heteroatom is involved directly in the conjugated system, which is the case for the cyano and the amino substituent.

Since the MONHP4 nanofibers have been described as non-fluorescent in a fluorescence microscope, it has to be pointed out that the here presented fluorescence is about 100 times weaker compared to the fluorescence of the other nanoaggregates. This fluorescence quenching is attributed to the formation of intermolecular hydrogen bonding between the hydrogen of the amino groups and the lone pairs of the methoxy group's oxygen. The molecules are believed to orientate in head-to-tail configuration, which is also reflected in the non-linear optical activity (see below). However, this problem of fluorescence quenching has been found in case of NHP4's nanofibers and is well known for naphthalene bisimide dyes core-substituted with arylamines: Formation of intramolecular hydrogen bonding to a neighbouring carbonyl group is considered to be the pathway for radiationless deactivation of the excited state. To avoid this problem alkyl amines [168] and aminolinked benzyl groups [169], respectively, have been attached sucessfully to the naphthalene core leading to a strong, tunable fluorescence. 


\section{$5.2 \mathrm{UV} /$ vis Absorption Spectra}

$\mathrm{UV} /$ vis absorption spectra of the symmetrically and differently functionalised $p$-quaterphenylenes in solution (dichloromethane) have been measured as well, Fig.5.5. The values for the maximum absorption are given in Tab.5.5. The solutions are highly diluted (concentrations around $50 \mathrm{nM}$ ), therefore no intermolecular interactions influence the absoption - the absorption spectrum can be considered as overlap of spectra of single molecules. The spectra are structureless and rather broad as is known for substituted benzenes [183], because substituents reduce symmetry and enlarge the chromophoric system. The fluorescence of the nanofibers, i.e. interacting crystalline bulk of oligomers, can easily be excited with UV light at $365 \mathrm{~nm}$, wheras for excitation of the isolated oligomers in solution a lower wavelength is needed. This reflects that the oligomers' phenyl rings are not coplanar but twisted in solution. Departure from coplanarity results in a blueshift of the $\pi$-system's absorption maximum.
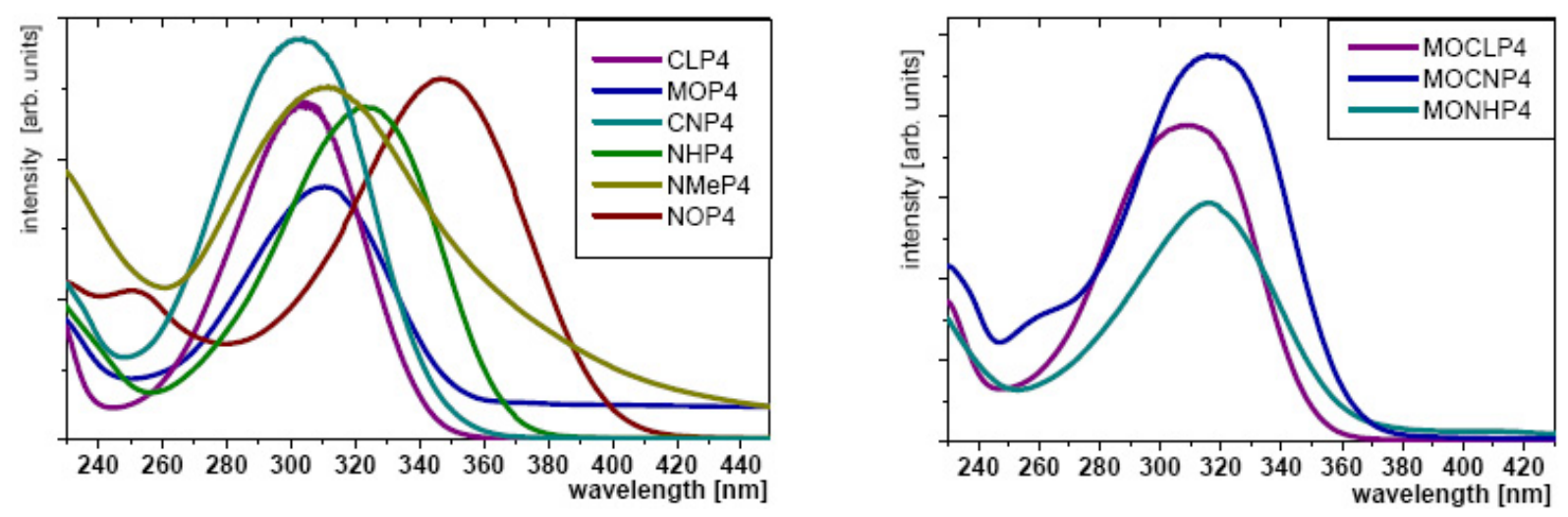

Fig. 5.5: UVvis absorption spectra of CLP4, MOP4, CNP4, NHP4, NMeP4 and NOP4 (left) and of MOCLP4, MOCNP4 and MONHP4 (right) in dichloromethane solution. Concentrations are around $50 \mathrm{nM}$.

The effect of the functional group on the absorption properties of oligomers in solution is less pronounced than the effect on the fluorescence properties for the nanofibers. This may also be due to the twisted arrangement of the phenyl rings for oligomers in solution: The impact of the functional group on the aromatic system cannot 'spread' as easily as for the phenyl rings' coplanar arrangement for the bulk within the nanofibers. However, introduction of functional group leads to a redshift in the absoption spectra due to elongation of the chromophoric system. Electron pulling functional groups like chloride and cyano groups cause a relatively small redshift, while electron donating functional groups like methoxy, amino and $N, N$-dimethyl amino groups cause a larger redshift. For the differently functionalised oligomers the influence of the two different substituents is complex, therefore the simple considerations for the symmetrically substituted oligomers are not valuable. Apart from this, the nitro group has an exceptional position: the maximum 
absorption of NOP4 is clearly redshifted compared to all other oligomers. This is due to strong chromophoric properties of the nitro group.

\begin{tabular}{|c||c|c|c|c|c|c|c|c|c|c|}
\hline & p4P [93] & CL & MO & CN & NH & NMe & MOCL & MOCN & MONH & NO \\
\hline \hline$(0-1)$ & 299 & 303 & 310 & 302 & 324 & 311 & 309 & 318 & 316 & 347 \\
\hline \hline$\Delta \lambda$ & 0 & +4 & +11 & +3 & +25 & +12 & +10 & +19 & +17 & +48 \\
\hline
\end{tabular}

Tab. 5.5: Absorbance shifts of symmetrically and differently functionalised $p$ quaterphenylenes with respect to the unfunctionalised $\mathrm{p} 4 \mathrm{P}$ in dichloromethane solution (around $50 \mathrm{nM}$ ). 'P4' has to be inserted at the end of the oligomer's name. Differences $\Delta \lambda$ are given in nm; plus indicates redshift. The wavelength of maximum absorption is given as well. 


\subsection{Bleaching [7, 8]}

Although it has not been investigated by the author, it has to be noted that the above discussed fluorescence of the nanofibers undergoes photodegredation, the so called bleaching. For the functionalised oligomers nanofibers the bleaching has been studied in detail in case of MOP4, a short overview is given in the following.

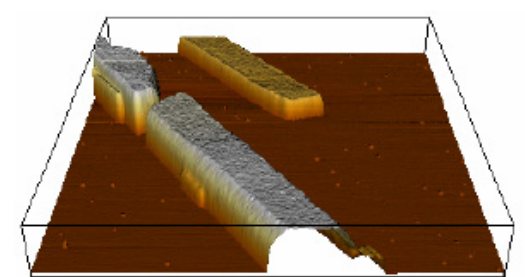

$0 \mathrm{~min}$

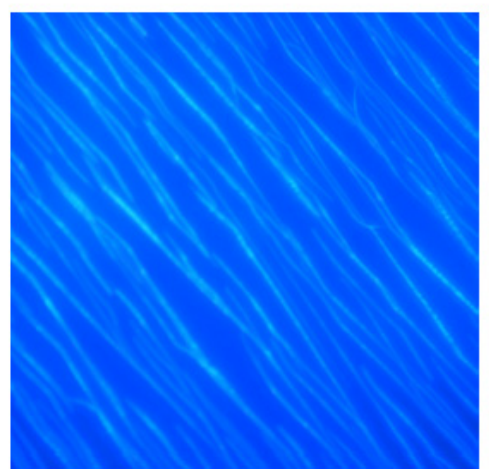

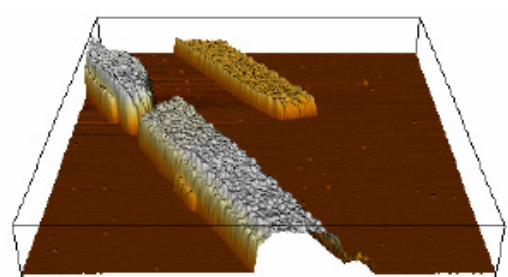

$3 \mathrm{~min}$

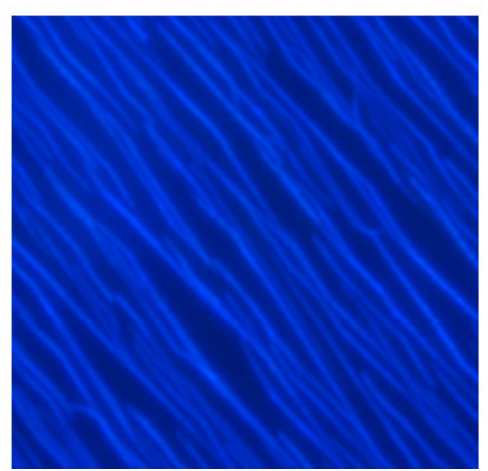

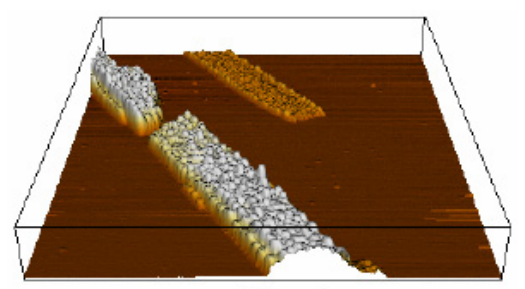

$13 \mathrm{~min}$

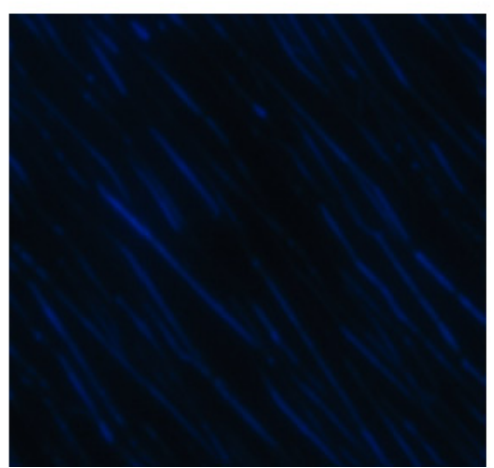

Fig. 5.6: Bleaching: atomic force microscopy images $\left(3.7 \times 3.7 \mu \mathrm{m}^{2}\right.$, height scale $\left.65 \mathrm{~nm}\right)$ and corresponding fluorescence microscopy images $\left(250 \times 250 \mu \mathrm{m}^{2}\right)$ of MOP4 nanofibers on muscovite mica before and after illumination with $365 \mathrm{~nm}$ UV light for the indicated times Adopted from [7].

Before applications of this newly generated nanofibers in photonic and optoelectronic devices will be possible, the stability against heat treatement and optical bleaching has to be investigated and improved. It has been demonstrated in the case of p6P nanofibers, that the fluorescence spectra do not change for thermal heating up to around $400 \mathrm{~K}$ under ambient air conditions [7]. For higher temperatures the fibers degrade by loosing material. But still the shape of the fluorescence spectra does not change, only the luminescence intensity decreases until all organic material is removed. The bleaching caused by UV light illumination has been investigated in more detail in case of $\mathrm{p} 6 \mathrm{P}$ and $\mathrm{MOP} 4$ nanofibers. For better understanding the change on morphology induced by bleaching with UV light, AFM measurements have been performed simultaneously on the illuminated area for MOP4 [7]. Fig 5.6 shows fluorescence microscopy images and the corresponding AFM images of MOP4 nanofibers on mica before and after illumination with $365 \mathrm{~nm}$ UV light. With increasing illumination time material is ablated from the fibers, which results in a decrease in height and an increase in surface roughness. A quantitative evaluation of 
the bleaching process reveals that the decrease in height goes linearly with time and is accompanied by an exponential decrease in luminescence intensity. Since it is only the height of the nanofibers that decreases while the width remains approximately constant, the volume of the fibers decreases linearly with illumination time as well. One would expect that the luminescence intensity should decrease linearly with illumination time if the luminescence results from an integration of all emitters within the fiber. But the luminescence decays exponentially with illumination time, thus the bleaching process is more than ablation of organic material. Since illumination and optical measurements took place from below, i.e. through the mica substrate, photo-oxidation reaction must affect the whole fiber and not only its surface.

But bleaching can be avoided or at least largely reduced if the nanofibers have been coated with for instance silicon oxide before illumination. The $\mathrm{SiO}_{x}$ coating is very effective in blocking ambient oxygen, which undergoes photo-oxidation reactions with the organic material upon illumination with UV light.

Another important step towards device application for nanofibers is to remove them from the growth substrate, while conserving the nanofibers' shape and optical properties. A convenient transfer process for these kind of nanofibers has already been developed [184, 185]. The presence of the substrate might have an influence on the optical properties of the fibers. However, it has been found out, that the transfer process from the growth substrate to a liquid does not change the morphology or the general optical properties of the nanofibers such as their ability to emit anisotropic blue light and their waveguiding properties [185]. 
5 Linear Optics 


\section{Non-linear Optics [6, 9]}

Non-symmetrically substitution of oligomers should not only allow tuning of the luminescence behaviour but also give access to systems that show nonlinear optical (NLO) properties. The differently functionalised oligomers MONHP4, MOCLP4 and MOCNP4 are (to a different extent) push-pull substituted systems as well as the mono functionalised oligomers MOHP4, CLHP4, NMeHP4 and CNHP4. They can act as NLO prototypes, since they are expected from theory to posses non-zero second order susceptibility due to the non-symmetric functionalisation [83 85]. If this anisotropy is retained in the highly crystalline nanoaggregates, which should be the case for head-to-tail orientation of the molecules within the nanofibers' assumed herringbone packing, this bulk of oligomers should also exhibit NLO activity. Such a property is of obvious importance for future integrated optical circuits, but optical second-harmonic generation (SHG) is also a powerful technique for understanding the correlation between morphology and optoelectronic response of nanoaggregates and for characterisation.

In general the induced polarisation $\vec{P}$ by an external field describes the optical response of a medium. In the linear optical regime, i.e. for relatively weak applied electromagnetic fields, the relation between $\vec{P}$ and the external electric field $\vec{E}$ is linear:

$$
\vec{P}=\chi \cdot \vec{E}
$$

The susceptibility tensor $\chi$ is a material parameter.

In the nonlinear optical regime, the response function $\chi$ now becomes dependent on the electromagnetic field $E$, and the induced polarisation can be written as an expansion of the electric field [186]:

$$
\begin{aligned}
\vec{P} & =\chi^{(1)} \cdot \vec{E}+\chi^{(2)} \cdot \vec{E} \vec{E}+\chi^{(3)} \cdot \vec{E} \vec{E} \vec{E}+\ldots \\
& =\vec{P}^{(1)}+\vec{P}^{(2)}+\vec{P}^{(3)}+\ldots
\end{aligned}
$$

$\chi^{(n)}$ is the $\mathrm{n}$-th order susceptibility tensor. Hence, in the nonlinear optical experiment, the light 'retransmitted' from the material contains not only the fundamental (input) frequency, but also the double frequency (SHG), the triple frequency (third-harmonic generation), and so on.

Due to symmetry reasons $\chi^{(n)}$ vanishes for even- $n$ in media with inversion symmetry: In a parity operation the electric fields $\vec{E}$ change algebraic sign, $\vec{P}(-\vec{E})=-\vec{P}(\vec{E})$. This 
constraint can only be fulfilled if the even terms vanish in Eqn 6.3. Only when the inversion symmetry is broken, such as at surfaces or interfaces or in anisotropic media, an even-order $\chi^{(n)}$ is no longer forbidden. This allows nonlinear optical processes like frequency doubling.
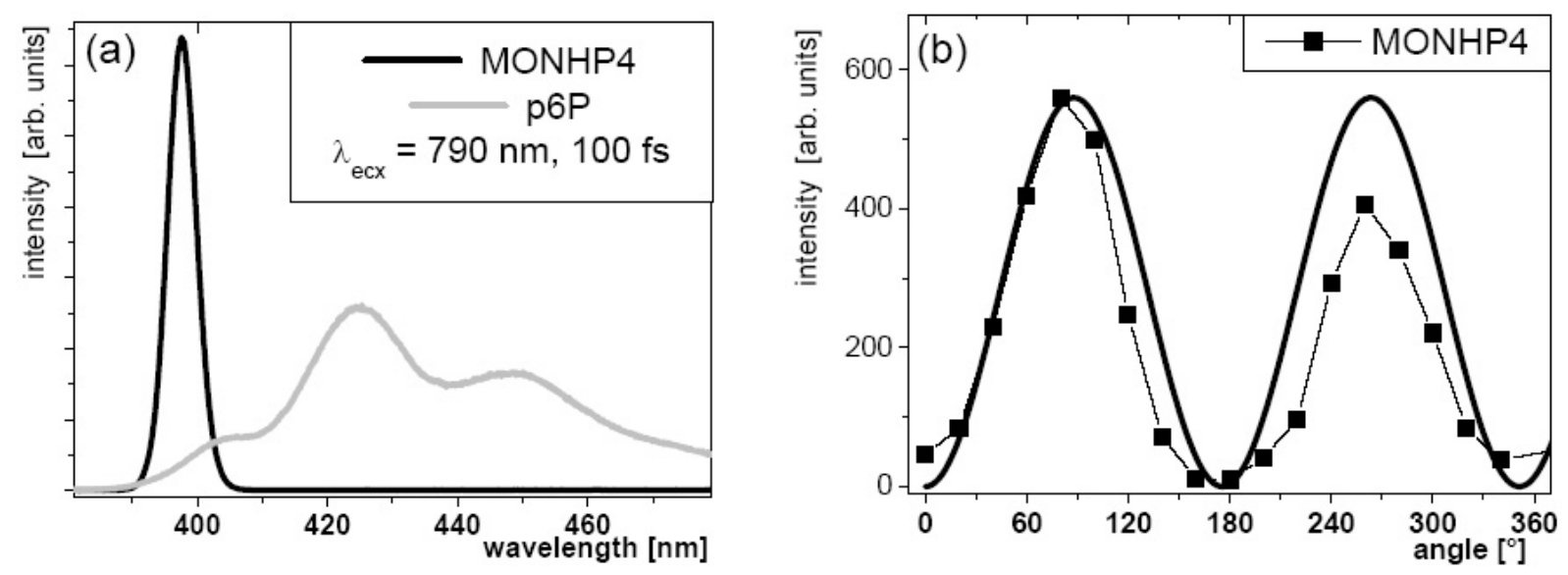

Fig. 6.1: (a) Emission of second harmonic signal from transferred MONHP4 nanofibers and from p6P nanofibers on muscovite mica after excitation with femtosecond near infrared laser pulses (100 fs, $4.5 \mathrm{~mW}$ ) at $790 \mathrm{~nm}$. (b) Polarisation dependence of the SH-signal from transferred MONHP4 nanofibers as a function of the rotational angle. Solid line is a $\sin ^{2}$ fit.

It has been reported before that nanofibers from para-hexaphenylene exhibit nonlinear optical activity, but this can mainly be assigned to surface second harmonic generation from a continuous organic film (wetting layer) on the growth substrate and from the nanofiber surfaces [187], according to recent investigations on transferred nanofibers [9]. The amount of true second harmonic generation from the nanofibers of $p$-hexaphenylene is reported to be in order of a few percent of the two-photon luminescence intensity at infrared femtosecond excitation around $800 \mathrm{~nm}$.

The non-symmetrically functionalised $p$-quaterphenylenes, however, behave differently. For the observation of the fibers' intrinsic properties, nanofibers obtained from MONHP4 have been transferred from mica to glass (silicon oxide) using a standard procedure [184, 185] in order to avoid second-harmonic generation from the underlying substrate and a wetting layer.

The nonlinear optical properties of the nanofibers have been investigated by Jonathan Brewer [188], University of Southern Denmark, with two different approaches (see Chapter Experimental for details): (I) For measurements of polarised optical spectra, a femtosecond pulsed laser was used to excite the nanofibers at different wavelength within the near infrared. (II) A laser scanning confocal microscope (LSM) coupled with a Ti:sapphire laser 
for two photon excitation experiments served to obtain three-demensional SHG images of individual needdles. The morphology of the respective fibers was imaged by atomic force microscopy.
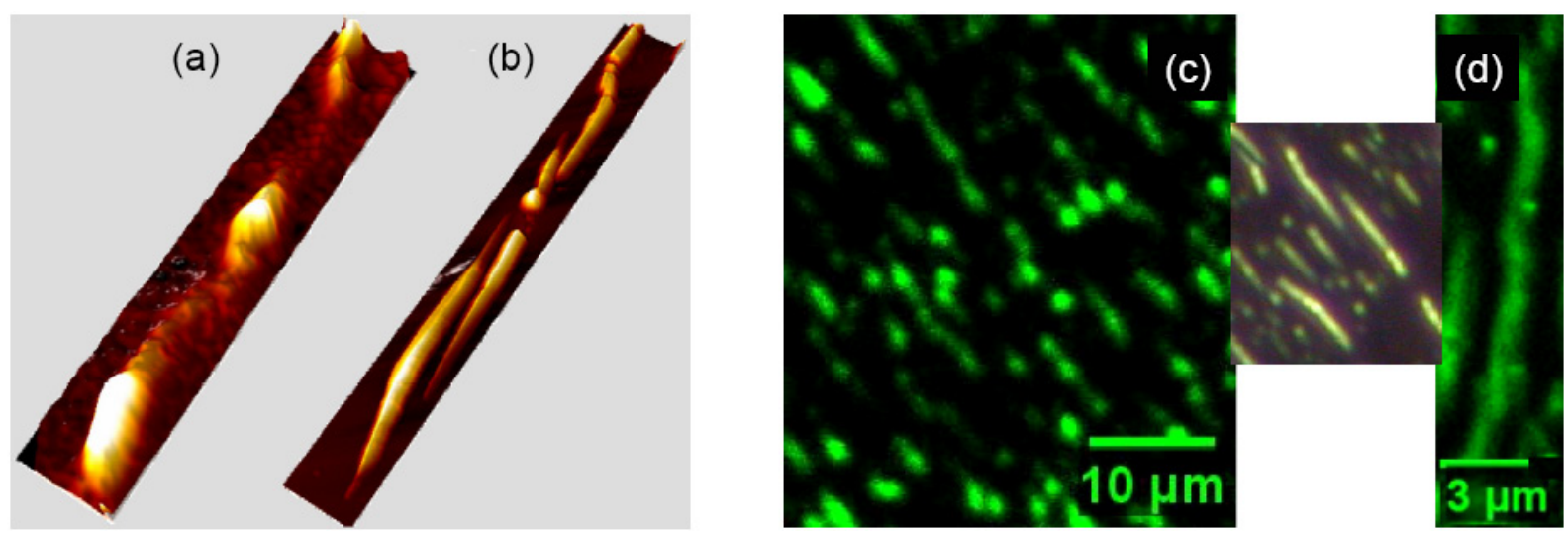

Fig. 6.2: (a) Three-dimensional representation of the measured $\mathrm{SH}$ intensity $(9.8 \times$ $1.8 \mu \mathrm{m}^{2}$, resolution $500 \mathrm{~nm}$ ) from transferred MONHP4 fibers. 'Height scale' refers to relative SH intensity. (b) Three dimensional AFM image of the same fibers (height scale $150 \mathrm{~nm}$ ). From [9]. (c) and (d) Two-dimensional imaging of MONHP4 nanofibers, using a femtosecond confocal microscope. The inset shows a dark field image of the same fibers. From [6].

Upon irradiation with femtosecond pulses, a strong second-harmonic peak was observed, clearly proving true second harmonic generation from the bulk of the fiber [9]. As expected the signal shifts with the excitation wavelength. Fig 6.1 (a) shows the emission of a SH-signal from transferred MONHP4 fibers and for comparison the emission from p6P nanofibers on muscovite mica. Obviously the MONHP4 nanofibers emit a strong SH-signal, whereas the frequency doubled signal originating from the p6P nanofibers cannot even be seen in this graph. Only the fluorescence signal of the p6P fibers based on two-photon absorption is clearly visible.

The SHG intensity was measured as a function of the excitation light's polarisation angle with respect to the fixed transition dipole moment vector of the MONHP4 molecules for an array of nanofibers on the mica substrate. A damped $\sin ^{2}$ course is found (Fig.6.1(b)). The damping is due to bleaching. The $\sin ^{2}$ fit shows the ideal course according to Malus law. The polarisation ratio was defined as:

$$
\left(I_{\max }-I_{\min }\right) /\left(I_{\max }+I_{\min }\right)=0.96 \pm 0.05
$$

This large polarisation ratio of the fibers indicates good alignment of the molecules and thus a high degree of crystallinity of the fibers. This crystallinity is conserved during the transfer process to glass. Thus, the first example of freely transferrable nanofiber frequency doublers from designed organic molecules is given. 
Also measurements on single MONHP4 nanofibers have been performed with the laser scanning microscope (LSM) to give spatially resolved imaging of the SH-intensity combined with morphologically investigations by means of atomic force microscopy. A local variation of the SH intensity in transmission and in reflection has been observed simultaneously. Optically imaged fibers have been marked by ablating patterns around the fibers, which could then be identified in an inverted optical microscope situated below the AFM. Therefore, it was possible to obtain three-dimensional images by LSM and AFM of the very same fiber. The three-dimensional plots for an assembly of three MONHP4 nanofibers are shown in Fig.6.2. Obviously the height profile and the SH intensity profile are not in coincidence. A closer look reveals that the SH intensity increases at inhomogeneous areas along the fiber such as breaks or bends. This means that the SH signal intensity does not simply correlate to the amount of deposited material in contrast to the two-photon luminescence, but depends sensitively on local surface morphological variations. Hence, the generation of the SH signal is not only given by the intrinsic large hyperpolarisability of the molecular building block. Its local variation along individual nanofibers is dominated by local field enhancement of the responsible effective dielectric function of the nanofibers. The local field enhancement, in turn, is due to the local changes in the fibers's morphology (for details see [9, 189]).

Using a laser scanning microscope it is obviously possible to image the nanofibers using second harmonic generation (Fig.6.2(c),(d)). That way SHG imaging is an interesting, label-free new method, which can be used as a complement to traditional fluorescence laser scanning microscopy.

Similar investigations concerning their potential as nanoscaled frequency doublers were performed on nanofibers from two other differently di-functionalised $p$-quaterphenylene oligomers, namely MOCLP4 and MOCNP4, but only for the arrays of nanofibers on their muscovite mica substrate. The basic difference between those and the MONHP4 nanofibers is, that the latter ones can be considered as non-fluorescencent, whereas the nanostructures from MOCLP4 and MOCNP4 emit intense blue fluorescence light.

It has been discussed above that MOCLP4 oligomers self-assemble into two morphologically different nanostructure types depending on the substrate temperature. The low temperature case leads to mutually aligned nanobranches. Upon excitation of these MOCLP4 nanobranches with femtosecond near infrared laser pulses (100 fs) at different wavelengths, they show a pronounced SH signal (Fig6.3(a)). As expected the SH signal shifts with the wavelength of the exciting laser beam. An accompanying fluorescence signal appears for excitation wavelengths shorter than $800 \mathrm{~nm}$. This fluorescence spectrum is the same as obtained after continuous wave excitation with UV light.

The polarisation ratio for MOCLP4 nanofibers was ascertained to be $0.71 \pm 0.05$ according to Equ 6.3, which is significantly lower than in the case of MONHP4 nanofibers. 

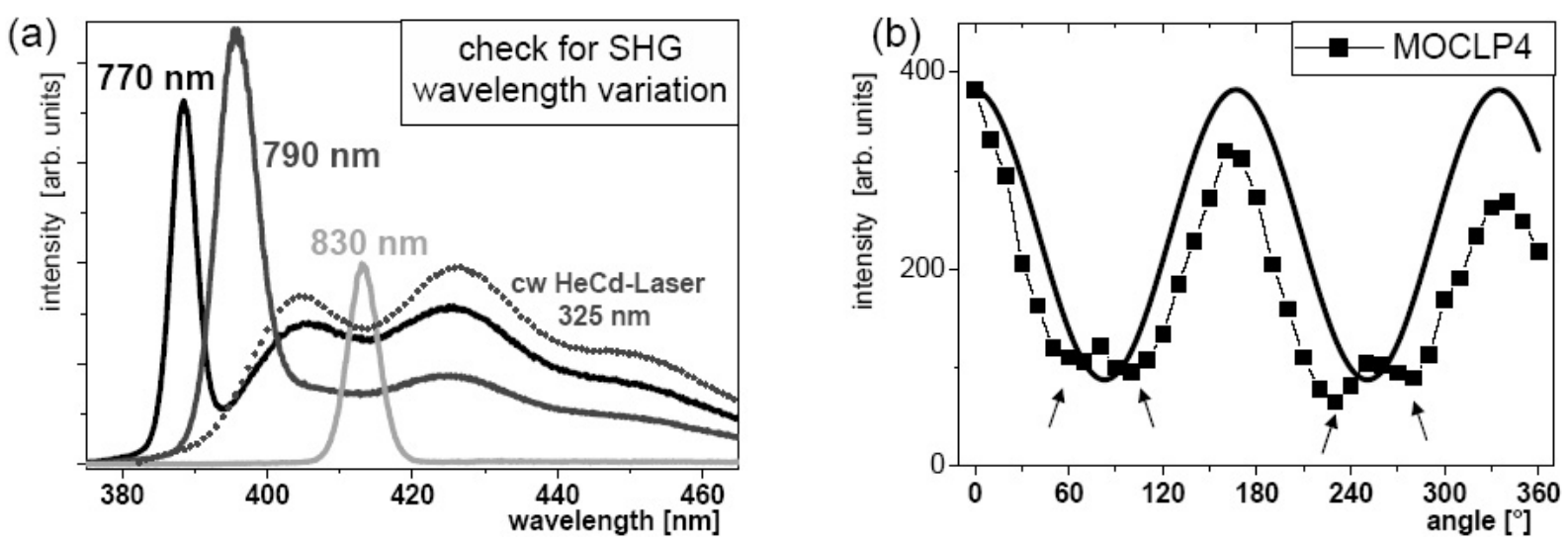

Fig. 6.3: (a) Emission of second harmonic signal from MOCLP4 nanobranches on muscovite mica after excitation with femtosecond near infrared laser pulses (100 fs) at different wavelengths $(770 \mathrm{~nm}, 790 \mathrm{~nm}, 830 \mathrm{~nm}$ ). The fluorescence spectrum after cw UV excitation is shown for comparision as well. (b) Polarisation dependence of the SH-signal from MOCLP4 nanofibers as a function of the rotational angle. Solid line is a $\sin ^{2}$ fit indicating an ideal course without damping. Arrows mark the measured polarisation minima.

This is not necessarly due to a lower degree of crystallinity. The polarisation ratio was measured for an array of nanobranches, which show slightly different growth directions: the part before the fiber splits and the branches, which show no bilateral symmetry, i.e. one branch is significantly longer for most branches (cf. Chapter Growth). The polarisation dependent measurement for the SH signal's intensity reveals four polarisation minima (Fig 6.3(b)). This militates for two different crystallographic orientations of the molecules within the nanoaggregates, each reflected by two different polarisation directions leading to overall four minima for the whole $360^{\circ}$ circle. This in turn is a possible explanation why at the minimum SH intensity is still a certain intensity left: not every SH intensity can be cut out by the polariser at the same time.

For the nanofibers of MOCNP4, the walking sticks, a polarisation ratio of $0.98 \pm 0.05$ can be found (Fig 6.4(b)), which is the highest among the nanostructures from the differently di-functionalised oligomers. Again the damped course of the measured SH intensity is due to bleaching. Upon irriadiation with femtosecond laser pulses at $790 \mathrm{~nm}$ a strong SH signal can be observed (Fig 6.4(a)). The fluorescence is clearly excited as well.

For mono functionalised $p$-quaterphenylenes optical SHG has been recorded after excitation of the arrays of nanofibers on the mica substrate with femtosecond laser pulses within the near infrared. An exemplary spectrum of the SH-signal originating from the nanofibers of CNHP4 after irradiation with 100 fs laser pulses at $826 \mathrm{~nm}$ is shown in Fig 6.5 . For this excitation wavelength the fluorescence is not excited. The nanoaggre- 

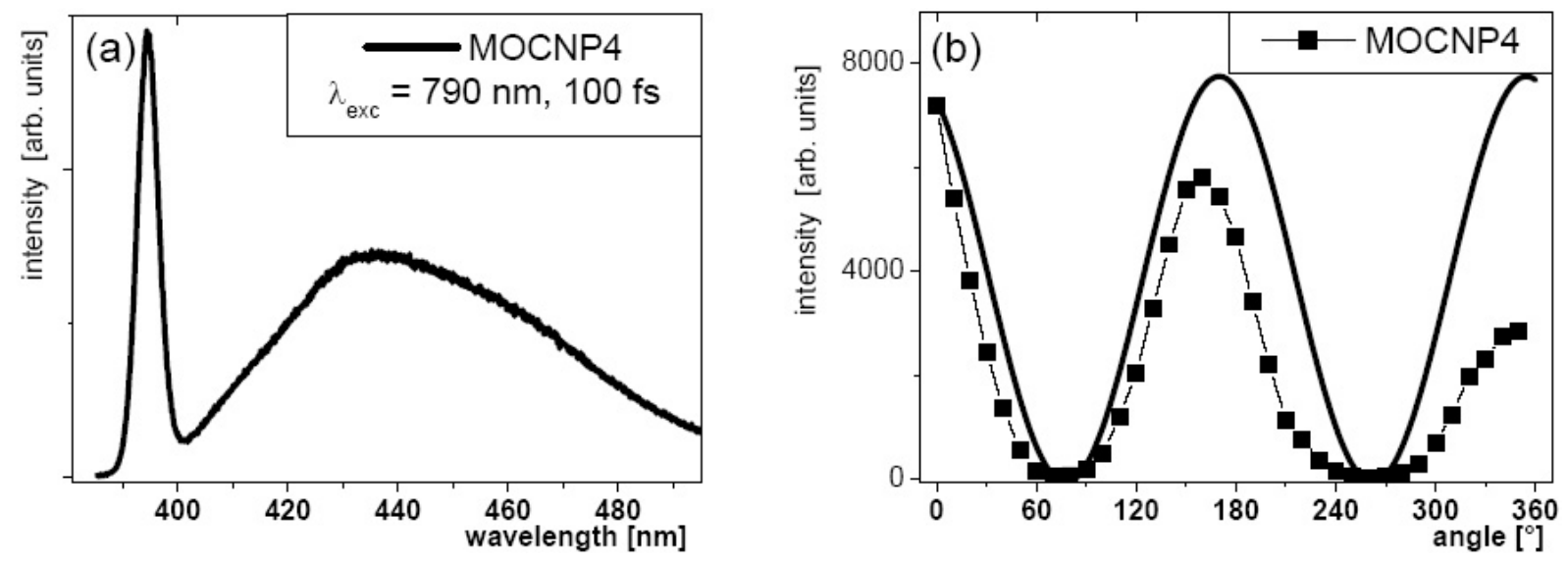

Fig. 6.4: (a) Emission of second harmonic signal from MOCNP4 nanofibers on muscovite mica after excitation with femtosecond near infrared laser pulses (100 fs) at $790 \mathrm{~nm}$. (b) Polarisation dependence of the SH-signal from MOCNP4 nanofibers as a function of the rotational angle. Solid line is a $\sin ^{2}$ fit indicating an ideal course without damping.

gates of all four mono functionalised $p$-quaterphenylenes show clear SH-signals: they act as nanoscaled frequency doublers.

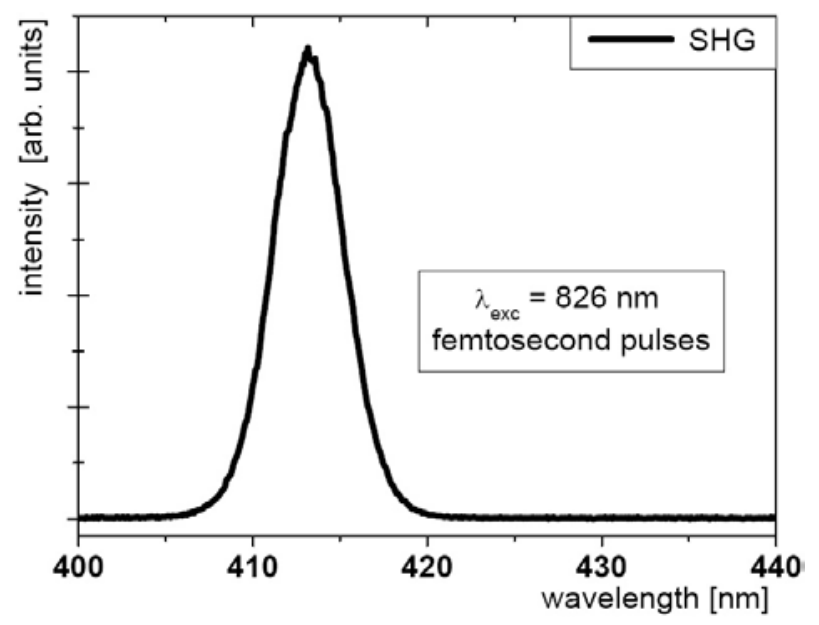

Fig. 6.5: SHG origination from nanofibers of CNHP4 on muscovite mica after excitation with femtosecond laser pulses (100 fs) at $826 \mathrm{~nm}$.

Also a cautious qualitative order of the SHG intensities for the four different oligomers is given. A quantitative comparison of the SH signal's intensities is not possible so far, because the intensity is strongly influenced by local changes of the nanostructure's morphology (see discussion above) and nominal film thicknesses of the sample, and because of difficulties finding a reliable reference for the measurements. Only a careful qualitative comparison can be made. The SH signal's intensities have been measured for arrays of nanostructures on the mica substrate under similar conditions, i.e. same excitation 
wavelength and power and nominal film thicknesses of the samples, which results in the following qualitative order:

$$
\text { CNHP4 > NMeHP4 > MOHP4 > CLHP4 }
$$

The SH-signal from the CNHP4's nanofibers is about 80 times stronger compared to the other nanofibers. Between NMeHP4, MOHP4 and CLHP4 are only minor differences concerning the intensities of the SH-signal.

A similar trend has been found for the differently functionalised oligomers, i.e. the following qualitative order of $\mathrm{SH}$ intensities can be found:

$$
\text { MOCNP4 > MONHP4 > MOCLP4 }
$$

Functionalisation with a cyano group leads to strong SH signals, whereas functionalisation with a chloride group results in significantly lower SH intensities. 
6 Non-linear Optics [6, 9] 


\section{Experimental}

\subsection{Organic Molecular Beam Epitaxy (OMBE)}

Preparation of the organic films takes place in a three-chamber UHV chamber under high vacuum [190]. UHV is possible, but for screening of different oligomers and process parameters high vacuum is sufficient. Fig.7.1 shows schematically the organic molecular beam epitaxy (OMBE) apparatus.

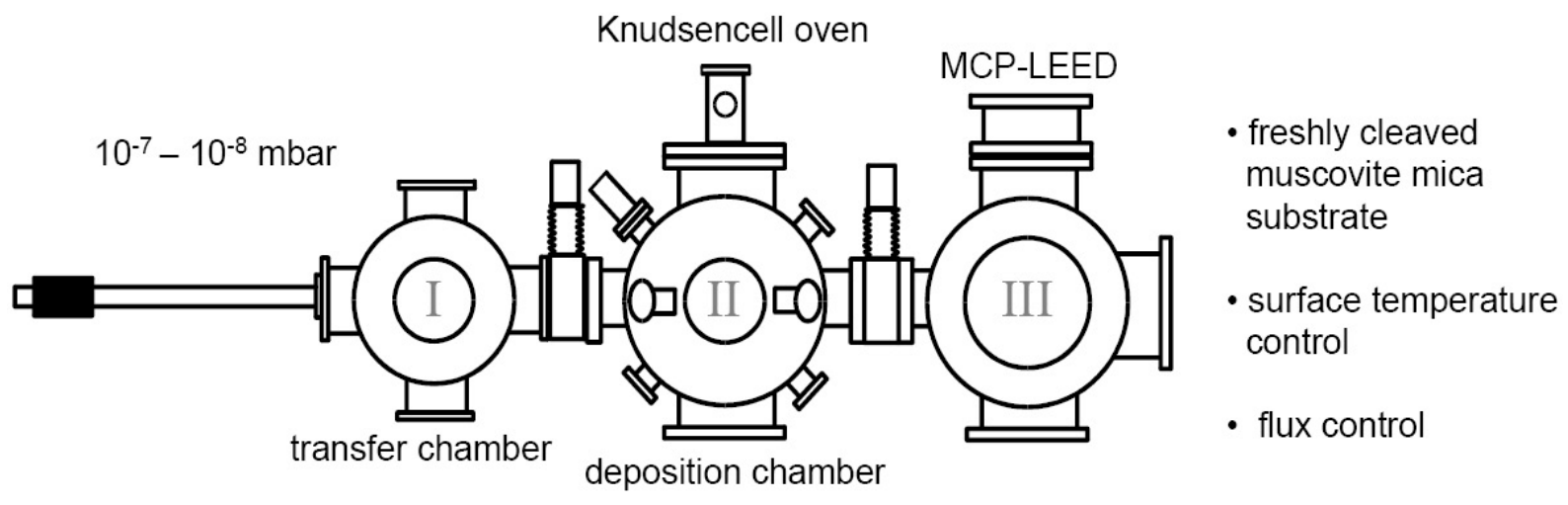

Fig. 7.1: The high vacuum chamber used for OMBE constitutes of three different sections: (I) magnetically coupled transfer system, (II) deposition chamber including a Knudsen sublimation cell and watercooled quartz microbalance system, and (III) characterisation chamber equipped with a MCP-LEED. Pressure as indicated is measured by a Bayard-Alpert ionisation gauges.

First a freshly cleaved muscovite mica sample is introduced into the transfer chamber (I). Without breaking the vacuum samples are moved into the deposition chamber (II) (base pressure $5 \times 10^{-8}$ mbar) using a magnetically coupled transfer system. The organic molecule deposition has been performed via sublimation from a home built Knudsen cell. The deposition rate $(0.1-0.2 \AA / \mathrm{s})$ and the nominal film thicknesses were monitored by a watercooled quarz microbalance (Inficon XTC/2). The mica sample can be heated by a tungsten filament from underneath in a controlled fashion. Since the mica sheet is mounted on a copper plate heating is homogeneous. The sample temperature is determined via the filament current and a previously measured reference curve. A Ni-NiCr thermocouple was clamped on top of a reference mica sheet to allow for its poor thermal conductivity temperature measurement. The third part of the vacuum system (III) 
(base pressure $5 \times 10^{-9}$ mbar) is used for in-situ characterisation of the deposited films by low-energy electron diffraction (LEED). The LEED apparatus (Omicron MCP-LEED) was equipped with two multi-channelplates to limit the electron current density on the substrate and therefore minimize beam damage effects and reduce charging.

Before deposition the molecules were kept in high vacuum for at least eight hours and heated just below the sublimation temperature for half an hour in order to outgas and to remove residual organic solvents and water. The pressure during deposition increased to about $8 \times 10^{-7}$ mbar. As growth substrate freshly cleaved muscovite mica (grade V-4 from Structure Probe, Inc.) was used. After immediate transfer into the high vacuum chamber, the mica substrate was heated for at least two hours for outgassing purposes.

\subsection{Epifluorescence and Darkfield Microscopy}

An optical microscope is an instrument for viewing objects, which are too small to be seen by the naked eye, by using light and a system of magnifying lenses. They are the most widely used types of microscopes and trace their history almost back for 400 years. In context of this thesis a Nikon eclipse microscope is utilised for imaging samples by epifluorescence or sometimes in darkfield illumination technique.
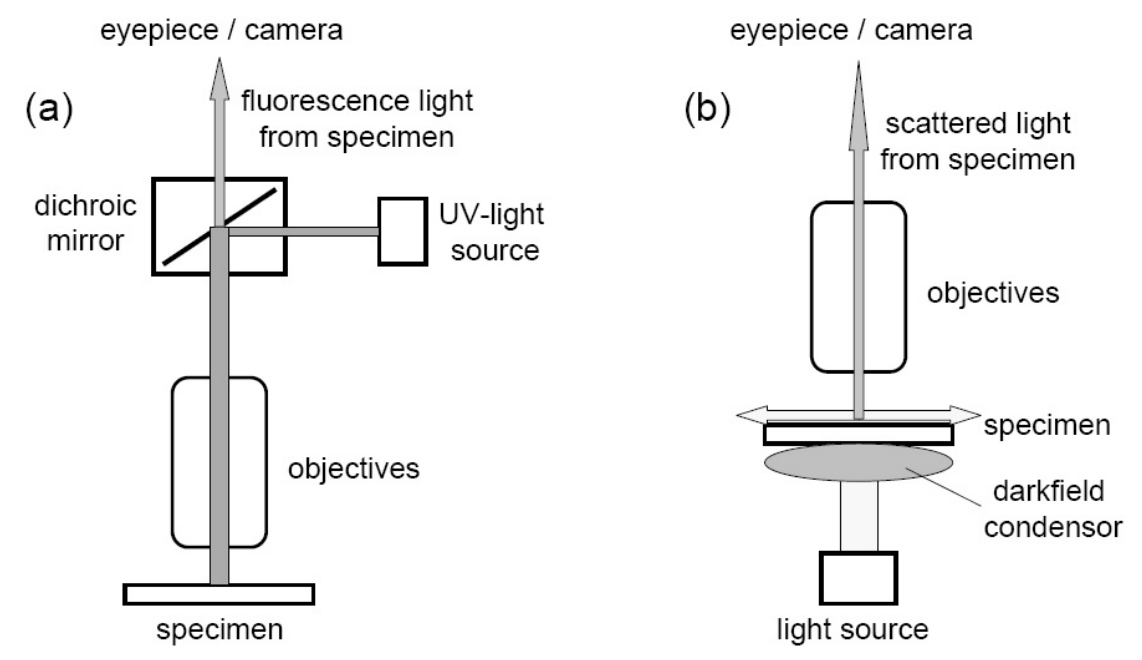

Fig. 7.2: Sketches of (a) epifluorescence microscopy and (b) darkfield microscopy.

To obtain an epifluorescence microscopy image the specimen needs to be fluorescent. It is irradiated with UV-light $\left(\lambda_{\max } \approx 365 \mathrm{~nm}\right)$ from a high pressure mercury lamp. The emitted fluorescence light is collected by an magnifying objective and the illumination light is cut off by a dichroic mirror, Fig.7.2(a). Since most of the nanofibers show bright fluorescence they are ideal specimen and give respectable images. Non-fluorescent samples are imaged by use of a darkfield condensor. The objective does not collect the light from the light source, a halogen lamp, but only scattered light from the features of the sample, 
Fig $7.2(\mathrm{~b})$. This produces a dark background and enhances the contrast of the image compared to conventional bright field. For both ilumination techniques the images can by viewed through an eypiece or pictures can by taken by a digital camera.

The overall magnification is the product of the magnifications from the objective and the ocular (eyepiece). It is up to $1000 \times$ for the used microscope. The resolution of optical microscopes is in general given by the Abbe-limit. It roughly says that features have to be in a distance of more than $d=\lambda / 2$ to be resolved as distinct features, whereas $\lambda$ is the wavelength of the light emitted by the specimen, here $\approx 200 \mathrm{~nm}$.

Additionally, the microscope is equipped with filters to reduce the intensity of the illuminating UV-light and with a polariser to perform polarisation depending measurements of the emitted fluorescence light (see below).

\subsection{Atomic Force Microscopy}

Atomic Force Microscopy (AFM) - invented 1986 by Binning, Quate and Gerber [191] - is very different from optical microscopy. There is no need for an illuminating light source, no lenses and no eyepiece to look through. The imaging technique consists of a mechanical device, which measures small forces between probe and sample. An atomic force microscope is one of the many possible scanning probe microscopes, which is widely used [192]. For this thesis the JPK NanoWizard was used.
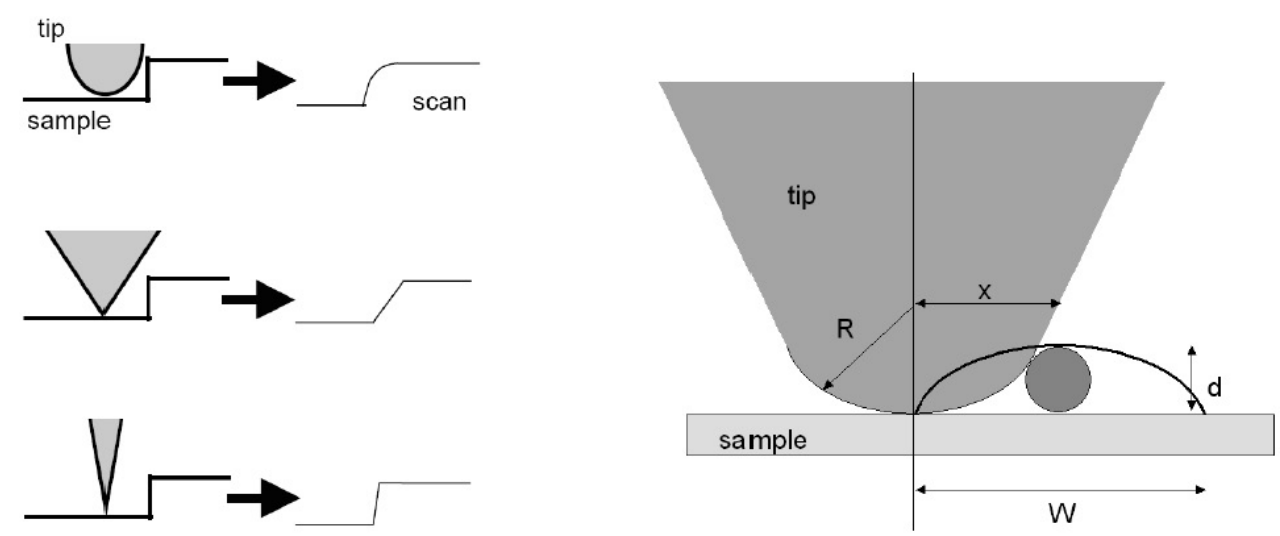

Fig. 7.3: Tip shape issues: Effect of the tip shape on the images (left hand side) and relationship between observed width $\mathrm{W}$ and the diameter of the probe tip (right hand side). See text for further explanations. From [193].

It utilises a flexible silicon cantilever as a type of spring to measure the force between a sharp tip and the sample. The local attractive and repulsive force is converted into a bending, or deflection, of the cantilever. The deflection is measured by a laser beam that is reflected from the back of the cantilever onto a detector. A photodetector is used, which converts the light signal into an electrical signal to produce the image. As the cantilever 


\section{Experimental}

deflects, the angle of the reflected laser beam changes, and the spot falls on different parts of the photodetector. The signals from the four quadrants of the detector are compared to calculate the deflection signal, see Fig.7.4. This signal refers to the topography of the sample when the tip is scanned over the surface. The resolution is given by the quality of the tip, mainly by its sharpness. It can be manufactured with an end radius of a few nanometer. Images show artefacts if the tip is damaged or contaminated. The image is often some combination of the tip shape and the true surface topography, Fig 7.3 . The scheme on the left hand side gives an impression of how the tip shape influences the image of a given feature on the sample, here a perfect rectangular step on the surface. None of the tips shown really produce an exact image of the feature. The sharpest, narrowest tip produces the most accurate representation of the surface. For measuring very narrow structures (below $10 \mathrm{~nm}$ ) the width of the feature is observed significantly wider than it is, Fig.7.4. The relationship between the observed width $\mathrm{W}$ of a feature and the diameter of the probe tip can be calculated for an idealised tip shape, such as shown on the right hand side. For $R>>d, W=\sqrt{8 d R}$. The observed width would be $\mathrm{W}=$ $20 \mathrm{~nm}$ for $\mathrm{R}=10 \mathrm{~nm}$ and $\mathrm{d}=5 \mathrm{~nm}$. This lateral resolution is lower than the resolution of the structures' heights, which can be measured to an accuracy of single nanometers. In general an AFM provides significantly higher resolution than an optical microscope and shows the topography. Therefore it is used for detailed analysis of the nanofiber's morphology.

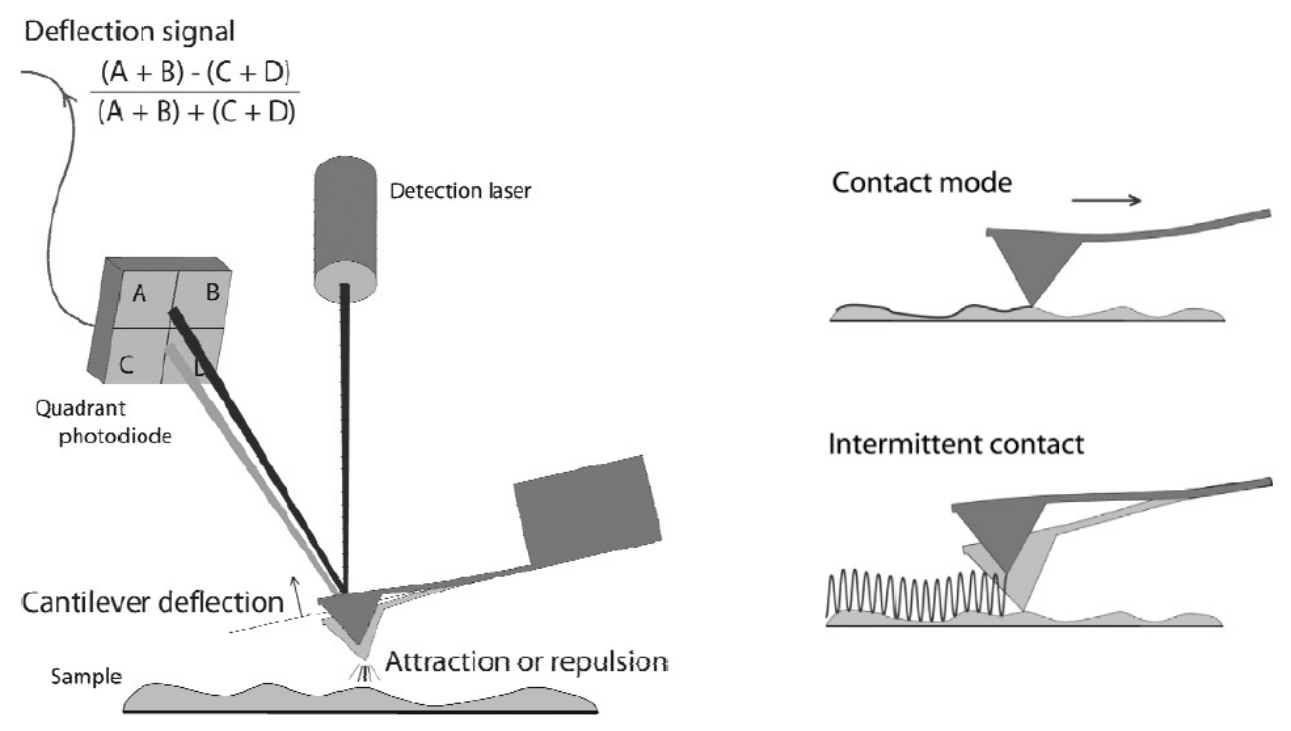

Fig. 7.4: Sketch of an atomic force microscope (left hand side). Basic operation modes are contact mode and intermittent contact mode (right hand side). From [193].

An AFM has two basic operation modes (cf. Fig.7.4): (I) In the contact mode the tip never leaves the surface, so it can be used for very high resolution imaging down to atomic resolution of e.g. inorganic crystals. (II) In intermittent contact mode the tip is 
oscillating perpendicular to the surface while moving over the sample. Since the lateral forces are much lower than in contact mode, it can be used for imaging soft samples, which have objects not firmly stuck to the surface without moving them around. Thus, the intermittent contact mode is favourable for organic nanoaggregates on a mica substrate and is used for all AFM imaging in this thesis. The cantilever is driven close to a resonance of the system to give reasonable amplitude for the oscillation and also phase information. The resonant frequency of the cantilever depends on its mass and spring constant; stiffer cantilevers have higher resonant frequencies. Typically they are in the range of $200-400$ $\mathrm{kHz}$ with spring constants more than $10 \mathrm{~N} / \mathrm{m}$ for the use in air.

\subsection{Measurement of optical properties}

Fluorescence spectra have been recorded after continous wave (cw) UV excitation of the aggregates with a He:Cd laser at an emission wavelength of $325 \mathrm{~nm}$ on the mica substrate, Fig.7.5. Irradiation took place at the top side of the sample in this case to avoid absorption of UV light from the mica substrate. The emission light from the nanofibers was sent through lenses to a cooled CCD spectrometer (resolution $1 \mathrm{~nm}$ ).

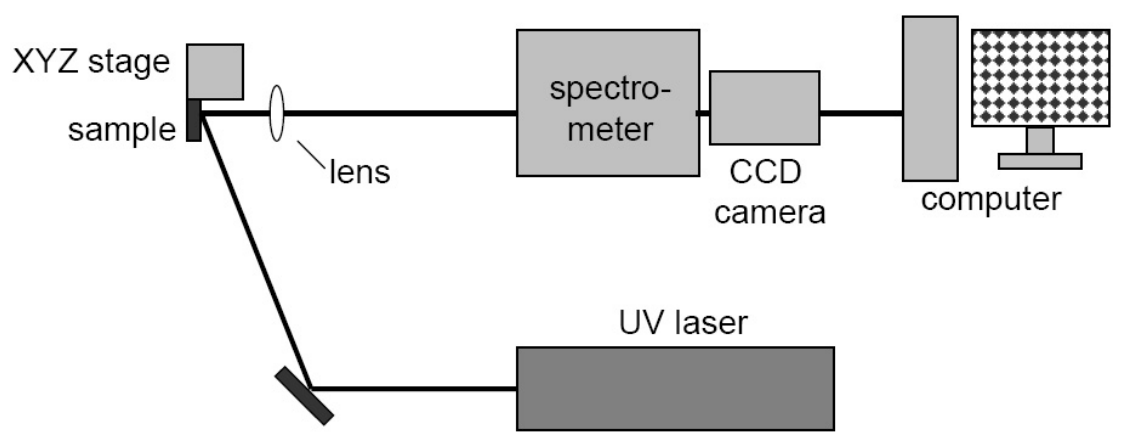

Fig. 7.5: Sketch of experimental setup for measurement of fluorescence spectra.

For measurements of SHG optical spectra a Spectra Physics Tsunami femtosecond resonator was used to excite the nanofibers at wavelengths between $750 \mathrm{~nm}$ and $850 \mathrm{~nm}$. The resulting light emission from the fibers was sent through a series of lenses and bandpass filters to a cooled CCD spectrometer (resolution $1 \mathrm{~nm}$ ), Fig.7.6(a). For imaging and investigations of the SHG as a function of excitation wavelength a laser scanning microscope (LSM) was used. A laser scanning confocal microscope (Zeiss LSM 510 META) coupled with a Spectra Physics Mai-Tai (wideband, 710-990 nm) Ti:sapphire laser for two photon excitation experiments served to obtain three-dimensional images of individual needles. The excitation power of the laser at different wavelengths was measured directly at the sample position so as to know the real excitation power at the sample. The SHG signal from the sample could be recorded in transmission with a photomultiplier (PM) above 
the sample and in reflection with a photomultiplier (PM) below the sample, Fig. $7.6(\mathrm{~b}) .1$
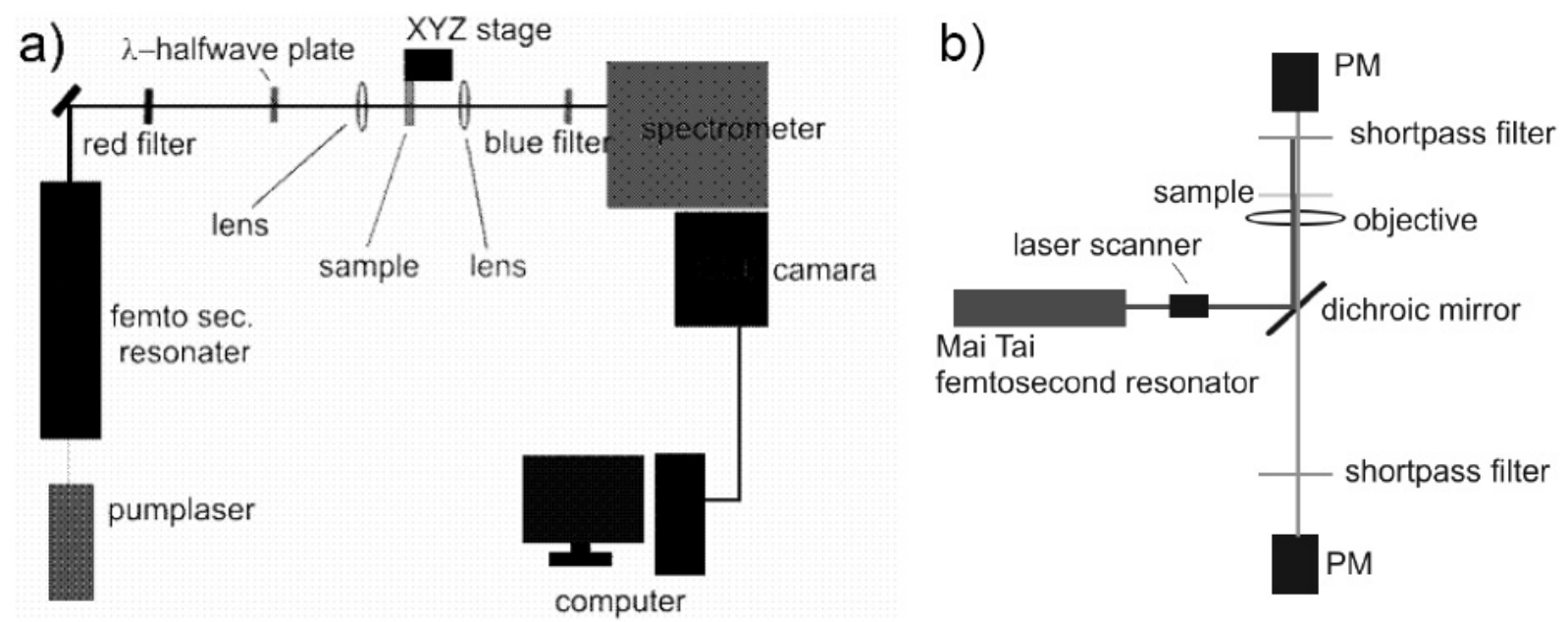

Fig. 7.6: (a) Sketch of experimental setup for measurement of SHG optical spectra. (b) Schematics of the LSM setup. From [188].

Measurements of polarisation dependence have been performed in a fluorescene microscope (see above). The samples have been irradiated with unpolarised UV light from a high pressure mercury lamp $\left(\lambda_{e x c}=365 \mathrm{~nm}\right)$. Pictures were taken through a polariser with a CCD camera as a function of the polarisation angle ( $5^{\circ}$-steps). Evaluation of this picture series leads to polarisation properties with respect to fiber orientations. The angular distribution of fluorescence can be fit by Malus law. It says that when a perfect polariser is placed in a linearly polarised beam of light, the intensity, $I$, of the light that passes through is given by

$$
I=I_{0} \cdot \cos ^{2} \theta_{i}
$$

where $I_{0}$ is the initial intensity, and $\theta_{i}$ is the angle between the light's initial plane of polarisation and the transmission axis of the polariser. Here the maxiumum intensity of light indicates the polarisation direction of the nanofibers' fluorescence.

\subsection{Further Characterisation Methods}

- UV/Vis absorption spectra in solution have been measured on a Jena Analytic Specord 200 spectrometer in a $1 \mathrm{~cm}$ quartz cuvette.

- Fluorescence spectra in solution have been recorded on an Amico Bowman AB2 spectrometer in a $1 \mathrm{~cm}$ quartz cuvette. ${ }^{2}$

\footnotetext{
${ }^{1}$ Jonathan Brewer is thanked for setup and performance of the SHG measurements.

${ }^{2}$ Ivonne Wallmann, University of Bonn is thanked for the measurements.
} 
- ${ }^{1} \mathrm{H}$ - and ${ }^{13} \mathrm{C}-\mathrm{NMR}$ spectra were recorded at $300 \mathrm{~K}$ in $\mathrm{CDCl}_{3}$ solutions on a Bruker Avance 500 spectrometer at 500.1 and $125.8 \mathrm{MHz}$, respectively. ${ }^{1} \mathrm{H}-\mathrm{NMR}$ chemical shifts are reported on the $\delta$-scale in ppm relative to residual nondeuterated solvent as internal standard. ${ }^{13} \mathrm{C}$-NMR chemical shifts are reported on the $\delta$-scale in ppm relative to deuterated solvent as internal standard 3

- The mass spectra were taken on a Finnigan MAT 95 with data system DEC-Station 5000 (EI, HiRes-EI, CI, HiRes-CI, isobutane or $\left.\mathrm{NH}_{3}\right)\left.\cdot\right|^{4}$

\footnotetext{
${ }^{3}$ Central Analytics, University of Oldenburg, D. Neemeyer and M. Rundshagen are thanked for the measurements.

${ }^{4}$ Central Analytics, University of Oldenburg, F. Fabretti is thanked for the measurements.
} 


\subsection{Organic Synthesis}

\subsubsection{General remarks}

4,4,5,5-tetramethyl-1,3,2-dioxaborolane, (4-bromophenyl)boronic acid, (4-chlorophenyl)boronic acid, (4-methoxyphenyl)boronic acid, (4-nitrophenyl)boronic acid, (4-cyanophenyl)boronic acid, tert-butyl-4-(4,4,5,5-tetramethyl-1,3,2-dioxaborolan-2-yl)- phenylcarbamate, 1-(diphenylphosphino)-2-(2-(diphenylphosphino)phenoxy)benzene, 4,4'- biphenyldiboronic acid bis(neopentyl glycol)ester, 4-,4'-dibromobiphenyl, 4-iodonitrobenzene, cesium fluoride, tetrakis(triphenylphosphino)palladium $(0)\left(\left[\mathrm{Pd}\left(\mathrm{PPh}_{3}\right)_{4}\right]\right)$, and palladium(II)acetate $\left(\mathrm{Pd}(\mathrm{OAc})_{2}\right)$ were purchased from ABCR, Sigma-Aldrich, or Atlantic Scientific Co. and used as received. Bis(triphenylphosphino)palladium(II)-chloride $\left.\left(\mathrm{Pd}\left(\mathrm{PPh}_{3}\right)_{2} \mathrm{Cl}_{2}\right)\right)$ was prepared following a published procedure [194]. Most solvents were dried, distilled and stored under argon according to standard procedures. Reactions with air- and moisture-sensitive transition metal compounds were performed under an argon atmosphere in oven-dried glassware using standard Schlenk techniques. Thin-layer chromatography was performed on aluminum TLC-plates (silica gel $60 F_{254}$ ) from Merck. Detection was done by UV-light (254 or $366 \mathrm{~nm}$ ). Products were purified by column chromatography on silica gel 60 (70-230 mesh or 230-400 mesh) from Merck or by extraction and washing procedures. Since all of the $p$-quaterphenylenes were obtained as amorphous solids they generally did not give sufficient elemental analyses due to residual amounts of solvents within the powder-like solids. These could not be completely removed even after heating for longer periods of time in standard laboratory high vacuum $\left(10^{-3} \mathrm{mbar}\right)$. Thus high-resolution MS data are given instead.

\subsubsection{Symmetrically functionalised para-Quaterphenylenes}

\section{$1,4^{\prime \prime}$ '-Dimethoxy-4,1':4',1' ':4' ', 1' ' '-quaterphenylene (MOP4) [112, 195-197]}

Under an argon atmosphere $1.1 \mathrm{~g}(3.4 \mathrm{mmol})$ of 4,4'-dibromobiphenyl, $1.14 \mathrm{~g}$ (7.48 mmol, 2.2 equivalents) of 4-methoxyphenylboronic acid, $1.93 \mathrm{~g}$ (12.68 mmol, 4 equivalents) of cesium fluoride, and $110 \mathrm{mg}(0.10 \mathrm{mmol}, 3 \mathrm{~mol} \%)$ of $\left[\mathrm{Pd}\left(\mathrm{PPh}_{3}\right)_{4}\right]$ were dissolved in 60 $\mathrm{mL}$ of abs. THF and refluxed for 50 hours. The product precipitated from the reaction mixture and was collected by filtration. The crude product was washed repeatedly with dichloromethane and water to give $1.26 \mathrm{~g}$ (3.4 mmol, quant.) of MOP4 as a colourless amorphous solid.

${ }^{1} \mathrm{H}-\mathrm{NMR}\left(\mathrm{CDCl}_{3}, \delta[\mathrm{ppm}]\right): 3.86(6 \mathrm{H}, \mathrm{s}), 6.99(4 \mathrm{H}, \mathrm{d}, J=8.5 \mathrm{~Hz}), 7.58(4 \mathrm{H}, \mathrm{d}, J=$ $8.5 \mathrm{~Hz}), 7.64(4 \mathrm{H}, \mathrm{d}, J=8.3 \mathrm{~Hz}), 7.69(4 \mathrm{H}, \mathrm{d}, J=8.3 \mathrm{~Hz})$.

MS (CI, isobutane): $\mathrm{m} / \mathrm{z} 367.1\left(\mathrm{M}+\mathrm{H}^{+}\right)$. 


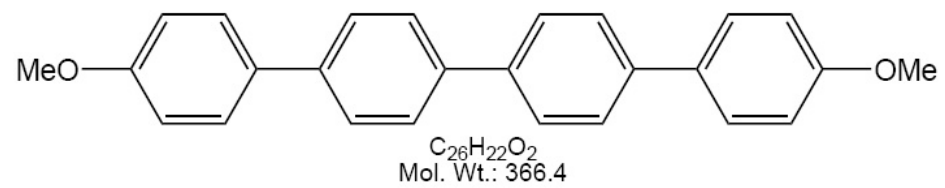

HR-MS (EI): calcd. for $\mathrm{C}_{26} \mathrm{H}_{22} \mathrm{O}_{2}$ : 366.1620; found: 366.1622 .

UVvis (dichloromethane): $\lambda_{\max }=310 \mathrm{~nm}$.

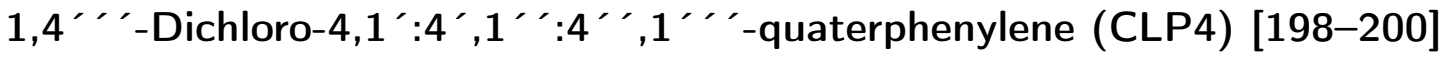

Under an argon atmosphere $631 \mathrm{mg}(2.0 \mathrm{mmol})$ of 4,4'-dibromobiphenyl, $655 \mathrm{mg}$ (4.2 mmol, 2.1 equivalents) of 4-chlorophenylboronic acid, $1.22 \mathrm{~g}$ ( $8.0 \mathrm{mmol}, 4$ equivalents) of cesium fluoride, and $110 \mathrm{mg}(0.10 \mathrm{mmol}, 5 \mathrm{~mol} \%)$ of $\left[\mathrm{Pd}\left(\mathrm{PPh}_{3}\right)_{4}\right]$ were dissolved in 60 $\mathrm{mL}$ of abs. THF and refluxed for 50 hours. The product precipitated from the reaction mixture and was collected by filtration. The crude product was washed repeatedly with dichloromethane and water to give $690 \mathrm{mg}$ (1.84 mmol, 92\%) of CLP4 as a colourless amorphous solid.

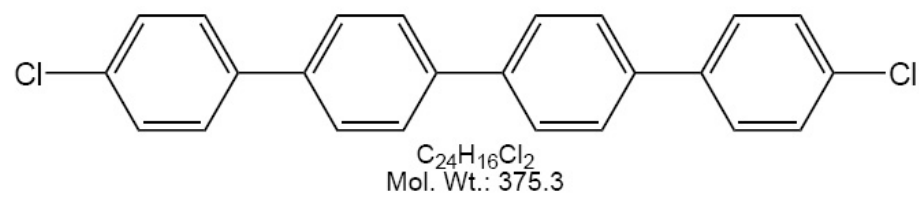

${ }^{1} \mathrm{H}-\mathrm{NMR}\left(\mathrm{CDCl}_{3}, \delta[\mathrm{ppm}]\right): 7.42(4 \mathrm{H}, \mathrm{d}, J=8.4 \mathrm{~Hz}), 7.57(4 \mathrm{H}, \mathrm{d}, J=8.4 \mathrm{~Hz}), 7.64(4$ $\mathrm{H}, \mathrm{d}, J=8.1 \mathrm{~Hz}), 7.71(4 \mathrm{H}, \mathrm{d}, J=8.1 \mathrm{~Hz})$.

MS (CI, isobutane): $\mathrm{m} / \mathrm{z} 375.1\left(\mathrm{M}+\mathrm{H}^{+}\right)$.

HR-MS (EI): calcd. for $\mathrm{C}_{24} \mathrm{H}_{16} \mathrm{Cl}_{2}$ : 374.0629; found: 366.0623 .

UVvis (dichloromethane): $\lambda_{\max }=304 \mathrm{~nm}$.

\section{$1,4^{\prime \prime} '$-Dicyano-4, $41^{\prime}: 4^{\prime}, 1^{\prime \prime}: 4{ }^{\prime \prime}, 11^{\prime \prime \prime}$-quaterphenylene (CNP4)}

Under an argon atmosphere $452 \mathrm{mg}$ (1.45 mmol) of 4,4'-dibromobiphenyl, $450 \mathrm{mg}$ (3.05 mmol, 2.1 equivalents) of 4-cyano phenyl boronic acid, $881 \mathrm{mg}$ (5.8 mmol, 4 equivalents) of cesium fluoride, and $84 \mathrm{mg}(0.07 \mathrm{mmol}, 5 \mathrm{~mol} \%)$ of $\left[\mathrm{Pd}\left(\mathrm{PPh} 4_{3}\right)_{4}\right]$ were dissolved in $50 \mathrm{~mL}$ of abs. THF and refluxed for 50 hours. After cooling to room temperature the reaction mixture was diluted with n-hexane and water. The precipitate was collected by filtration and washed repeatedly with ethylacetate and water for purification to give 421 mg (1.18 mmol, 81\%) of CNP4 as a colourless amorphous solid.

${ }^{1} \mathrm{H}-\mathrm{NMR}\left(\mathrm{CDCl}_{3}, \delta[\mathrm{ppm}]\right): 7.70(4 \mathrm{H}, \mathrm{d}, J=8.2 \mathrm{~Hz}, \mathrm{H}-7), 7.72-7.77$ (12 H, m, H-3, $\mathrm{H}-4, \mathrm{H}-8)$.

${ }^{13} \mathrm{C}-\mathrm{NMR}\left(\mathrm{CDCl}_{3}, \delta[\mathrm{ppm}]\right): 111.1(\mathrm{C}-2), 118.8(\mathrm{C}-1), 127.6\left(\mathrm{C}^{*}-4\right), 127.7\left(\mathrm{C}^{*}-7, \mathrm{C}^{*}-8\right)$, 132.6 (C-3), $138.4\left(\mathrm{C}^{*}-6\right), 140.5\left(\mathrm{C}^{*}-9\right), 144.9(\mathrm{C}-5)$. 


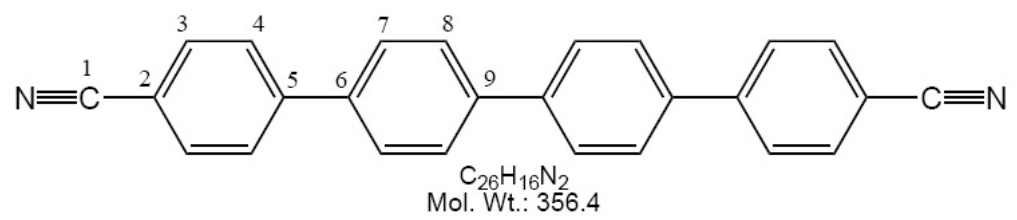

MS (CI, isobutane): $\mathrm{m} / \mathrm{z} 357.4\left(\mathrm{M}+\mathrm{H}^{+}\right)$.

HR-MS (EI): calcd. for $\mathrm{C}_{26} \mathrm{H}_{16} \mathrm{~N}_{2}$ : 356.1313; found: 356.1312 .

UVvis (dichloromethane): $\lambda_{\max }=303 \mathrm{~nm}$.

\section{$1,4^{\prime}$ ' '-Di- N,N-dimethylamino-4, $1^{\prime}: 4^{\prime}, 1^{\prime}{ }^{\prime}: 4^{\prime \prime}, 1^{\prime}$ ' ' $^{\prime}$-quaterphenylene (NMeP4) [201]}

Under an argon atmosphere $567 \mathrm{mg}(1.50 \mathrm{mmol})$ of 4,4'-biphenyldiboronic acid bis(neopentyl glycol)ester, $549 \mathrm{mg}$ (3.19 mmol, 2.2 equivalents) of 4-bromo-N,N-dimethyl aniline, $684 \mathrm{mg}$ ( $4.5 \mathrm{mmol}, 3$ equivalents) of cesium fluoride, and $87 \mathrm{mg}$ (0.075 mmol, 5 $\mathrm{mol} \%)$ of $\left[\mathrm{Pd}\left(\mathrm{PPh}_{3}\right)_{4}\right]$ were dissolved in $60 \mathrm{~mL}$ of abs. THF and refluxed for 50 hours. The grey precipitate was filtered off, washed with tetrahydrofurane and water. After suspending the precipitate in about $100 \mathrm{~mL}$ of dichloromethane, trifluoroacetic acid was added in small portions and the remaining grey-black residue was removed by filtration. Upon adding of small portions of $10 \mathrm{M}$ aqueous sodium hydroxide to the filtrate the crude product precipitated and was collected by filtration. It was washed repeatedly with dichloromethane and water to give $569 \mathrm{mg}$ (1.45 mmol, 96\%) of NMeP4 as a light yellow amorphous solid that turned out to be slightly contaminated with a byproduct. For further purification the crude product was dissolved in about $100 \mathrm{~mL}$ of dichloromethane containing a few $\mathrm{mL}$ of trifluoroacetid acid. Then about $100 \mathrm{~mL}$ of $n$-hexane were added to give a colourless precipitate. After 2 hours of sedimentation the precipitate was collected by filtration, washed repeatedly with dichloromethane and water to give $318 \mathrm{mg}$ (0.81 mmol, $54 \%)$ of 7 as a colourless amorphous solid.

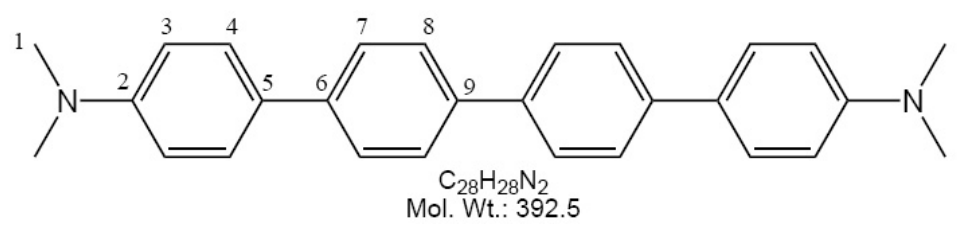

${ }^{1} \mathrm{H}-\mathrm{NMR}\left(\mathrm{CDCl}_{3}:\right.$ trifluoroacetic acid 3:1, $\left.\delta[\mathrm{ppm}]\right): 3.40(6 \mathrm{H}, \mathrm{s}, \mathrm{H}-1), 7.58(4 \mathrm{H}, \mathrm{d}, J=$ $8.6 \mathrm{~Hz}, \mathrm{H}-4), 7.68(4 \mathrm{H}, \mathrm{d}, J=8.2 \mathrm{~Hz}, \mathrm{H}-8), 7.78(4 \mathrm{H}, \mathrm{d}, J=8.2 \mathrm{~Hz}, \mathrm{H}-7), 7.84(4 \mathrm{H}$, $\mathrm{d}, J=8.6 \mathrm{~Hz}, \mathrm{H}-3), 10.0\left(2 \mathrm{H}\right.$, broad s, protonated $\left.-N M e_{2}\right)$.

${ }^{13} \mathrm{C}-\mathrm{NMR}\left(\mathrm{CDCl}_{3}\right.$ :trifluoroacetic acid 3:1, $\left.\delta[\mathrm{ppm}]\right): 120.2(\mathrm{C}-4), 127.8(\mathrm{C}-8), 127.9(\mathrm{C}-7)$, 129.6 (C-3), 137.8 (C-5), 140.6 (C-2), 140.8 (C-9), 144.3 (C-6).

MS (EI): m/z $392.2\left(\mathrm{M}^{+}\right)$. 
HR-MS (EI): calcd. for $\mathrm{C}_{28} \mathrm{H}_{28} \mathrm{~N}_{2}$ : 392.2252; found: 392.2251 .

UVvis (dichloromethane:trifluoreoacetic acid 10:1): $\lambda_{\max }=303 \mathrm{~nm}$.

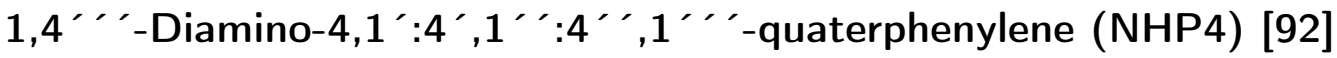

Under an argon atmosphere $308 \mathrm{mg}(1.0 \mathrm{mmol})$ of 4,4'-dibromo biphenyl, $668 \mathrm{mg}$ (2.1 mmol, 2.1 equivalents) of tert-butyl- 4-(4,4,5,5-tetramethyl-1,3,2-dioxaborolan-2-yl)phenylcarbamate, $608 \mathrm{mg}$ ( $4.0 \mathrm{mmol}, 4$ equivalents) of cesium fluoride, and $56 \mathrm{mg}$ (0.05 mmol, $5 \mathrm{~mol} \%)$ of $\left[\mathrm{Pd}\left(\mathrm{PPh}_{3}\right)_{4}\right]$ are dissolved in $40 \mathrm{~mL}$ of abs. THF and refluxed for 50 hours. The precipitate was collected by filtration and washed repeatedly with tetrahydrofurane and water to give a light yellow amorphous solid. This was suspended in dichloromethane and cooled to $0{ }^{\circ} \mathrm{C}$. Then $12 \mathrm{~mL}$ of trifluoroacetic acid were added dropwise and the resulting solution was stirred for 6 hours at room temperature. After removing of some grey insoluble residues by filtration, the solution was made alkaline by adding $20 \mathrm{~mL}$ of $2 \mathrm{M}$ aqueous sodium hydroxide dropwise to precipitate the product from the reaction mixture. The residue was collected by filtration and washed repeatedly with water and dichloromethane to give $310 \mathrm{mg}$ (0.92 mmol, 92\%) of NHP4 as a slightly coloured amorphous solid.

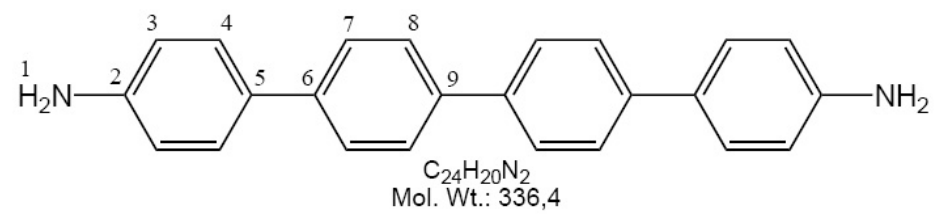

${ }^{1} \mathrm{H}-\mathrm{NMR}\left(\mathrm{CDCl}_{3}\right.$ :trifluoroacetic acid 3:1, $\left.\delta[\mathrm{ppm}]\right): 7.47(4 \mathrm{H}, \mathrm{d}, J=8.3 \mathrm{~Hz}, \mathrm{H}-4), 7.68$ $(4 \mathrm{H}, \mathrm{d}, J=8.3 \mathrm{~Hz}, \mathrm{H}-8), 7.76(4 \mathrm{H}, \mathrm{d}, J=8.3 \mathrm{~Hz}, \mathrm{H}-7), 7.79(4 \mathrm{H}, \mathrm{d}, J=8.3 \mathrm{~Hz}, \mathrm{H}-3)$, 8.77 (6 H, broad s, H-1).

${ }^{13} \mathrm{C}-\mathrm{NMR}\left(\mathrm{CDCl}_{3}\right.$ :trifluoroacetic acid 3:1, $\left.\delta[\mathrm{ppm}]\right): 123.1(\mathrm{C}-4), 127.2(\mathrm{C}-2), 127.8\left(\mathrm{C}_{-}\right.$ 8), 127.9 (C*-7), 129.2 (C-3), 138.2 (C-5), 140.6 (C-9), 143.6 (C-6).

UVvis (dichloromethane): $\lambda_{\max }=324 \mathrm{~nm}$.

UVvis (dichloromethane:trifluoreoacetic acid 10:1): $\lambda_{\max }=300 \mathrm{~nm}$.

No mass spectra could be obtained.

\section{$1,4^{\prime \prime}$ '-Dinitro-4,1' $: 4^{\prime}, 1^{\prime \prime}: 4^{\prime \prime}, 1^{\prime \prime}{ }^{\prime}$ 'quaterphenylene (NOP4) [92, 112, 202]}

Under an argon atmosphere $378 \mathrm{mg}(1.00 \mathrm{mmol})$ of 4,4'-biphenyldiboronic acid bis(neopentylglycol)ester, $548 \mathrm{mg}$ (2.2 mmol, 2.2 equivalents) of 4-iodo-nitrobenzene, 608 $\mathrm{mg}$ (4.08 mmol, 4 equivalents) of cesium fluoride, and $58 \mathrm{mg}(0.05 \mathrm{mmol}, 5 \mathrm{~mol} \%)$ of $\left[\mathrm{Pd}\left(\mathrm{PPh}_{3}\right)_{4}\right]$ were dissolved in $50 \mathrm{~mL}$ of abs. THF and refluxed for 50 hours. After cooling to room temperature the reaction mixture was diluted with n-hexane and water. The precipitate was collected by filtration and washed repeatedly with tetrahydrofurane and 
water for purification to give $303 \mathrm{mg}(0.76 \mathrm{mmol}, 76 \%)$ of NOP4 as a yellow amorphous solid.

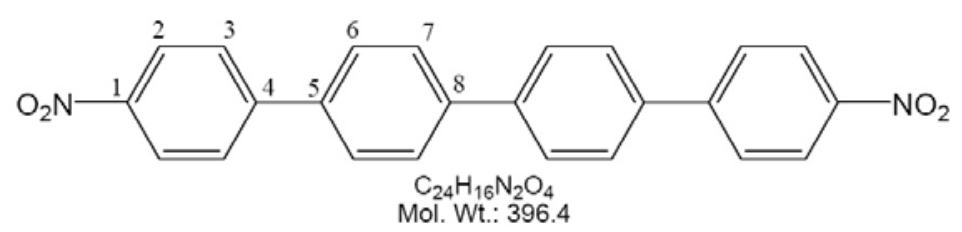

${ }^{1} \mathrm{H}-\mathrm{NMR}\left(\mathrm{CDCl}_{3}:\right.$ trifluoroacetic acid 3:1, $\left.\delta[\mathrm{ppm}]\right): 7.78(4 \mathrm{H}, \mathrm{d}, J=8.4 \mathrm{~Hz}, \mathrm{H}-6), 7.83$ $(4 \mathrm{H}, \mathrm{d}, J=8.4 \mathrm{~Hz}, \mathrm{H}-7), 7.86(4 \mathrm{H}, \mathrm{d}, J=8.8 \mathrm{~Hz}, \mathrm{H}-3), 8.36(4 \mathrm{H}, \mathrm{d}, J=8.8 \mathrm{~Hz}, \mathrm{H}-2)$. ${ }^{13} \mathrm{C}-\mathrm{NMR}\left(\mathrm{CDCl}_{3}\right.$ :trifluoroacetic acid 3:1, $\left.\delta[\mathrm{ppm}]\right): 124.7$ (C-2), 128.2 (C-6, C-7), 128.3 (C-3), 138.3 (C-5), 141.4 (C-8), 146.8 (C-1), 148.6 (C-4).

MS (CI, isobutane): $\mathrm{m} / \mathrm{z} 397.1\left(\mathrm{M}+\mathrm{H}^{+}\right) 100 \%, 398.1\left(\mathrm{M}+\mathrm{H}^{+}\right) 26 \%$.

HR-MS (EI): calcd. for $\mathrm{C}_{24} \mathrm{H}_{16} \mathrm{~N}_{2} \mathrm{O}_{4}$ : 396.1109; found: 366.1110 .

UVvis (dichloromethane): $\lambda_{\max }=347 \mathrm{~nm}$.

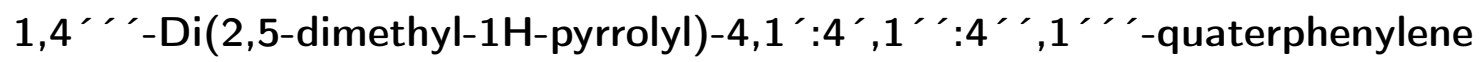 (PYRP4)}

Under an argon atmosphere 4,4'-biphenyldiboronic acid bis(neopentyl glycol)ester (750 mg, $2.00 \mathrm{mmol}$ ), p-(2,5-Dimethyl-1H-pyrrolyl-1-yl)iodbenzene (1.25 g, $4.2 \mathrm{mmol}, 2.1$ equivalents), cesium fluoride (1.22 g, $8.0 \mathrm{mmol}, 4$ equivalents) and tetrakis(triphenylphosphine)-palladium(0) (69 mg, $0.06 \mathrm{mmol}, 3 \mathrm{~mol} \%$ ) were dissolved in $50 \mathrm{~mL}$ of abs. THF and refluxed for 50 hours. The pyrrole protected crude product was washed with tetrahydrofurane and water to give a brown amorphous solid, which can be purified by refluxing for $50 \mathrm{~h}$ with $2.7 \mathrm{~g}$ hydroxylamine hydrochloride (40.0 mmol, 20 equivalents) in ethanol/water/triethylamine (20:11:3). Usually these conditions are applied to achieve cleavage of the pyrrole protecting group. The product was collected by filtration as a light brown solid yielding $886 \mathrm{mg}$ (1.8 mmol, 90\%).

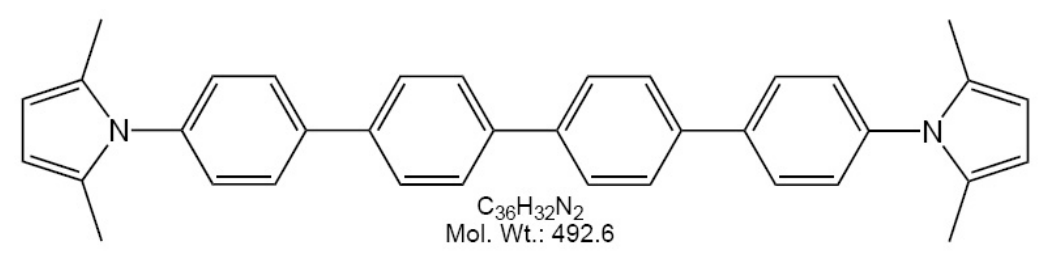

${ }^{1} \mathrm{H}-\mathrm{NMR}\left(\mathrm{CDCl}_{3}, \delta[\mathrm{ppm}]\right): 2.09(12 \mathrm{H}, \mathrm{s}), 5.92(4 \mathrm{H}, \mathrm{s}), 7.30(4 \mathrm{H}, \mathrm{d}, J=8.2 \mathrm{~Hz}), 7.71$ - $7.78(12 \mathrm{H}, \mathrm{m})$.

MS (CI, isobutane): $\mathrm{m} / \mathrm{z} 493.2\left(\mathrm{M}+\mathrm{H}^{+}\right)$.

UVvis (dichloromethane): $\lambda_{\max }=308 \mathrm{~nm}$. 


\section{1,4' '-Diaza-4,1' $: 4^{\prime}, 1^{\prime \prime}: 44^{\prime \prime}, 1{ }^{\prime}{ }^{\prime \prime}$-quaterphenylene (PYP2) [203-205]}

Under argon atmosphere 4,4'-biphenyldiboronic acid bis(neopentyl glycol)ester (361 mg, $1.3 \mathrm{mmol}$ ), 4-iodpyridine (560 mg, $2.7 \mathrm{mmol}, 2.1$ equivalents), cesium fluoride (790 mg, $5.2 \mathrm{mmol}, 4$ equivalents) and tetrakis(triphenylphosphine)-palladium(0) (45 $\mathrm{mg}, 0.039$ mmol, $3 \mathrm{~mol} \%$ ) were dissolved in $60 \mathrm{ml}$ abs. tetrahydrofurane and refluxed for 50 hours. The precipitate was collected by filtration and washed for purification with water, dichloromethane and ethylacetate to give $215 \mathrm{mg}(0.69 \mathrm{mmol}, 54 \%)$ of a colourless amorphous solid. The attempt to crystallize from chloroform and methanol caused only formation of an amorphous chloroform-adduct with even decreased solubility.

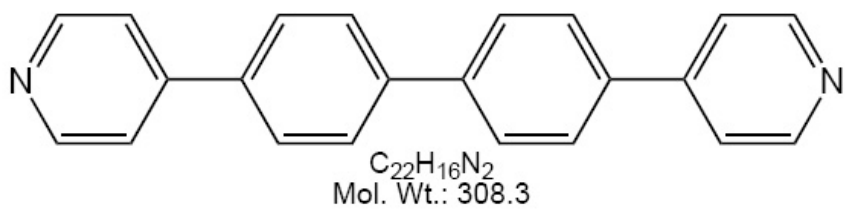

${ }^{1} \mathrm{H}-\mathrm{NMR}$ (acetone- $d_{6}$ :trifluoroacetic acid, $\left.\delta[\mathrm{ppm}]\right): 7.88(4 \mathrm{H}, \mathrm{d}, J=8.3 \mathrm{~Hz}), 8.00(4 \mathrm{H}$, d, $J=8.3 \mathrm{~Hz}), 8.39(4 \mathrm{H}, \mathrm{d}, J=6.6 \mathrm{~Hz}), 8.91(4 \mathrm{H}, \mathrm{d}, J=6.6 \mathrm{~Hz})$.

MS (EI): m/z $308.1\left(\mathrm{M}^{+}\right), 154.0\left(\mathrm{M}^{2}+\right)$.

HR-MS (EI): calcd. for $\mathrm{C}_{16} \mathrm{H}_{16} \mathrm{~N}_{2}$ : 308.1314; found: 308.1316 .

UVvis (dichloromethane): $\lambda_{\max }=302 \mathrm{~nm}$.

\subsubsection{Non-symmetrically functionalised para-Quaterphenylenes}

\subsubsection{Precursors: phenyl building blocks}

p-(2,5-Dimethyl-1H-pyrrolyl-1-yl)iodbenzene (PYRIP) [206, 207] In a $100 \mathrm{~mL}$ flask $1643 \mathrm{mg}$ ( $7.5 \mathrm{mmol})$ p-iodaniline, $0.922 \mathrm{~mL}$ (7.9 mmol, 1.05 equivalents) acetonylacetone and $23 \mathrm{mg} p$-toluene sulfonic acid were solved in toluene and refluxed under Dean-Stark conditions for $5 \mathrm{~h}$. The toluene phase was washed with aqueous sodium hydrogen carbonate and water, dried over sodium sulphate and concentrated. The crude product was purified by chromatography (HE/EE 5:1) to give $1602 \mathrm{mg}(5.4 \mathrm{mmol}, 72 \%)$ of a bluebrown solid.

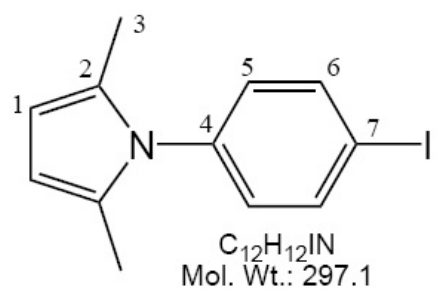

${ }^{1} \mathrm{H}-\mathrm{NMR}\left(\mathrm{CDCl}_{3}, \delta[\mathrm{ppm}]\right): 2.0(6 \mathrm{H}, \mathrm{s}, \mathrm{H}-3), 5.8(2 \mathrm{H}, \mathrm{s}, \mathrm{H}-1), 6.95(2 \mathrm{H}, \mathrm{d}, J=8.5 \mathrm{~Hz}$, 
$\mathrm{H}-5), 7.77$ (2 H, d, $J=8.5 \mathrm{~Hz}, \mathrm{H}-6)$.

${ }^{13} \mathrm{C}-\mathrm{NMR}\left(\mathrm{CDCl}_{3}, \delta[\mathrm{ppm}]\right): 12.9(\mathrm{C}-3), 92.9$ (C-7), 106.1 (C-1), 128.6 (C-2), 130.1 (C-5), 138.3 (C-6), 138.7 (C-4).

MS (CI, isobutane): m/z $297.9\left(\mathrm{M}+\mathrm{H}^{+}\right)$.

4-(4,4,5,5-tetramethyl-1,3,2-dioxaborolyl)- $N, N$-dimethylaniline (NMePEP) [208, 209] A two-neck-flask was charged under argon with $600 \mathrm{mg}$ (3.0 mmol) 4-bromo$N, N$-dimethylaniline, $33 \mathrm{mg}(0.15 \mathrm{mmol}, 5 \mathrm{~mol} \%) \mathrm{Pd}(\mathrm{OAc})_{2}$ and $162 \mathrm{mg}(0.30 \mathrm{mmol}$, 10mol\%) 1-(diphenylphosphino)-2-(2-(diphenylphosphino)phenoxy)benzene. Via syringe were added $20 \mathrm{~mL}$ dry dioxane, $1.7 \mathrm{~mL}$ (12.0 mmol, 4 eqivalents) dry triethylamine and $0.87 \mathrm{~mL}$ (6.0 mmol, 2 equivalents) 4,4,5,5-tetramethyl-1,3,2-dioxaborolane. The reaction mixture was heated to $100^{\circ} \mathrm{C}$ for 20 hours. After cooling to room temperature the reaction mixture was quenched with a sat. solution of $\mathrm{NH}_{4} \mathrm{Cl}$. The aqueous phase was extracted with dichloromethane. The organic phase was dried and concentrated. The crude product was purified by chromatography ( $n$-hexane / ethyl acetate $3: 1+5 \%$ triethylamine) to give $705 \mathrm{mg}(2.85 \mathrm{mmol}, 95 \%)$ of a fawn solid.

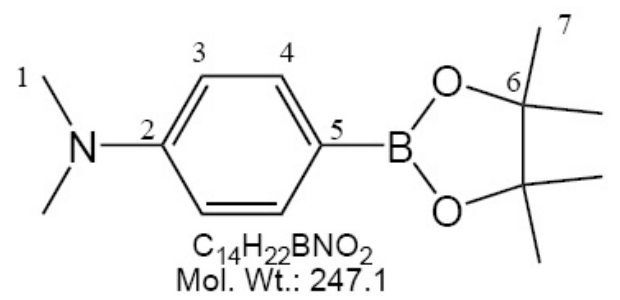

${ }^{1} \mathrm{H}-\mathrm{NMR}\left(\mathrm{CDCl}_{3}, \delta[\mathrm{ppm}]\right): 1.31$ (s, $\left.12 \mathrm{H}, \mathrm{H}-7\right), 2.97$ (s, $\left.6 \mathrm{H}, \mathrm{H}-1\right), 6.68$ (d, $2 \mathrm{H}, J=8.5$ $\mathrm{Hz}, \mathrm{H}-3), 7.68$ (d, $2 \mathrm{H}, J=8.5 \mathrm{~Hz}, \mathrm{H}-4)$.

${ }^{13} \mathrm{C}-\mathrm{NMR}\left(\mathrm{CDCl}_{3}, \delta[\mathrm{ppm}]\right): 24.8$ (C-7), 40.1 (C-1), 83.1 (C-6), 111.2 (C-3), 136.1 (C-4, $\mathrm{C}-5), 152.5$ (C-2).

MS (CI, isobutane): $\mathrm{m} / \mathrm{z} 248.1\left(\mathrm{M}+\mathrm{H}^{+}\right)$.

4-lodpyridine (PYI) [210] 4-Aminopyridine (2 g, $21.3 \mathrm{mmol})$ was dissolved in $20 \mathrm{ml}$ $48 \%$ aqueous $\mathrm{HBF}_{4}$ and cooled to $-10^{\circ} \mathrm{C}$. Powdered $\mathrm{NaNO}_{2}$ (1.6 g. $23.3 \mathrm{mmol}$ ) was slowly added. After 30 minutes most of the solvent was removed with a disposable pipette. A solution of KI (5.6 g, $34.1 \mathrm{mmol})$ in $50 \mathrm{~mL}$ of an acetone:water (40:60) mixture was slowly added. After 10 minutes the reaction mixture was decolourized with aqueous $\mathrm{Na}_{2} \mathrm{~S}_{2} \mathrm{O}_{3}$. The reaction mixture was stirred at room temperature for 8 hours and then neutralised with saturated aqueous sodium carbonate and extracted 3 times with diethyl ether. The organic layer was washed with aqueous $\mathrm{Na}_{2} \mathrm{~S}_{2} \mathrm{O}_{3}$ and water and dried over sodium sulphate. The solvent was carfully removed and the crude product was purified by sublimation to give $2.3 \mathrm{~g}(11.2 \mathrm{mmol}, 53 \%)$ of white crystals.

${ }^{1} \mathrm{H}-\mathrm{NMR}\left(\mathrm{CDCl}_{3}, \delta[\mathrm{ppm}]\right): 7.68(2 \mathrm{H}, \mathrm{d}, J=6.0, \mathrm{H}-2), 8.26(2 \mathrm{H}, \mathrm{d}, J=6.0, \mathrm{H}-1)$. 


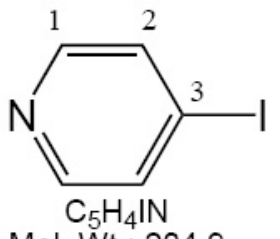

Mol. Wt.: 204.9

${ }^{13} \mathrm{C}-\mathrm{NMR}\left(\mathrm{CDCl}_{3}, \delta[\mathrm{ppm}]\right): 105.4(\mathrm{C}-3), 133.2(\mathrm{C}-2), 150.2(\mathrm{C}-1)$.

MS (CI, isobutane): $\mathrm{m} / \mathrm{z} 205.9\left(\mathrm{M}+\mathrm{H}^{+}\right), 80.0\left(\mathrm{C}_{4} \mathrm{H}_{4} \mathrm{~N}^{+}\right)$.

4-(4,4,5,5-tetramethyl-1,3,2-dioxaborolyl)pyridine (PYPE) [210] To a cooled solution $\left(-78^{\circ} \mathrm{C}\right)$ under argon of 4-iodopyridine $(1 \mathrm{~g}, 4.8 \mathrm{mmol})$ in $60 \mathrm{~mL}$ dry diethyl ether was slowly added a $1.6 \mathrm{M} n$-buthyl-lithium solution $(3.8 \mathrm{~mL}, 6.1 \mathrm{mmol}, 1.2$ equivalents) followed 30 minutes later by trimethyl borate $(0.7 \mathrm{~mL}, 6.1 \mathrm{mmol}, 1.2$ equivalents $)$. The reaction mixture was allowed to warm up to room temperature and stirred for 3 hours. Then pinacol (775 mg, $6.6 \mathrm{mmol}, 1.3$ equivalents) was added and 10 minutes later glacial acetic acid $(0.3 \mathrm{~mL}, 5 \mathrm{mmol})$. The reaction mixture was stirred for 8 hours, filtered through Celite, the filter washed with dichloromethane : ethanol (3:1) and the filtrate was evaporated in vacuo. The crude product was washed with acetone to give $636 \mathrm{mg}$ (3.1 mmol, 65\%) of an amorphous white solid.

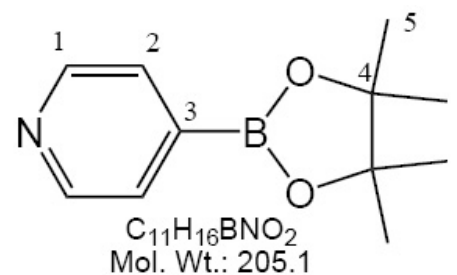

${ }^{1} \mathrm{H}-\mathrm{NMR}\left(\mathrm{CDCl}_{3}, \delta[\mathrm{ppm}]\right): 1.34$ (s, $\left.12 \mathrm{H}, \mathrm{H}-5\right), 7.61$ (d, $\left.2 \mathrm{H}, J=5.5 \mathrm{~Hz}, \mathrm{H}-2\right), 8.63$ (d, $2 \mathrm{H}, J=5.5 \mathrm{~Hz}, \mathrm{H}-1)$.

${ }^{13} \mathrm{C}-\mathrm{NMR}\left(\mathrm{CDCl}_{3}, \delta[\mathrm{ppm}]\right): 24.8(\mathrm{C}-5), 84.5(\mathrm{C}-4), 128.7$ (C-2, C-3), $149.0(\mathrm{C}-1)$. MS (CI, isobutane): $\mathrm{m} / \mathrm{z} 441.1\left(2 \mathrm{M}+\mathrm{H}^{+}\right), 206.0\left(\mathrm{M}+\mathrm{H}^{+}\right)$.

\subsubsection{Precursors: biphenyl building blocks}

General procedure A for the synthesis 4'-functionalised 2,5-dimethyl-1H-pyrrole protected 4-amino-biphenyles A 2-neck-flask equipped with condenser is charged with $600 \mathrm{mg}(2.0 \mathrm{mmol})$ of 4- (2,5-Dimethyl-1H-pyrrol-1-yl)iodobenzene (PYRIP), $2.1 \mathrm{mmol}$ (1.05 equivalents) of a 4-substituted phenyl boronic acid or ester, $912 \mathrm{mg}$ (6.0 mmol, 3 equivalents) of cesium fluoride, and $69 \mathrm{mg}(0.06 \mathrm{mmol}, 3 \mathrm{~mol} \%)$ of $\left[\mathrm{Pd}\left(\mathrm{PPh}_{3}\right)_{4}\right]$ are dissolved in $40 \mathrm{~mL}$ abs. THF under an argon atmosphere and refluxed for 15 hours. After cooling to room temperature the solution is diluted with $50 \mathrm{~mL}$ of dichloromethane, washed with water, dried over sodium sulphate, and concentrated in vacuo. The resulting 
residue is purified by column chromatography on silica to obtain the desired functionalised biphenyl.

4-(2,5-Dimethyl-1H-pyrrole)-4'-methoxy-biphenyl (MOPYRP2) Treatment of (4methoxy phenyl)-boronic acid with PYRIP according to the general Procedure A gave 549 mg (1.98 mmol, 99\%) of MOPYRP2 after column chromatography using $n$-hexane/ethyl acetate $3: 1$ as eluent.

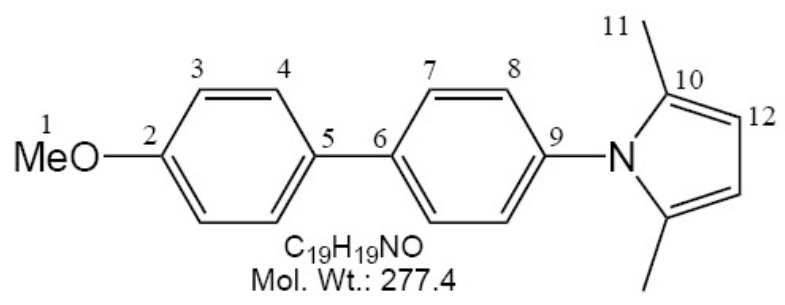

${ }^{1} \mathrm{H}-\mathrm{NMR}\left(\mathrm{CDCl}_{3}, \delta[\mathrm{ppm}]\right): 2.07(6 \mathrm{H}, \mathrm{s}, \mathrm{H}-11), 3.86(3 \mathrm{H}, \mathrm{s}, \mathrm{H}-1), 5.92(2 \mathrm{H}, \mathrm{s}, \mathrm{H}-12)$, $7.00(2 \mathrm{H}, \mathrm{d}, J=8.7 \mathrm{~Hz}, \mathrm{H}-3), 7.24(2 \mathrm{H}, \mathrm{d}, J=8.2 \mathrm{~Hz}, \mathrm{H}-8), 7.57(2 \mathrm{H}, \mathrm{d}, J=8.7 \mathrm{~Hz}$, $\mathrm{H}-4), 7.62(2 \mathrm{H}, \mathrm{d}, J=8.2 \mathrm{~Hz}, \mathrm{H}-7)$.

${ }^{13} \mathrm{C}-\mathrm{NMR}\left(\mathrm{CDCl}_{3}, \delta[\mathrm{ppm}]\right): 13.1$ (C-11), 55.3 (C-1), 105.7 (C-12), 114.3 (C-3), 127.1 (C-7), 128.1 (C-4), 128.4 (C-8), 128.8 (C-10), 132.7 (C-5), 137.5 (C-9), 140.1 (C-6), 159.4 (C-2).

MS (CI, isobutane): $\mathrm{m} / \mathrm{z} 278.2\left(\mathrm{M}+\mathrm{H}^{+}\right)$.

HR-MS (EI): calcd. for $\mathrm{C}_{19} \mathrm{H}_{19} \mathrm{NO}$ : 277.1467; found: 277.1467.

4-(2,5-Dimethyl-1H-pyrrole)-4'-chloro-biphenyl (CLPYRP2) Treatment of (4-chloro phenyl)-boronic acid with PYRIP according to the general Procedure A gave 535 mg (1.90 mmol, 95\%) of CLPYRP2 after column chromatography using $n$-hexane/ethyl acetate $3: 1$ as eluent.

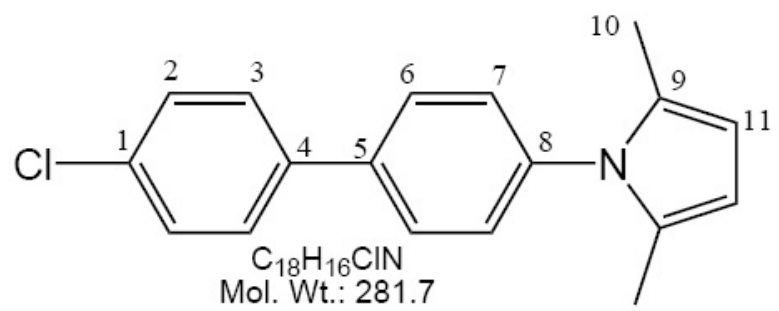

${ }^{1} \mathrm{H}-\mathrm{NMR}\left(\mathrm{CDCl}_{3}, \delta[\mathrm{ppm}]\right): 2.12(6 \mathrm{H}, \mathrm{s}, \mathrm{H}-10), 5.98(2 \mathrm{H}, \mathrm{s}, \mathrm{H}-11), 7.32(2 \mathrm{H}, \mathrm{d}, J=$ $8.3 \mathrm{~Hz}, \mathrm{H}-7), 7.47(2 \mathrm{H}, \mathrm{d}, J=8.5 \mathrm{~Hz}, \mathrm{H}-2), 7.59(2 \mathrm{H}, \mathrm{d}, J=8.5 \mathrm{~Hz}, \mathrm{H}-3), 7.67$ (2 H, $\mathrm{d}, J=8.3 \mathrm{~Hz}, \mathrm{H}-6)$.

${ }^{13} \mathrm{C}-\mathrm{NMR}\left(\mathrm{CDCl}_{3}, \delta[\mathrm{ppm}]\right): 13.0$ (C-10), 105.9 (C-11), 127.5 (C-6), 128.3 (C-3), 128.5 
(C-7), 128.7 (C-9), 128.9 (C-2), 133.7 (C-1), 138.4 (C-8*), 138.6 (C-4*), 139.1 (C-5). MS (CI, isobutane): $\mathrm{m} / \mathrm{z} 282.2\left(\mathrm{M}+\mathrm{H}^{+}\right)$.

HR-MS (EI): calcd. for $\mathrm{C}_{18} \mathrm{H}_{16} \mathrm{ClN}$ : 281.0971; found: 281.0971 .

4-(2,5-Dimethyl-1H-pyrrole)-4'-cyano-biphenyl (CNPYRP2) Treatment of (4-cyano phenyl)-boronic acid with PYRIP according to the general Procedure A gave $502 \mathrm{mg}$ (1.84 mmol, 92\%) of CNPYRP2 after column chromatography using $n$-hexane/ethyl acetate 3:1 as eluent.

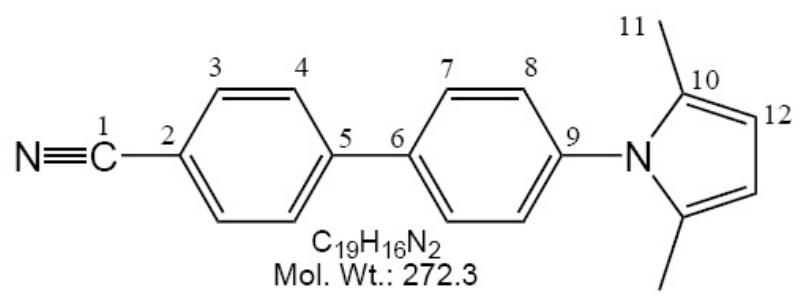

${ }^{1} \mathrm{H}-\mathrm{NMR}\left(\mathrm{CDCl}_{3}, \delta[\mathrm{ppm}]\right): 2.10(6 \mathrm{H}, \mathrm{s}, \mathrm{H}-11), 5.95(2 \mathrm{H}, \mathrm{s}, \mathrm{H}-12), 7.34(2 \mathrm{H}, \mathrm{d}, J=$ $8.3 \mathrm{~Hz}, \mathrm{H}-8), 7.70(2 \mathrm{H}, \mathrm{d}, J=8.3 \mathrm{~Hz}, \mathrm{H}-7), 7.75$ (2 H, d, $J=8.5 \mathrm{~Hz}, \mathrm{H}-4), 7.77$ (2 H, $\mathrm{d}, J=8.3 \mathrm{~Hz}, \mathrm{H}-3)$.

${ }^{13} \mathrm{C}-\mathrm{NMR}\left(\mathrm{CDCl}_{3}, \delta[\mathrm{ppm}]\right): 13.0$ (C-11), 105.9 (C-12), 111.2 (C-2), 118.6 (C-1), 127.6 $\left(\mathrm{C}-4^{*}\right), 127.8$ (C-7*), 128.6 (C-10), 128.8 (C-8), 132.6 (C-3), 138.2 (C-9), 139.3 (C-6), $144.5(\mathrm{C}-5)$.

MS (EI): m/z $272.1\left(\mathrm{M}^{+}\right)$.

HR-MS (EI): calcd. for $\mathrm{C}_{19} \mathrm{H}_{16} \mathrm{~N}_{2}$ : 272.1313; found: 272.1314 .

4-(2,5-Dimethyl-1H-pyrrole)-4'-N,N-dimethylamino-biphenyl (NMePYRP2) Treatment of 4-4,4,5,5-tetramethyl-1,3,2-dioxaborolyl)-N,N-dimethylaniline (NMePEP) with PYRIP according to the general Procedure A gave $540 \mathrm{mg}$ (1.86 mmol, 93\%) of NMePYRP2 after column chromatography using $n$-hexane/ethyl acetate $3: 1+0.5 \%$ triethylamine as eluent.

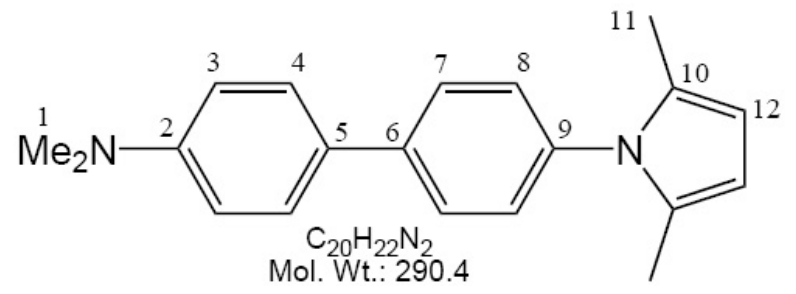

${ }^{1} \mathrm{H}-\mathrm{NMR}\left(\mathrm{CDCl}_{3}, \delta[\mathrm{ppm}]\right): 2.09(6 \mathrm{H}, \mathrm{s}, \mathrm{H}-11), 3.02(6 \mathrm{H}, \mathrm{s}, \mathrm{H}-1), 5.93(2 \mathrm{H}, \mathrm{s}, \mathrm{H}-12)$, $6.83(2 \mathrm{H}, \mathrm{d}, J=8.7 \mathrm{~Hz}, \mathrm{H}-3), 7.23(2 \mathrm{H}, \mathrm{d}, J=8.3 \mathrm{~Hz}, \mathrm{H}-8), 7.56(2 \mathrm{H}, \mathrm{d}, J=8.7 \mathrm{~Hz}$, 
H-4), $7.63(2 \mathrm{H}, \mathrm{d}, J=8.3 \mathrm{~Hz}, \mathrm{H}-7)$.

${ }^{13} \mathrm{C}-\mathrm{NMR}\left(\mathrm{CDCl}_{3}, \delta[\mathrm{ppm}]\right): 13.1$ (C-11), 40.5 (C-1), 105.5 (C-12), 112.7 (C-3), 126.6 (C-7), 127.6 (C-4), 128.0 (C-5), 128.3 (C-8), 128.9 (C-10), 136.8 (C-9), 140.5 (C-6), 150.1 (C-2).

MS (CI, isobutane): $\mathrm{m} / \mathrm{z} 291.1\left(\mathrm{M}+\mathrm{H}^{+}\right)$.

HR-MS (EI): calcd. for $\mathrm{C}_{20} \mathrm{H}_{22} \mathrm{~N}_{2}$ : 290.1783; found: 290.1788 .

4-(2,5-Dimethyl-1H-pyrrole)-4'-nitro-biphenyl (NOPYRP2) Treatment of (4-nitro phenyl)-boronic acid with PYRIP according to the general Procedure A gave $473 \mathrm{mg}$ (1.62 mmol, $81 \%$ ) of NOPYRP2 after column chromatography using $n$-hexane/ethyl acetate 3:1 as eluent.

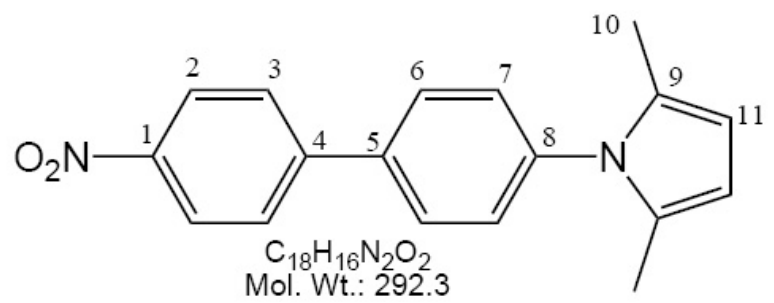

${ }^{1} \mathrm{H}-\mathrm{NMR}\left(\mathrm{CDCl}_{3}, \delta[\mathrm{ppm}]\right): 2.09(6 \mathrm{H}, \mathrm{s}, \mathrm{H}-10), 5.94(2 \mathrm{H}, \mathrm{s}, \mathrm{H}-11), 7.35(2 \mathrm{H}, \mathrm{d}, J=$ $8.3 \mathrm{~Hz}, \mathrm{H}-7), 7.73(2 \mathrm{H}, \mathrm{d}, J=8.3 \mathrm{~Hz}, \mathrm{H}-6), 7.79(2 \mathrm{H}, \mathrm{d}, J=8.8 \mathrm{~Hz}, \mathrm{H}-3), 8.33(2 \mathrm{H}$, $\mathrm{d}, J=8.8 \mathrm{~Hz}, \mathrm{H}-2)$.

${ }^{13} \mathrm{C}-\mathrm{NMR}\left(\mathrm{CDCl}_{3}, \delta[\mathrm{ppm}]\right): 13.0(\mathrm{C}-10), 106.2$ (C-11), 124.2 (C-2), 127.8 (C-3), 128.0 (C-6), 128.7 (C-9), 128.8 (C-7), 137.9 (C-5), 139.7 (C-8), 146.5 (C-4), 147.3 (C-1).

MS (CI, isobutane): $\mathrm{m} / \mathrm{z} 293.3\left(\mathrm{M}+\mathrm{H}^{+}\right)$.

HR-MS (EI): calcd. for $\mathrm{C}_{18} \mathrm{H}_{16} \mathrm{~N}_{2} \mathrm{O}_{2}$ : 292.1212; found: 292.1211.

4-(2,5-Dimethyl-1H-pyrrole)-4 '-bromo-biphenyl (BRPYRP2) Treatment of (4-bromo phenyl)-boronic acid with PYRIP according to the general Procedure A gave 639 mg (1.96 mmol, 98\%) of BRPYRP2 after column chromatography using $n$-hexane/ethyl acetate $3: 1$ as eluent.

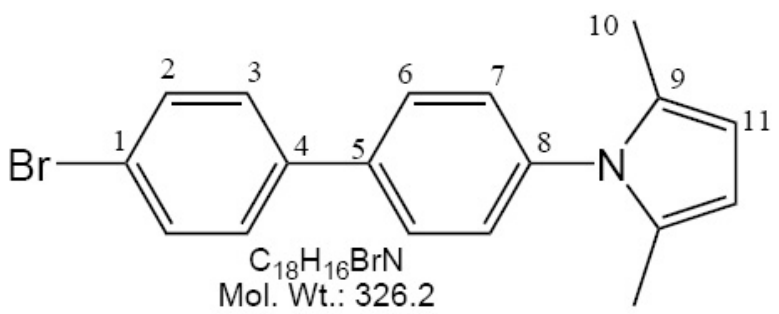

${ }^{1} \mathrm{H}-\mathrm{NMR}\left(\mathrm{CDCl}_{3}, \delta[\mathrm{ppm}]\right): 2.07(6 \mathrm{H}, \mathrm{s}, \mathrm{H}-10), 5.92(2 \mathrm{H}, \mathrm{s}, \mathrm{H}-11), 7.27(2 \mathrm{H}, \mathrm{d}, J=$ 
$8.3 \mathrm{~Hz}, \mathrm{H}-7), 7.49(2 \mathrm{H}, \mathrm{d}, J=8.5 \mathrm{~Hz}, \mathrm{H}-3), 7.59(2 \mathrm{H}, \mathrm{d}, J=8.3 \mathrm{~Hz}, \mathrm{H}-2), 7.63(2 \mathrm{H}$, $\mathrm{d}, J=8.3 \mathrm{~Hz}, \mathrm{H}-6)$.

${ }^{13} \mathrm{C}-\mathrm{NMR}\left(\mathrm{CDCl}_{3}, \delta[\mathrm{ppm}]\right): 13.0$ (C-10), 105.9 (C-11), 121.9 (C-1), 127.5 (C-6), 128.6 $\left(\mathrm{C}-7^{*}\right), 128.7\left(\mathrm{C}-3^{*}\right), 128.8$ (C-9), 131.9 (C-2), 138.5 (C-8), 139.0 (C-4), 139.2 (C-5).

MS (CI, isobutane): $\mathrm{m} / \mathrm{z} 326.1\left(\mathrm{M}+\mathrm{H}^{+}\right), 328.1\left(\mathrm{M}+\mathrm{H}^{+}\right)$.

HR-MS (EI): calcd. for $\mathrm{C}_{18} \mathrm{H}_{16} \mathrm{BrN}$ : 325.0466; found: 325.0467 .

General procedure B for the synthesis 4'-functionalised 4-iodo-biphenyles from the corresponding protected 4-amino-biphenyls 1.9 mmol of a $4^{\prime}$-substituted 4-(2,5dimethyl-1H-pyrrol-1-yl)biphenyl are dissolved in a mixture of $20 \mathrm{~mL}$ of $2 \mathrm{~N}$ sulphuric acid and $20 \mathrm{~mL}$ of acetonitrile and cooled to $-5{ }^{\circ} \mathrm{C}$. At this temperature $400 \mathrm{mg}(5.9 \mathrm{mmol}, 3$ equivalents) of sodium nitrite dissolved in $5 \mathrm{~mL}$ of water are slowly added dropwise. After stirring for half an hour $1.30 \mathrm{~g}$ (7.6 mmol, 4 equivalents) of potassium iodide dissolved in 5 $\mathrm{mL}$ of water are added dropwise at $-5{ }^{\circ} \mathrm{C}$. After removing of the cooling bath, the reaction mixture is stirred for $1 \mathrm{~h}$, heated up to $60{ }^{\circ} \mathrm{C}$ for a short period of time, and then stirred at room temperature for $5 \mathrm{~h}$. The reaction mixture is neutralised with sat. aq. $\mathrm{Na}_{2} \mathrm{SO}_{4}$. The aqueous layer is repeatedly extracted with dichloromethane. The combined organic layers are washed with water, an aq. solution of sodium thiosulphate, and again water, dried over sodium sulphate, and concentrated in vacuo. The crude product is spread on silica gel purified by flash chromatography on silica using $n$-hexane/ethyl acetate 3:1 as eluent.

4-lodo-4'-methoxy-biphenyl (MOIP2) [112, 211-214] Treatment of MOPYRP2 according to general procedure B gave $324 \mathrm{mg}$ (1.05 mmol, 55\%) of MOIP2.

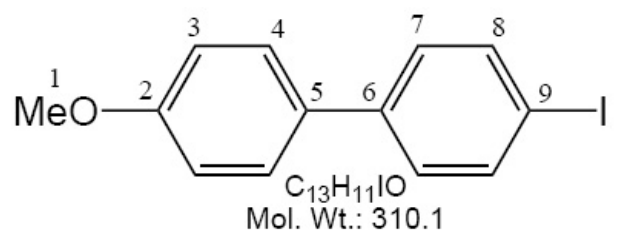

${ }^{1} \mathrm{H}-\mathrm{NMR}\left(\mathrm{CDCl}_{3}, \delta[\mathrm{ppm}]\right): 3.84(3 \mathrm{H}, \mathrm{s}, \mathrm{H}-1), 6.96(2 \mathrm{H}, \mathrm{d}, J=8.7 \mathrm{~Hz}, \mathrm{H}-3), 7.27(2 \mathrm{H}$, d, $J=8.3 \mathrm{~Hz}, \mathrm{H}-7), 7.47(2 \mathrm{H}, \mathrm{d}, J=8.7 \mathrm{~Hz}, \mathrm{H}-4), 7.72(2 \mathrm{H}, \mathrm{d}, J=8.3 \mathrm{~Hz}, \mathrm{H}-8)$.

${ }^{13} \mathrm{C}-\mathrm{NMR}\left(\mathrm{CDCl}_{3}, \delta[\mathrm{ppm}]\right): 55.3$ (C-1), 92.1 (C-9), 114.3 (C-3), 127.9 (C-4), 128.6 (C-7), 132.5 (C-5), 137.7 (C-8), 140.3 (C-6), 159.4 (C-2).

MS (EI): m/z $310.0\left(\mathrm{M}^{+}\right), 184.1\left({\left.\mathrm{M}-\mathrm{I}^{+}\right) .}^{\circ}\right.$

HR-MS (EI): calcd. for $\mathrm{C}_{13} \mathrm{H}_{11} \mathrm{IO}: 309.9854$; found: 309.9853.

4-Chloro-4'-iodo-biphenyl (CLIP2) [211] Treatment of CLPYRP2 according to general procedure B gave $191 \mathrm{mg}(0.61 \mathrm{mmol}, 32 \%)$ of CLIP2. 
The analytical data were in agreement with those published by R.N. Young.

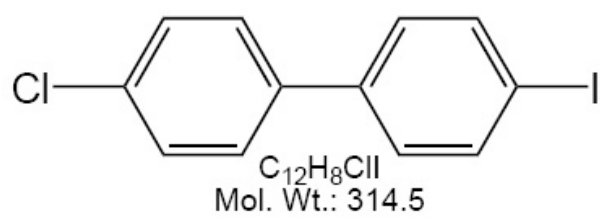

4-Cyano-4'-iodo-biphenyl (CNIP2) [215, 216] Treatment of CNPYRP2 according to general procedure B gave $336 \mathrm{mg}(1.10 \mathrm{mmol}, 58 \%)$ of CNIP2.

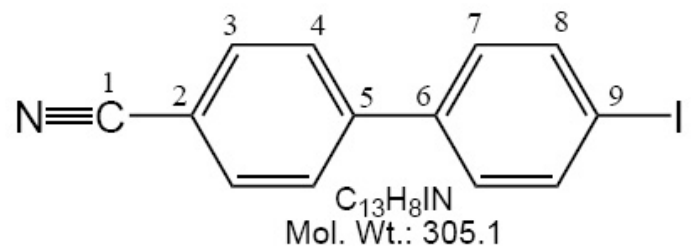

${ }^{1} \mathrm{H}-\mathrm{NMR}\left(\mathrm{CDCl}_{3}, \delta[\mathrm{ppm}]\right): 7.31(2 \mathrm{H}, \mathrm{d}, J=8.4 \mathrm{~Hz}, \mathrm{H}-7), 7.63(2 \mathrm{H}, \mathrm{d}, J=8.3 \mathrm{~Hz}$, $\mathrm{H}-4), 7.72(2 \mathrm{H}, \mathrm{d}, J=8.3 \mathrm{~Hz}, \mathrm{H}-3), 7.80(2 \mathrm{H}, \mathrm{d}, J=8.4 \mathrm{~Hz}, \mathrm{H}-8)$.

${ }^{13} \mathrm{C}-\mathrm{NMR}\left(\mathrm{CDCl}_{3}, \delta[\mathrm{ppm}]\right): 94.8(\mathrm{C}-9), 111.3(\mathrm{C}-2), 118.6(\mathrm{C}-1), 127.4(\mathrm{C}-4), 128.9$ (C7), 132.6 (C-3), 138.2 (C-8), 138.5 (C-6), 144.4 (C-5).

MS (CI, isobutane): $\mathrm{m} / \mathrm{z} 306.2\left(\mathrm{M}+\mathrm{H}^{+}\right), 280.2\left([\mathrm{M}-\mathrm{I}+2 \mathrm{H}]^{+}\right)$.

HR-MS (CI, isobutane): calcd. for $\mathrm{C}_{13} \mathrm{H}_{9} \mathrm{IN}$ : 305.9779; found: 305.9779 .

4-N,N-Dimethylamino-4 '-iodo-biphenyl (NMeIP2) [217, 218] Treatment of NMePYRP2 according to general procedure B but using $3 \mathrm{~N}$ sulphuric acid instead of 2 $\mathrm{N}$ and $n$-hexane / ethyl acetate $3: 1+0.5 \%$ triethylamine as the eluent for the flashchromatography gave $443 \mathrm{mg}(1.37 \mathrm{mmol}, 68 \%)$ of NMeIP2.

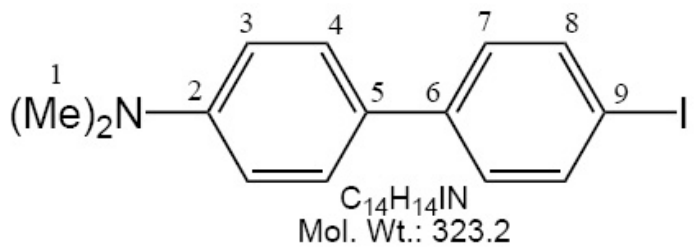

${ }^{1} \mathrm{H}-\mathrm{NMR}\left(\mathrm{CDCl}_{3}, \delta[\mathrm{ppm}]\right): 2.99(6 \mathrm{H}, \mathrm{s}, \mathrm{H}-1), 6.78(2 \mathrm{H}, \mathrm{d}, J=8.7 \mathrm{~Hz}, \mathrm{H}-3), 7.29(2 \mathrm{H}$, $\mathrm{d}, J=8.3 \mathrm{~Hz}, \mathrm{H}-7), 7.45(2 \mathrm{H}, \mathrm{d}, J=8.7 \mathrm{~Hz}, \mathrm{H}-4), 7.69(2 \mathrm{H}, \mathrm{d}, J=8.3 \mathrm{~Hz}, \mathrm{H}-8)$.

${ }^{13} \mathrm{C}-\mathrm{NMR}\left(\mathrm{CDCl}_{3}, \delta[\mathrm{ppm}]\right): 40.4(\mathrm{C}-1), 91.1$ (C-9), 112.7 (C-3), 125.9 (C-5), $127.4(\mathrm{C}-4)$, 128.1 (C-7), 137.6 (C-8), 140.7 (C-6), 150.2 (C-2).

MS (CI, isobutane): $\mathrm{m} / \mathrm{z} 323.9\left(\mathrm{M}+\mathrm{H}^{+}\right), 198.1\left([\mathrm{M}-\mathrm{I}+2 \mathrm{H}]^{+}\right)$.

HR-MS (EI): calcd. for $\mathrm{C}_{14} \mathrm{H}_{14} \mathrm{NI}$ : 323.0171; found: 323.0170 . 
4-lodo-4'-nitro-biphenyl (NOIP2) [112, 214, 219, 220] Treatment of NOPYRP2 according to general procedure B gave $167 \mathrm{mg}$ (0.51 mmol, 27\%) of NOIP2.

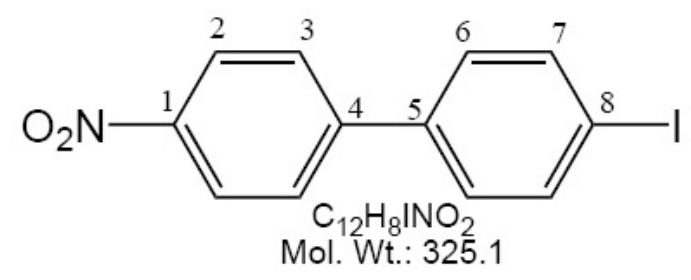

${ }^{1} \mathrm{H}-\mathrm{NMR}\left(\mathrm{CDCl}_{3}, \delta[\mathrm{ppm}]\right): 7.35(2 \mathrm{H}, \mathrm{d}, J=8.3 \mathrm{~Hz}, \mathrm{H}-6), 7.70(2 \mathrm{H}, \mathrm{d}, J=8.8 \mathrm{~Hz}$, $\mathrm{H}-3), 7.83(2 \mathrm{H}, d, \mathrm{~J}=8.3 \mathrm{~Hz}, \mathrm{H}-7), 8.29(2 \mathrm{H}, d, \mathrm{~J}=8.8 \mathrm{~Hz}, \mathrm{H}-2)$.

${ }^{13} \mathrm{C}-\mathrm{NMR}\left(\mathrm{CDCl}_{3}, \delta[\mathrm{ppm}]\right): 95.1$ (C-8), 124.2 (C-2), 127.6 (C-3), 129.0 (C-6), 138.2 (C5), 138.3 (C-7), 146.4 (C-4), 147.3 (C-1).

MS (CI, isobutane): $\mathrm{m} / \mathrm{z} 326.1\left(\mathrm{M}+\mathrm{H}^{+}\right), 200.1\left([\mathrm{M}-\mathrm{I}+2 \mathrm{H}]^{+}\right)$.

HR-MS (CI, isobutane): calcd. for $\mathrm{C}_{12} \mathrm{H}_{9} \mathrm{NO}_{2} \mathrm{I}$ : 325.9678; found: 325.9678 .

\subsubsection{Precursors: terphenylene building blocks}

1-Bromo-4 ' '-methoxy-4,1':4',1' '-terphenylene (MOBRP3) Under an argon atmosphere $700 \mathrm{mg}(2.25 \mathrm{mmol})$ of MOIP2, $452 \mathrm{mg}$ (2.4 mmol, 1.1 equivalents) of 4-bromophenylboronic acid, $1026 \mathrm{mg}$ (6.75 mmol, 3.0 equivalents) of cesium fluoride, and $78 \mathrm{mg}$ $(0.067 \mathrm{mmol}, 3 \mathrm{~mol} \%)$ of $\left[\mathrm{Pd}\left(\mathrm{PPh}_{3}\right)_{4}\right]$ were dissolved in $50 \mathrm{~mL}$ of abs. THF and refluxed for 30 hours. After cooling to room temperature the reaction mixture was diluted with $n$-hexane and water and the precipitate was collected by filtration. The crude product was washed with THF, ethyl acetate, and water to give $641 \mathrm{mg}$ (1.89 mmol, 84\%) of MOBRP3 as a fawn amorphous solid.

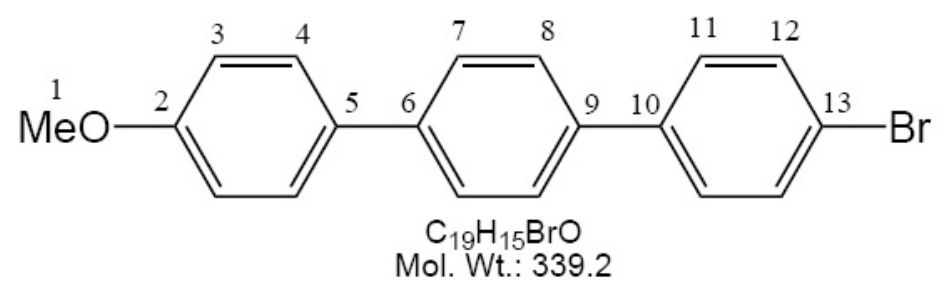

${ }^{1} \mathrm{H}-\mathrm{NMR}\left(\mathrm{CDCl}_{3}, \delta[\mathrm{ppm}]\right): 3.85(3 \mathrm{H}, \mathrm{s}, \mathrm{H}-1), 6.99(2 \mathrm{H}, \mathrm{d}, J=8.4 \mathrm{~Hz}, \mathrm{H}-3), 7.48(2$ $\mathrm{H}, \mathrm{d}, J=8.2 \mathrm{~Hz}, \mathrm{H}-11), 7.56(4 \mathrm{H}, \mathrm{d}, J=8.2 \mathrm{~Hz}, \mathrm{H}-4, \mathrm{H}-12), 7.59(2 \mathrm{H}, \mathrm{d}, J=8.2 \mathrm{~Hz}$, $\left.\mathrm{H}-7^{*}\right), 7.62\left(2 \mathrm{H}, \mathrm{d}, J=8.2 \mathrm{~Hz}, \mathrm{H}-8^{*}\right)$.

${ }^{13} \mathrm{C}-\mathrm{NMR}\left(\mathrm{CDCl}_{3}, \delta[\mathrm{ppm}]\right): 55.3(\mathrm{C}-1), 114.3$ (C-3), $121.4(\mathrm{C}-13), 127.1\left(\mathrm{C}-7^{*}\right), 127.2$ $\left(\mathrm{C}-8^{*}\right), 128.0$ (C-4), 128.4 (C-11), 131.9 (C-12), 133.0 (C-5), 138.2 (C-9), 139.1 (C-6*), $140.1\left(\mathrm{C}-10^{*}\right), 159.3(\mathrm{C}-2)$. 
MS (CI, isobutane): $\mathrm{m} / \mathrm{z} 339.1\left(\mathrm{M}+\mathrm{H}^{+}\right), 340.1\left(\mathrm{M}+\mathrm{H}^{+}\right)$.

HR-MS (EI): calcd. for $\mathrm{C}_{19} \mathrm{H}_{15} \mathrm{BrO}$ : 338.0306; found: 338.0305 .

1-(4,4,5,5-tetramethyl-1,3,2-dioxaborolyl)-4' '-methoxy-4,1' $: 4^{\prime}, 1^{\prime \prime}$ '-terphenylene (MOPEP3)

A two-neck-flask was charged with $244 \mathrm{mg}(0.72 \mathrm{mmol})$ of MOBRP3, $8 \mathrm{mg}$ (0.036 mmol, $5 \mathrm{~mol} \%)$ of $\mathrm{Pd}(\mathrm{OAc})_{2}$, and $39 \mathrm{mg}(0.072 \mathrm{mmol}, 10 \mathrm{~mol} \%)$ of 1-(diphenylphosphino)-2-(2(diphenylphosphino)phenoxy)benzene under an argon atmosphere. $20 \mathrm{~mL}$ of dry 1,4dioxane, $0.4 \mathrm{~mL}$ (2.9 mmol, 4 eqivalents) of dry triethylamine, and $0.21 \mathrm{~mL}(1.44 \mathrm{mmol}$, 2 equivalents) of 4,4,5,5-tetramethyl-1,3,2-dioxaborolane were added via syringe. The reaction mixture was heated to $100^{\circ} \mathrm{C}$ for 30 hours. After cooling to room temperature the reaction mixture was quenched with a sat. aqu. $\mathrm{NH}_{4} \mathrm{Cl}$. The aqueous phase was extracted repeatedly with dichloromethane. The organic phases were dried with sodium sulphate and concentrated in vacuo. The crude product was purified by column chromatography on silica using $n$-hexane/ ethyl acetate $1: 1+5 \%$ triethylamine as eluent to give $188 \mathrm{mg}$ (0.49 mmol, $68 \%)$ of MOPEP3 as a fawn solid.

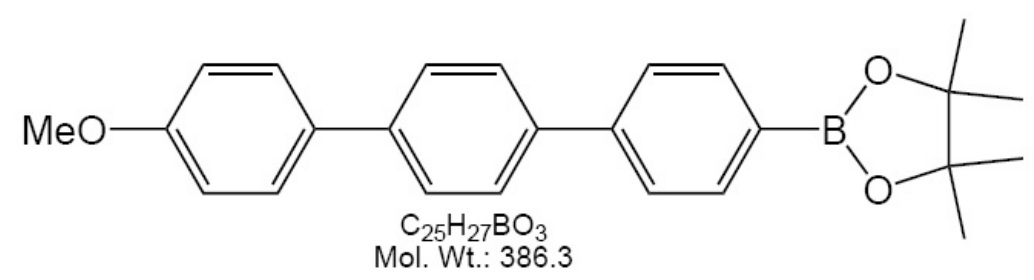

${ }^{1} \mathrm{H}-\mathrm{NMR}\left(\mathrm{CDCl}_{3}, \delta[\mathrm{ppm}]\right): 1.38(\mathrm{~s}, 12 \mathrm{H}), 3.86(\mathrm{~s}, 3 \mathrm{H}), 7.00(\mathrm{~d}, 2 \mathrm{H}, J=8.3 \mathrm{~Hz}), 7.59$ $(\mathrm{d}, 2 \mathrm{H}, J=8.3 \mathrm{~Hz}), 7.63-7.70(\mathrm{~m}, 6 \mathrm{H}), 7.92(\mathrm{~d}, 2 \mathrm{H}, J=7.8 \mathrm{~Hz})$.

${ }^{13} \mathrm{C}-\mathrm{NMR}\left(\mathrm{CDCl}_{3}, \delta[\mathrm{ppm}]\right): 24.8,55.3,83.7,114.2,127.0,127.5,128.0$ (2 C), 133.1, 134.7, 135.2, 140.0, 140.7, 143.3, 159.2.

MS (CI, isobutane): $\mathrm{m} / \mathrm{z} 387.5\left(\mathrm{M}+\mathrm{H}^{+}\right)$.

HR-MS (EI): calcd. for $\mathrm{C}_{25} \mathrm{H}_{27} \mathrm{BO}_{3}$ : 386.2053; found: 386.2052 .

1-Cyano-4' '-bromo-4,1':4',1' '-terphenylene (CNBRP3) [221] Under an argon atmosphere $470 \mathrm{mg}$ (1.5 mmol) of CNIP2, $452 \mathrm{mg}$ (1.6 mmol, 1.1 equivalents) of 4 bromophenylboronic acid, $684 \mathrm{mg}$ ( $4.5 \mathrm{mmol}, 3.0$ equivalents) of cesium fluoride, and $52 \mathrm{mg}(0.045 \mathrm{mmol}, 3 \mathrm{~mol} \%)$ of $\left[\mathrm{Pd}\left(\mathrm{PPh}_{3}\right)_{4}\right]$ were dissolved in $50 \mathrm{~mL}$ of abs. THF and refluxed for 30 hours. After cooling to room temperature the reaction mixture was diluted with n-hexane and water and the precipitate was collected by filtration. The crude product was washed with THF, ethyl acetate, and water to give $255 \mathrm{mg}$ (0.76 mmol, 51\%) of CNBRP3 as a white amorphous solid.

${ }^{1} \mathrm{H}-\mathrm{NMR}\left(\mathrm{CDCl}_{3}, \delta[\mathrm{ppm}]\right): 7.49(2 \mathrm{H}, \mathrm{d}, J=8.5 \mathrm{~Hz}, \mathrm{H}-11), 7.58(2 \mathrm{H}, \mathrm{d}, J=8.5 \mathrm{~Hz}$, 


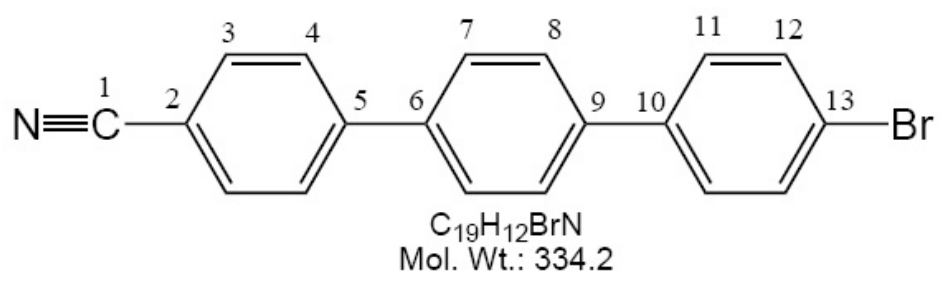

H-12), 7.66 (4 H, s, H-7, H-8), 7.71 (2 H, d, J=8.6 Hz, H-4), 7.73 (2 H, d, J = not resolved, H-3).

${ }^{13} \mathrm{C}-\mathrm{NMR}\left(\mathrm{CDCl}_{3}, \delta[\mathrm{ppm}]\right): 111.1(\mathrm{C}-2), 118.8$ (C-1), $122.0(\mathrm{C}-13), 127.5\left(\mathrm{C}-7^{*}\right), 127.6$ (C-8*), 127.7 (C-4), 128.6 (C-11), 132.0 (C-12), 132.6 (C-3), 138.3 (C-6), 139.1 (C-10), $140.3(\mathrm{C}-9), 144.9(\mathrm{C}-5)$.

MS (EI): m/z $332.6\left(\mathrm{M}^{+}\right), 334.6\left(\mathrm{M}^{+}\right)$.

HR-MS (EI): calcd. for $\mathrm{C}_{19} \mathrm{H}_{12} \mathrm{Br}$ : 333.0153; found: 333.0152 .

1-(2,5-Dimethyl-1H-pyrrole)-4' '-nitro-4,1' $: 4{ }^{\prime}, 1^{\prime \prime}$ '-terphenylene (NOPYRP3) Under an argon atmosphere $620 \mathrm{mg}(1.9 \mathrm{mmol})$ of BRPYRP2, $350 \mathrm{mg}$ (2.0 mmol, 1.05 equivalents) of 4- nitrophenylboronic acid, $866 \mathrm{mg}$ (5.7 mmol, 3.0 equivalents) of cesium fluoride, and $66 \mathrm{mg}(0.057 \mathrm{mmol}, 3 \mathrm{~mol} \%)$ of $\left[\mathrm{Pd}\left(\mathrm{PPh}_{3}\right)_{4}\right]$ were dissolved in $50 \mathrm{~mL}$ of abs. THF and refluxed for 30 hours. After that time the reaction mixture was diluted with $50 \mathrm{~mL}$ of dichloromethane, washed with water, dried over sodium sulphate, and concentrated in vacuo. The crude product was purified by flash chromatography on silica using $n$-hexane/ethyl acetate/dichloromethane 10:5:1 + 5\% triethylamine as eluent to give 320 mg (0.87 mmol, 46\%) of NOPYRP3 as a brown amorphous solid.

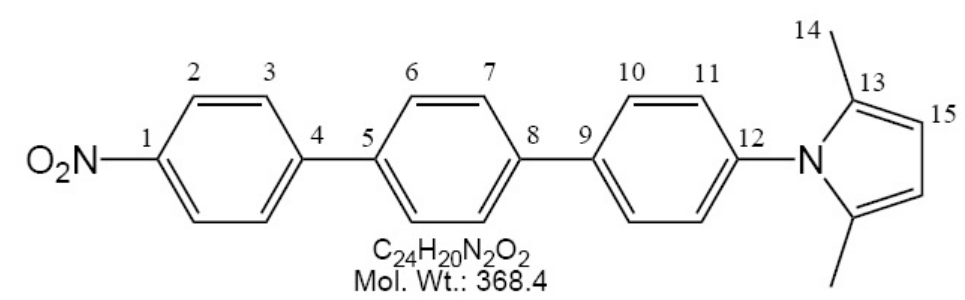

${ }^{1} \mathrm{H}-\mathrm{NMR}\left(\mathrm{CDCl}_{3}, \delta[\mathrm{ppm}]\right): 2.09(6 \mathrm{H}, \mathrm{s}, \mathrm{H}-14), 5.93(2 \mathrm{H}, \mathrm{s}, \mathrm{H}-15), 7.31(2 \mathrm{H}, \mathrm{d}, J=$ $8.2 \mathrm{~Hz}, \mathrm{H}-11), 7.72(2 \mathrm{H}, \mathrm{d}, J=8.2 \mathrm{~Hz}, \mathrm{H}-10), 7.75$ (4 H, d, J = 6.9 Hz, H-6, H-7), 7.80 $(2 \mathrm{H}, \mathrm{d}, J=8.8 \mathrm{~Hz}, \mathrm{H}-3), 8.32(2 \mathrm{H}, \mathrm{d}, J=8.8 \mathrm{~Hz}, \mathrm{H}-2)$.

${ }^{13} \mathrm{C}-\mathrm{NMR}\left(\mathrm{CDCl}_{3}, \delta[\mathrm{ppm}]\right): 13.1$ (C-14), 105.9 (C-13), 124.2, (C-2), 127.6 (2 C, C-6, C-7), 127.8 (C-3), 127.9 (C-10*), 128.9 (C-11*), 137.9 (C-9), 138.7 (C-5*), 139.4 (C-8*), 140.8 (C-12), 146.9 (C-4), $147.2(\mathrm{C}-1)$.

MS (EI): m/z $368.1\left(\mathrm{M}^{+}\right), 275.1\left(\left[\mathrm{M}-\mathrm{C}_{6} H_{7} N\right]^{+}\right)$.

HR-MS (EI): calcd. for $\mathrm{C}_{24} \mathrm{H}_{20} \mathrm{~N}_{2} \mathrm{O}_{2}$ : 368.1524; found: 368.1521 . 
1-lodo-4' '-nitro-4,1':4',1' '-terphenylene (NOIP3) [222] $320 \mathrm{mg}(0.87 \mathrm{mmol})$ of NOPYRP3 were dissolved in a mixtrure of $20 \mathrm{~mL}$ of $2 \mathrm{~N}$ sulphuric acid and $20 \mathrm{~mL}$ of acetonitrile and cooled down to $-5{ }^{\circ} \mathrm{C}$. At this temperature $180 \mathrm{mg}$ (2.6 mmol, 3 equivalents) of sodium nitrite dissolved in $5 \mathrm{~mL}$ of water were added dropwise. After stirring for 30 minutes $722 \mathrm{mg}$ ( $4.4 \mathrm{mmol}, 5$ equivalents) of potassium iodide dissolved in $5 \mathrm{~mL}$ of water were slowly added dropwise at $-5{ }^{\circ} \mathrm{C}$. After removing of the cooling bath, the reaction mixture was stirred for $1 \mathrm{~h}$, heated up to $60{ }^{\circ} \mathrm{C}$ shortly, and stirred at room temperature for additional $8 \mathrm{~h}$. The reaction mixture was neutralised with sat. aqu. $\mathrm{Na}_{2} \mathrm{CO}_{3}$. The aqueous layer was extracted repeatedly with dichloromethane. The combined organic layers were washed with water, a solution of sodium thiosulphate, and again water, dried over sodium sulphate, and concentrated in vacuo. The crude product was purified by flash chromatography on silica using first $n$-hexane/ethyl acetate 5:1 and then dichloromethane/ $n$-hexane $5: 1$ containing $5 \%$ of triethylamine as eluents to give 70 mg (0.17 mmol, 20\%) of NOIP3 as a yellow amorphous solid.

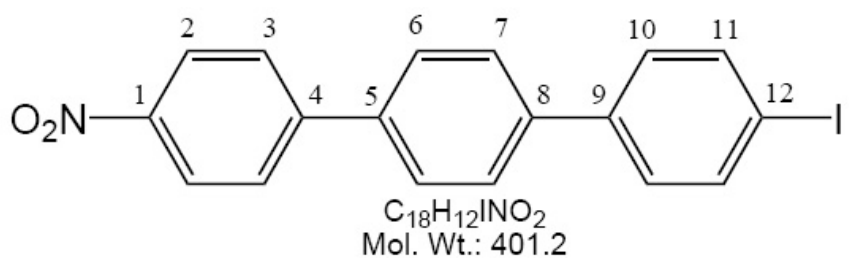

${ }^{1} \mathrm{H}-\mathrm{NMR}\left(\mathrm{CDCl}_{3}, \delta[\mathrm{ppm}]\right): 7.41(2 \mathrm{H}, \mathrm{d}, J=8.4, \mathrm{H}-10), 7.72\left(2 \mathrm{H}, \mathrm{d}, J=8.4, \mathrm{H}-7^{*}\right)$, $7.75\left(2 \mathrm{H}, \mathrm{d}, J=8.8, \mathrm{H}-6^{*}\right), 7.82(2 \mathrm{H}, \mathrm{d}, J=8.8, \mathrm{H}-3), 7.84(2 \mathrm{H}, \mathrm{d}, J=8.4, \mathrm{H}-11)$ $8.36(2 \mathrm{H}, \mathrm{d}, J=8.8, \mathrm{H}-2)$.

${ }^{13} \mathrm{C}-\mathrm{NMR}\left(\mathrm{CDCl}_{3}, \delta[\mathrm{ppm}]\right): 93.6(\mathrm{C}-12), 124.2(\mathrm{C}-2), 127.5\left(\mathrm{C}-6^{*}\right), 127.6\left(\mathrm{C}-7^{*}\right), 127.9$ (C-11), 128.8 (C-10), 138.0 (C-3, C-9), 139.6 (C-5), 140.6 (C-8), 145.6 (C-4), 146.9 (C-1). MS (EI): m/z $401.0\left(\mathrm{M}^{+}\right)$.

HR-MS (EI): calcd. for $\mathrm{C}_{18} \mathrm{H}_{12} \mathrm{INO}_{2}$ : 400.9913; found: 400.9914.

\section{1-Chloro-4' ' '-methoxy-4,1' $: 4^{\prime}, 1^{\prime \prime}: 4^{\prime}{ }^{\prime}, 1^{\prime \prime}$ ' '$^{\prime}$-quaterphenylene (MOCLP4)}

Under an argon atmosphere $300 \mathrm{mg}(0.9 \mathrm{mmol})$ of MOBRP3, $142 \mathrm{mg}$ (0.91 mmol, 1.2 equivalents) of 4-chlorophenylboronic acid, $347 \mathrm{mg}$ (2.23 mmol, 3 equivalents) of cesium fluoride, and $43 \mathrm{mg}(0.058 \mathrm{mmol}, 5 \mathrm{~mol} \%)$ of $\left[\mathrm{Pd}\left(\mathrm{PPh}_{3}\right)_{4}\right]$ were dissolved in $60 \mathrm{~mL}$ of abs. THF and refluxed for 50 hours. The product precipitated from the reaction mixture and was collected by filtration. The crude product was washed repeatedly with tetrahydrofurane and water to give $260 \mathrm{mg}(0.70 \mathrm{mmol}, 92 \%)$ of MOCLP4 as an off-white amorphous solid.

${ }^{1} \mathrm{H}-\mathrm{NMR}\left(\mathrm{CDCl}_{3}, \delta[\mathrm{ppm}]\right): 3.86(6 \mathrm{H}, \mathrm{s}, \mathrm{H}-1), 6.99(2 \mathrm{H}, \mathrm{d}, J=8.3 \mathrm{~Hz}, \mathrm{H}-3), 7.42(2 \mathrm{H}$, 


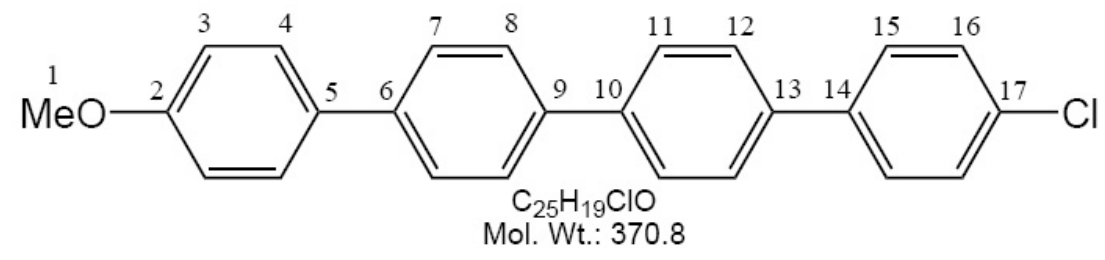

d, $J=8.1 \mathrm{~Hz}, \mathrm{H}-16), 7.55-7.60$ (4 H, m, H-4, H-15), 7.62 - 7.66 (4 H, m, H-7*, H-12*), $7.67-7.72\left(4 \mathrm{H}, \mathrm{m}, \mathrm{H}-8^{*}, \mathrm{H}-11^{*}\right)$.

MS (CI, isobutane): $\mathrm{m} / \mathrm{z} 371.1\left(\mathrm{M}+\mathrm{H}^{+}\right)$.

HR-MS (EI): calcd. for $\mathrm{C}_{25} \mathrm{H}_{19} \mathrm{ClO}$ : 370.1124; found: 370.1124 .

UVvis (dichloromethane): $\lambda_{\max }=309 \mathrm{~nm}$.

\section{1-Amino-4' '-methoxy-4,1 ' $^{\prime} 4^{\prime}, 1^{\prime \prime}: 4^{\prime \prime}, 1^{\prime \prime}{ }^{\prime \prime}$-quaterphenylene (MONHP4)}

Under an argon atmosphere $577 \mathrm{mg}(1.70 \mathrm{mmol})$ of MOBRP3, $575 \mathrm{mg}$ (1.80 mmol, 1.05 equivalents) of tert-butyl- 4- (4,4,5,5-tetramethyl-1,3,2-dioxaborolan-2-yl)phenylcarbamate, $800 \mathrm{mg}$ (5.3 mmol, 3 equivalents) of cesium fluoride, and $57 \mathrm{mg}(0.05 \mathrm{mmol}, 3 \mathrm{~mol} \%)$ of $\left[\mathrm{Pd}\left(\mathrm{PPh}_{3}\right)_{4}\right]$ were dissolved in $50 \mathrm{~mL}$ of abs. THF and refluxed for 50 hours. The Bocprotected product precipitated from the reaction mixture, was collected by filtration after cooling to room temperature, and washed repeatedly with tetrahydrofurane and water to give a light yellow amorphous solid. This was suspended in dichloromethane and cooled to $0{ }^{\circ} \mathrm{C}$. Then $6 \mathrm{~mL}$ of trifluoroacetic acid were added dropwise and the resulting solution was stirred for 12 hours at room temperature. Upon treatment with ca. $8 \mathrm{~mL}$ of $2 \mathrm{M}$ aqueous sodium hydroxide the product precipitated from the solution, was collected by filtration, and washed repeatedly with water and dichloromethane to give $537 \mathrm{mg}$ (1.53 mmol, $90 \%$ ) of MONHP4 as a fawn amorphous solid.

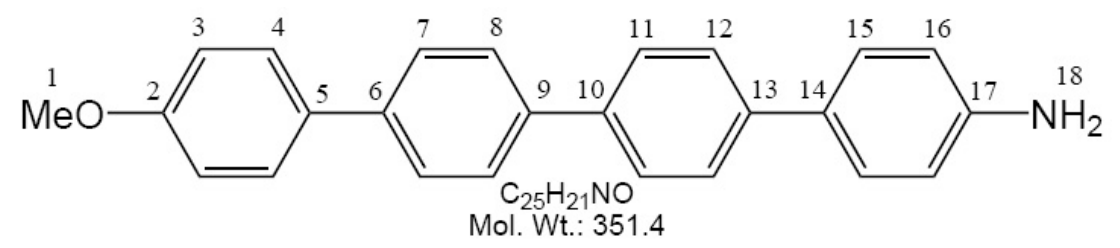

${ }^{1} \mathrm{H}-\mathrm{NMR}\left(\mathrm{CDCl}_{3}\right.$ :trifluoro acetic acid 3:1, $\left.\delta[\mathrm{ppm}]\right): 4.00(3 \mathrm{H}, \mathrm{s}, \mathrm{H}-1), 7.10(2 \mathrm{H}, \mathrm{d}, J=$ $8.5 \mathrm{~Hz}, \mathrm{H}-3), 7.48$ (2 H, d, $J=8.3 \mathrm{~Hz}, \mathrm{H}-15), 7.64(2 \mathrm{H}, \mathrm{d}, J=8.5 \mathrm{~Hz}, \mathrm{H}-4), 7.67$ (4 H, $\left.\mathrm{d}, J=8.2 \mathrm{~Hz}, \mathrm{H}-8^{*}, \mathrm{H}-11^{*}\right), 7.73\left(2 \mathrm{H}, \mathrm{d}, J=8.2 \mathrm{~Hz}, \mathrm{H}-7^{*}\right), 7.78(2 \mathrm{H}, \mathrm{d}, J=8.2 \mathrm{~Hz}$, H-12*), 7.81 (2 H, d, $J=8.3 \mathrm{~Hz}, \mathrm{H}-16), 8.73$ (3 H, broad s, H-18).

MS (EI): m/z $351.1\left(\mathrm{M}^{+}\right)$.

HR-MS (EI): calcd. for $\mathrm{C}_{25} \mathrm{H}_{21} \mathrm{NO}$ : 351.1623; found: 351.1622 .

UVvis (dichloromethane): $\lambda_{\max }=317 \mathrm{~nm}$.

UVvis (dichloromethane:trifluoro acetic acid 10:1): $\lambda_{\max }=307 \mathrm{~nm}$. 


\section{1-Cyano-4' ' -methoxy-4,1' $: 4{ }^{\prime}, 1^{\prime \prime}: 4^{\prime \prime}, 11^{\prime \prime}$ ' -quaterphenylene (MOCNP4)}

Under an argon atmosphere $851 \mathrm{mg}(2.5 \mathrm{mmol})$ of MOBRP3, $393 \mathrm{mg}$ (2.7 mmol, 1.1 equivalents) of 4-cyanophenylboronic acid, $1.14 \mathrm{~g}$ (7.5 mmol, 3.0 equivalents) of cesium fluoride, and $86 \mathrm{mg}(0.116 \mathrm{mmol}, 4 \mathrm{~mol} \%)\left[\mathrm{Pd}\left(\mathrm{PPh}_{3}\right)_{4}\right]$ were dissolved in $60 \mathrm{~mL}$ of abs. THF and refluxed for 50 hours. The product precipitated from the reaction mixture and was collected by filtration. The crude product was washed repeatedly with THF and water to give $867 \mathrm{mg}(2.4 \mathrm{mmol}, 96 \%)$ of MOCNP4 as a grey amorphous solid.

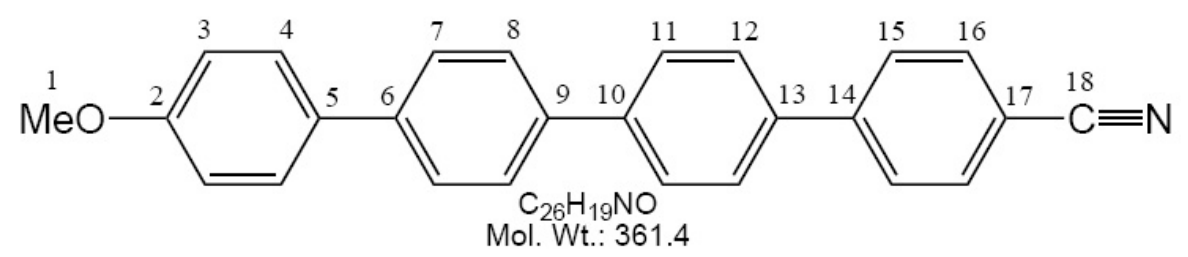

${ }^{1} \mathrm{H}-\mathrm{NMR}\left(\mathrm{CDCl}_{3}, \delta[\mathrm{ppm}]\right): 3.86(3 \mathrm{H}, \mathrm{s}, \mathrm{H}-1), 7.00(2 \mathrm{H}, \mathrm{d}, J=8.6 \mathrm{~Hz}, \mathrm{H}-3), 7.58(2 \mathrm{H}$, $\mathrm{d}, J=8.6 \mathrm{~Hz}, \mathrm{H}-4), 7.63-7.71(6 \mathrm{H}, \mathrm{m}), 7.73-7.77$ (6 H, d, $J$ not resolved).

MS (ESI, positive): $\mathrm{m} / \mathrm{z} 362.3\left(\mathrm{M}^{+}\right)$.

HR-MS (EI): calcd. for $\mathrm{C}_{26} \mathrm{H}_{19} \mathrm{NO}$ : 361.1466; found: 361.1467 .

UVvis (dichloromethane): $\lambda_{\max }=317 \mathrm{~nm}$.

\subsubsection{Symmetrically functionalised para-Hexaphenylenes}

\section{4- 4'''-Dimethoxy-p-hexaphenylene (MOP6)}

Under an argon atmosphere 4,4'-biphenyldiboronic acid bis(neopentyl glycol)ester (1.0 mmol), 4-iodo,4'-methoxy biphenyl (MOIP2) (2.2 mmol, 2.2 equivalents), cesium fluoride (4.0 mmol, 4.0 equivalents) and tetrakis(triphenylphosphine)-palladium(0) (0.05 mmol, 5 mol\%) were dissolved in $80 \mathrm{~mL}$ tetrahydrofurane abs. and refluxed for 70 hours. After cooling to room temperature the precipitate was collected by filtration. The crude product was washed repeatedly with dichloromethane and water to give $518 \mathrm{mg}$ (1.0 mmol, quant.) of a slightly coloured amorphous solid.

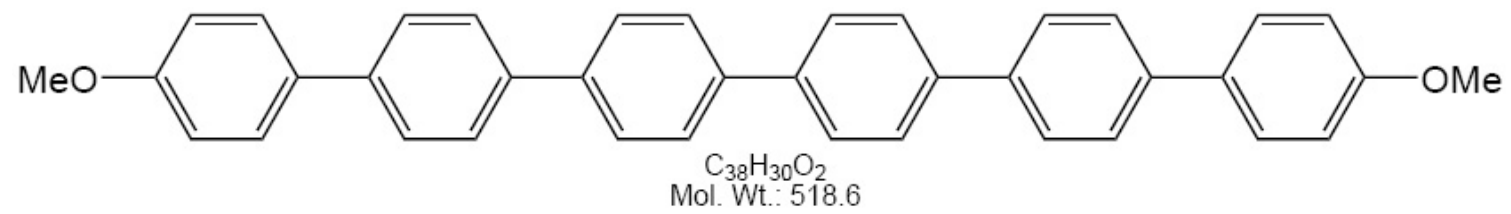

MS (EI): m/z $518.6\left(\mathrm{M}^{+}\right), 411.7\left([\mathrm{M}-\mathrm{PhOMe}]^{+}\right), 258.8\left(\mathrm{M}^{2+}\right)$. 
HR-MS (EI): calcd. for $\mathrm{C}_{38} \mathrm{H}_{30} \mathrm{O}_{2}$ : 518.2246; found: 518.2246.

UVvis (dichloromethane): $\lambda_{\max }=317 \mathrm{~nm}$.

\section{4- 4'"' 'Dichloro-p-hexaphenylene (CLP6)}

Under argon atmosphere 4,4'-biphenyldiboronic acid bis(neopentyl glycol)ester (0.5 mmol), 4-chloro,4'-iodo biphenyl (CLIP2) (1.1 mmol, 2.2 equivalents), cesium fluoride (2.0 mmol, 4.0 equivalents) and tetrakis(triphenylphosphine)-palladium(0) (0.025 mmol, $5 \mathrm{~mol} \%$ ) were dissolved in $60 \mathrm{~mL}$ tetrahydrofurane abs. and refluxed for 70 hours. After cooling to room temperature the precipitate was collected by filtration. The crude product was washed with dichloromethane and water to give $204 \mathrm{mg}$ (1.0 mmol, quant.) of a slightly coloured amorphous solid.

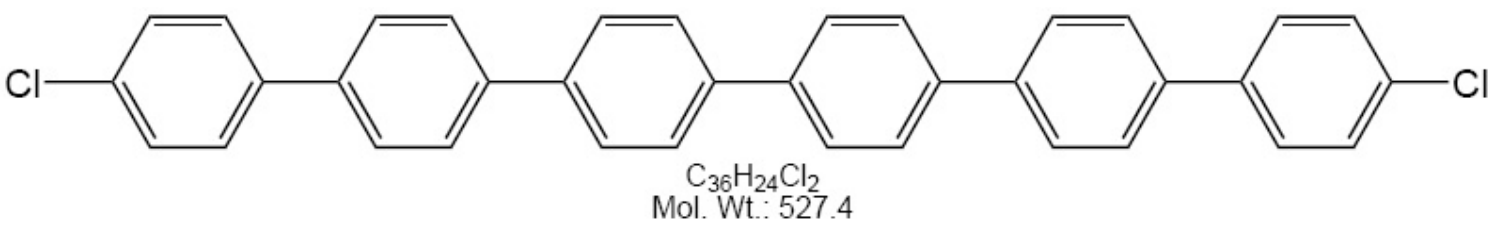

MS (EI): m/z 525.5 $\left(\mathrm{M}^{+}\right), 527.5\left(\mathrm{M}^{+}\right), 415.7\left([\mathrm{M}-\mathrm{PhCL}]^{+}\right), 262.8\left(\mathrm{M}^{2+}\right), 263.8\left(\mathrm{M}^{2+}\right)$. HR-MS (EI): calcd. for $\mathrm{C}_{36} \mathrm{H}_{24} \mathrm{Cl}_{2}$ : 526.1255; found: 526.1254 .

UVvis (dichloromethane): $\lambda_{\max }=313 \mathrm{~nm}$.

\section{4- 4'"' 'Dicyano-p-hexaphenylene (CNP6)}

Under an argon atmosphere 4,4'-biphenyldiboronic acid bis(neopentyl glycol)ester (0.5 mmol), 4-cyano,4'-iodo biphenyl (CNIP2) (1.1 mmol, 2.2 equivalents), cesium fluoride (2.0 mmol, 4.0 equivalents) and tetrakis(triphenylphosphine)-palladium(0) (0.025 mmol, $5 \mathrm{~mol} \%$ ) were dissolved in $60 \mathrm{~mL}$ tetrahydrofurane abs. and refluxed for 70 hours. After cooling to room temperature the precipitate was collected by filtration. The crude product was washed repeatedly with dichloromethane and water to give $204 \mathrm{mg}$ (1.0 mmol, quant.) of a slightly coloured amorphous solid.

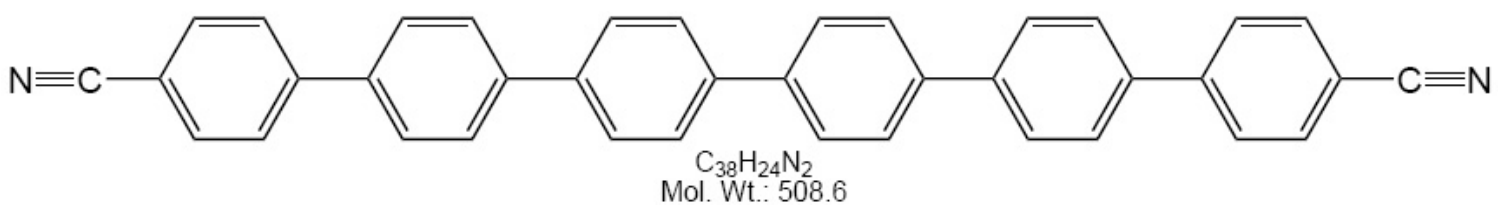

MS (EI): m/z $507.6\left(\mathrm{M}^{+}\right), 406.7\left([\mathrm{M}-\mathrm{PhCN}]^{+}\right), 253.8\left(\mathrm{M}^{2+}\right)$.

HR-MS (EI): calcd. for $\mathrm{C}_{38} \mathrm{H}_{24} \mathrm{~N}_{2}$ : 508.1939; found: 508.1939 .

UVvis (dichloromethane): $\lambda_{\max }=332 \mathrm{~nm}$. 


\subsubsection{Phenyl-thiophene co-oligomers}

\section{4,4'-Di-2,2'-bithienyl-biphenyl (TTPPTT) [81]}

Under an argon atmosphere 4,4'-biphenyldiboronic acid bis(neopentyl glycol)ester (719 mg, $1.9 \mathrm{mmol}$ ), 5-bromo-2,2'-bithiophene (1.00 g, $4.08 \mathrm{mmol}, 2.1$ equivalents), tetrakis(triphenylphosphine)-palladium(0) (109 $\mathrm{mg}, 0.095 \mathrm{mmol}, 5 \mathrm{~mol} \%$ ) and cesium fluoride (1.16 g, $7.6 \mathrm{mmol}, 4$ equivalents) were dissolved in $80 \mathrm{~mL}$ tetrahydrofurane abs. and refluxed for 50 hours. After cooling to room temperature the reaction mixture was diluted with $n$-hexane, and the precipitate was collected by filtration. The crude product was washed with water, ethyl acetate and dichloromethane to give $830 \mathrm{mg}$ (1.72 mmol, 91\%) of a bright yellow amorphous solid.

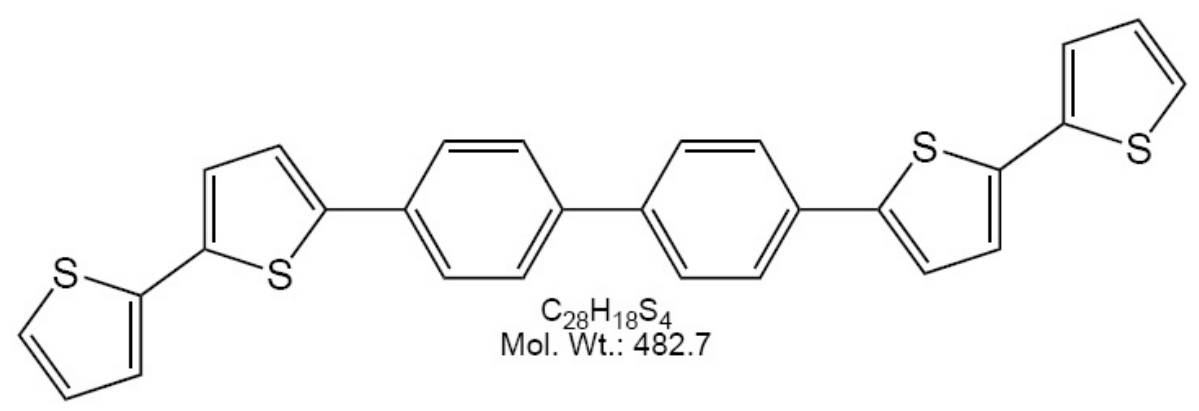

MS (EI): m/z $482.0\left(\mathrm{M}^{+}\right), 241.0\left(\mathrm{M}^{2+}\right)$.

HR-MS (EI): calcd. for $\mathrm{C}_{28} \mathrm{H}_{18} \mathrm{~S}_{4}$ : 482.0291; found: 482.0283 .

UVvis (dichloromethane): $\lambda_{\max }=271 \mathrm{~nm}, 380 \mathrm{~nm}$.

\section{5,5'-Di-4-biphenyl-2,2'-bithiophene (PPTTPP) [223]}

Under an argon atmosphere 5,5'-dibromo-2,2'-bithiophene (500 mg, $1.54 \mathrm{mmol}$ ), 4-biphenyl boronic acid (627 mg, $3.16 \mathrm{mmol}, 2.05$ equivalents), tetrakis(triphenylphosphine)palladium(0) (88 mg, $0.077 \mathrm{mmol}, 5 \mathrm{~mol} \%$ ) and cesium fluoride (702 mg, $4.6 \mathrm{mmol}, 3$ equivalents) were dissolved in $80 \mathrm{~mL}$ tetrahydrofurane abs. and refluxed for 50 hours. After cooling to room temperature the reaction mixture was diluted with $n$-hexane, and the precipitate was collected by filtration. The crude product was washed with water, ethyl acetate and dichloromethane to give $692 \mathrm{mg}(1.47 \mathrm{mmol}, 95 \%)$ of a yellow-orange amorphous solid.

MS (EI): m/z $470.1\left(\mathrm{M}^{+}\right), 235.0\left(\mathrm{M}^{2+}\right)$.

HR-MS (EI): calcd. for $\mathrm{C}_{32} \mathrm{H}_{22} \mathrm{~S}_{2}$ : 470.1162; found: 470.1162. 


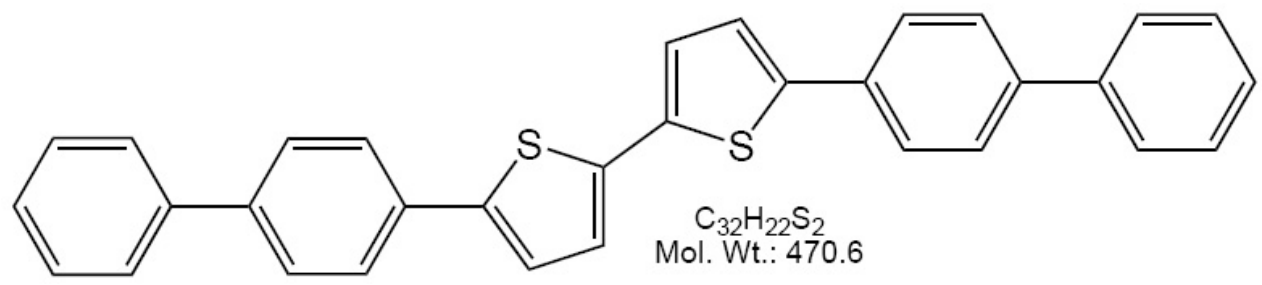

\subsubsection{Symmetrically functionalised Phenyl-ethinylene co-oligomers}

\section{4,4’-Bis(trimethylsilyl)biphenyl [224], [225]}

To a solution of 4,4'-dibrombiphenyl (4.0 mmol,1.25 g), $\mathrm{Pd}\left(\mathrm{PPh}_{3}\right)_{2} \mathrm{Cl}_{2}$ (0.12 mmol, 84 $\mathrm{mg}, 3 \mathrm{~mol} \%$ ), $\mathrm{PPh}_{3}$ (0.24 mmol, $\left.63 \mathrm{mg}, 6 \mathrm{~mol} \%\right)$, CuI (0.20 mmol, $\left.38 \mathrm{mg}, 5 \mathrm{~mol} \%\right)$ in 40 $\mathrm{mL}(i \mathrm{Pr})_{2} \mathrm{NH}(\mathrm{abs})$ under argon atmosphere were added slowly via syringe $1.7 \mathrm{~mL}(12.0$ mmol, 3 equivalents) trimethylsilyl acetylene. The solution was heated to $70^{\circ} \mathrm{C}$ for 12 hours. The solution was then filtered through celite, the celite was flushed with $n$-hexane and the filtrate was washed with water. The solvent was evaporated and the residue was purified by silica gel chromatography ( $n$-hexane $+0.5 \%$ triethylamine) to give $1.2 \mathrm{~g}(3.47$ mmol, $86 \%$ ) of a microcristalline white solid.

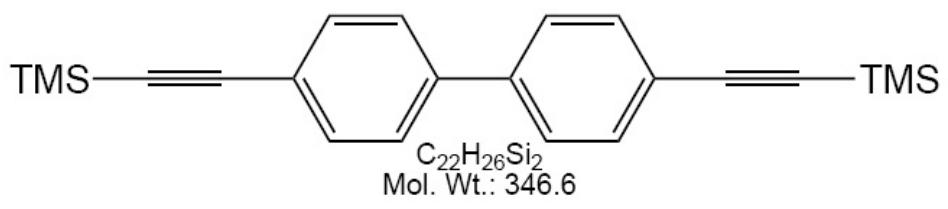

${ }^{1} \mathrm{H}-\mathrm{NMR}\left(\mathrm{CDCl}_{3}, \delta[\mathrm{ppm}]\right): 0.26(\mathrm{~s}, 18 \mathrm{H}), 7.52(\mathrm{~s}, 8 \mathrm{H})$

${ }^{13} \mathrm{C}-\mathrm{NMR}\left(\mathrm{CDCl}_{3}, \delta[\mathrm{ppm}]\right): 0.0$ (6 C), 95.2 (2 C), 104.8 (2 C), 122.4 (2 C), 126.7 (4 C), 132.4 (4 C), 140.2 (2 C).

MS (CI, isobutane): $\mathrm{m} / \mathrm{z} 347.3\left(\mathrm{M}+\mathrm{H}^{+}\right)$.

\section{4,4'-Diethinylbiphenyl [224],[225]}

Potassium carbonate ( $2.4 \mathrm{~g}, 17.5 \mathrm{mmol}, 5$ equivalents) was added to a solution of 4,4'-(bistrimethylsilyl)biphenyl in $150 \mathrm{~mL}$ methanol. The reaction mixture was stirred at room temperature for 30 hours during which time the potassium carbonate dissolved. Water (200 mL) was added and the precipitate was filtered and washed with water to give 661 $\mathrm{mg}(3.3 \mathrm{mmol}, 95 \%)$ of a fawn microcrystalline solid.

${ }^{1} \mathrm{H}-\mathrm{NMR}\left(\mathrm{CDCl}_{3}, \delta[\mathrm{ppm}]\right): 3.14(\mathrm{~s}, 2 \mathrm{H}), 7.53(\mathrm{~d}, 8 \mathrm{H}, \mathrm{J}=8.5), 7.56(\mathrm{~d}, 4 \mathrm{H}, \mathrm{J}=8.5)$. ${ }^{13} \mathrm{C}-\mathrm{NMR}\left(\mathrm{CDCl}_{3}, \delta[\mathrm{ppm}]\right): 78.1$ (2 C), $83.4(2 \mathrm{C}), 121.5(2 \mathrm{C}), 126.9(4 \mathrm{C}), 132.7(4 \mathrm{C})$, 140.5 ( $2 \mathrm{C})$. 


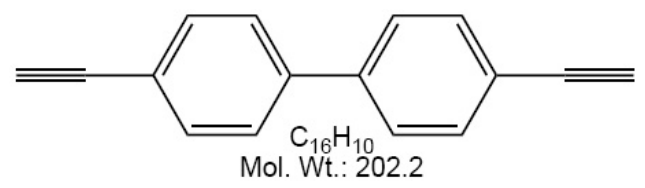

\section{4,4'-Bis((4-pyridyl)ethinyl)-1,1'-biphenyl (PYethinP2)}

Under argon atmosphere 4,4'-diethinylbiphenyl (330 mg, $1.63 \mathrm{mmol}$ ), 4-iodpyridine (PYI) (735 mg, $3.75 \mathrm{mmol}, 2.2$ equivalente), $\mathrm{Pd}\left(\mathrm{PPh}_{3}\right)_{2} \mathrm{Cl}_{2}$ (45 mg, $0.07 \mathrm{mmol}, 4 \mathrm{~mol} \%$ ) and copper(I)iodine (19 mg, $0.1 \mathrm{mmol}, 6 \mathrm{~mol} \%$ ) were dissolved in $40 \mathrm{~mL}$ tetrahydrofurane abs. and $10 \mathrm{~mL}$ dry triethylamine and heated up to $70^{\circ} \mathrm{C}$ for 30 hours. After cooling to room temperature the reaction mixture was diluted with $n$-hexane and the dark yellow precipitate was collected by filtration. The crude product was washed with $n$-hexane, tetrahydrofurane, aqueous ethylene diamine tetraacetic acid and water for purification to give $410 \mathrm{mg}$ (1.15 mmol, 71\%) of a dark yellow amorphous solid.

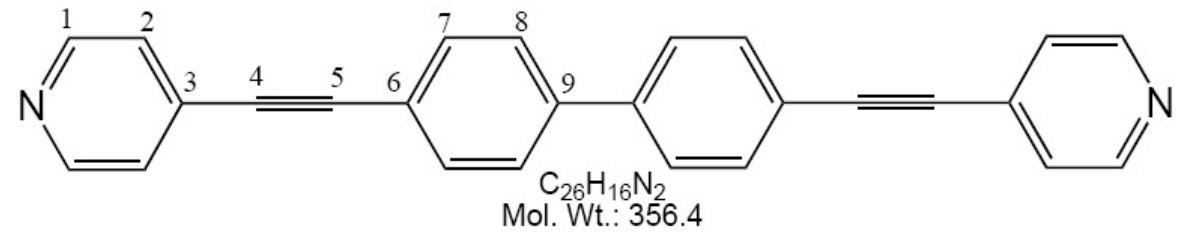

${ }^{1} \mathrm{H}-\mathrm{NMR}\left(\mathrm{CDCl}_{3}, \delta[\mathrm{ppm}]\right): 7.39(2 \mathrm{H}, \mathrm{d}, J=5.6 \mathrm{~Hz}, \mathrm{H}-2), 7.63(4 \mathrm{H}, \mathrm{s}, \mathrm{H}-7, \mathrm{H}-8), 8.61$ $(2 \mathrm{H}, \mathrm{d}, J=4.1 \mathrm{~Hz})$.

${ }^{13} \mathrm{C}-\mathrm{NMR}\left(\mathrm{CDCl}_{3}, \delta[\mathrm{ppm}]\right): 87.7(\mathrm{C}-4), 93.7$ (C-5), 121.6 (C-6), 125.5 (C-2), 127.1 (C-8), 131.4 (C-3), 132.5 (C-7), 140.8 (C-9), 149.8 (C-1).

MS (CI, isobutane): $\mathrm{m} / \mathrm{z} 357.0\left(\mathrm{M}+\mathrm{H}^{+}\right), 278.9\left(\left[\mathrm{M}-\mathrm{C}_{5} \mathrm{H}_{4} \mathrm{~N}+\mathrm{H}\right]^{+}\right)$.

HR-MS (EI): calcd. for $\mathrm{C}_{26} \mathrm{H}_{16} \mathrm{~N}_{2}$ : 356.1314; found: 356.1314 .

UVvis (dichloromethane): $\lambda_{\max }=328 \mathrm{~nm}$.

\section{4,4'-Bis((4-N,N-dimethylaminophenyl)ethinyl)-1,1'-biphenyl (NMePethinP2)}

Under argon atmosphere 4,4'diethinylbiphenyl (661 mg, $3.3 \mathrm{mmol}$ ), 4-bromo- $N, N$-dimethylaniline (1.38 g, $6.9 \mathrm{mmol}, 2.1$ equivalents), $\mathrm{Pd}\left(\mathrm{PPh}_{3}\right)_{2} \mathrm{Cl}_{2}$ (92 mg, $0.13 \mathrm{mmol}, 4$ mol\%), triphenyl phosphine (69 $\mathrm{mg}, 0.26 \mathrm{mmol}, 8 \mathrm{~mol} \%$ ) and copper(I)iodine (37 $\mathrm{mg}$, $0.20 \mathrm{mmol}, 6 \mathrm{~mol} \%$ ) were dissolved in $50 \mathrm{~mL}$ dry diisopropylamine and heated up to $70^{\circ} \mathrm{C}$ for 40 hours. Meanwhile the colour of the reaction mixture changed from yellow to dark brown. After cooling to room temperature a precipitate coloured like liquid manure was collected by filtration and washed repeadetly with $n$-hexane, dichloromethane, water and aqueous ethylene diamine tetraacetic acid without changing colour.

The crude product was suspended in about $100 \mathrm{~mL}$ dichloromethane and small portions trifluoro acetic was added until a dark green solution occurred. The black residues were 
removed by filtration quickly, because the product was corroding under these conditions. The filtrate was neutralised with $10 \mathrm{M}$ aqueous sodium hydroxide, meanwhile the colour of the solution turned over light green to yellow until occurrence of a precipitate. The product was collected by filtration and washed with water and dichloromethane to give $932 \mathrm{mg}$ (2.12 mmol, 64\%) of yellow amorphous solid.

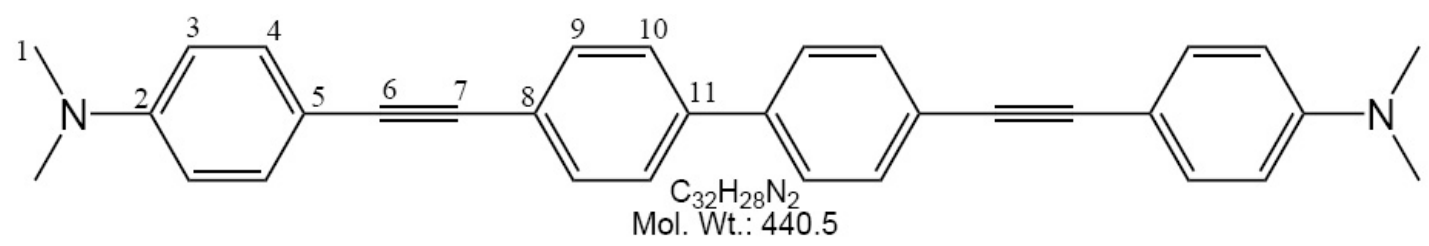

${ }^{1} \mathrm{H}-\mathrm{NMR}\left(\mathrm{CDCl}_{3}, \delta[\mathrm{ppm}]\right): 2.93(12 \mathrm{H}, \mathrm{s}, \mathrm{H}-1), 6.60(4 \mathrm{H}, \mathrm{d}, J=8.6 \mathrm{~Hz}, \mathrm{H}-3), 7.35$ (4 $\mathrm{H}, \mathrm{d}, J=8.6 \mathrm{~Hz}, \mathrm{H}-4), 7.50$ (8 H, s, H-9, H-10).

${ }^{1} \mathrm{H}-\mathrm{NMR}\left(\mathrm{CDCl}_{3}\right.$ :trifluoro acetic acid 4:1, $\left.\delta[\mathrm{ppm}]\right): 3.35(12 \mathrm{H}, \mathrm{s}, \mathrm{H}-1), 7.48(4 \mathrm{H}, \mathrm{d}, J=$ $8.6 \mathrm{~Hz}, \mathrm{H}-3), 7.64$ (8 H, s, H-9, H-10), 7.74 (4 H, d, J = 8.6 Hz, H-4), 10.22 (2 H, broad $\mathrm{s}$, protonated $\left.-\mathrm{N}(\mathrm{Me})_{2}\right)$.

${ }^{13} \mathrm{C}-\mathrm{NMR}\left(\mathrm{CDCl}_{3}\right.$ :trifluoro acetic acid 4:1, $\left.\delta[\mathrm{ppm}]\right): 47.4(\mathrm{C}-1), 87.4(\mathrm{C}-6), 92.8(\mathrm{C}-7)$, 119.9 (C-3), 121.6 (C-8), 127.2 (C-10), 127.5 (C-5), 132.5 (C-9), 134.1 (C-4), 140.6 (C-2), $141.0(\mathrm{C}-11)$.

MS (EI): m/z $440.2\left(\mathrm{M}^{+}\right), 425.2\left(\left[\mathrm{M}-\mathrm{CH}_{3}\right]^{+}\right), 220.1\left(\mathrm{M}^{2+}\right)$.

HR-MS (EI): calcd. for $\mathrm{C}_{32} \mathrm{H}_{28} \mathrm{~N}_{2}$ : 440.2252; found: 440.2252 .

UVvis (dichloromethane): $\lambda_{\max }=362 \mathrm{~nm}$. 
7 Experimental 


\section{Conclusions and Outlook}

Coming back to the initial question: Is the combination of $p$-hexaphenylene as molecular building block and muscovite mica as growth substrate really unique for generating the nanofibers? No, it is not. According to this in the context of this thesis the following results have been presented:

- A reliable synthesis strategy to obtain new either symmetrically or non-symmetrically para-functionalised para-quaterphenylenes.

- The possibility of well defined nanofiber growth starting from the substituted oligomers, i.e. fiber growth is not prevented by the functional groups but improved in some cases, on a muscovite mica substrate by a self-assembly process in high vacuum.

- The distinctive properties of the nanostructures are determined by the respective functional groups attached to the molecular building block:

- The exact wavelength of the fluorescence colour shifts within the blue depending on the functional group.

- The individual morphology, i.e. cross-section shape is defined by the functional group.

- New properties like non-linear optical activity (frequency doubling) of the nanofibers are created due to intrinsic non-zero hyperpolaisability of the non-symmetrically substituted molecular building blocks.

Thus, it is possible to obtain tailored nanofibers from appropriatly functionalised $p$ quaterphenylenes. The requirements for generating nanofibers seem to be still muscovite mica as growth substrate, but the molecular basis is not restricted to $\mathrm{p} 6 \mathrm{P}$ but to a paraphenylene molecular basis, which tolerates functional groups at the para-positions.

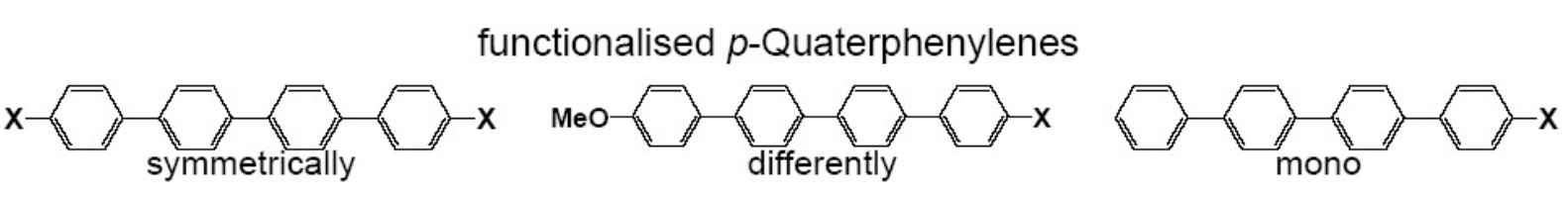

Fig. 8.1: Schematic molecular formulas of inspected functionalised $p$-Quaterphenylenes. Declaration of functional group X and short names are listed in Tab.8.1. 


\begin{tabular}{c|ccc}
\hline & \multicolumn{3}{|c}{ funct. p4P } \\
functional group X & symmetrically & differently & mono \\
\hline methoxy (-OMe) & MOP4 & - & MOHP4 \\
chloride (-Cl) & CLP4 & MOCLP4 & CLHP4 \\
cyano (-CN) & CNP4 & MOCNP4 & CNHP4 \\
amino (-NH & NHP4 & MONHP4 & - \\
$N, N$-dimethyl amino $\left(-\mathrm{NMe}_{2}\right)$ & NMeP4 & - & NMeHP4 \\
nitro $\left(-\mathrm{NO}_{2}\right)$ & NOP4 & - & - \\
\hline
\end{tabular}

Tab. 8.1: List of inspected functionalised $p$-Quaterphenylenes. Functional groups X refer to the schematic molecular formulas of Fig.8.1.

The new oligomers have been vapour deposited on a freshly cleaved muscovite mica substrate in high vacuum. The driving force of the self-assembly into well defined nanostructures is dipole assisted alignment and epitaxy.

A certain pre-definition of the nanostructures' properties is given by the functional groups, but still the growth conditions allow control over the nanostructures' morphology. The optical properties are basicly determined by the functional groups. Intermolecular interactions of the bulk structure (the nanostructures) are more pronounced for the mono-functionalised $p$-phenylenes than for the disubstituted oligomers. Properties are not simply given by the functional group, which makes a reliable forecast difficult (see Tab. 8.2, 8.3, 8.4). However, intrinsic properties of the oligomers are maintained in the respective nanostructures, such as the inherent non-zero hyperpolarisability of the nonsymmetrically substituted oligomers results in non-linear optical activity of the nanofibers. The individual morphology (cross-section shape) of the nanostructures is depending on the functional groups, but dimensions (length, height, width, aspect ratio) and aggregate density are mainly controlled by the growth conditions. Nevertheless, the fibers possess individual average length according to the functional group (short: few micrometer, medium: several ten micrometer, long: few hundred micrometer, very long: several hundred micrometer, cf. Tab. 8.2, 8.3, 8.4. An important role plays the substrate temperature during depositions: For some functionalities two morphologically different structure types (two different temperature cases) exist for distinct substrate temperatures.

The desired nanofibers are built up of lying molecules on the surface ordered with a high degree of crystallinity. Clusters are located in between the nanofibers, which reflect the bottom-up fashion of the growth process, since they are believed to be needle precursors. In addition islands of upright standing oligomers form on the surface in competition to nanofiber growth. The microscopic steps of the formation of clusters and the transformation of those into nanofibers are not resolved yet, and has to be clarified in the future by in situ microscopy. This may allow a better forecast of nanofiber growth 


\begin{tabular}{|c|c|c|c|c|c|c|}
\hline $\begin{array}{c}\text { molecular } \\
\text { building block }\end{array}$ & MOP4 & CLP4 & CNP4 & NMeP4 & NHP4 & NOP4 \\
\hline polarised fluorescence & $\sqrt{ }$ & $\sqrt{ }$ & $\sqrt{ }$ & $\sqrt{ }$ & - & - \\
\hline maximum emission [nm] & 400 & 383 & 404 & 452 & 439 & - \\
\hline frequency doubling & - & - & - & - & - & - \\
\hline distinct temp. dependence & - & $\sqrt{ }$ & - & $\sqrt{ }$ & - & - \\
\hline inverted temp. behaviour & - & - & $\sqrt{ }$ & $\sqrt{ }$ & - & - \\
\hline $\begin{array}{c}\text { growth along: } \\
\text { grooved }\left\langle\begin{array}{lll}1 & 1 & 0\end{array}\right. \\
\text { non-grooved }\left\langle\begin{array}{lll}1 & 1 & 0\rangle\end{array}\right. \\
{\left[\begin{array}{lll}1 & 0 & 0\end{array}\right]}\end{array}$ & $\begin{array}{l}\sqrt{ } \\
- \\
- \\
\end{array}$ & $\begin{array}{l}\sqrt{ } \\
- \\
- \\
\end{array}$ & $\begin{array}{l}\sqrt{ } \\
\sqrt{ } \\
\sqrt{ }\end{array}$ & $\begin{array}{l}\sqrt{ } \\
- \\
- \\
\end{array}$ & $\begin{array}{l}\sqrt{ } \\
- \\
-\end{array}$ & $\begin{array}{l}\sqrt{ } \\
- \\
- \\
\end{array}$ \\
\hline $\begin{array}{c}\text { cross-section shape: } \\
\text { rounded } \\
\text { slab-like } \\
\text { pitched roof }\end{array}$ & $\begin{array}{l}- \\
\sqrt{ } \\
-\end{array}$ & $\begin{array}{l}- \\
- \\
\sqrt{ }\end{array}$ & $\begin{array}{l}- \\
- \\
\sqrt{ }\end{array}$ & $\begin{array}{l}\sqrt{ } \\
\sqrt{ } \\
-\end{array}$ & $\begin{array}{l}\sqrt{ } \\
- \\
- \\
\end{array}$ & $\begin{array}{l}\mathrm{n} / \mathrm{a} \\
\mathrm{n} / \mathrm{a} \\
\mathrm{n} / \mathrm{a}\end{array}$ \\
\hline $\begin{array}{c}\text { fiber shape: } \\
\text { straight } \\
\text { bent } \\
\text { kinks } \\
\text { branches } \\
\text { general description: }\end{array}$ & $\begin{array}{l}\sqrt{ } \\
- \\
\sqrt{ } \\
-\end{array}$ & $\begin{array}{l}\sqrt{ } \\
- \\
- \\
-\end{array}$ & $\begin{array}{c}\sqrt{ } \\
\sqrt{ } \\
- \\
- \\
\text { swallow } \\
\text { wings }\end{array}$ & $\begin{array}{c}- \\
\sqrt{ } \\
- \\
- \\
\text { worms, } \\
\text { pollywogs, } \\
\text { flakes }\end{array}$ & $\begin{array}{l}- \\
\sqrt{ } \\
- \\
-\end{array}$ & $\begin{array}{c}- \\
- \\
\sqrt{ } \\
- \\
\text { lego- } \\
\text { fibers }\end{array}$ \\
\hline fibers taper off & - & - & - & - & - & - \\
\hline fiber length & very long & medium & short & short & short & medium \\
\hline
\end{tabular}

Tab. 8.2: Tailoring nanofibers: The influence of molecular building blocks's substituents - symmetrically functionalised $p$-quaterphenylenes. 


\begin{tabular}{|c|c|c|c|}
\hline $\begin{array}{c}\text { molecular } \\
\text { building block }\end{array}$ & MONHP4 & MOCLP4 & MOCNP4 \\
\hline $\begin{array}{c}\text { polarised fluorescence } \\
\text { maximum emission [nm] }\end{array}$ & $\begin{array}{c}- \\
438\end{array}$ & $\begin{array}{c}\sqrt{ } \\
428\end{array}$ & $\begin{array}{c}\sqrt{ } \\
433\end{array}$ \\
\hline frequency doubling & $\sqrt{ }$ & $\sqrt{ }$ & $\sqrt{ }$ \\
\hline distinct temp. dependence & - & $\sqrt{ }$ & - \\
\hline inverted temp. behaviour & - & - & - \\
\hline $\begin{array}{c}\text { growth along: } \\
\text { grooved }\left\langle\begin{array}{lll}1 & 1 & 0\rangle\end{array}\right. \\
\text { non-grooved }\left\langle\begin{array}{lll}1 & 1 & 0\rangle\end{array}\right. \\
{\left[\begin{array}{lll}1 & 0 & 0\end{array}\right]}\end{array}$ & $\begin{array}{l}\sqrt{ } \\
- \\
-\end{array}$ & $\begin{array}{l}\sqrt{ } \\
- \\
-\end{array}$ & $\begin{array}{l}\sqrt{ } \\
- \\
-\end{array}$ \\
\hline $\begin{array}{c}\text { cross-section shape: } \\
\text { rounded } \\
\text { slab-like } \\
\text { pitched roof }\end{array}$ & $\begin{array}{l}\sqrt{ } \\
- \\
-\end{array}$ & $\begin{array}{l}\sqrt{ } \\
- \\
-\end{array}$ & $\begin{array}{l}\sqrt{ } \\
- \\
-\end{array}$ \\
\hline $\begin{array}{c}\text { fiber shape: } \\
\text { straight } \\
\text { bent } \\
\text { kinks } \\
\text { branches } \\
\text { general description: }\end{array}$ & $\begin{array}{c}- \\
\sqrt{ } \\
- \\
- \\
\text { worms }\end{array}$ & $\begin{array}{c}\sqrt{ } \\
- \\
\sqrt{ } \\
\sqrt{ } \\
\text { branches, } \\
\text { lego-fibers }\end{array}$ & $\begin{array}{c}\sqrt{ } \\
\sqrt{ } \\
- \\
- \\
\text { walking sticks, } \\
\text { fibers }\end{array}$ \\
\hline fibers taper off & $\sqrt{ }$ & - & $(\sqrt{ })$ \\
\hline fiber length & short & medium & long \\
\hline
\end{tabular}

Tab. 8.3: Tailoring nanofibers: The influence of molecular building blocks's substituents - differently functionalised $p$-quaterphenylenes. 


\begin{tabular}{|c|c|c|c|c|}
\hline $\begin{array}{c}\text { molecular } \\
\text { building block }\end{array}$ & MOHP4 & CLHP4 & NMeHP4 & CNHP4 \\
\hline $\begin{array}{c}\text { polarised fluorescence } \\
\text { maximum emission }[\mathrm{nm}]\end{array}$ & $\begin{array}{c}\sqrt{ } \\
397\end{array}$ & $\begin{array}{c}\sqrt{ } \\
390\end{array}$ & $\begin{array}{c}\sqrt{ } \\
440\end{array}$ & $\begin{array}{c}\sqrt{ } \\
417\end{array}$ \\
\hline frequency doubling & $\sqrt{ }$ & $\sqrt{ }$ & $\sqrt{ }$ & $\sqrt{ }$ \\
\hline distinct temp. dependence & $\mathrm{n} / \mathrm{a}$ & $\mathrm{n} / \mathrm{a}$ & $\mathrm{n} / \mathrm{a}$ & - \\
\hline inverted temp. behaviour & $\mathrm{n} / \mathrm{a}$ & $\mathrm{n} / \mathrm{a}$ & $\mathrm{n} / \mathrm{a}$ & - \\
\hline $\begin{array}{c}\text { growth along: } \\
\text { grooved }\left\langle\begin{array}{lll}1 & 1 & 0\rangle \\
\text { non-grooved }\langle 1 & 1 & 0\rangle \\
{\left[\begin{array}{lll}1 & 0 & 0\end{array}\right]}\end{array}\right.\end{array}$ & $\begin{array}{l}\sqrt{ } \\
- \\
-\end{array}$ & $\begin{array}{l}\sqrt{ } \\
- \\
-\end{array}$ & $\begin{array}{l}\sqrt{ } \\
- \\
-\end{array}$ & $\begin{array}{l}\sqrt{ } \\
- \\
-\end{array}$ \\
\hline $\begin{array}{c}\text { cross-section shape: } \\
\text { rounded } \\
\text { slab-like } \\
\text { pitched roof }\end{array}$ & $\begin{array}{c}(\sqrt{ }) \\
\sqrt{ } \\
-\end{array}$ & $\begin{array}{c}\sqrt{ } \\
- \\
(\sqrt{ })\end{array}$ & $\begin{array}{l}\sqrt{ } \\
- \\
-\end{array}$ & $\begin{array}{c}(\sqrt{ }) \\
- \\
\sqrt{ }\end{array}$ \\
\hline $\begin{array}{c}\text { fiber shape: } \\
\text { straight } \\
\text { bent } \\
\text { kinks } \\
\text { branches } \\
\text { general description: }\end{array}$ & $\begin{array}{l}- \\
- \\
\sqrt{ } \\
\sqrt{ } \\
-\end{array}$ & $\begin{array}{c}\sqrt{ } \\
- \\
- \\
- \\
\text { lego-fibers, } \\
\text { carnivore-teeth }\end{array}$ & $\begin{array}{c}- \\
\sqrt{ } \\
- \\
- \\
\text { worms }\end{array}$ & $\begin{array}{c}\sqrt{ } \\
- \\
\sqrt{ } \\
\sqrt{ } \\
\text { fibers, earthworm } \\
\text { with combat-crown }\end{array}$ \\
\hline fibers taper off & - & - & $\sqrt{ }$ & $\sqrt{ }$ \\
\hline fiber length & short & medium & medium & very long \\
\hline
\end{tabular}

Tab. 8.4: Tailoring nanofibers: The influence of molecular building blocks's substituents - mono functionalised $p$-quaterphenylenes. 


\section{Conclusions and Outlook}

starting from a functionalised molecular building block and accordingly will guide the synthesis, which serves as a key step.

Since only an overview is given of the variety of nanoaggregates and their tailor-made properties, it is self-evident to organise further (quantitative) examinations on the most promising molecular building blocks and their nanostructures. This will be the next step towards optoelectronic device development based on nanofibers, after the first step of fiber formation has been demonstrated in this thesis.

For this thesis only optical and morphologically properties have been born in mind. For future research also improvement of electrical properties will be considered, while still conserving the concept of crystalline nanofibers. Promising canditates are the phenyl thiophene co-oligomers. Therefore a first outline of the acceptability of these co-oligomers for nanofiber growth on muscovite mica is given in the following chapter. Experiments concerning e.g. electrical conductivity and electroluminescence will be part of future investigations. Apart from this the co-oligomers show tunable green fluorescence, which widens the spectral range of the nanofiber's tailorable luminescence into the green. 


\section{Outlook: Phenyl-thiophene co-oligomers [10]}

Another class of important rod-like molecules for photonic and optoelectronic applications are thiophene oligomers [27, 33]. These kind of organic semiconductors have been synthesised for the first time more than 60 years ago [226, 227]. Later research showed that they are promising candidates for electronic devices, such as organic field effect transistors (OFET) 228] and organic light emitting diodes (OLEDs) [229, 230]. OFETs based on $\alpha$-sexithiophene or derivatives present high field-effect mobility for such organic based devices. It is challenging to control emission characteristics of conjugated oligomers. Thiophene oligomers have been studied as model compounds to interprete charge-transfer mechanisms since these heterocyclic materials exhibit both interesting electrical and optical properties and possess thermal and chemical stability [231]. In the previous chapters the suitability of phenylene oligomers in the face of application in optoelectronic and photonic devices has been elucidated. Hybridising and blending of materials are common attempts to improve desired properties, which can be done on a molecular level. Thiophene/phenylene co-oligomers, i.e. hybridised oligomers containing thiophene- and phenyl-moieties, have been developed and synthesised independently by Hotta et al. 232] and Samulski et al. [233] in view of applications in photonic or optoelectronic devices. Further co-oligomers and additionally perfluorophenyl-thiophenes have been synthesised by Facchetti and Marks et al. more recently [81]. One of the major advantages of this novel class of organic semiconductors is that the $\pi$-conjugation length, and hence the emission colour can be tuned as desired by changing the total number of thiophene- and phenyl-rings and their mutual arrangement within the molecule. Various molecular shapes occur, namely straight, bent, or zigzag. Guided by this simple molecular design principle, these oligomers are predestinated to explore structure-property relationship for organic semiconductors. These phenyl-thiophene co-oligomers have attracted attention due to their potential as active components in OLEDs, organic diode lasers, and p-type semiconductors in OFETs [80, 234-243]. Noteworthy features are electroluminescence [237, and emission gain narrowing like spectrally narrowed emission (SNE) [244-246], and amplified spontaneous emission (ASE) caused by self-waveguide effects [247 249], and even mirrorless lasing [250, 251]. 


\subsection{Synthetic Approach}

Modification of para-phenylene oligomers with thiophene moieties leads to a new class of light emitting organic semiconductors: either phenyl-endcapped oligothiophenes, thienylendcapped oligophenylenes, or alternating oligomers with a phenyl- as well as a thienylendcap, respectively. However, quite a large number of phenyl-thiophene co-oligomers is accessible by changing the overall number of thiophene- and phenyl-rings as well as their mutual arrangement within the molecule. Several of these oligomers have already been synthesised via Grignard, Stille coupling or Suzuki cross-coupling reactions [81, 223, 252256]. The properties of these co-oligomers can be altered not only by varying the number of phenyl and thiophene units but also by changing the sequence. Starting from blue light emitting $p$-hexaphenylene $(\mathrm{p} 6 \mathrm{P})$ the introduction of thiophene units will cause a redshift of the fluorescence since the HOMO - LUMO gap will be decreased [240]. In addition a pronounced redshift is observed by translating the thiophene unit from the terminal position to the center of the molecule [233, 257]. This redshift is explained by a reduced torsion angle between thiophene and adjacent phenyl rings, which results in a more coplanar arrangement with improved conjugation and thus leads to a red shifted emission. This is true for considerations in solution but the trend is retained for the solid state. The thermal stability of phenyl endcapped thiophenes should be increased in comparison to oligothiophenes and thienyl endcapped $p$-phenylenes. A terminal thiophene unit is susceptable to heat induced reations such as polymerisation or decomposition because of the unsubstituted alpha-position.
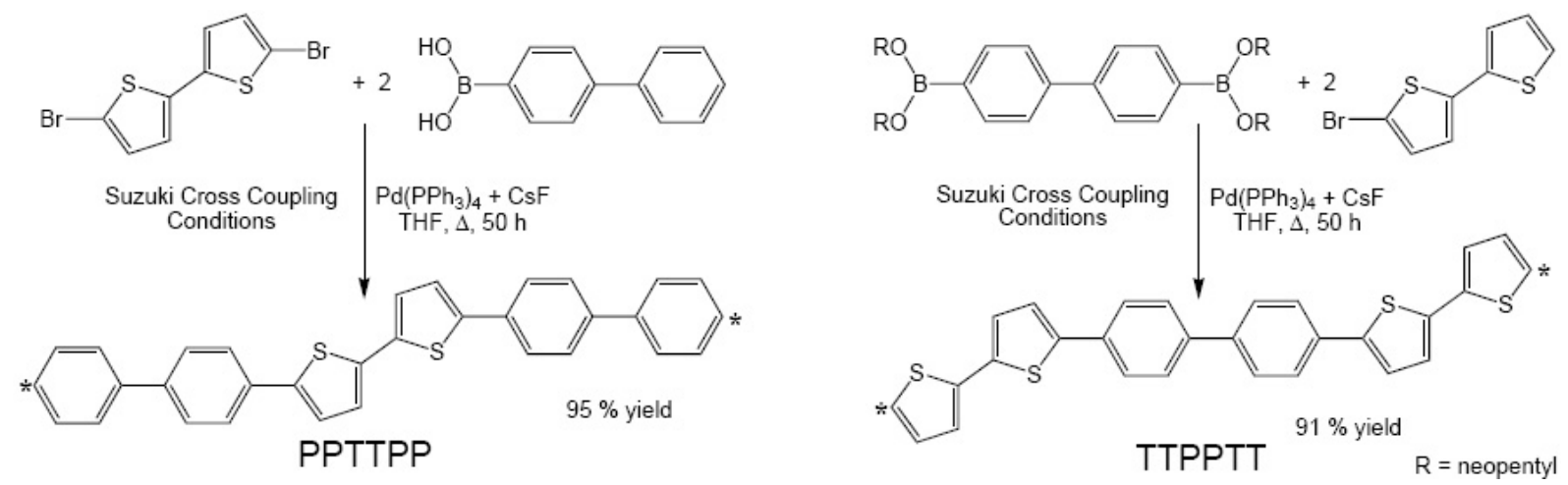

Fig. 9.1: Synthesis of phenyl-thiophene co-oligomers via Suzuki cross-coupling reaction. The line connecting the carbon atoms marked with asterisks represents the long molecular axis.

Here it is reported on two different phenyl thiophene co-oligomers: the phenyl endcapped 5,5'-Di-4-biphenyl-2,2'-bithiophene (PPTTPP) and the thienyl endcapped 4,4'Di-2,2'-bithienyl-biphenyl (TTPPTT). The synthesis of these and related oligomers has been published before [81, 223] but this novel approach by Suzuki-cross-coupling reaction 
(Fig 9.1) provides higher yields:

\begin{tabular}{c|cc}
\hline & TTPPTT & PPTTPP \\
\hline yield [\%] & 95 vs. 77 (Facchetti, Marks et al. [81]) & 91 vs. 57 (Hotta et al. [223]) \\
\hline
\end{tabular}

Facchetti and Marks et al. utilised a two-fold Stille coupling starting from a 2,2'stannyl-bithiophene and 4,4'dibromo-biphenyl and tetrakis(triphenylphosphino)palladium as catalyst in dry dimethyl formamide to achieve TTPPTT. Hotta et al. synthesised PPTTPP by means of a two-fold Suzuki cross coupling reaction of the appropriate $\alpha$-iodo thiophene and $p$-phenyl boronic acid using again tetrakis(triphenylphosphino)palladium as catalyst together with aqueous sodium carbonate in chlorobenzene.

In the present thesis the two symmetric oligomers have been synthesised in a twofold Suzuki cross-coupling reaction from commercially available $\alpha$-bromo thiophenes and para-phenyl boronic acids or esters, using $5 \mathrm{~mol} \%$ tetrakis(triphenylphosphino)palladium as catalyst together with caesium fluoride as base in dry tetrahydrofurane. The desired products have been obtained in yield more than $90 \%$ each after refluxing for $50 \mathrm{~h}$. The final products precipitated from the reaction mixture and have been washed with water and organic solvents repeatedly for purification. By outgassing in vacuo residual organic solvents are removed to give the desired compounds in high purity.

\subsection{Growth}

\subsubsection{PРTTPP}

Deposition in high vacuum of the biphenyl-endcapped thiophene PPTTPP onto a freshly cleaved muscovite mica substrate at elevated substrate temperatures leads to green fluorescent fiberlike nanostrucures forming a rhombic pattern.

The nanostructures form two domains on a single sample, within each domain two fiber orientations occur forming rhombi with the acute angle being $80^{\circ} \pm 3^{\circ}$. The length of the fibers is up to $20 \mu \mathrm{m}$, and they possess heights and widths of few ten and few hundred nanometer, respectively. The length is limited by the number density of the fibers: after growing together of the two needle directions the increase in length is stopped. The fibers show no alignment along the mica high symmetry directions, similar to thiophene oligomers on muscovite mica [63]. The crystallographic directions have been found out by means of a Schlagfigur.

The nanostructures possess a high degree of crystallinity, emitting strongly polarised green light after excitation with unpolarised UV-light under normal incidence. The molecular arrangement determines the optical properties of the nanofibers. The oligomer has a transition dipole roughly along its long axis, i.e. the direction of the transition dipole is in good accordance with the long molecular axis, which is defined by a line connecting 

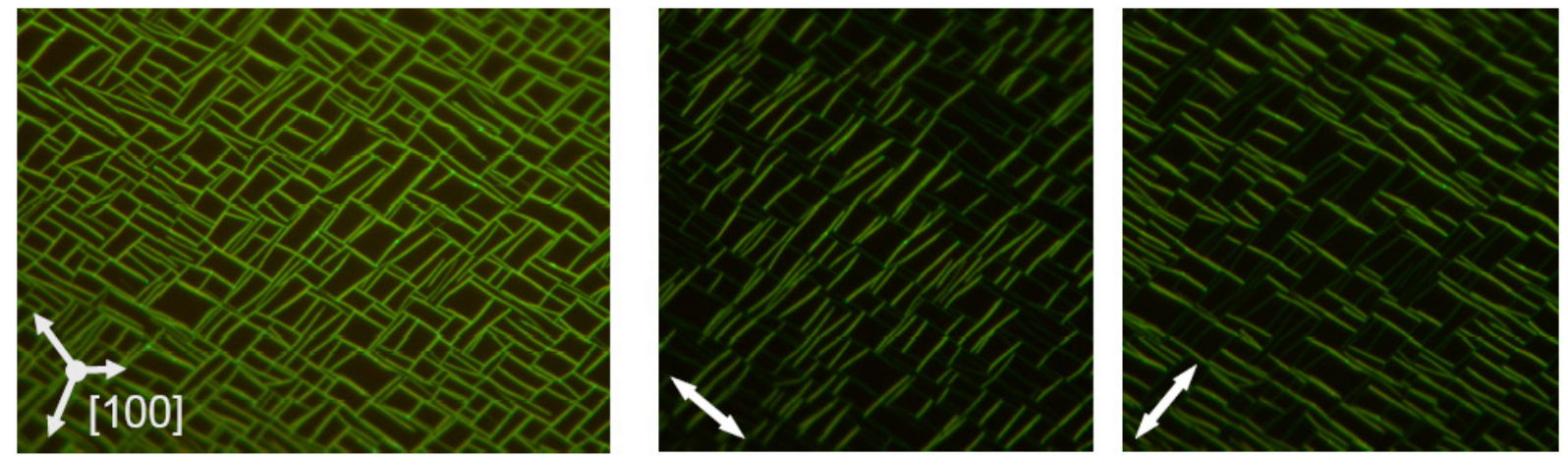

Fig. 9.2: Fluorescence microscopy images $\left(\lambda_{e x c}=365 \mathrm{~nm}, \mathrm{~T}_{S}=400 \mathrm{~K}\right)$ of PPTTPP on muscovite mica. The image $\left(113 \times 85 \mu \mathrm{m}^{2}\right)$ on the left is taken without a polariser, the images $\left(85 \times 85 \mu^{2}\right)$ in the middle and on the right are taken through a polariser to point out the two directions of polarisation (depicted by white arrows).

(a)

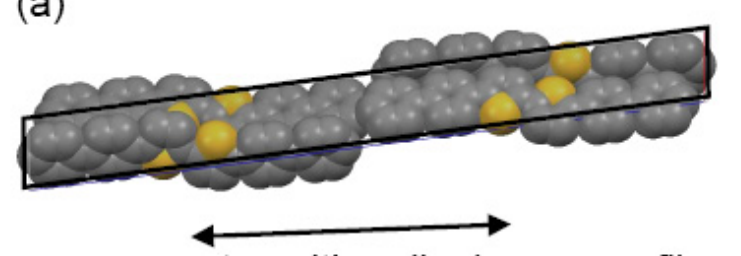

transition dipole

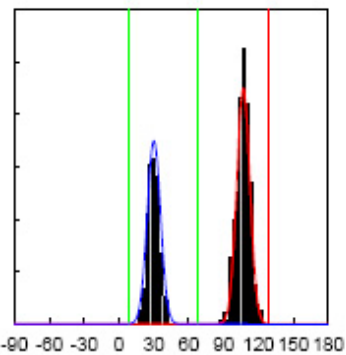

needle angle $\left[^{\circ}\right]$

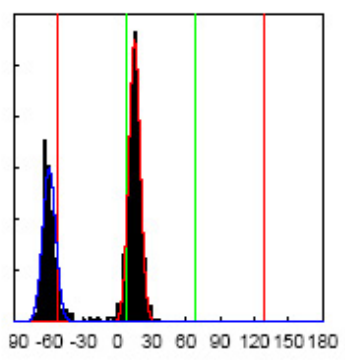

polarisation $\max \left[{ }^{\circ}\right]$

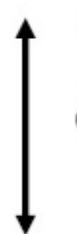

(b)

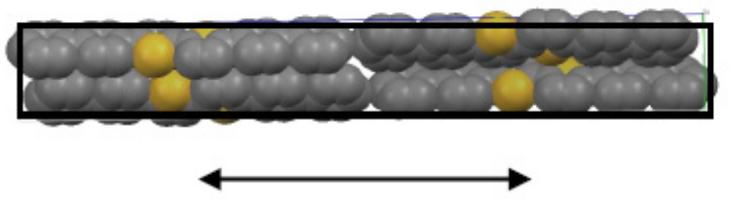

transition dipole

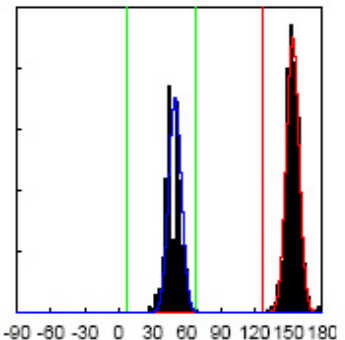

needle angle $\left[{ }^{\circ}\right]$

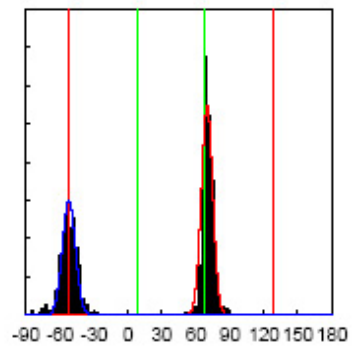

polarisation $\max \left[{ }^{\circ}\right]$

Fig. 9.3: Possible orientations of the PPTTPP oligomers within a fiber: (a) view on $a c$-plane and (b) view on $b c$-plane of the unit cell, which are considered to face the substrate. The transition dipoles are along the long molecular axis, which is defined by a line connecting the carbon atoms at the 1-,1'-positions. Two fiber directions and each with its own polarisation direction exist within one domain (cf. graphs: number of needles vs. needle angle and polarisation maxium, respectively; green lines indicate muscovite $\left\langle\begin{array}{lll}1 & 1 & 0\end{array}\right)$ and red lines muscovite $\left[\begin{array}{lll}1 & 0 & 0\end{array}\right]$ ). Gaussian fits show that for some domains light is only polarised almost parallel to muscovite high symmetry directions (a), but for other domains light is exactly polarised parallel to muscovite high symmetry directions (b). This could reflect two different crystal faces being parallel to the substrate. 
the carbon atoms at the 1-,1'-positions [258]. The emitted fluorescence light is always polarised almost perpendicular to the long fiber axis. This indicates that the oligomers are lying with their long axes almost perpendicular to the long fiber axes. According to this in a simple conclusion the fibers can orientate with two different crystal faces parallel to the substrate: either the $a c$ plane ( $\left(\begin{array}{lll}0 & 1 & 0\end{array}\right)$ face) or $b c$ plane $\left(\left(\begin{array}{lll}1 & 0 & 0\end{array}\right)\right.$ face) of the unit cell can face the substrate (Fig.9.3).

Each fiber direction has its own polarisation direction, i.e. two polarisation directions exist within one domain. The light is polarised almost parallel to muscovite $\left[\begin{array}{lll}1 & 0 & 0\end{array}\right]$ and $\langle 110\rangle$ respectively (Fig 9.3). The plane of polarisation is never along the muscovite $\langle 110\rangle$ direction which exhibits grooves, similar to the case of thiophene oligomers [63]. Actually two slightly different domains exist: For some domains light is only polarised almost parallel to muscovite high symmetry directions, but for other domains light is exactly polarised parallel to muscovite high symmetry directions. This could reflect two different crystal faces being parallel to the substrate. It has to be clarified in the future if this is a true phenomenon or if it is due to rotation of the polarisation within the microscope's mirror and lens setup. However, the molecules orientate with their long axes (almost) parallel to the electric fields of the mica substrate (Fig 9.4). That way, the two fiber directions of the phenyl-thiophenes as well as the single fiber direction of the (functionalised) $p$-phenylenes can be explained by the same growth model: The driving force for the alignment of the molecules is the maximised interaction between the electric surface fields of the muscovite mica substrate and the induced dipole along the oligomer's long axis.

Similar fiberlike structures have been obtained by Yanagi et al. [80, 237, 241] after vapour deposition of PPTTPP onto a cleaved ionic $\mathrm{KCl}(100)$ surface at elevated substrate temperatures. The green fluorescent fiberlike crystals epitaxially grow along the orthogonal $\langle 110\rangle$ directions of the $\mathrm{KCl}$ substrate. That way the fibers form rectangular patterns. Within the fibers the oligomers lie parallel to the surface and align perpendicular to the long fiber axis as demonstrated by X-ray and electron diffraction patterns. This confirms the polarisation measurements since the transition dipole of the molecules is parallel to the molecular axis: The fluorescence light after UV-excitation is polarised perpendicular to the long fiber axis. XRD-patterns suggest that the crystalline fibers have either their $(100)$ or $(010)$ faces, i.e. $b c$-plane or $a c$-plane in contact with the $\mathrm{KCl}$ surface [241] as assumed similar for the case of PPTTPP on muscovite mica.

PPTTPP has been deposited on a muscovite mica substrate for different substrate temperatures, namely a temperature range from room temperature to $460 \mathrm{~K}$ is covered. The morphology of the nanostuctures is rather independent from the substrate temperature: for the whole temperature range a rhombic pattern is formed. For low substrate temperatures the side lengths of the rhombi is clearly decreased. For substrate tempera- 

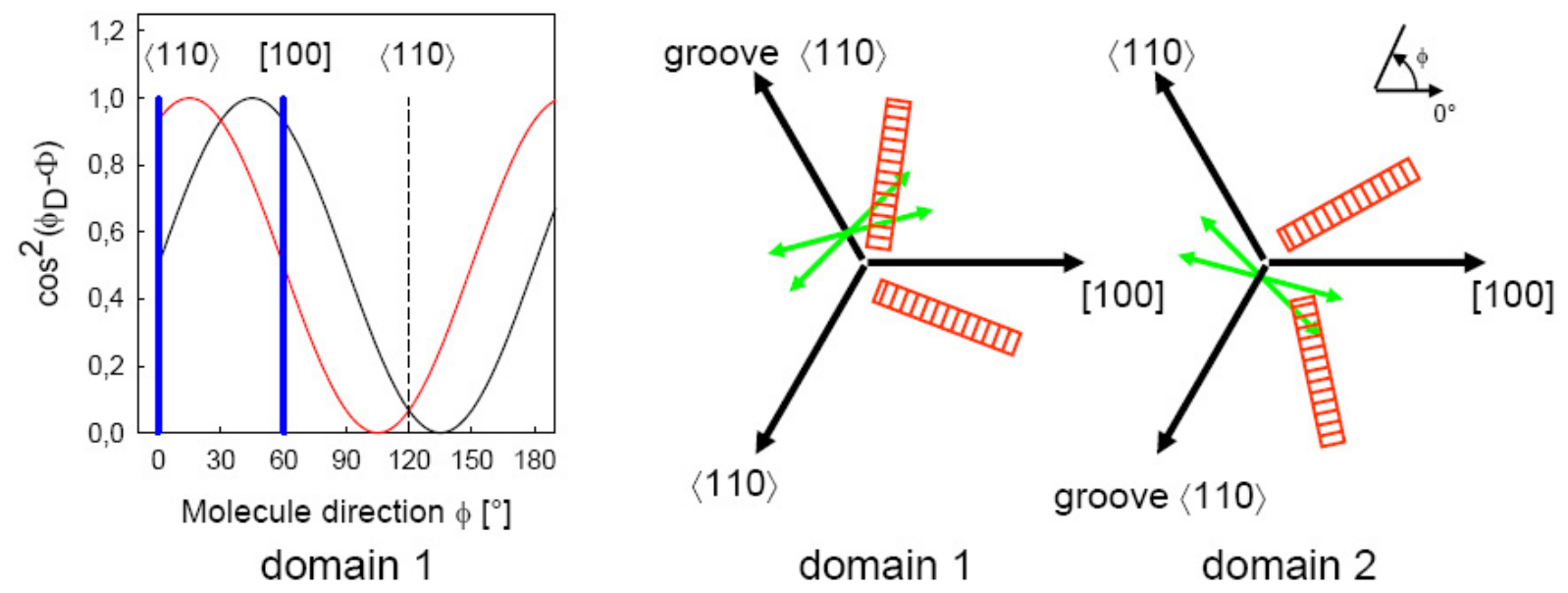

Fig. 9.4: The graph (left) shows relative term of interaction energy between surface electric fields and induced dipolemoment along the long molecular axis depending on the fiber orientation for a single rotational domain (domain 1). The orientations of PPTTPP fibers within two different muscovite mica domains are sketched, together with the oligomers' orientation within the fiber. Muscovite high symmetry directions are depicted by black arrows, assumed suface dipol directions by short green arrows.

tures above $440 \mathrm{~K}$ no fibers can be observed, only some fluorescent islands remain on the surface, similar to e.g. p6P.

Fig. 9.5 shows atomic force microscopy images of PPTTPP on muscovite mica for different substrate temperatures and nominal film thicknesses. For increased film thicknesses small clusters with a height of about $10 \mathrm{~nm}$ on top of the fibers are visible (Fig.9.5(c)), similar to thiophenes [63]. For moderate film thicknessess the fibers possess an even morphology. In between the fibers small clusters are located, which are believed to be needle-precursors similar to e.g. p6P [66 68]. Also expanded islands exhibiting flat terraces with $2.5 \mathrm{~nm}$ or $5 \mathrm{~nm}$ high steps indicating upright standing molecules on the surface (Fig.9.5(a)) are visible between the fibers. Sometimes on top of these island tall aggregates with heights up to $200 \mathrm{~nm}$ grow because of the decrease in substrate surface energy [259], which means that they are more than double as high compared to the fibers. The tall aggregates are non-fluorescent just like the islands. This lack of fluorescence is a clear hint that islands and tall aggregates are built up of upright standing oligomers.

\subsection{2 ТТРРТТ}

Deposition in high vacuum of the bithienyl-endcapped biphenyl TTPPTT onto a freshly cleaved muscovite mica substrate leads to green fluorescent fiberlike nanostrucures forming a pattern which reminds one of fir-needles.

TTPPTT has been deposited on a muscovite mica substrate for different substrate tem- 

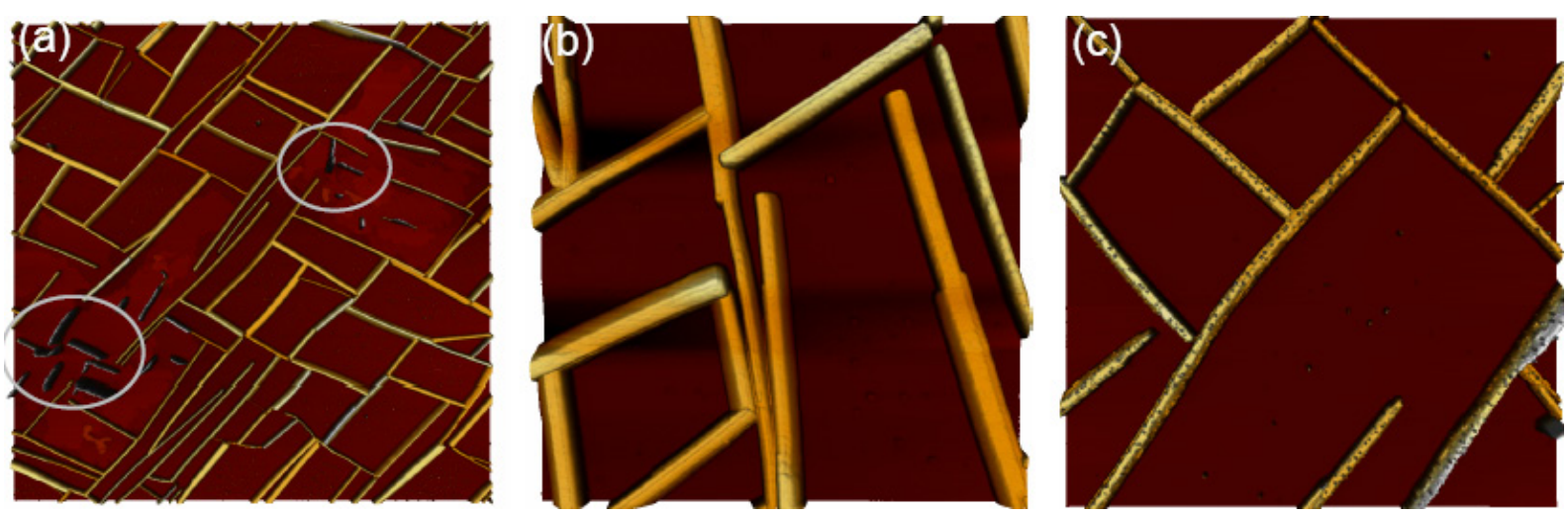

Fig. 9.5: AFM-images of PPTTPP on muscovite mica for different substrate temperatures and nominal film thicknesses: (a) $\mathrm{T}_{S}=385 \mathrm{~K}, 5 \mathrm{~nm}, 20 \times 20 \mu \mathrm{m}^{2}$; (b) $\mathrm{T}_{S}=$ $350 \mathrm{~K}, 5 \mathrm{~nm}, 5 \times 5 \mu \mathrm{m}^{2}$; (c) $\mathrm{T}_{S}=400 \mathrm{~K}, 15 \mathrm{~nm}, 10 \times 10 \mu \mathrm{m}^{2}$ : height scales $76 \mathrm{~nm}$. Tall non-fluorescent aggregates growing on non-fluorescent flat islands are marked by the circles.
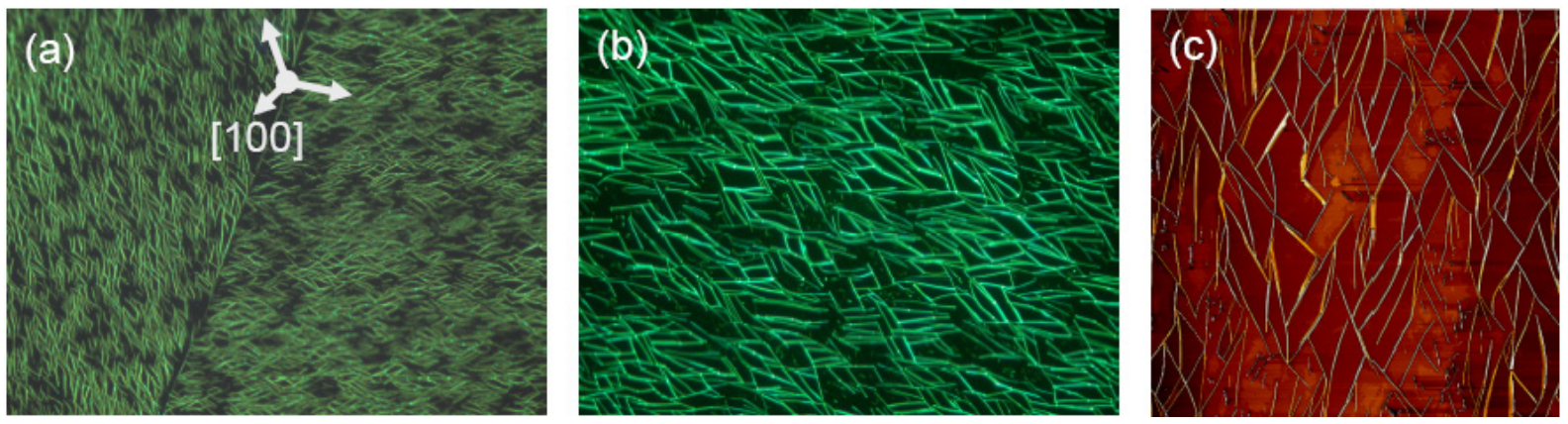

Fig. 9.6: Fluorescence microscopy images $\left(\lambda_{e x c}=365 \mathrm{~nm}\right)$ of TTPPTT on muscovite mica (a) $675 \times 500 \mu \mathrm{m}^{2}, \mathrm{~T}_{S}=400 \mathrm{~K}$, (b) $250 \times 188 \mu \mathrm{m}^{2}, \mathrm{~T}_{S}=420 \mathrm{~K}$, and an AFM-image (c) $80 \times 80 \mu \mathrm{m}^{2}, \mathrm{~T}_{S}=420 \mathrm{~K}$, height scale $45 \mathrm{~nm}$.

peratures, namely a temperature range from room temperature to $450 \mathrm{~K}$ is covered. The morphology of the nanostuctures is rather independent from the substrate temperature: for the whole temperature range fir-needle like structures are formed. For low substrate temperatures the lengths of the needles is clearly decreasd. For substrate temperatures above $440 \mathrm{~K}$ no fibers can be observed, only some fluorescent clusters remain on the surface.

The fir-needles form two domains on a single sample, exhibiting needles, which seem rather unorganisedly distributed around muscovite $\langle 110\rangle$ directions. The needles show no alignment exactly along the mica high symmetry directions similar to thiophene oligomers on muscovite mica [63]. The length of the fibers obtains several ten micrometer, and they possess heights and widths of few ten and few hundred nanometer, respectively.

As found to be typical for thiophenes, this bithienyl-endcapped oligomer crystallises 

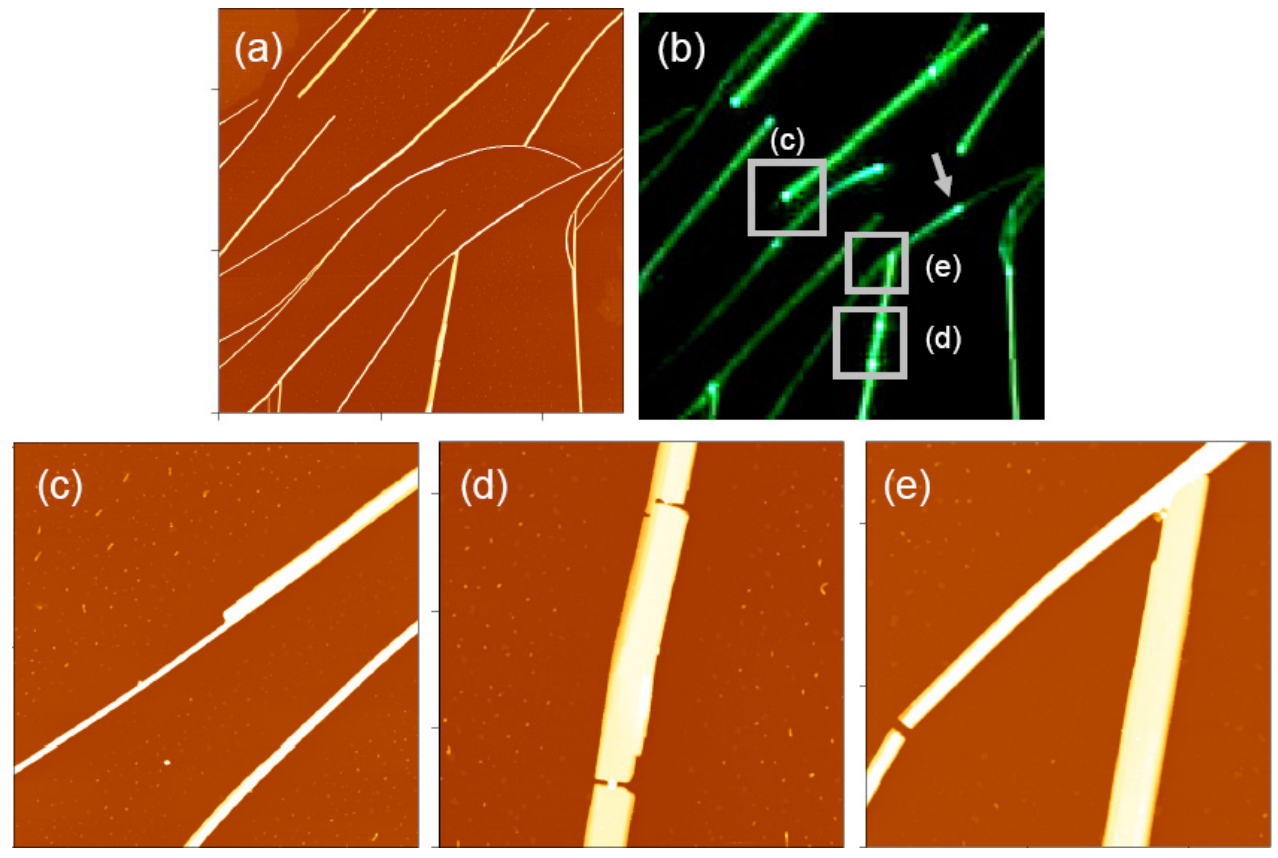

Fig. 9.7: AFM images of TTPPTT on muscovite mica (a) $50 \times 50 \mu \mathrm{m}^{2}$, (c) $10 \times 10 \mu \mathrm{m}^{2}$, (d) $7 \times 7 \mu \mathrm{m}^{2}$, and (e) $5 \times 5 \mu \mathrm{m}^{2}$, all height scales are $70 \mathrm{~nm}$. Fluorescence microscopy image (b) shows the same area as (a). From [10].

in large areas of upright molecules in between the needles, too. This areas can be seen

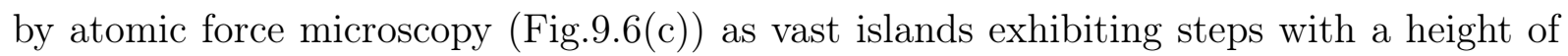
$2.7 \mathrm{~nm}$ or $3.0 \mathrm{~nm}$. These islands are non-fluorescent because they are formed of upright standing molecules on the surface. In the literature it has been discussed for $\alpha \mathrm{T} 6$ [164], that two different types of island exist: Either islands consisting of a simple layer of upright standing molecules (refers to stepheight of $2.7 \mathrm{~nm}$ ) or islands consisting of a layer of lying molecules with a layer of upright standing molecules on top (refers to stepheight of 3.0 $\mathrm{nm})$. Sometimes tall aggregates with heights up to $200 \mathrm{~nm}$ grow on top of those islands, Fig 9.6(c), similar to PPTTPP, Fig.9.5(a). These non-fluorescecent tall aggregates of upright standing oligomers appear as black areas in fluorescecence microscopy images. The fluorescent fir needle are located in between the detritic islands.

Often fluorescence microscopy images show bright spots within the needles. These glowing spots usually occur at fiber ends or at breaks within a single fiber, which can be seen by high resolution AFM images (Fig 9.7(c),(d)) and their corresponding fluorescence microscopy image. Phenyl-thiophenes are known to act as waveguides from a certain width [46, 57], therefore these bright spots can by attributed to waveguided light, which is scatterd at breaks and endings. However, not all breaks appear as bright spots (Fig 9.7(e)). For $500 \mathrm{~nm}$ light, the maximum of the emission spectrum of TTPPTT needles, the condition for waveguiding is a minimum width of the guiding fiber of about $290 \mathrm{~nm}$ [47. The broken needle in (d) has a width of $780 \mathrm{~nm}$, wheras the one in (e) is only $250 \mathrm{~nm}$ 

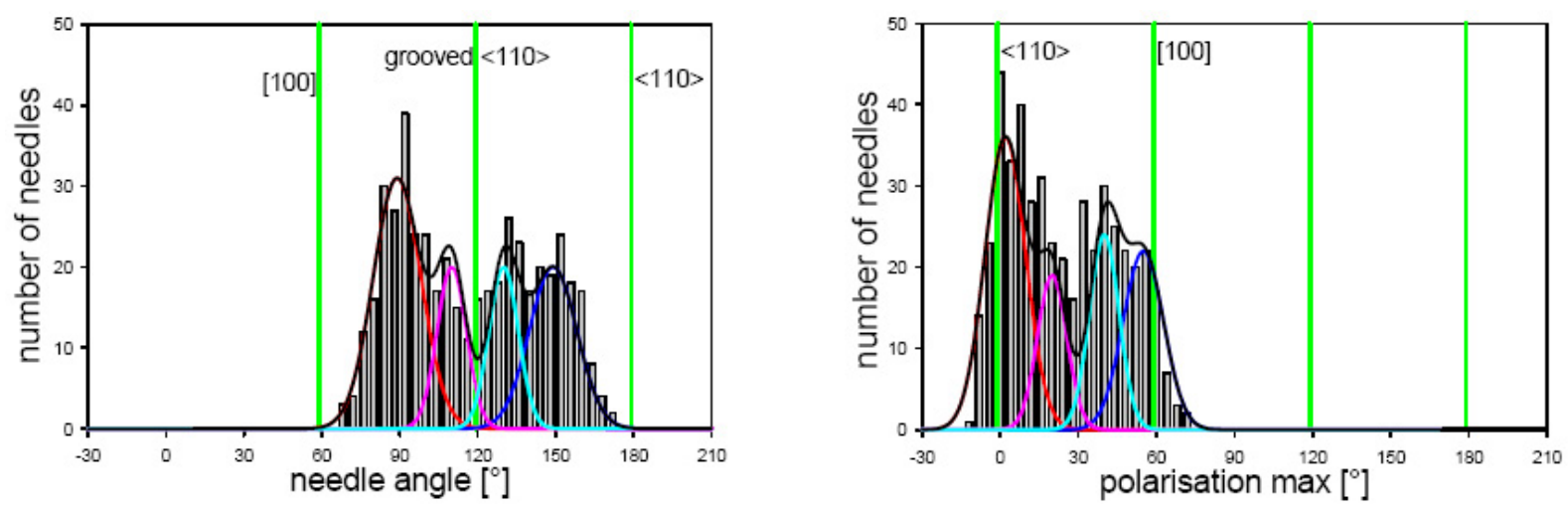

Fig. 9.8: The graphs show the needle distribution (left) and the corresponding polarisation direction (right) with respect to the high symmetry directions for one domain of TTPPTT on muscovite mica. Gaussian fits reveal four main needle directions each with its own corresponding direction for the emitted polarised fluorescence light.

wide, thus prohibiting waveguiding for the latter one. Following this narrow fiber to the upper right it is notably becoming wider, reaching $330 \mathrm{~nm}$. At this point waveguiding is possible, leading to the bright spot marked by an arrow in Fig 9.7(b).

Since the emitted green fluorescence light is strongly polarised, the fir needles possess a high degree of crystallinity. Because of the herringbone packing of the molecules in the bulk (cf. Tab 9.1) and the lowest lying transition dipole moment being in good approximation along the long molecular axis, which is defined to be along a line connecting the carbon atoms at the 2-2'-positions, the molecule's orientation on the surface can be deduced from analysing the polarised emission of fluorescecne light after unpolarised excitation with UV light. A statistical analysis of fluorescence and AFM images (Fig 9.8) shows that the broad distribution of fir-needles around muscovite $\langle 110\rangle$ is more organised than it seems at first glance. Four main needle directions exist within a single domain, which are distributed around grooved muscovite $\langle 110\rangle \pm 10^{\circ}$ and $\pm 30^{\circ}$, respectively (experimental error $\pm 5^{\circ}$ ). Each needle direction has its own polarisation maximum, which is always exactly perpendicular to the long needle axis. This results in polarisation direc-

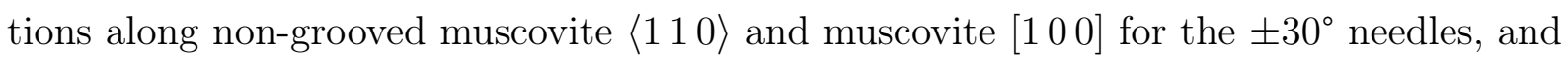
polarisation directions along the surface dipoles for the $\pm 10^{\circ}$ needles. The grooved $\langle 110\rangle$ direction is not selected as molecule direction because of the unfavourable orientation with respect to the surface electric fields, just like for (functionalised) $p$-phenylenes and thiophenes. This leads to a growth model as sketched in Fig 9.9. That way, the driving forces for the alignment of the phenyle thiophene co-oligomers are on the one hand maximised interaction between the muscovite mica's surface dipoles and the induced dipole along the oligomer's long axis and on the other hand alignment along high symmetry directions of the substrate, i.e. epitaxy. 

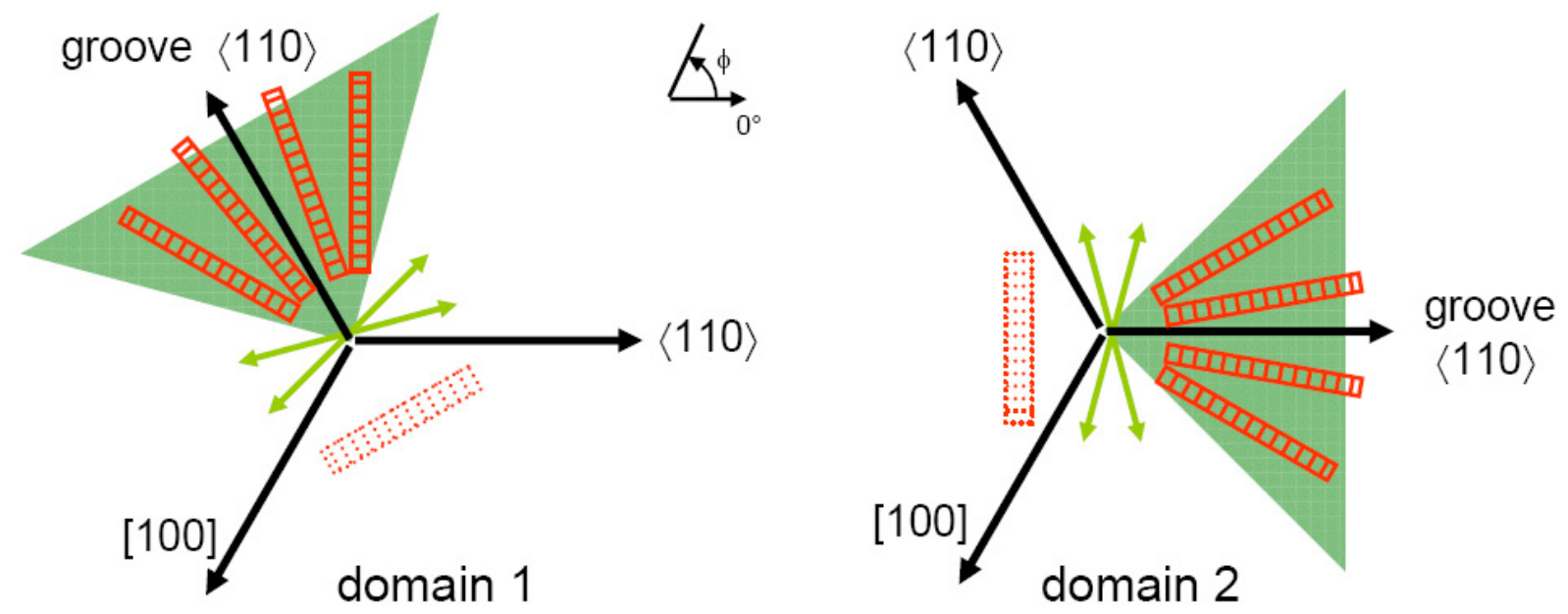

Fig. 9.9: Growth model of TTPPTT on muscovite mica for two different domains. Green triangle shows the distribution range of needles, which are pictured by the red rods for the four main needle orientations along grooved muscovite $\langle 110\rangle \pm 30^{\circ}$ and $\pm 10^{\circ}$. Green arrows depict the surface dipoles. Oligomers orientate along non-grooved muscovite $\langle 110\rangle$ and muscovite $\left[\begin{array}{ll}1 & 0\end{array}\right]$, respectively, for the $\pm 30^{\circ}$ orientation, and along the surface dipoles for the $\pm 10^{\circ}$ orientation. The dotted red rods illustrate the not realised needle direction with the oligomers oriented along grooved muscovite $\langle 110\rangle$.

\subsection{Linear Optics}

The polarisation properties of the emitted fluorescence light depend on the molecular orientation within the nanoaggregates, which is different for the two oligomers and have been discussed in the previous section.

Fluorescence spectra have been recorded after continous wave UV excitation of the nanoaggregates on the muscovite mica substrate at $325 \mathrm{~nm}$. The spectra, both within the green, can be seen in Fig 9.10. They show well resolved vibronic structures. The finetuning of the emission colour depends on the total number of of phenyl- and thiophenerings and on their mutual arrangement within the molecule. Most probable (0-1) transitions for nanoaggragates of various compounds are given in the following table for comparision:

\begin{tabular}{c|cccccc}
\hline molecule & $\mathrm{p} 4 \mathrm{P}$ & $\mathrm{p} 6 \mathrm{P}$ & $\mathrm{TTPPTT}$ & $\alpha 4 \mathrm{~T}[63]$ & PPTTPP & $\alpha 6 \mathrm{~T}[63]$ \\
\hline (0-1) transition $[\mathrm{nm}]$ & 386 & 422 & 500 & 512 & 523 & 590 \\
\hline
\end{tabular}

In general, thiophenes fluoresce at higher wavelength, i.e. have a more red-shifted fluorescence, compared to phenylenes possessing the same number of rings within the oligomer because of the electron-rich sulfur atom included in the thiophene ring. The longer the oligomer becomes, i.e. the higher the total number of rings, the more red-shifted the 


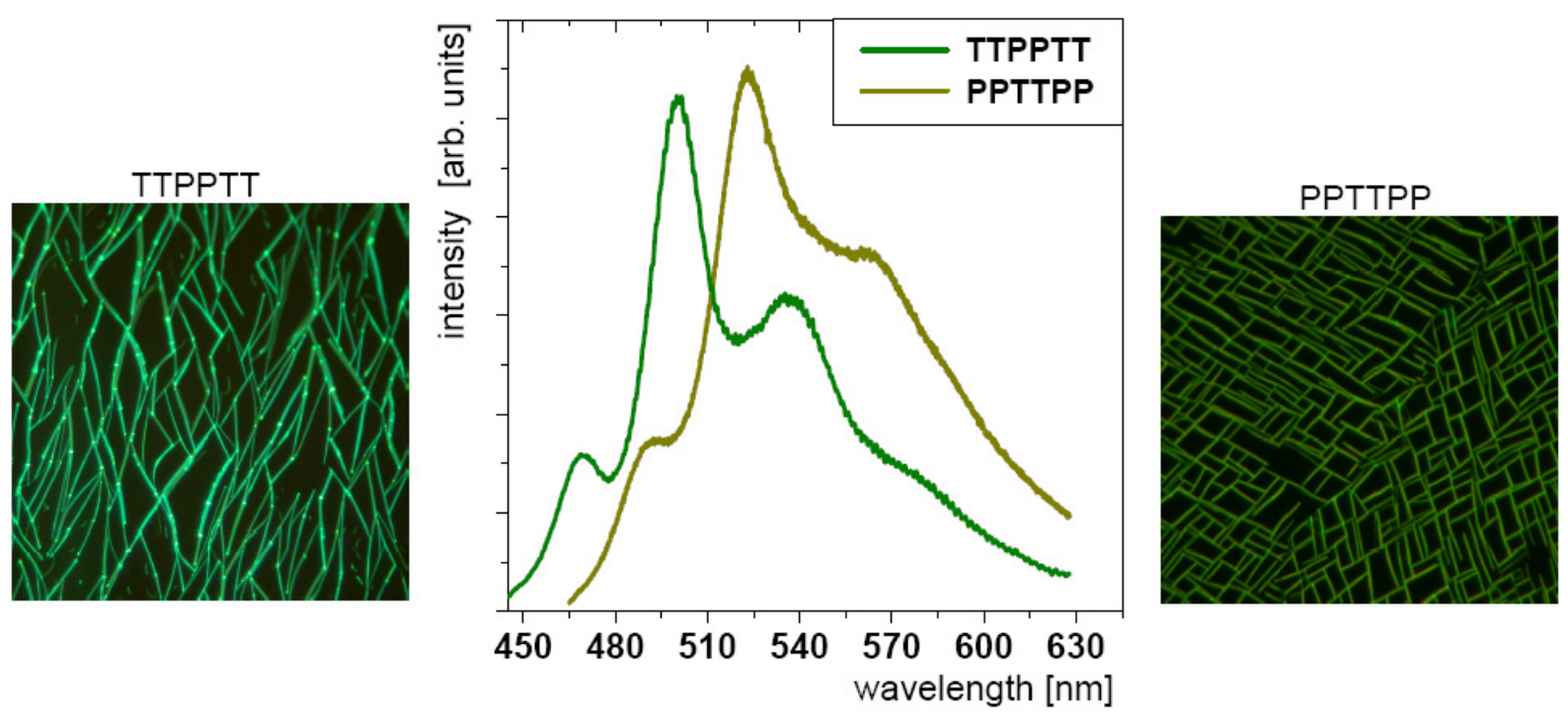

Fig. 9.10: Fluorescence spectra have been obtained after $\mathrm{cw}$ excitation at $325 \mathrm{~nm}$ from the phenyl-thiophenes on muscovite mica. The differences within the green colour can be seen by eye: fluorescence microscopy images $\left(\lambda_{e x c}=365 \mathrm{~nm}\right)$ of TTPPTT (left, $130 \times$ $130 \mu \mathrm{m}^{2}$ ) and PPTTPP (right, $85 \times 85 \mu \mathrm{m}^{2}$ ).

fluorescence. One would expect that the fluorescence of TTPPTT is more red-shifted due to more electron-rich thiophene-moeties incorporated in the oligomer compared to PPTTPP. But the fluorescence of TTPPTT is even blue-shifted compared to $\alpha 4 \mathrm{~T}$, although the number of thiophene-units is the same and TTPPTT even possesses two additional phenyl-rings. This can only be explained by confering the crystal structures and in more detail by confering the conjugation of the $\pi$-electrons, which determines the colour of the fluorescence light (Fig9.11. Tab 9.1). Both phenyl-thiophenes consist of zigzag-shaped oligomers. The crystals are characterised by a molecular layered structure, in which the molecules form the well-known herringbone structure laterally spreading along the $a b$-plane. A remarkable feature is that the long molecular axis is almost perpendicular to the $a b$-plane depending on the projection plane in contrast to $\mathrm{p} 6 \mathrm{P}$ or $\alpha 6 \mathrm{~T}$ which posses large tilting angles (up to $25^{\circ}$ depending on the projection plane [63]). The long molecular axis is defined to be along a connecting line between the carbon atoms at the 1-1 '-positions for PPTTPP and at the 2-2'-position for TTPPTT, respectively. The direction of the transition dipole moment is in good accordance with the long molecular axis, which determines the polarisation properties. However, TTPPTT has a more "wavy" molecular alignment within the crystal wheras the aromatic rings exhibit increased coplanarity for PPTTPP. This causes enhanced conjugation of the $\pi$-electrons in cases of PPTTPP and thus results in a red-shifted fluorescence.

Values for the emission of the here discussed phenyl-thiophenes after UV excitation can also be found in the literature. A comparison is given for the most probable (0-1) 


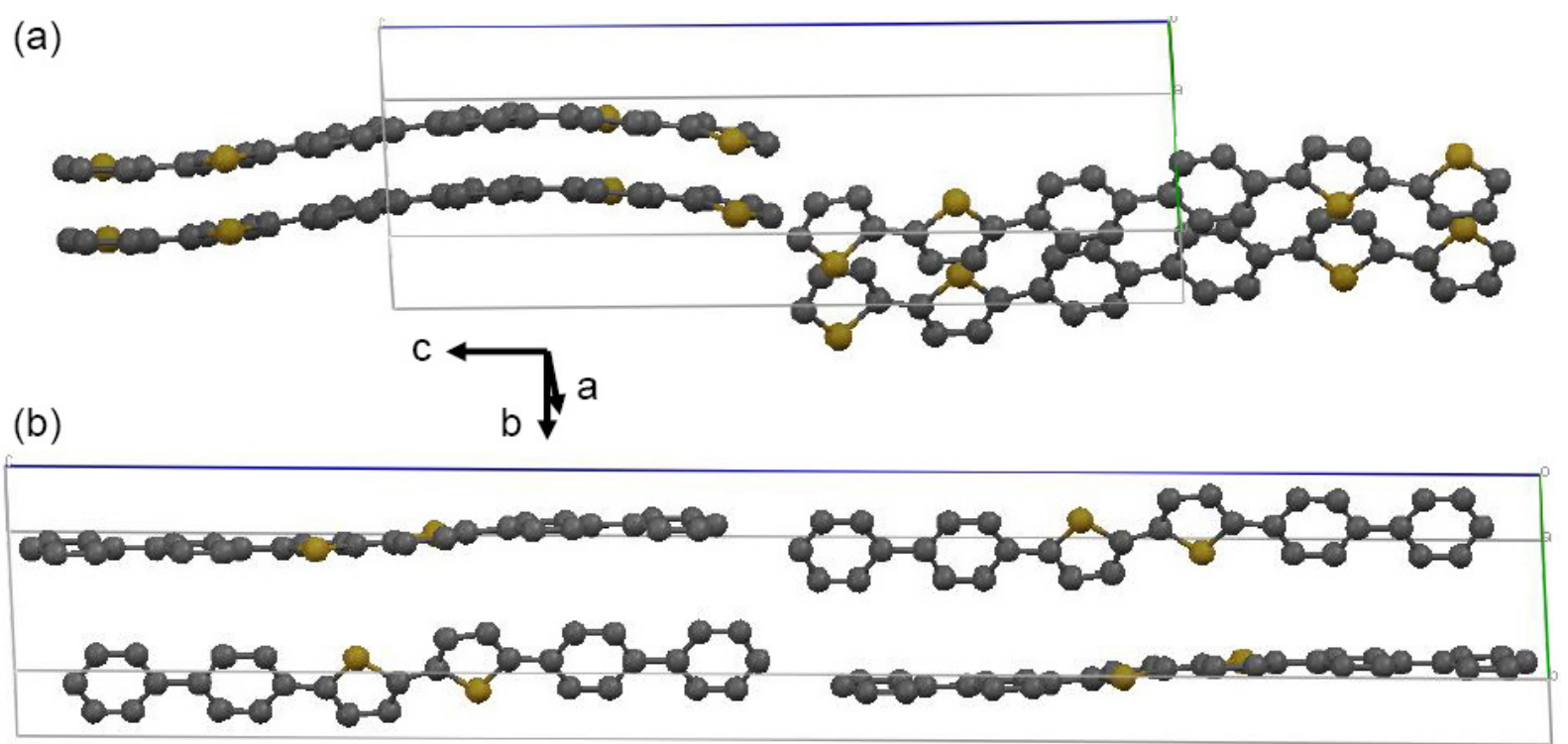

Fig. 9.11: Crystal structures of (a) TTPPTT [81] and (b) PPTTPP [258].

transition in the following table:

\begin{tabular}{c||c|c}
\hline emission wavelength [nm] for: & PPTTPP & TTPPTT \\
\hline fibers on mica (measured here) & 523 & 500 \\
film on glass & - & $500[81]$ \\
fibers on KCl & 540 [237] and 546 [242] & - \\
\hline
\end{tabular}

The here measured fluorescence peak for TTPPTT fir-needles on muscovite mica is in coincidence with the value given in the literature by Facchetti and Marks et al. for a vapour deposited film of TTPPTT on glass. The fluorescence of PPTTPP rectangles on $\mathrm{KCl}$ measured by Hotta et al. is clearly red-shifted by about $20 \mathrm{~nm}$ compared to the peak position obtained here for PPTTPP rhombi on muscovite mica. This disagreement has to be clarified in the future. However, this is not in contradiction to the discussed relationship between oligomer composition / crystal structure and tuning of the emission colour.

\subsection{Summary}

In summary, phenyl-thiophene co-oligomers form well-shaped nanostructures upon vapour deposition onto a freshly cleaved muscovite mica substrate at elevated substrate temperatures. The fiberlike nanostructures show polarised green fluorescence after unpolarised excitation with UV-light under normal incidence. The co-oligomers exhibit a much less pronounced tendency to grow sidearms compared to sexithiophene. But they show the same asymmetric behaviour with respect to the high symmetry directions of the substrate 


\begin{tabular}{c|cc}
\hline crystallographic data & TTPPTT [81] & PPTTPP [258] \\
\hline formula & $\mathrm{C}_{28} \mathrm{H}_{18} \mathrm{~S}_{4}$ & $\mathrm{C}_{32} \mathrm{H}_{22} \mathrm{~S}_{2}$ \\
formula weight & 482.66 & 470.65 \\
crystal class & monoclinic & monoclinic \\
space group & $P 2_{1}$ & $P 2_{1} / c$ \\
cell constants: & & \\
$a[\AA]$ & 5.824 & 5.708 \\
$b[\AA]$ & 7.293 & 7.603 \\
$c[\AA]$ & 25.316 & 52.869 \\
$\beta\left[\left[^{\circ}\right]\right.$ & 96.23 & 97.14 \\
$Z$ & 2 & 4 \\
herringbone angle $\left[{ }^{\circ}\right]$ & 50 & 61.0 \\
tilting angle $\left[{ }^{\circ}\right]^{\circ}$ & 1.6 & 1.2 \\
\hline
\end{tabular}

Tab. 9.1: Crystallographic data for TTPPTT and PPTTPP.

as the thiophene with missing alignment along grooved muscovite $\langle 110\rangle$ direction. However, the two growth directions of PPTTPP fibers as well as the single fiber direction of the (functionalised) $p$-phenylenes can be explained by a combination of growth epitaxy and dipole assisted alignment: The driving force for the alignment of the molecules is maximised interaction between the electric surface fields of the muscovite mica substrate and the induced dipole along the oligomer's long axis. An additional epitaxial driving force for the TTPPTT oligomers is the alignment along the non-grooved muscovite high symmetry directions. Nevertheless TTPPTT shows a more thiophene-like behaviour considering the large islands of upright standing molecules on the surface. PPTTPP behaves more similarly to $p$-phenylenes in forming mainly fiber-like structures and clusters built up from lying molecules on the surface. The optical properties, namely the fluorescence colours, are not simply given by total number and sequence of phenyl- and thiophene-units. Also the co-planarity of the aromatic rings within the nano-crystals plays an important role. However, since the fluorescence colour is within the green, this widens the spectral range of the nanofiber's tunable luminescence into the green. 
9 Outlook: Phenyl-thiophene co-oligomers [10] 


\section{Bibliography}

[1] M. Schiek, K. Al-Shamery, and A. Lützen. Synthesis of symmetrically and nonsymmetrically para-functionalised para-quaterphenylenes. Synthesis, 4, 613-621, 2007.

[2] Ivonne Wallmann. Synthese von monofunktionalisierten para-Tetraphenylenen und Thiophenphenylenen. Diploma Thesis, University of Oldenburg, Dez., 2006.

[3] M. Schiek, A. Lützen, R. Koch, K. Al-Shamery, F. Balzer, R. Frese, and H.-G. Rubahn. Nanofibers from functionalized para-phenylene molecules. Appl. Phys. Lett., 86, 153107-9, 2005.

[4] M. Schiek, A. Lützen, K. Al-Shamery, F. Balzer, and H.-G. Rubahn. Nanofibers from methoxy functionalized para-phenylene molecules. Surf. Sci., 600, 4030-4033, 2006.

[5] M. Schiek, A. Lützen, K. Al-Shamery, F. Balzer, and H.-G. Rubahn. Organic nanofibers from chloride functionalized para-quaterphenylenes. Cryst. Growth and Design, 7, 229-233, 2007.

[6] M. Schiek, J. Brewer, F. Balzer, A. Lützen, K. Al-Shamery, and H.-G. Rubahn. Tailored nanoaggregates from functionalized organic molecules. Proc. SPIE, 6475, 2007.

[7] C. Maibohm, J. Brewer, H. Sturm, F. Balzer, and H-G. Rubahn. Bleaching and coating of organic nanofibers. J. Appl. Phys., 100, 0543041-4, 2006.

[8] K. Thilsing-Hansen, M.T. Neves-Petersen, S.B. Petersen, R. Neuendorf, K. AlShamery, and H.-G. Rubahn. Luminescence decay of oriented phenylene nanofibers. Phys. Rev. B, 72, 1152131-7, 2005.

[9] J. Brewer, M. Schiek, A. Lützen, K. Al-Shamery, and H.-G. Rubahn. Nanofiber frequency doublers. Nano Letters, 6, 2656-2659, 2006.

[10] F. Balzer, M. Schiek, A. Lützen, K. Al-Shamery, and H.-G.Rubahn. Growth of nanofibers from thiophenes, thiophene-phenylenes and phenylenes: a systematic study. Proc. SPIE, 6470, 2007. 
[11] D. Appell. Wired for success. Nature, 419, 554-555, 2002.

[12] M. Zacharias and P. Werner. Das Wachstum von Nanodrähten. Physik Journal, 4, 29-34, 2005.

[13] C. Ronning and D. Schwen. Nanodrähte, Nanosägen, Nanospiralen. PhiuZ, 37, 34-40, 2006.

[14] Y. Huang and C. Lieber. Integrated nanoscale electronics and optoelectronics: Exploring nanoscale science and technology through semiconductor nanowires. Pure Appl. Chem., 76, 2051-2068, 2004.

[15] C. Thelander, P. Agarwal, S. Brongersma, J. Eymery, L. Feiner, A. Forchel, M. Scheffler, W. Riess, B. Ohlsen, U. Gösle, and L. Samuelson. Nanowire-based one-dimensional electronics. Mat. Today, 9, 28-35, 2006.

[16] L. Tong, R.R. Gattass, J.B. Ashcom, S. He, J. Lou, M. Shen, I. Maxwell, and E. Mazur. Subwavelength-diameter silica wires for low-loss optical wave guiding. Nature, 426, 816-819, 2003.

[17] L. Tong, J. Lou, R.R. Gattass, S. He, X. Chen, L. Liu, and E. Mazur. Assembly of silica nanowires on silica aerogels for microphotonic devices. Nano Letters, 5, 259-262, 2005.

[18] Y. Huang, X. Duan, C.M. Lieber, and J.A. Schwarz (ed.). Semiconductor nanowires: Nanoscale electronics and optoelectronics. Dekker Encyclopedia of Nanoscience and Nanotechnology, Marcel Dekker, Inc., 1-32, 2005.

[19] Y. Cui, Q. Wei, H. Park, and C.M. Lieber. Nanowire nanosensors for highly sensitive and selective detection of biological and chemical species. Science, 293, 1289-1292, 2001.

[20] F. Patolsky and C.M. Lieber. Nanowire nanosensors. Mater. Today, April, 20-28, 2005.

[21] M.H. Huang, S. Mao, H. Feick, H. Yan, Y. Wu, H. Kind, E. Weber, R. Russo, and P. Yang. Room-temperature ultraviolet nanowire nanolasers. Science, 292, 1897-1899, 2001.

[22] Z.-M. Huang, Y.-Z. Zhang, M. Kotaki, and S. Ramakrishna. A review on polymer nanofibers by electrospinning and their applications in nanocomposites. Comp. Sci. Technol., 63, 2223-2253, 2003. 
[23] M. Bognitzki, W. Czado, T. Frese, A. Schaper, M. Hellwig, M. Steinhart, A. Greiner, and J.H. Wendorff. Nanostructured fibers via electrospinning. Adv. Mater., 13, 70$71,2001$.

[24] M. Steinhart, R.B. Wehrspon, U. Gösle, and J.H. Wendorff. Nanotubes by template wetting: A modular assembly system. Angew. Chem. Int. Ed., 43, 1334-1344, 2004.

[25] Sample preparation and imaging by SEM have been performed in the working group of Prof. Dr. J.H. Wendorff, University of Marburg. October, 2006.

[26] K. Müllen and G. Wegner. Electronic materials: The oligomer approach. WileyVCH, Weinheim, 1998.

[27] D. Fichou. Handbook of oligo- and polythiophenes. Wiley-VCH, Weinheim, 1999.

[28] H.S. Nalva. Handbook of advanced electronic and photonic materials and devices. Academic Press, San Diego, 2000.

[29] K. Müllen and U. Scherf. Organic light emitting devices - synthesis, properties and applications. Wiley-VCH, Weinheim, 2006.

[30] H. Klauk. Organic electronics - an industrial perspective. Wiley-VCH, Weinheim, 2006.

[31] P.F.H. Schwab, M.D. Levin, and J. Michl. Molecular rods. 1. simple axial rods. Chem. Rev., 99, 1863-1933, 1999.

[32] P.F.H. Schwab, J.R. Smith, and J. Michl. Synthesis and properties of molecular rods. 2. zig-zag rods. Chem. Rev., 105, 1197-1279, 2005.

[33] G. Ziegler and H.S. Nalwa (Ed.). Handbook of organic conductive molecules and polymers. Wiley, New York, Vol.3, chapter 13, 1997.

[34] B. Krause, A.C. Dürr, K. Ritley, F. Schreiber, H. Dosch, and D. Smilgies. Structure and growth morphology of an archetypal system for organic epitaxy: PTCDA on $\operatorname{Ag}(111)$. Phys. Rev. B, 66, 235404, 2002.

[35] F. Würthner. Perylene bisimide dyes as versatile building blocks for functional supramolecular architectures. Chem. Commun., 1564-1579, 2004.

[36] M. Bendikov, F. Wudl, and D.F. Perepichka. Tetrathiafulvalenes, oligoacenenes, and their buckminsterfullerene derivatives: The brick and mortar of organic electronics. Chem. Rev., 104, 4891-4945, 2004.

[37] D.K. James and J.M. Tour. Molecular wires. Top. Curr. Chem., 257, 33-62, 2005. 
[38] R. Resel. Crystallographic studies on hexaphenyl thin films - a review. Thin Solid Films, 433, 1-11, 2003.

[39] F. Balzer and H.-G. Rubahn. Growth control and optics of organic nanoaggregates. Adv. Funct. Mater., 14, 17-24, 2005.

[40] F. Balzer and H.-G. Rubahn. Optisch aktive Nanofasern: Selbstorganisierende organische Nanoaggregate. PhiuZ, 36, 36-43, 2005.

[41] G. Witte and C. Wöll. Growth of aromatic molecules on solid substrates for applications in organic electronics. J. Mater. Res., 19, 1889-1916, 2004.

[42] D. Hertel, C.D. Müller, and K. Meerholz. Organische Leuchtdioden: Bilderzeugung. ChiuZ, 39, 336-347, 2005.

[43] F. Balzer and H.-G. Rubahn. Dipole-assisted self-assembly of light-emitting p-np needles on mica. Appl. Phys. Lett., 79, 3860-3862, 2001.

[44] A. Andreev, G. Matt, C.J. Brabec, H. Sitter, D. Badt, H. Seyringer, and N.S. Sariciftci. Highly anisotropically self-assembled structures of para-sexiphenyl grown by hot-wall epitaxy. Adv. Mater., 12, 629-633, 2000.

[45] J. Stampfl, S. Tasch, G. Leising, and U. Scherf. Quantum efficiencies of electroluminescent poly(para)-phenylenes. Synth. Met., 71, 2125-2128, 1995.

[46] F. Balzer, V.G. Bordo, A.C. Simonsen, and H.-G. Rubahn. Isolated hexaphenyl nanofibers as optical waveguides. Appl. Phys. Lett., 82, 10-13, 2003.

[47] F. Balzer, V.G. Bordo, A.C. Simonsen, and H.-G. Rubahn. Optical waveguiding in individual nanometer-scale organic fibers. Phys. Rev. B., 67, 1154081-8, 2003.

[48] H. Yanagi and T. Morikawa. Self-waveguided blue light emission in p-sexiphenyl crystals epitaxially grown by mask-shadowing vapor deposition. Appl. Phys. Lett., 75, 187-189, 1999.

[49] F. Quochi, F. Cordella, R. Orru, J.E. Communal, P. Verzeroli, A. Mura, G. Bongiovanni, A. Andreev, H. Sitter, and N.S. Sariciftci. Random laser action in selforganized para-sexiphenyl nanofibers grown by hot-wall epitaxy. Appl. Phys. Lett., 84, 4454-4456, 2004.

[50] F. Quochi, F. Cordella, A. Mura, G. Bongiovanni, F. Balzer, and H.-G. Rubahn. One dimensional random lasing in a single organic nanofiber. J. Phys. Chem. B, 109, 21690-21693, 2005. 
[51] F. Quochi, F. Cordella, A. Mura, G. Bongiovanni, F. Balzer, and H.-G. Rubahn. Gain amplification and lasing properties of individual organic nanofibers. Appl. Phys. Lett., 88, 0411061-3, 2006.

[52] F. Quochi, A. Andreev, F. Cordella, R. Orru, A. Mura, G. Bongiovanni, H. Hoppe, H. Sitter, and N.S. Sariciftci. Low-threshold blue lasing in epitaxially grown parasexiphenyl nanofibers. J. Lumin., 112, 321-324, 2005.

[53] J. Kjelstrup-Hansen, H.H. Henrichsen, P. Boggild, and H.-G. Rubahn. Electrical properties of a single p-hexaphenylene nanofiber. Thin Solid Films, 515, 827-830, 2006.

[54] H.H. Henrichsen, J. Kjelstrup-Hansen, D. Engstroem, C.H. Clausen, P. Bogghild, and H.-G. Rubahn. Electrical conductivity of organic single-nanofiber devices with different contact materials. Appl. Phys. A, submitted, 2006.

[55] J. Kjelstrup-Hansen, P. Bogghild, and H.-G. Rubahn. Charge injection and transport in organic nanofibers. J. Phys. C, submitted, 2006.

[56] T. Mikami and H. Yanagi. Epitaxial structuring of blue light-emitting $p$-phenylene oligomers. Appl. Phys. Lett., 73, 563-565:563 - 565, 1998.

[57] H. Yanagi and T. Morikawa. Self-waveguided blue light emission in p-sexiphenyl crystals epitaxially grown by mask-shadowing vapor deposition. Appl. Phys. Lett., 75, 187-189, 1999.

[58] G. Koller, S. Berkebile, J.R. Krenn, G. Tzvetkov, G. Hlawacek, O. Lengyel, F.P. Netzer, C. Teichert, R. Resel, and M.G. Ramsey. Oriented sexiphenyl single crystal nanoneedles on $\mathrm{TiO}_{2}$ (110). Adv. Mater., 16, 2159-2162, 2004.

[59] T. Haber, A. Andreev, A. Thierry, H. Sitter, M. Oehzelt, and R. Resel. Parasexiphenyl thin films on $\mathrm{KCl}(100)$ surfaces: Growth morphologies and their individual epitaxial order. J. Cryst. Growth, 284, 209-220, 2005.

[60] C.B. France and B.A. Parkinson. Physical and electronic structure of $p$-sexiphenyl on Au(111). Appl. Phys. Lett., 82, 1194-1196, 2003.

[61] G. Koller, S. Surnev, M.G. Ramsey, and F.P. Netzer. Sexiphenyl on a Ni(110)(2×1)O surface: A single-molecule STM study. Surf. Sci., 559, L187-L193, 2004.

[62] S. Müllegger and A. Winkler. Hexaphenyl thin films on clean and carbon covered Au(111) studied with TDS and LEED. Surf. Sci., 600, 1290-1299, 2006. 
[63] Frank Balzer. Organic nanoaggregates. Habilitationsschrift, Humboldt-Universität zu Berlin, August, 2006.

[64] C. Ambrosch-Draxl, J.A. Majewski, P. Vogl, and G. Leising. First-principles studies of the structural and optical properties of crystalline poly(para-phenylene). Phys. Rev. B, 51, 9668-9676, 1995.

[65] H. Plank, R. Resel, S. Purger, J. Keckes, A. Thierry, B. Lotz, A. Andreev, N.S. Sariciftci, and H. Sitter. Heteroepitaxial growth of self-assembled highly ordered para-sexiphenyl films: A crystallographic study. Phys. Rev. B, 64, 235423, 2001.

[66] A.Y. Andreev, C. Teichert, G. Hlawacek, H. Hoppe, R. Resel, D.-M. Smilgies, H. Sitter, and N.S. Sariciftci. Morphology and growth kinetics of organic thin films deposited by hot wall epitaxy. Org. Electron., 5, 23- 27, 2004.

[67] F. Balzer. Growth of oriented organic nanoaggregates via organic molecular beam deposition. Springer Series in Material Science, Springer, Berlin, Organic Nanofibers for Next Generation Devices, K. Al-Shamery, H.-G. Rubahn, H. Sitter (eds.), 2007.

[68] F. Balzer and H.-G. Rubahn. Chain-length dependent para-phenylene film- and needle-growth on dielectrics. Surf. Sci., 548, 170-182, 2004.

[69] C. Teichert, G. Hlawacek, A.Y. Andreev, H. Sitter, P. Frank, A. Winkler, and N.S. Sariciftci. Spontaneous rearrangement of para-sexiphenyl crystallites into nanofibers. Appl. Phys. A, 82, 665-669, 2006.

[70] R.A. Knurr and S.W. Bailey. Refinement of Mn-substituted muscovite and phlogophite. Clays Clay Min., 34, 7-16, 1986.

[71] K. Müller and C.C. Chang. Electric dipoles on clean mica surfaces. Surf. Sci., 14, 39-51, 1969.

[72] K. Müller and C.C. Chang. Low energy electron diffraction observations of electric dipoles on mica surfaces. Surf. Sci., 9, 455-458, 1968.

[73] D.T. Griffen. Silicate crystal chemistry. Oxford University Press, New York, 1992.

[74] Y. Kuwahara. Muscovite surface structure imaged by fluid contact mode AFM. Phys. Chem. Minerals, 26, 198-205, 1999.

[75] W.E. Tröger. Optische Bestimmung der Gesteinsbildenden Minerale. Schweizerbartsche Verlagsbuchhandlung, Stuttgart, 4. Ausgabe, 1971.

[76] G. Tschermak. Lehrbuch der Mineralogie. Alfred Hölder, Wien, S. 135, 1894. 
[77] N. Uyeda, M. Ashida, and E. Suito. Orientation overgrowth of condensed polycyclic aromatic compounds vacuum-evaporated onto cleaved face of mica. J. Appl. Phys., 36, 1453-1460, 1965.

[78] M. Ashida. The orientation overgrowth of metal-phthalocyanines on the surface of single crystals. I. vacuum condensed films on muscovite. Bull. Chem. Soc. Jpn., 39, 2625-2631, 1966.

[79] F. Balzer, L. Kankate, H. Niehus, and H.-G. Rubahn. Nanoaggregates from oligothiophenes and oligophenylenes - a systematic survey. Proc. SPIE, 5724, 285-294, 2005.

[80] H. Yanagi, Y Araki, T. Ohara, S. Hotta, M. Ichikawa, and Y Taniguchi. Comparative carrier transport characteristics on organic field-effect transistors with vapordeposited thin films and epitaxially grown crystals of biphenyl-capped thiophene oligomers. Adv. Funct. Mater., 13, 767-773, 2003.

[81] M.-H. Yoon, A. Facchetti, C.E. Stern, and T.J. Marks. Fluorocarbon-modified organic semiconductors: Molecular architecture, electronic, and crystal tuning of arene- versus fluoroarene-thiophene oligomer thin-film properties. J. Am. Chem. Soc., 128, 5792-5801, 2006.

[82] A. Facchetti, M.-H. Yoon, C.L. Stern, H.E. Katz, and T.J. Marks. Building blocks for n-type organic electronics: Regiochemically modulated inversion of majority of carrier sign in perfluoroarene-modified polythiophene semiconductors. Angew. Chem. Int. Ed., 42, 3900-3903, 2003.

[83] M. Fanti and J. Almlöf. Hyperpolarizabilities of substituted polyphenyles. J. Molec. Struc. (Theochem), 388, 305-313, 1996.

[84] N. Matsuzawa and D.A. Dixon. Semiempirical calculations of hyperpolarizabilities for extended $\pi$-systems: polyenes, polyynes, and polyphenyls. Int. J. Cal. Quant. Chem., 44, 497-515, 1992.

[85] M. Schiek, T. Bruhn, K. Al-Shamery, R. Koch, A. Lützen, F. Balzer, J. Brewer, and H.-G. Rubahn. Tailored light emitting organic nanoaggregates generated by self-assembly of functionalised p-quaterphenylenes on muscovite mica substrates. Avd. Funct. Mater., in preparation, 2007.

[86] M. Albota, D. Beljonne, J.L. Bredas, J.E. Ehrlich, J.Y. Fu, A.A. Heikal, S.E. Hess, T. Kogej, M.D. Levin, S.R. Marder, D. McCord-Maughon, J.W. Perry, H. Roeckel, M. Rumi, G. Subramaniam, W.W. Web, X.L. Wu, and C. Xu. Design of organic molecules with large two-photon absorption cross sections. Science, 281, 1653, 1998. 
[87] L. Ventelon, S. Charier, L. Moreaux, J. Mertz, and M. Blanchard Desce. Nanoscale push-pull dihydrophenanthrene derivatives as novel fluorophores for two-photonexcited fluorescence. Angew. Chem. Int. Ed., 40, 2098-2101, 2001.

[88] V.R. Thalladi, S. Brasselet, H.-C. Weiss, D. Blaser, A.K. Katz, H.L. Carrell, R. Boese, J. Zyss, A. Nangia, and G.R. Desiraju. Octupolar nonlinear materials. J. Am. Chem. Soc., 120, 2563-2577, 1998.

[89] G.J. Ashwell. Langmuir-blodgett films: Molecular engineering of noncentrosymmetric structures for second order nonlinear optical applications. $J$. Mater. Chem., 9, 1991-2003, 1999.

[90] H. Schmidt and G. Schultz. Liebigs Ann., 203, 129, 1880.

[91] M.L. Scheinbaum. Nitration of p-quaterphenyl. J. Chem. Soc., Chem. Commun., 1235, 1969.

[92] T.G. Pavlopoulos and P.R. Hammond. Spectroscopic studies of some laser dyes. J. Am. Chem. Soc., 96, 6568-6579, 1974.

[93] M.A. Keegstra, S. De Feyter, F.C. De Schryver, and K. Müllen. Hexaterphenylyland hexaquaterphenylylbenzene: The behavior of chromophores and electrophores in a restricted space. Angew. Chem. Int. Ed., 35, 774-776, 1996.

[94] V.S. Iyer, M. Wehrmeier, J.D. Brand, M.A. Keegstra, and K. Müllen. From hexaperi-hexabenzocoronene to 'superacenes'. Angew. Chem. Int. Ed., 36, 1604-1607, 1997.

[95] M. Müller, V.S. Iyer, C. Kübel, V. Enkelmann, and K. Müllen. Polycyclic aromatic hydrocarbons by cyclodehydrogenation and skeletal rearrangement of oligophenylenes. Angew. Chem. Int. Ed., 36, 1607-1610, 1997.

[96] D. Fechtenköter, A. Saalwächter, M.A. Harbison, K. Müllen, and H.W. Spiess. Highly ordered columnar structures from hexa-peri-hexabenzocoronenes - synthesis, X-ray diffraction, and solid-state heteronuclear multiple-quantum NMR investigations. Angew. Chem. Int. Ed., 38, 3039-3042, 1999.

[97] S. Ito, P.T. Herwig, T. Böhme, J.P. Rabe, W. Rettig, and K. Müllen. Bishexaperi-hexabenzocoronenyl: A 'superbiphenyl'. J. Am. Chem. Soc., 122, 7698-7706, 2000 .

[98] J.K. Stille, R.O. Rakutis, H. Mukamal, and F. W. Harris. Diels-Alder polymerizations. IV. polymers containing short phenylene blocks connected by alkylene units. Macromolecules, 1, 431-436, 1968. 
[99] F. Morgenroth, E. Reuter, and K. Müllen. Polyphenylene dendrimers: From threedimensional to two-dimensional structures. Angew. Chem. Int. Ed., 36, 631-634, 1997.

[100] U.-M.Wiesler and K. Müllen. Polyphenylene dendrimers via Diels-Alder reactions: the convergent approach. Chem. Comm., 2293-2294, 1999.

[101] F. Dötz, J. D. Brand, S. Ito, L. Gherghel, and K. Müllen. Synthesis of large polycyclic aromatic hydrocarbons: Variation of size and periphery. J. Am. Chem. Soc., 122, 7707-7717, 2000.

[102] U.-M. Wiesler, A.J. Berresheim, F. Morgenroth, G. Lieser, and K. Müllen. Divergent synthesis of polyphenylene dendrimers: The role of core and branching reagents upon size and shape. Macromolecules, 34, 187-199, 2001.

[103] T. Weil, U.M. Wiesler, A. Herrmann, R. Bauer, J. Hofkens, F.C. De Schryver, and Klaus Müllen. Polyphenylene dendrimers with different fluorescent chromophores asymmetrically distributed at the periphery. J. Am. Chem. Soc., 123, 8101-8108, 2001.

[104] C.D. Simpson, J.D. Brand, A.J. Berresheim, L. Przybilla, H.J. Räder, and K. Müllen. Synthesis of a giant 222 carbon graphite sheet. Chem. Eur. J., 8, 1424-1429, 2002.

[105] G. Subramaniam and R.K. Gilpin. A convenient synthesis of 2',3"'"-dimethyl-psexiphenyl. Synthesis, 1232-1234, 1992.

[106] H. Hart and K. Harada. A new synthesis of para-terphenyls. Tetrahedron Lett., 26, 29-32, 1985.

[107] H. Hart, K. Harada, and C.-J. Frank Du. Synthetically useful aryl-aryl bond formation via Grignard generation and trapping of arynes. a one-step synthesis of p-terphenyl and unsymmetrical biaryls. J. Org. Chem., 50, 3104-3110, 1985.

[108] K. Harada, H. Hart, and C.-J. Frank Du. Reaction of aryl Grignard reagents with hexahalobenzenes: novel arenes via multiple aryne sequence. J. Org. Chem., 50, 5524-5528, 1985.

[109] C.-J. Frank Du, H. Hart, and K.-K. D. Ng. A one-pot synthesis of m-terphenyls, via a two-aryne sequence. J. Org. Chem., 51, 3162-3165, 1986.

[110] A. Rebmann, J. Zhou, P. Schuler, H.B. Stegmann, and A.J. Rieker. J. Chem. Res. (S), 318-319, 1996. 
[111] A. Rebmann, J. Zhou, P. Schuler, A.J. Rieker, and H.B. Stegmann. Synthesis of two novel para-extended bisaroxyls and characterization of their triplet spin states. J. Chem. Soc., Perkin Trans. 2, 1615-1617, 1997.

[112] J. Harley-Mason and F.G. Mann. J. Chem. Soc., 1379-1385, 1940.

[113] A. de Meijere and F. Diederich (ed.). Metal-catalyzed cross-coupling reactions. Wiley-VCH, Weihnheim, 2nd ed., 2004.

[114] J.J. Lie and G.W. Gribble. Palladium in herterocyclic chemistry. Pergamon Press, Elsevier, Amsterdam, 2000.

[115] N. Miyaura (ed.). Cross-coupling reactions. Springer, Berlin, Heidelberg, 2002.

[116] K. Tamao, T. Hiyama, and E. Negishi (ed.). Special issue on cross-coupling reactions. J. Organomet. Chem., 653, 1-299, 2002.

[117] H. Saitoh, K. Saito, Y. Yamamura, H. Matsuyama, K. Kikuchi, M. Iyoda, and I. Ikemoto. Crystal structures of the room-temperature phase of 4,4"-difluoro-pterphenyl and 4,4"'-difluoro-p-quaterphenyl. Bull. Chem. Soc. Jpn., 66, 2847-2853, 1993.

[118] V.A. Ung, D.A. Bardwell, J.C. Jeffery, J.P. Mahler, M.D. Ward, and A. Williamson. Dinuclear oxomolybdenum(V) complexes showing strong interactions across diphenol bridging ligands: Syntheses, structures, electrochemical properties, and EPR spectroscopic properties. Inorg. Chem., 35, 5290-5299, 1996.

[119] A. Abdul-Raman, A.A. Amoroso, T.N. Branston, A. Das, J.P. Mahler, J.A. McCleverty, M.D. Ward, and A. Wlodarczyk. Dinuclear molybdenum complexes derived from diphenols: electrochemical interactions and reduced species. Polyhedron, 16, 4353-4362, 1997.

[120] J.K. Kallitsis, F. Kakahli, and K.G. Gravalos. Synthesis and characterization of soluble aromatic polyesters containing oligophenyl moieties in the main chain. Macromolecules, 27, 4509-4515, 1994.

[121] J.K. Kallitsis, K.G. Gravalos, A. Hilberer, and G. Hadziioannou. Soluble polymers with laterally attached oligophenyl units for potential use as blue luminescent materials. Macromolecules, 30, 2989-2996, 1997.

[122] J.M. Kauffmann. A really convenient synthesis of 2',3"'-dimethyl-p-sexiphenyl. Synthesis, 918-920, 1999. 
[123] R. Rathore, C.L. Bruns, and M.I. Deselnicu. Multiple-electron transfer in a single step. design and synthesis of highly charged cation-radical salts. Org. Lett., 3, 28872890, 2001.

[124] P. Liess, V. Hensel, and A.-D. Schlüter. Liebigs Ann., 1037-1040, 1996.

[125] J. Frahn, B. Karakaya, A. Schäfer, and A.-D. Schlüter. Suzuki polycondensation: On catalyst derived phosphorus incorporation and reproducibility of molecular weights. Tetrahedron, 53, 15459-15467, 1997.

[126] V. Hensel and A.-D. Schlüter. A cyclotetraicosaphenylene. Chem. Eur. J., 5, 421429, 1999.

[127] N. Sakai, K.C. Brennan, L.A. Weiss, and S. Matile. Toward biomimetic ion channels formed by rigid-rod molecules: Length-dependent ion-transport activity of substituted oligo(p-phenylene)s. J. Am. Chem. Soc., 119, 8726-8727, 1997.

[128] B. Ghebremariam and S. Matile. Synthesis of asymmetric septi-(p-phenylene)s. Tetrahedron Lett., 39, 5335-5338, 1998.

[129] B. Ghebremariam, V. Sidorov, and S. Matile. Direct evidence for the importance of hydrophobic mismatch for cell membrane recognition. Tetrahedron Lett., 40, 1445-1448, 1999.

[130] J.-Y. Winum and S. Matile. Rigid push-pull oligo(p-phenylene) rods: Depolarization of bilayer membranes with negative membrane potential. J. Am. Chem. Soc., 121, 7961-7962, 1999.

[131] F. Robert, J.-Y. Winum, N. Sakai, D. Gerard, and S. Matile. Synthesis of multiply substituted, ion channel forming octi(p-phenylene)s: Theme and variations. Org. Lett., 2, 37-39, 2000 .

[132] N. Sakai, D. Gerard, and S. Matile. Electrostatics of cell membrane recognition: Structure and activity of neutral and cationic rigid push-pull rods in isoelectric, anionic, and polarized lipid bilayer membranes. J. Am. Chem. Soc., 123, 25172524, 2001.

[133] N. Sakai and S. Matile. Recognition of polarized lipid bilayers by p-oligophenyl ion channels: From push-pull rods to push-pull barrels. J. Am. Chem. Soc., 124, 1184-1185, 2002.

[134] P. Galda and M. Rehahn. A versatile palladium-catalyzed synthesis of n-alkylsubstituted oligo-p-phenyls. Synthesis, 614-620, 1996. 
[135] S. Kim, J. Jackiw, E. Robinson, K.S. Schanze, and J.R. Reynolds. Water soluble photo- and electroluminescent alkoxy-sulfonated poly(p-phenylenes) synthesized via palladium catalysis. Macromolecules, 31, 964-974, 1998.

[136] M.B. Goldfinger, K.B. Crawford, and T.M. Swager. Synthesis of ethynyl-substituted quinquephenyls and conversion to extended fused-ring structures. J. Org. Chem., 63, 1676-1686, 1998.

[137] F.D. Konstandakopoulou, K.G. Gravalos, and J.K Kallitsis. Synthesis and characterization of processable aromatic-aliphatic polyethers with quinquephenyl segments in the main chain for light-emitting applications. Macromolecules, 31, 5264-5271, 1998.

[138] A. Morikawa. Preparation and properties of hyperbranched poly(ether ketones) with a various number of phenylene units. Macromolecules, 31, 5999-6009, 1998.

[139] B. Schlicke, P. Belser, L. De Cola, E. Sabbioni, and V. Balzani. Photonic wires of nanometric dimensions. electronic energy transfer in rigid rodlike $\mathrm{Ru}(\mathrm{bpy})_{3}{ }^{2+}$ (ph)n-Os(bpy) ${ }_{3}^{2+}$ compounds $(\mathrm{ph}=1,4$-phenylene; $\mathrm{n}=3,5,7)$. J. Am. Chem. Soc., 121, 4207-4214, 1999.

[140] P.N. Taylor, M.J. O'Connell, L.A. McNeill, M.J. Hall, R.T. Alpin, and H.L. Anserson. Insulated molecular wires: Synthesis of conjugated polyrotaxanes by Suzuki coupling in water. Angew. Chem. Int. Ed., 39, 3456-3460, 2000.

[141] M.W. Read, J.O. Escobedo, D.M. Willis, P.A. Beck, and R.M. Strongin. Convenient iterative synthesis of an octameric tetracarboxylate-functionalized oligophenylene rod with divergent end groups. Org. Lett., 2, 3201-3204, 2000.

[142] S.-W. Hwang and Y. Chen. Synthesis and electrochemical and optical properties of novel poly(aryl ether)s with isolated carbazole and p-quaterphenyl chromophores. Macromolecules, 34, 2981-2986, 2001.

[143] J.-W. Park, M.D. Ediger, and M.M. Green. Chiral studies in amorphous solids: The effect of the polymeric glassy state on the racemization kinetics of bridged paddled binaphthyls. J. Am. Chem. Soc., 123, 49-56, 2001.

[144] X. Deng, A. Mayeux, and C. Cai. An efficient convergent synthesis of novel anisotropic adsorbates based on nanometer-sized and tripod-shaped oligophenylenes end-capped with triallylsilyl groups. J. Org. Chem., 67, 5279-5283, 2002.

[145] S. Lightowler and M. Hird. Palladium-catalyzed cross-coupling reactions in the synthesis of novel aromatic polymers. Chem. Mater., 16, 3963-3971, 2004. 
[146] S. Lightowler and M. Hird. Monodisperse aromatic oligomers of defined structure and large size through selective and sequential suzuki palladium-catalyzed crosscoupling reactions. Chem. Mater., 27, 5538-5549, 2005.

[147] V. Percec and S. Okita. Synthesis and Ni(0)-catalyzed oligomerization of isomeric 4,4' '-dichloroquaterphenyls. J. Polymer Sci., Part A, 31, 877-884, 1993.

[148] Z.H. Li, M.S. Wong, Y. Tao, and M. D Iorio. Synthesis and functional properties of strongly luminescent diphenylamino end-capped oligophenylenes. J. Org. Chem., 69, 921-927, 2004.

[149] M. Lee, C.-J. Jang, and J.-H. Ryu. Supramolecular reactor from self-assembly of rod-coil molecule in aqueous environment. J. Am. Chem. Soc., 126, 8082-8083, 2004.

[150] J.-H. Ryu, C.J. Jang, Y.-S. Yoo, S.-G. Lim, and M. Lee. Supramolecular reactor in an aqueous environment: Aromatic cross suzuki coupling reaction at room temperature. J. Org. Chem., 70, 8956-8962, 2005.

[151] S. Welter, N. Salluce, A. Benetti, N. Rot, P. Belser, P. Sonar, A.C. Grimsdale, K. Müllen, M. Lutz, A.L. Spek, and L. de Cola. Rodlike bimetallic ruthenium and osmium complexes bridged by phenylene spacers. synthesis, electrochemistry, and photophysics. Inorg. Chem., 44, 4706-4718, 2005.

[152] K.N. Baker, A.V. Fratini, T. Resch, and H.C. Knachel. Crystal structures, phase transitions and energy calculations of poly(p-phenylene) oligomers. Polymer, 34, 1571-1587, 1993.

[153] Markus Jordan. Synthese von symmetrisch und unsymmetrisch substituierten Oligophenylenethinylenen. Diploma Thesis, University of Oldenburg, Sept., 2005.

[154] M. Schwoerer and H.C. Wolf. Organische Molekulare Festkörper - Einführung in die Physik von $\pi$-Systemen. Wiley-VCH, Weinheim, 2005.

[155] M.D. Blanchard, R.P. Hughes, T.E. Concolino, and A.L. Rheingold. $\pi$-stacking between pentafluorophenyl and phenyl groups as a controlling feature of intra- and intermolecular crystal structure motifs in substituted ferrocenes. observation of unexpected facte-to-face stacking between pentafluorophenyl rings. Chem. Mater., 12, 1604-1610, 2000.

[156] K.N. Baker, A.V. Fratini, T. Resch, H.C. Knachel, W.W. Adams, E.P. Socci, and B.L. Farmer. Crystal structures, phase transition and energy calculations of polyp-phenylene oligomers. Polymer, 34, 1571-1587, 1993. 
[157] H. Saitoh, K. Saito, Y. Yamamura, H. Matsuyama, K. Kikuchi, and I. Ikemoto. X-ray study on structural phase transitions of 4,4"-difluoro-p-terphenyl and 4,4"'difluoro-p-quaterphenyl. Sol. Stat. Comm., 91, 89-92, 1994.

[158] M. Moret. private communication, June 2006.

[159] F. Balzer and H.-G. Rubahn. Laser-controlled growth of needle-shaped organic nanoaggregates. Nano Letters, 2, 747-750, 2002.

[160] A. Niko, F. Meghdadi, C. Ambrosch-Draxel, P. Vogel, and G. Leising. Optical absorbance of oriented thin films. Synth. Met., 76, 177-179, 1996.

[161] P. Puschnig and C. Ambrosch-Draxel. Density-functional study of the oligomers of poly-para-phenylene: Band structures and dielectric tensors. Phys. Rev. B, 60, 7891-7898, 1999.

[162] P.A. Campbell, L.J. Sinnamon, C.E. Thompson, and D.G. Walmsley. Atomic force microscopy evidence of $\mathrm{K}^{+}$domains on freshly cleaved mica. Surf. Sci., 410, L768L772, 1998.

[163] K. Hermann and M.A. van Hove. LEEDpat 2.0, 2002.

[164] F. Biscarini, R. Zamboni, P. Samori, P. Ostoja, and C. Taliani. Growth of conjugated oligomer thin films studied by atomic-force microscopy. Phys. Rev. B, 52, 14868-14877, 1995.

[165] J.A. Venables, G.D.T. Spiller, and M. Hanbucken. Nucleation and growth of thin films. Rep. Prog. Phys., 47, 399-459, 1984.

[166] H. Plank, R. Resel, H. Sitter, A. Andreev, N.S. Sariciftci, G. Hlawacek, C. Teichert, A. Thierry, and B. Lotz. Molecular alignment in sexiphenyl thin films epitaxially grown on muscovite. Thin Solid Films, 443, 108-114, 2003.

[167] M. Ohring. Materials science of thin films. Academic Press, San Diego, 1992.

[168] F. Würthner, S. Ahmed, C. Thalacker, and T. Debaerdemaeker. Core-substituted naphthalene bisimides: New fluorophores with tunable emission wavelength for FRET studies. Chem. Eur. J., 8, 4742-4750, 2002.

[169] A. Blaszcyk, M. Fischer, C. v.Hänisch, and M. Mayor. Synthesis, structure, and optical properties of terminally sulfur-functionalized core-substituted naphthalenebisimide dyes. Helv. Chim. Acta, 89, 1986-2005, 2006.

[170] B. Valeur. Molecular fluorescence: Principles and applications. Wiley VCH, 2001. 
[171] H.K. Christenson. Adhesion and surface energy of mica in air and water. J. Phys. Chem., 97, 12034-12041, 1993.

[172] F. Balzer, J. Beermann, S.I. Bozhevolnyi, A.C. Simonsen, and H.-G. Rubahn. Optically active organic microrings. Nano Letters, 3, 1311-1314, 2003.

[173] G. Koller, S. Berkebile, J.R. Krenn, F.P. Netzer, M. Oehzelt, T. Haber, R. Resel, and M.G. Ramsey. Heteroepitaxy of organic-organic nanostructures. Nano Letters, 6, $1207-1212,2006$.

[174] C.E. Mortimer. Chemie - Das Basiswissen der Chemie. Thieme Stuttgart, New York, 6. Aufl., 1996.

[175] P. Pringsheim and H. Kronenberger. Z. Physik, 40, 75, 1926.

[176] J. Frenkel. On the transformation of light into heat in solids. II. Phys. Rev., 37, 1276-1294, 1931.

[177] A.S. Davydov. J. Exp. Theor. Phys. USSR (engl. transl.), 7, 145, 1964.

[178] F.C. Spano. Excitons in conjugated oligomer aggregates, films, and crystals. Ann. Rev. Phys. Chem., 57, 217-243, 2006.

[179] E. Zojer, N. Koch, P. Puschnig, F. Meghdadi, A. Niko, R. Resel, C. AmbroschDraxl, M. Knupfer, J. Fink, J.L. Bredas, and G. Leising. Structure, morphology, and optical properties of highly ordered films of para-sexiphenyl. Phys. Rev. B, 61, 16538-16549, 2000.

[180] E. Zojer, M. Knupfert, Z. Shuai, J.L. Bredas, J. Fink, and G. Leising. The quasiband-structure description of conjugated oligomers. J. Phys.: Condens. Matter, 12, 1753-1768:1753 - 1768, 2000.

[181] S. Hotta, Y. Ichino, Y. Yoshida, and M. Yoshida. Spectroscopic features of thin films of thiophene/phenylene co-oligomers with vertical molecular alignment. $J$. Phys. Chem. B, 104, 10316-10320, 2000.

[182] E.A. Meyer, R.K. Castellano, and F. Diederich. Interactions with aromatic rings in chemical and biological recognition. Angew. Chem. Int. Ed., 42, 1210-1250, 2003.

[183] M. Hesse, H. Meier, and B. Zeeh. Spektroskopische Methoden in der organischen Chemie. Thieme, Stuttgart, New York, 5. Aufl., 1995.

[184] J. Brewer, H.H. Henrichsen, F. Balzer, L. Bagatolli, A.C. Simonsen, and H.-G. Rubahn. Nanofibers made to order: Free floating, transferred and gel-packed organic nanoaggregates. Proc. SPIE, 5931, 250-257, 2005. 
[185] J. Brewer, C. Maibohm, L. Jozefowski, L. Bagatolli, and H.-G. Rubahn. A 3D view on free-floating, space-fixed and surface-bound para-phenylene nanofibers. Nanotechnology, 16, 2396-2401, 2005.

[186] Y.R. Shen. The principles of nonlinear optics. Wiley, New York, 1984.

[187] F. Balzer, K. Al-Shamery, R. Neuendorf, and H.-G. Rubahn. Nonlinear optics of hexaphenyl nanofibers. Chem. Phys. Lett., 368, 307-312, 2003.

[188] Jonathan R. Brewer. PhD Thesis, University of Southern Denmark, in preparation, 2007.

[189] J. Brewer and H.-G. Rubahn. Optical frequency doubling and imaging of functionalized organic nanofibers. Opt. Lett., in preperation, 2007.

[190] G. Polanski and H.-G. Rubahn. Excimer laser assisted growth of Au thin films on mica (001). J. Vac. Sci. Technol. A, 14, 110-114, 1996.

[191] G. Binnig, C.F. Quate, and C. Gerber. Atomic force microscope. Phys. Rev. Lett., 56, 930-933, 1986.

[192] F.J. Giesibl. AFMs path to atomic resolution. MaterialsToday, May, 32-41, 2005.

[193] JPK Instruments. The NanoWizard AFM Handbook. Version 1.3, 2005.

[194] W.A. Herrmann. Synthetic methods of organometallic and inorganic chemistry. Thieme Verlag, Stuttgart, 7-9, 75, 1997.

[195] V.A. Ung, D.A. Bardwell, J.C. Jeffery, J.P. Maher, J.A. McCleverty, M.D. Ward, and A. Williamson. Dinuclear oxomolybdenum(V) complexes showing strong interactions across diphenol bridging ligands: Syntheses, structures, electrochemical properties, and EPR spectroscopic properties. Inorg. Chem., 35, 5290-5299, 1996.

[196] A. Abdul-Rahman, A.A. Amoroso, T.N. Branston, A. Das, J.P. Maher, J.A. McCleverty, M.D. Ward, and A. Wlodaczyk. Dinuclear molybdenum complexes derived from diphenols: electrochemical interactions and reduced species. Polyhedron, 16, 4353-4362, 1997.

[197] A. Ronlan, J. Coleman, and V.D. Parker O. Hammerich. Anodic oxidation of methoxybiphenyls. effect of the biphenyl linkage on aromatic cation radical and dication stability. J. Am. Chem. Soc., 96, 845-849, 1974.

[198] V. Percec and S. Okita. Synthesis and Ni(0)-catalyzed oligomerization of isomeric 4,4 ' 'dichloroquaterphenyls. J. Polymer Sci., Part A, 31, 877-884, 1993. 
[199] A. Jutand and A. Mosleh. Palladium and nickel catalyzed synthesis of biaryls from aryl triflates in the presence of zinc powder. Synlett, 568-570, 1993.

[200] A. Jutand and A. Mosleh. Nickel- and palladium-catalyzed homocoupling of aryl triflates. scope, limitation, and mechanistic aspects. J Org. Chem., 62, 261-274, 1997.

[201] W. Theilacker, W. Berger, and P. Popper. The formation of this compound has been postulated as an undesired byproduct in the synthesis of triarylmethane dyes, however, no analytical or structural proof was given. Chem. Ber., 89, 970-983, 1956.

[202] M.L. Schienbaum. J. Chem. Soc., Chem. Commun., 1235, 1969.

[203] K. Biradha, Y. Hongo, and M Fujita. Open square-grid coordination polymers of the dimensions 20 x 20 å: Remarkably stable and crystalline solids even after guest removal. Angew. Chem. Int. Ed., 39, 3843-3845, 2000.

[204] W.W. Porter, T.P. Vaid, and A.L. Rheingold. Synthesis and characterization of highly reducing neutral "extended viologen" and the isostructural hydrocarbon 4,4"'”-di-n-octyl-p-quaterphenyl. J. Am. Chem. Soc., 127, 16559-16566, 2005.

[205] C.J. Kelly, K. Ansu, W. Budisusetyo, A. Ghiorghis, Y. Qin, and J.M. Kauffman. Synthesis and photophysical properties of some 4-arylpyridinium salts. J. Hetrocyclic Chem., 38, 11-23, 2001.

[206] F. Thiemann, T. Piehler, D. Haase, W. Saak, and A. Lützen. Synthesis of enantiomerically pure dissymmetric 2,2'-disubstituted-9,9'-spirobifluorenes. Eur. J. Org. Chem., 10, 1991-2001, 2005.

[207] J.A. Ragan, T.W. Makowski, M.J. Castaldi, and P.D. Hill. 2,5-dimethylpyrrole protection facilitates copper(I)-mediated methoxylations of aryl iodides in the presence of anilines. Synthesis, 1599-1603, 1998.

[208] P.E. Broutin, I. Cerna, M. Campaniello, F. Leroux, and F. Colobert. Palladiumcatalyzed borylation of phenyl bromides and application in one-pot suzuki-miyaura biphenyl synthesis. Org. Lett., 6, 4419-4422, 2004.

[209] M. Murata, T. Oyama, S. Watanabe, and Y. Mazuda. Palladium-catalyzed borylation of aryl halides or triflates with dialkoxyborane: A novel and facile synthetic route to arylboronates. J. Org. Chem., 65, 164-168, 2000.

[210] C. Coudret. Efficient synthesis of 4-iodpyridine and of 4-pyridylboronic acid pinacol ester. Synth. Comm., 26, 3543-3547, 1996. 
[211] Y. Han, S.D. Walker, and R.N. Young. Silicon directed ipso-substitution of polymer bound arylsilanes: Preparation of biaryls via the suzuki cross-coupling reaction. Tetrahedron Lett., 37, 2703-2706, 1996.

[212] A. C. Spivey, C.M. Diaper, and A.J. Rudge. A new germanium based linker for solid phase synthesis of aromatic compounds. Chem. Commun., 835-836, 1999.

[213] A.C. Spivey, C.M. Diaper, H. Adams, and A.J. Rudge. A new germanium-based linker for solid phase synthesis of aromatics: Synthesis of a pyrazole library. J. Org. Chem., 65, 5253-5263, 2000.

[214] D.J. Sinclair and M.S. Sherburn. Single and double suzuki-miyaura couplings with symmetric dihalobenzenes. J. Org. Chem., 70, 3730-3733, 2005.

[215] R. Pummerer and L Sellsberger. Chem. Ber., 64, 2477-2486, 1931.

[216] J.M. McNamara and W.B. Gleason. Synthesis of 4-cyano-4'-halobiphenyls. J. Org. Chem., 41, 1071, 1976.

[217] W. Theilacker and W. Schmid. Chem. Ber., 84, 204-210, 1951.

[218] W. Theilacker, W. Berger, and P. Popper. Chem. Ber., 89, 970-983, 1956.

[219] A.M. Sedov, A.A. Sergeeva, and A.N. Novikov. Izvestija Vysshikh Uchebnykh Zavedenii, Khimiya I Khimicheskaya Tekhnologiya, 13, 591-592, CAN73:87559, 1970.

[220] M. Rottaelander, N. Palmer, and P. Knochel. Selective $\operatorname{Pd}(0)$-catalyzed arylations with new electrophilic or nucleophilic multi-coupling reagents. Synlett, 573-575, 1996.

[221] C. Amatore, A. Jutand, S. Negere, and J.F. Fauvarque. Efficient palladiumcatalyzed synthesis of unsymmetrical donor - acceptor biaryls and polyaryls. $J$. Organomet. Chem., 390, 389-398, 1990.

[222] A. N. Novikov and T.A. Khalimova. Zhurnal Vsesoyuznogo Khimicheskogo Obshchestva, 7, 698, CAN 58:66196, 1962.

[223] S. Hotta, H. Kimura, S.A. Lee, and T. Tamaki. Synthesis of thiophene/phenylene co-oligomers II block and alternating co-oligomers. J. Heterocyclic. Chem., 37, 281$286,2000$.

[224] A. Pelter and D.E. Jones. The preparation and some properties of phenyleneethynylene and phenylene-1,3-diynylene polymers. J. Chem. Soc., Perkin Trans. 1, 2289-2294, 2000. 
[225] L. Liu, Z. Liu, W. Xu, H. Xu, D. Zhang, and D. Zhu. Syntheses, optical and electrochemical properties of 4,4'-bis-[2-(3,4-dibutyl-2-thienylethynyl)] biphenyl and its oligomers. Tetrahedron, 61, 3813-3817, 2005.

[226] W. Steinkopf, R. Leitsmann, and K.H. Hofman. Justus Liebigs Ann. Chem., 546, 180, 1941.

[227] J.W. Sease and L. Zechmeister. Chromatographic and spectral characteristics of some polythienyls. J. Am. Chem. Soc., 69, 270-273, 1947.

[228] G. Horowitz, D. Fichou, X.Z. Peng, and Z.G. Xu. A field-effect transistor based on conjugated $\alpha$-sexithienyl. Sol. Stat. Commun., 72, 381-384, 1989.

[229] F. Geiger, M. Stoldt, H. Schweizer, P. Bauerle, and E. Umbach. Electroluminescence from oligothiophene-based light-emitting devices. Adv. Mater., 5, 922-925, 1993.

[230] K. Uchiyama, H. Achimichi, S. Hotta, H. Noge, and H. Sakaki. Electroluminescence from thin film of a semiconducting oligothiophene. Synth. Met., 63, 57, 1994.

[231] J. Roncali. Conjugated poly(thiophenes): synthesis, functionalization, and applications. Chem. Rev., 92, 711-738, 1992.

[232] S. Hotta and S.A. Lee. Various chemical modifications of oligothienyls and oligophenyls. Synth. Met., 101, 551-552, 1999.

[233] T.J. Dingemans, A. Bacher, M. Thelakkat, L.G. Pedersen, E.T. Samulski, and H.W. Schmidt. Spectral tuning of light emitting diodes with phenyl-thiophenes. Synth. Met., 105, 171-177, 1999.

[234] H. Tian, J. Shi, D. Yang, L. Wang, Y. Geng, and F. Wang. Naphthyl end-capped quarterthiophene: A simple organic semiconductor with high mobility and air stability. Adv. Mater., 18, 2149-2152, 2006.

[235] H. Wang, J. Wang, X. Yan, J. Shi, H. Tian, Y. Geng, and D. Yan. Ambipolar organic field-effect transistor with air stability, high mobility, and balanced transport. Appl. Phys. Lett., 88, 133508, 2006.

[236] S. Mohapatra, B.T. Holmes, C.R. Newman, C.F. Prendergast, C.D. Frisbie, and M.D. Ward. Organic thin-film transistors based on tolyl-substituted oligothiophenes. Adv. Funct. Mater., 14, 605-609, 2004.

[237] H. Yanagi, T. Morikawa, and S. Hotta. Electroluminescence from low-dimensionally confined crystals of thiophene/phenylene co-oligomers. Appl. Phys. Lett., 81, 15121514, 2002. 
[238] J. Casado, M.C. Ruiz Delgado, Y. Shirota, V. Hernandez, and J.T. Lopez Navarrete. Computation and spectroelectrochemistry as complementary tools for the study of electrochemically induced charged defects in 4- bis(4-methylphenyl)amino-phenyl oligothiophenes as model systems for hole-transporting materials. J. Phys. Chem. B, 107, 2637-2644, 2003.

[239] M. Ichikawa, H. Yanagi, Y. Shimizu, S. Hotta, N. Suganuma, T. Koyama, and Y. Taniguchi. Organic field-effect transistors made of epitaxially grown crystals of a thiophene/phenylene co-oligomer. Adv. Mater., 14, 1272-1275, 2002.

[240] S.A. Lee, Y. Yoshida, M Fukuyama, and S. Hotta. Phenyl-capped oligothiophenes: novel light emitting materials with different molecular alignments in thin films. Synth. Met., 106, 39-43, 1999.

[241] H. Yanagi, T. Morikawa, S. Hotta, and K. Yase. Epitaxial growth of thiophene/pphenylene co-oligomers for highly polarized light-emitting crystals. Adv. Mater., 13, 313-317, 2001.

[242] Y. Yoshida, N. Tanigaki, K. Yase, and S. Hotta. Color-tunable highly polarized emissiom from unaxially aligned thin films of thiophene/phenylene co-oligomers. Adv. Mater., 12, 1587-1591, 2000.

[243] C. Videlot-Ackermann, J. Ackermann, H. Brisset, K. Kawamura, N. Yoshimoto, P. Raynal, A. El Kassmi, and F. Fages. $\alpha, \omega$-distyryl oligothiophenes: High mobility semiconductors for environmentally stable organic thin film transistors. J. Am. Chem. Soc., 127, 16346-16347, 2005.

[244] F. Sasaki, S. Haraich, S. Kobayashi, and S. Hotta. Spectrally narrowed emission of thiophene/phenalene co-oligomer films deposited on $\mathrm{Si}$ and $\mathrm{SiO}_{2}$ substrates. Proc. Int. Symp. Super-Functionality Organic Devices, IPAP Conf. Series 6, 161-163.

[245] H. Yanagi, A. Yoshiki, S. Hotta, and S. Kobayashi. Stimulated resonance raman scattering from single crystals of a thiophene/phenylene co-oligomer. Appl. Phys. Lett., 83, 1941-1943, 2003.

[246] K. Shimizu, D. Hoshino, and S. Hotta. Unusually narrowed emission lines from a single crystal of thiophene/phenylene co-oligomer. Appl. Phys. Lett., 83, 4494-4496, 2003.

[247] K. Bando, T. Nakamuru, Y Masumoto, F. Sasaki, S Kobayashi, and S. Hotta. Origin of the amplified spontaneous emission from thiophene/phenylene co-oligomer single crystals: Towards co-oligomer lasers. J. Appl. Phys., 99, 013518, 2006. 
[248] M. Nagawa, R. Hibino, S. Hotta, H. Yanagi, M. Ichikawa, T. Koyama, and Y. Taniguchi. Emission gain narrowing from single crystals of a thiophene/phenylene co-oligomer. Appl. Phys. Lett., 80, 544-546, 2002.

[249] M. Ichikawa, R. Hibino, M. Inoue, T. Haritani, S. Hotta, T. Koyama, and Y. Taniguchi. Improved crystal-growth and emission gain-narrowing of thiophene/phenylene co-oligomers. Adv. Mater., 15, 213-217, 2003.

[250] H. Yanagi, A. Yoshiki, S. Hotta, and S. Kobayashi. Mirrorless lasing from thiophene/phenylene co-oligomer crystals based on stimulated resonance Raman scattering. J. Appl. Phys., 96, 4240-4244, 2004.

[251] M. Ichekawa, R. Hibino, M. Inoue, T. Haritani, S. Hotta, K. Araki, T. Koyama, and Y. Taniguchi. Laser oscillation on monolithic molecular single crystals. Adv. Mater., 17, 2073-2077, 2005.

[252] S. Hotta and T. Katagiri. Synthesis of thiophene/phenylene co-oligomeres IV 6- to 8-ring molecules. J. Heterocyclic. Chem., 40, 845-850, 2003.

[253] S. Hotta, S.A. Lee, and T. Tamaki. J. Heterocyclic Chem., 37, 25, 2000.

[254] S. Hotta. J. Heterocyclic Chem., 38, 923, 2001.

[255] K. Tamao, S. Kodama, I. Nakajima, M. Kumada, A. Minato, and K. Suzuki. Nickelphosphine complex-catalyzed Grignard coupling II Grignard coupling of heterocyclic compounds. Tetrahedron, 38, 3347-3354, 1982.

[256] T. Mitsuhara, K. Kaeriyama, and S. Tanaka. New electrochromic polymers. J. Chem. Soc., Chem. Commun., 10, 764-765, 1987.

[257] S. A. Lee, S. Hotta, and F. Nakanishi. Spectroscopic characteristics and intermolecular interactions of thiophene/phenylene co-oligomers in solutions. J. Phys. Chem. A, 104, 1827-1833, 2000.

[258] S. Hotta, M. Goto, R. Azumi, M. Inoue, M. Ichikawa, and Y. Taniguchi. Crystal structures of thiophene/phenylene co-oligomers with different molecular shapes. Chem. Mater., 16, 237-241, 2004.

[259] F. Balzer, L. Kankate, H. Niehus, R. Frese, Ch. Maibohm, and H.-G. Rubahn. Tayloring the growth of p-6P nanofibres by ultrathin Au layers: An organic - metal - dielectric model system. Nanotechnology, 17, 984-991, 2006. 
Bibliography 


\section{Acknowledgements}

First I have to thank my doctoral advisor Prof. Dr. Katharina Al-Shamery for giving me the possibility to do a thesis with this challenging conceptual formulation and allowing me great latitude to tap the full potential.

I am indebted to Prof. Dr. Arne Lützen and Prof. Dr. Peter Köll for providing excellent working conditions concerning Organic Chemistry, and to Prof. Dr. Arne Lützen for continuous supervision and helpful enthusiasm for physics of nanofibers.

I am deeply grateful to Prof. Dr. Horst-Günter Rubahn for providing excellent working conditions concerning Physics and Optics, and for continuous supervision including giving me the feeling to be always welcome.

I appreciate my german chemistry colleagues Torsten Piehler, Jens Bunzen, Oliver Hass, Ulf Kiehne, Holger Staats, Susanne Heinze, Lars Brandt, Alexander Schierholdt and Eike Harlos for just being nice colleagues and sharing coffe in the morning and beer in the evening, and especially Markus Jordan and Ivonne Wallmann for additional interest in nanofibers and providing molecules for those. Particularly I thank Ivonne for the really promising molecules and team work during her visit in Odense.

I thank my danish (or so) physics colleagues Morten Madsen, Jonathan Brewer, Kasper Thilsing-Hansen, Ralf Frese and further Christian Maibohm, Henrik Henrichsen, Jacek Fiutowski, Thomasz Kawalec and Anna Machjer for good collaboration in a friendly and relaxed atmosphere and being nice colleagues, especially I thank Jonathan for sharing results concerning the non linear optics. And further on I thank Danielle Keller, Esben Thormann, Stefan Vogel and Katharina Rubahn for some fun and me just being welcome.

Some further indirect colleagues I thank for amusing chats Alexander Winkler, Robert Buchwald, Ulrich Leist, Kai Schröder, Anna Plader, Daniela Fenske, Mandus Necke, Dirk Hogestraat, Ewa Bozek-Winkler, Andreas Rausch, Eileen Collinet, Knut Schulz, Lüder Diedrich, Juliane Walter, Ingmar Piglosiewicz, Mira Diekmann, Radek Masur and maybe more.

I thank Richard Dawton for commenting on my English.

Some exclusive private thanks go to:

- my parents, Agathe and Reinhard Schiek for private and financial support generously all the time.

- Silke Nebig for being a real friend all the time and looking after mice, plants and stuff I left in Oldenburg during my stays in Odense. 
- Charlotte, Lars, Sybil and Johannes Yde, the dogs Karla and Darcy for giving my always the feeling to be welcome and of being kind of at home in Odense, and further my flatmates Stanco and Yordanka.

- 'my' horse Pikeur and people from the riding stable Helga, Steffi, Nico, Erika, Maren, Kurt, Vera, Gabi, Ursel and few more, and further on other friends or so ;-) such as Michael Döker, Thomas Loewenstein, Mario Steinker and more for making my private life valuable during my $\mathrm{PhD}$-time in Oldenburg.

Last but definitively not least I gratefully thank Dr. Frank Balzer, who has maybe the closest relation to this thesis, supporting me generously in practice and theory making lot more possible, answering thousands of emails, and having some nice time together beyond work. 


\section{Curriculum Vitae}

$\begin{array}{ll}\text { name } & \text { Manuela Schiek } \\ \text { private address } & \text { Franz-Poppe-Str. 15, D-26121 Oldenburg, Germany } \\ \text { working address } & \text { University of Oldenburg, } \\ & \text { IRAC, P.O.-Box 2503, D-26111 Oldenburg, Germany } \\ \text { email } & \text { manuela.schiek@uni-oldenburg.de } \\ \text { born } & \text { 08-08-1978 in Wilhelmshaven, Germany } \\ \text { family status } & \text { unmarried, no children } \\ \text { nationality } & \text { german }\end{array}$

\section{Education}

from May 2007 Postdoctoral stay in Sonderborg at University of Southern Denmark in NANOLASE research group of Prof. Dr. H.-G. Rubahn.

May 2004 Doing PhD under supervision of

until Prof. Dr. K. Al-Shamery (Oldenburg, Physical Chemistry),

Feb. 2007 Prof. Dr. A. Lützen (Oldenburg, Organic Chemistry), and Prof. Dr. H.-G. Rubahn (Odense, Denmark, Physics).

June-Sept. 2006 Research stay in Odense at the University of Southern Denmark

and in research group of Prof. Dr. H.-G. Rubahn

June-Aug. 2005 in context of PhD.

May $2006 \quad$ Week-long research stay in Marburg, Germany, in research group of Prof. Dr. J. Wendorff.

April 2004 Diploma in Chemistry at the University of Oldenburg, Germany under supervision of Prof. Dr. K. Al-Shamery.

Nov. $2000 \quad$ Pre-Diploma in Chemistry at the University of Oldenburg.

Okt. 1998 Begin of study of Chemistry at the University of Oldenburg.

July $1998 \quad$ School leaving exam Abitur

at the Gymnasium am Mühlenweg in Wilhelmshaven, Germany.

Okt. 1995 Guest stay at the J.B. Conant High School in Chicago, Illinois.

1985-1991 Elementary schools in Wilhelmshaven, Germany.

languages German (mother tongue), English (fluently written and spoken), Latin (Magnum Latinum), Old Greek (Graecum). 


\section{Publications}

- M. Schiek, A. Lützen, R. Koch, K. Al-Shamery, F. Balzer, R. Frese, and H.-G. Rubahn, Nanofibers from functionalized para-phenylene molecules, Appl. Phys. Lett., 86, 153107-9, 2005.

- M. Schiek, A. Lützen, K. Al-Shamery, F. Balzer, and H.-G. Rubahn, Nanofibers from methoxy functionalized para-phenylene molecules, Surf. Sci., 600, 4030-4033, 2006.

- J. Brewer, M. Schiek, A. Lützen, K. Al-Shamery, and H.-G. Rubahn, TITLEPAGE: Nanofiber frequency doublers, Nano Letters, 6, 2656-2659, 2006.

- M. Schiek, K. Al-Shamery, M. Kunat, F. Traeger, and Ch. Wöll, D 2 O-Adsorption at a $\mathrm{H}(1 \times 1)-\mathrm{O}-\mathrm{ZnO}(000 \overline{1})$ surface, Phys. Chem. Chem. Phys., 8, $1505-1512$, 2006.

- M. Schiek, K. Al-Shamery, and A. Lützen, Synthesis of symmetrically and nonsymmetrically para-functionalised para-quaterphenylenes, Synthesis, 4, 613 - 621, 2007.

- M. Schiek, A. Lützen, K. Al-Shamery, F. Balzer, and H.-G. Rubahn, Organic nanofibers from chloride functionalized para-quaterphenylenes, Cryst. Growth \&6 Design, 7, 229 - 233, 2007.

- M. Schiek, J. Brewer, F. Balzer, A. Lützen, K. Al-Shamery, and H.-G. Rubahn, Tailored nanoaggregates from functionalized organic molecules, Proc. SPIE, 6475$17,2007$.

- F. Balzer, M. Schiek, A. Lützen, K. Al-Shamery, and H.-G. Rubahn, Growth of nanofibers from thiophenes, thiophene-phenylenes and phenylenes: a systematic study, Proc. SPIE, 6470-06, 2007.

- K. Nonomura, T. Loewenstein, E. Michaelis, P. Kunze, M. Schiek, J. Reemts, M. Iwaya, M. Wark, J. Rathousky, K. Al-Shamery, A. Kittel, J. Parisi, D. Wöhrle, T. Yoshida D. Schlettwein, Nanoparticulate dye-semiconductor hybrid materials formed by electrochemical self-assembly as electrodes in photoelectrichemical cells, Small, submitted 2006.

- K. Al-Shamery, M. Schiek, R. Koch, A. Lützen, Tailored organic nanoaggregates generated by self-assembly of designed functionalised p-quaterphenylenes on muscovite mica substrates, in Springer Series in Material Science, Springer, Berlin, Organic Nanofibers for Next Generation Devices, K. Al-Shamery, H.-G. Rubahn, H. Sitter (eds.) in preparation 2007. 
- M. Schiek et al., B. Pignataro (Ed.), Organic Molecular Nanotechnology: Light Emitting Organic Nanoaggregates from functionalised para-Quaterphenylenes, in Highlights of Chemistry - An Organic and Materials Science View, Wiley-VCH, Weinheim, in preparation 2007.

\section{Patent Applications}

- M. Schiek, A. Lützen, K. Al-Shamery, R. Koch, F. Balzer, H.-G. Rubahn, Nanofibers from functionalized Organic Molecules, European Patent Application, University of Oldenburg, University of Southern Denmark, submitted April 2006.

\section{Talks}

- M. Schiek, J. Brewer, K. Al-Shamery, A. Lützen, F. Balzer, H.-G. Rubahn, Tailored nanoaggregates from functionalized organic molecules, SPIE Photonic West, Integrated Optics: Devices, Materials, and Technologies XI, San Jose, California, USA, 20.1. to 25.1.2007.

- M. Schiek, A. Lützen, K. Al-Shamery, F. Balzer, H.-G. Rubahn, Light emitting nanofibers from functionalised para-quaterphenylenes, 1st European Chemistry Congress, Budapest, Ungary, 27.8. to 31.8.2006.

- M. Schiek, Nanofibers from functionalized para-Phenylene Molecules, Meeting of research groups: Albrecht (Aachen), Lützen (Bonn), Schalley (Berlin), Bonn, Germany, 20.7. to 22.7.2006.

- M. Schiek, A. Lützen, K. Al-Shamery, F. Balzer, H.-G. Rubahn, Nanofibers from functionalized para-Phenylene Molecules, 23. European Conference on Surface Science in Berlin, Germany, 7.9.2005.

- M. Schiek, A. Lützen, R. Koch, K. Al-Shamery, F. Balzer, R. Frese, H.-G. Rubahn, Funktionalisierte para-Phenylen Nanonadeln, 104. Bunsentagung, Frankfurt, Germany, 7.5.2005.

- M. Schiek, Organic Nanofibers from para-Phenylenes, Meeting of research groups: Albrecht (Aachen), Lützen (Bonn), Schalley (Berlin), Oldenburg, Germany, 14.2.2005.

\section{Posters}

- M. Schiek, A. Lützen, K. Al-Shamery, F. Balzer, H.-G. Rubahn, Light emitting nanofibers from functionalised para-quaterphenylenes, CAMS / Jobs, SDU, Sönder- 
borg, Denmark, 11.10. to 12.10. 2006.

- M. Schiek, A. Lützen, K. Al-Shamery, F. Balzer, H.-G. Rubahn, Light emitting nanofibers from functionalised para-quaterphenylenes, 1st European Chemistry Congress, Budapest, Ungary, 27.8. to 31.8.2006.

- F. Balzer, L. Kankate, H. Niehus, M. Schiek, A. Lützen, K. Al-Shamery, H.-G. Rubahn, Nanofibers from thiophene oligomeres, 1st European Chemistry Congress, Budapest, Ungary, 27.8. to 31.8.2006.

- J. Brewer, M. Schiek, H.-G. Rubahn, Nanofiber frequency doublers, 3rd Nanoworkshop, SDU, Odense, Denmark, 12.6.2006.

- M. Schiek, K. Al-Shamery, Water at clean and silver modified $H(1 \times 1)-O-Z n O(0001)$, 105. Bunsentagung, Erlangen, Germany, 26.5. to 27.5.2006.

- R. Koch, M. Schiek, K. Al-Shamery, A. Lützen, H.-G. Rubahn, Light emitting nanofibers from functionalised para-quaterphenylenes, Nanotechnology in Northern Europe, Helsinki, Finland 16.5.2006. Winning Poster of Best Poster Award.

- M. Schiek, A. Lützen, R. Koch, K. Al-Shamery, F. Balzer, H.-G. Rubahn, Leuchtende organische Nanofasern, Tag der Chemie, University of Oldenburg, Germany, 26.1.2006.

- M. Schiek, K. Al-Shamery, Water at clean and silver modified H(1×1)-O-ZnO(0 $00 \overline{1})$, International Workshop on Nanostructured Semiconductor Electrodes, Gießen, Germany, 19.10. to 21.10.2005.

- M. Schiek, K. Al-Shamery, Water at clean and silver modified $H(1 \times 1)-O-Z n O(0001)$, 89th International Bunsen Discussion Meeting, Meschede, Germany, 15.6. to 17.6.2005.

- M. Schiek, A. Lützen, R. Koch, K. Al-Shamery, F. Balzer, R. Frese, H.-G. Rubahn, Organic Nanofibers from functionalised para-Phenylenes, Workshop on 2- and 3dimensional Organic Nanostructure, Linz, Austria, 9.6. to 11.6. 2005.

\section{Grants}

- Support from the SPIE student travel contingency grant for attendance of SPIE Photonic West, San Jose, California, USA, January 2007.

- Winning poster of Best Poster Award at Nanotechnology in Northern Europe, Helsinki, Finland, May 2006. 
- GDCh travel grant for attendance of 1st European Chemistry Congress in Budapest, Ungary, August 2006.

- Nominated for Degussa Nano\&Art Price, February 2006.

- Congress Scholarship of the Oldenburger bank manager awarded by the UGO (Universitätsgesellschaft Oldenburg) for attendance of ECOSS 23, Berlin, Germany, September 2005. 